\title{
Inertial Fusion Energy Target Injection, Tracking, and Beam Pointing
}

R. W. Petzoldt

(Ph.D. Thesis)

March 7, 1995 


\section{DISCLAIMER}

This document was prepared as an account of work sponsored by an agency of the United States Government. Neither the United States Government nor the University of California nor any of their employees, makes any warranty, express or implied, or assumes any legal liability or responsibility for the accuracy, completeness, or usefulness of any information, apparatus, product, or process disclosed, or represents that its use would not infringe privately owned rights. Reference herein to any specific commercial product, process, or service by trade name, trademark, manufacturer, or otherwise, does not necessarily constitute or imply its endorsement, recommendation, or favoring by the United States Government or the University of California. The views and opinions of authors expressed herein do not necessarily state or reflect those of the United States Government or the University of California, and shall not be used for advertising or product endorsement purposes.

This report has been reproduced directly from the best available copy.

Available to DOE and DOE contractors from the

Office of Scientific and Technical Information

P.O. Box 62, Oak Ridge, TN 37831

Prices available from (615) 576-8401, FTS 626-8401

Available to the public from the

National Technical Information Service

U.S. Department of Commerce

5285 Port Royal Rd.

Springfield, VA 22161

Work performed under the auspices of the U.S. Department of Energy by Lawrence Livermore National Laboratory under Contract W-7405-ENG-48. 


\section{DISCLAIMER}

Portions of this document may be illegible in electronic image products. Images are produced from the best available original document. 


\section{Inertial Fusion Energy Target Injection, Tracking, and Beam Pointing}

R. W. Petzoldt

(Ph.D. Thesis)

Manuscript date: March 7, 1995

LAWRENCE LIVERMORE NATIONAL LABORATORY

University of California • Livermore, California • 94551 


\section{Inertial Fusion Energy}

Target Injection, Tracking, and Beam Pointing

\section{By}

Ronald Wayne Petzoldt

B.A. (Saint Cloud State University, Saint Cloud, MN) 1981

M.S. (University of California, Davis) 1991

\section{DISSERTATION}

Submitted in partial satisfaction of the requirements for the degree of

DOCTOR OF PHILOSOPHY

in

Applied Science

in the

GRADUATE DIVISION

of the

UNIVERSITY OF CALIFORNIA

DAVIS

Approved:

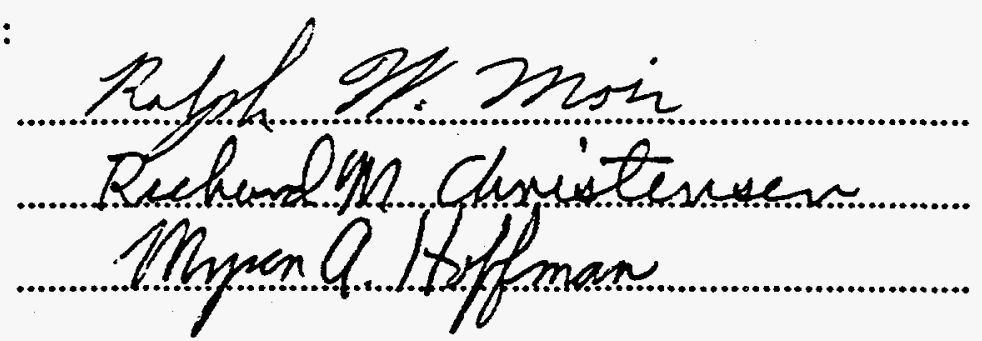

Committee in Charge

1995 


\section{Inertial Fusion Energy \\ Target Injection, Tracking, and Beam Pointing}

\section{Abstract}

Several cryogenic targets must be injected each second into a reaction chamber. Required target speed is about $100 \mathrm{~m} / \mathrm{s}$ using acceleration in the range of 1,000 to $10,000 \mathrm{~m} / \mathrm{s}^{2}$. Required accuracy of the driver beams on target is plus or minus a few hundred micrometers.

Fuel strength is calculated to allow acceleration in excess of $10,000 \mathrm{~m} / \mathrm{s}^{2}$ if the fuel temperature is less than $17 \mathrm{~K}$. A $0.1 \mu \mathrm{m}$ thick dual membrane will allow nearly $2,000 \mathrm{~m} / \mathrm{s}^{2}$ acceleration. Acceleration is gradually increased and decreased over a few membrane oscillation periods (a few $\mathrm{ms}$ ), to avoid added stress from vibrations which could otherwise cause a factor of two decrease in allowed acceleration.

Movable shielding allows multiple targets to be in flight toward the reaction chamber at once while minimizing neutron heating of subsequent targets. The use of multiple injectors is recommended for redundancy which increases availability and allows a higher pulse rate.

Gas gun, rail gun, induction accelerator, and electrostatic accelerator target injection devices are studied and compared. A gas gun is the preferred device for indirect-drive targets due to its simplicity and proven reliability. With the gas gun, the amount of gas required for each target (about 10 to $100 \mathrm{mg}$ ) is acceptable. A revolver loading mechanism is recommended with a cam operated poppet valve to control the gas flow. Cutting vents near the muzzle of the gas gun barrel is recommended to improve accuracy and aid gas 
pumping. If a railgun is used, we recommend an externally applied magnetic field to reduce required current by an order of magnitude.

Optical target tracking is recommended. Up/down counters (electronic timing devices which function independent of target speed) are suggested to predict target arrival time. Target steering is shown to be feasible and would avoid the need to actively point the beams. Calculations show that induced tumble from electrostatically steering the target is not excessive. The total required accuracy of target injection, tracking and beam pointing of $\pm 0.4 \mathrm{~mm}$ appears achievable but will require development and experimental verification. 


\section{Acknowledgements}

I would especially like to thank Dr. Ralph W. Moir who served as my research advisor and mentor throughout my time at UC and LLNL. He always made himself available to discuss and review my work. He provided countless helpful suggestions and recommendations. I also would like to thank Dr. Richard M. Christensen who graciously agreed to chair my thesis committee when Dr. Roger A. Haas stepped down from that position. Dr. Christensen also provided many useful suggestions, particularly in the area of continuum mechanics. Dr. Myron Hoffman provided many helpful suggestions for the improvement of the draft dissertation.

Many others from Lawrence Livermore National Laboratory provided input to parts of this work especially Palmer House who suggested the revolver loading mechanism, Andy Anderson who tutored me in the use of NIKE2D, and Robert Horton who suggested the use of up/down counter to time target arrival.

This work was performed under the auspices of the U. S. Department of Energy by Lawrence Livermore National Laboratory under Contract W7405-Eng-48. 


\section{Table of Contents}

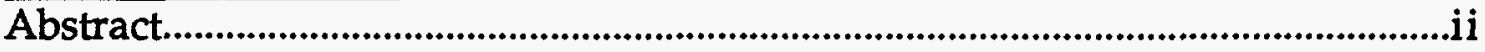

Acknowledgements........................................................................................................

Table of Contents .............................................................................................................

List of Figures...........................................................................................................vii

Chapter 1- Introduction .................................................................................................1

1.1. Fusion Energy .................................................................................................1

1.2. An IFE Power Plant ...................................................................................4

1.3. References....................................................................................................

Chapter 2 - Target Injection, Tracking, and Beam Pointing

Requirements and Feasibility Considerations....................................................9

2.1. Introduction ............................................................................................

2.2. Acceleration, Speed, Injection Distance, and Rate

Relationships

2.2.1. Basic Parameters......................................................................12

2.2.2. Implications of Assuming Target Acceleration Time is Less than Interpulse Time.

2.2.3. Implications of Using Multiple Injectors ...............................20

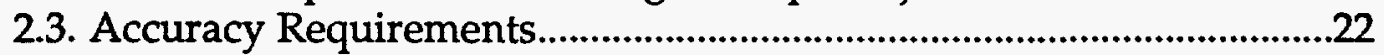

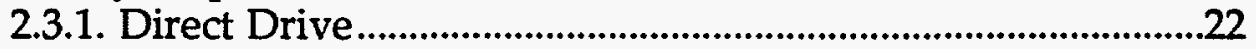

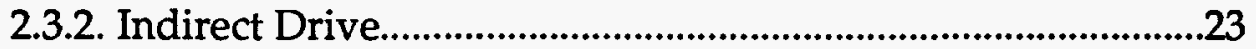

2.3.3. Gyroscopic Target Stabilization...............................................27

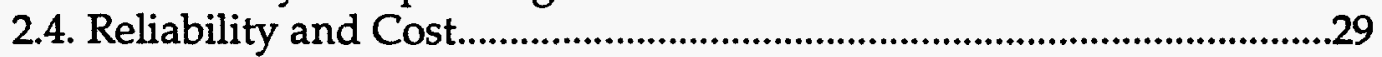

2.5. Target Shielding.........................................................................................32

2.5.1. Movable Shielding ......................................................................32

2.5.2. Target Trajectory Shielding ………………..................................37

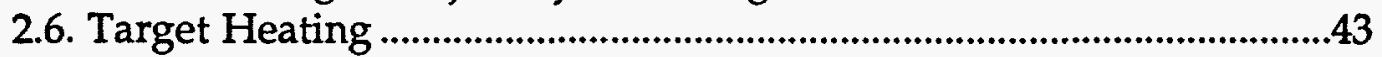

2.6.1. DT Fuel Temperature Limits .................................................43

2.6.2. Conduction Heat-transfer During the Injection

Process . .47

2.6.3. Convective Heat-transfer During the Injection Process .56

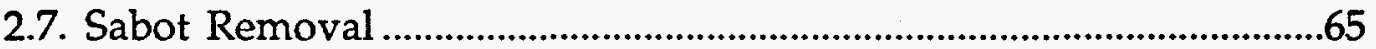

2.8. Summary of Injection Requirements ...................................................73

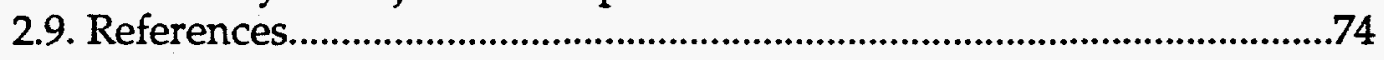

Chapter 3 - Membrane Support of Accelerated Fuel Capsules.............................78

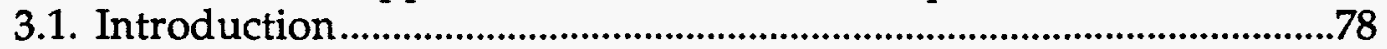

3.2. Effects of Membrane on Illumination Symmetry .................................80

3.3. Membrane Problem Description .............................................................81

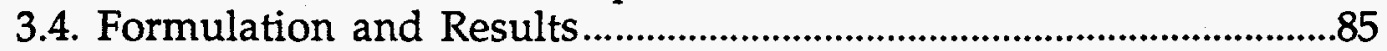

3.4.1. Linear Formulation.................................................................85

3.4.2. Nonlinear Formulation ............................................................91

3.5. Effects of Membrane Vibration........................................................109

3.5.1. Vibration Frequency................................................................110 
3.5.2. Vibration Amplitude and Resulting Position

Uncertainty

3.6. References.

4.1. Problem Definition and Solution Method...........................................117

4.2. Computational Results.............................................................................119

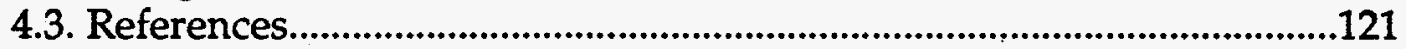

Chapter 5 - Target Steering.........................................................................................122

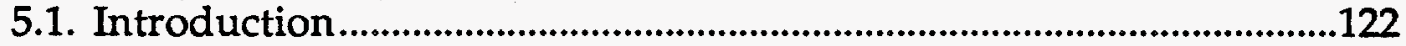

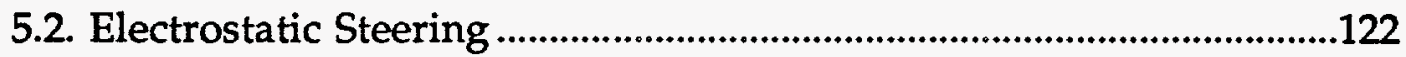

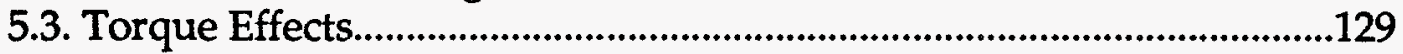

5.4. Computer Simulation .........................................................................130

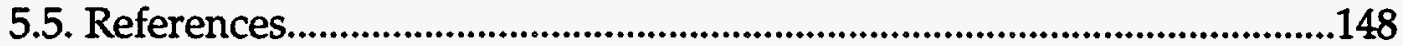

Chapter 6 - Target Injection Methods.....................................................................149

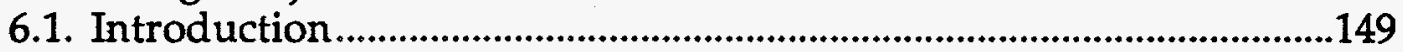

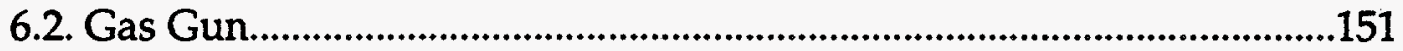

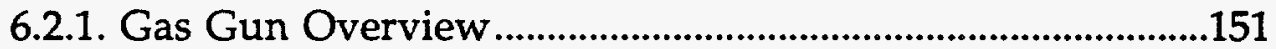

6.2.2. Gas Flow Control and Pumping.............................................153

6.2.2.1. Cam and Poppet Valve Flow Control ....................153

6.2.2.2. Vacuum Pumping Design ...........................................155

6.2.2.3. Gun Barrel Venting..................................................157

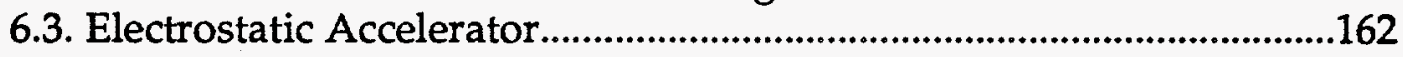

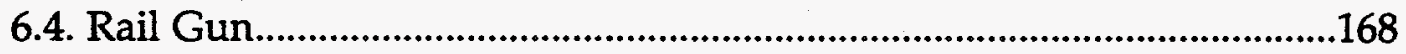

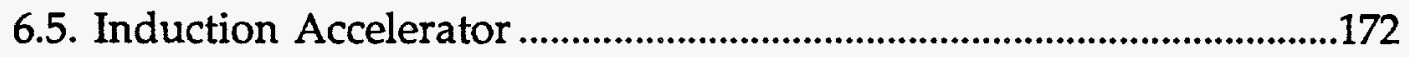

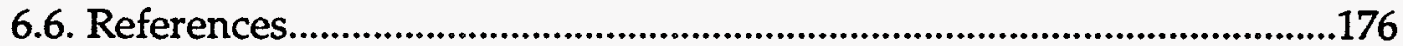

Chapter 7 - Target Tracking and Beam Pointing .....................................................179

7.1. Target Tracking.........................................................................................179

7.1.1. Photodiode Timing Detectors ....................................................179

7.1.2. Detector Shielding .....................................................................179

7.1.3. Comparator Circuits................................................................182

7.1.4. Up/down Counters ..............................................................183

7.1.5. Transverse Position Sensing..................................................186

7.1.6. Target Tracking and Beam Pointing Drift ..............................188

7.2. Beam Pointing .............................................................................................188

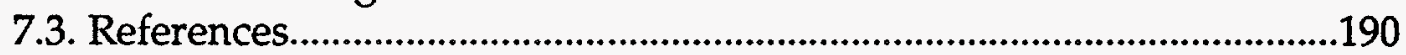

Chapter 8 - Summary, Conclusions, and Recommendations.............................192

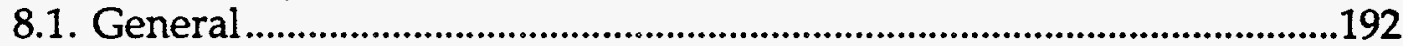

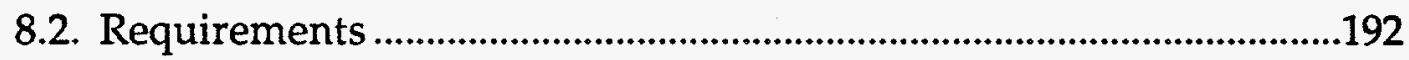

8.3. Structural Limits on Acceleration.........................................................194

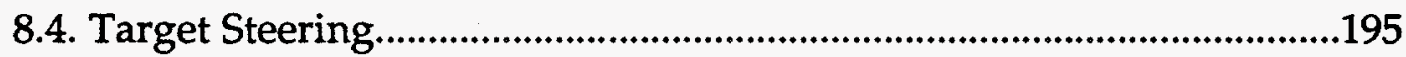

8.5. Target Injection Methods....................................................................195

8.6. Target Tracking and Beam Pointing...........................................................196

8.7. Recommendations for Future Work ...............................................196

8.7.1. Proposed Target Injection and Tracking Experiment .........197

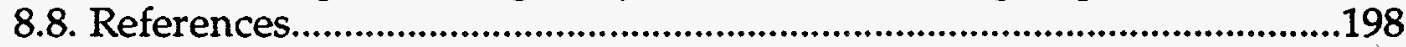

Selected Bibliography .....................................................................................................199 


\section{List of Figures}

Fig. 1-1 Direct-drive capsule........................................................................................2

Fig. 1-2 Heavy ion indirect-drive target ..................................................................3

Fig. 1-3 HYLIFE-II reaction chamber ....................................................................5

Fig. 1-4 Artist's rendering of HYLIFE-II power plant ............................................6

Fig. 1-5 IFE reaction chamber with target injector and final part of driver beams 7

Fig. 2-1 Target acceleration illustration ...........................................................12

Fig. 2-2 Speed and acceleration limits for target injection ....................................14

Fig. 2-3 Target speed vs. acceleration for various acceleration lengths..............16

Fig. 2-4 Target speed vs. acceleration design considerations ..............................18

Fig. 2-5 Multiple target injectors........................................................................22

Fig. 2-6a Target positioning limitations (two-sided illumination target)........24

Fig. 2-6b Target positioning limitations (one-sided illumination target)........26

Fig. 2-7 Indirect drive target rotated off axis ............................................................27

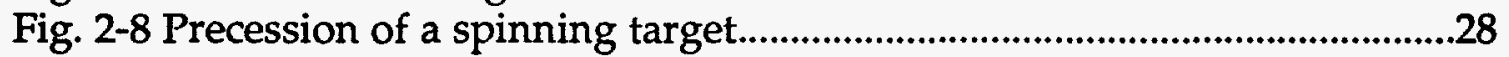

Fig. 2-9 Movable target neutron shield .....................................................................33

Fig. 2-10 Schematic of target trajectory shielding......................................................39

Fig. 2-11 Graph of target trajectory shielding ..........................................................40

Fig. 2-12 Schematic of target trajectory shielding with finite hole......................41

Fig. 2-13 Inertial fusion energy target .........................................................................44

Fig. 2-14 Model used to calculate target heating.....................................................48

Fig. 2-15a Indirect-drive target temperature vs time.............................................50

Fig. 2-15b Indirect-drive target fuel temperatures vs time....................................51

Fig. 2-16 Direct-drive target temperature vs time.....................................................54

Fig. 2-17 Direct-drive target outer DT temperature vs time for various

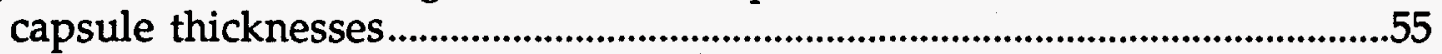

Fig. 2-18 Direct-drive target outer DT temperature vs time for various

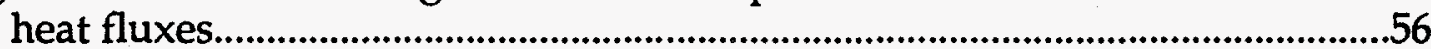

Fig. 2-19 Convection heat-transfer flow paths ......................................................58

Fig. 2-20a Target temperature vs time with convective heating.........................60

Fig. 2-20b Fuel temperature vs time with convective heating .............................61

Fig. 2-21 Sabot separation from capsule (3D)..........................................................65

Fig. 2-22 Geometry of two piece and four piece sabots........................................66

Fig. 2-23 Relationship between prime and unprime coordinate systems .......68

Fig. 2-24a Path that the upper half of a two piece of the sabot would take

if not restrained by the capsule ............................................................................71

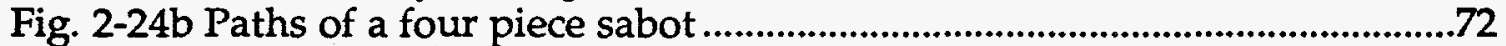

Fig. 3-1 Targets with single and dual membrane support.......................................79

Fig. 3-2 Dual membrane axisymmetric target configuration................................80

Fig. 3-3 3D membrane deformation geometry..........................................................82

Fig. 3-4 Stress vs strain for mylar at various temperatures ....................................84

Fig. 3-5 Geometry and forces applicable to an axisymmetric membrane..........86

Fig. 3-6 Geometry of a conical membrane ...........................................................90 
Fig. 3-7 Normalized capsule displacement vs normalized capsule

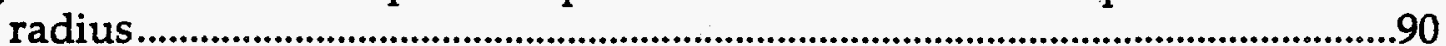

Fig. 3-8 A differential element of the deformed membrane ...............................92

Fig. 3-9 Differential cross-section of membrane....................................................101

Fig. 3-10 Membrane angle vs membrane strain....................................................103

Fig. 3-11 Membrane displacement vs membrane strain ........................................105

Fig. 3-12 Comparison of single and dual membrane for achieving

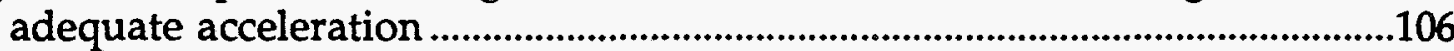

Fig. 3-13 Rounded hohlraum/membrane interface................................................109

Fig. 3-14 Hohlraum acceleration and capsule displacement vs time................113

Fig. 3-15 Maximum capsule displacement vs acceleration rise time..................114

Fig. 3-16 Residual capsule oscillation ............................................................115

Fig. 4-1 An accelerated fuel capsule.........................................................................118

Fig. 4-2 Stresses in fuel and membrane for an accelerated fuel capsule............120

Fig. 5-1 Schematic of target steering in electric field................................................123

Fig. 5-2 Maximum potential difference and electrode field vs. electrode spacing.

Fig. 5-3 Maximum surface potential and electric field of a negatively charged sphere vs. radius. 126

Fig. 5-4 Maximum negative charge on sphere vs. radius ..................................127

Fig. 5-5 Charge separation causing torque in an electric field ..............................130

Fig. 5-6 Geometry of target for torque calculations.................................................131

Fig. 5-7 Finite difference grid for torque calculations.............................................131

Fig. 5-8 Electric field around target between steering electrodes...........................132

Fig. 5-9 Simulated box in electric field...................................................................134

Fig. 5-10 Cathode electric field for steering electrode (target not charged).......139

Fig. 5-11 Torque on target between steering electrodes (target not

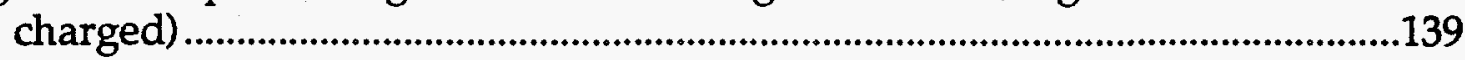

Fig. 5-12 Cathode electric field for steering electrode (target charged)..............140

Fig. 5-13 Torque on target between steering electrodes (target charged) ...........140

Fig. 5-14 Target charge between steering electrodes................................................141

Fig. 5-15 Force on target between steering electrodes............................................141

Fig. 5-16 Force on target between steering electrodes (maximum field)...........142

Fig. 5-17 Torque on target between steering electrodes (maximum field).......142

Fig. 5-18 Moment of inertia vs. target shape..........................................................143

Fig. 6-1 Inertial fusion energy reaction chamber with target injector and

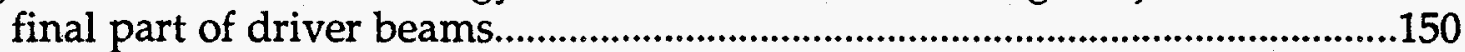

Fig. 6-2 Gas gun target injector with vents for gas to escape..............................152

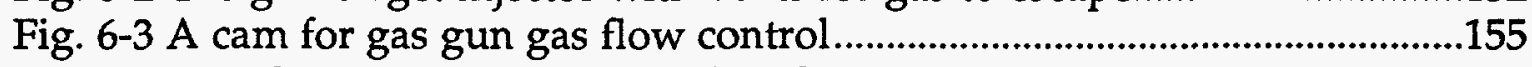

Fig. 6-4 Barrel gas pressure vs. position during venting........................................160

Fig. 6-5 Electrostatic accelerator ..................................................................................165

Fig. 6-6 Target charging and insertion method for electrostatic acceleration. 168

Fig. 6-7 Schematic of solid armature rail gun .....................................................171

Fig. 6-8 Simplified model to estimate the currents and fields associated with a railgun target injector. 
Fig. 6-9 A 6 phase induction accelerator.

.172

Fig. 7-1 Target position detection with photodiodes

Fig. 7-2 A comparator circuit aids precise target timing .183

Fig. 7-3 An analog up/down counter 185

Fig. 7-4 A digital up/down counter. .186

Fig. 7-5 Crossed photodiode detectors to measure target lateral position 187

Fig. 7-6 A capacitive detector to measure target position 188

Fig. 7-7 Ion beam steering with magnetic field .189 


\section{Chapter 1- Introduction}

\subsection{Fusion Energy}

The fusion of light nuclei to produce heavier nuclei is the primary source of energy in our universe. Fusion produces the energy that powers the sun and stars. To undergo fusion, the nuclei in a plasma must overcome the electrostatic repulsion between them. A temperature of order $100,000,000 \mathrm{~K}$ $(10 \mathrm{keV})$ is normally required so that the nuclei will have sufficient kinetic energy to overcome the potential energy barrier between them. These high temperature nuclei must be held near each other for sufficient time for fusion reactions to occur. In the stars, gravitational force confines a relatively low temperature plasma for very long time periods. An equilibrium is set up such that as the fusion reaction rate increases, the plasma temperature rises, the plasma becomes less dense, effectively decreasing the fusion reaction rate.

On earth, two processes, magnetic confinement and inertial confinement, have been successful in confining fusion plasmas. The nuclei that have the highest probability for fusing at the lowest temperature are deuterium and tritium. The reaction they undergo is

$$
{ }_{1}^{2} \mathrm{H}+{ }_{1}^{3} \mathrm{H} \rightarrow{ }_{2}^{4} \mathrm{He}+{ }_{0}^{1} n+17.6 \mathrm{MeV} \text {. }
$$

The product of the density times the time for these reactions to produce energy gain is ideally about $10^{14} \mathrm{~cm}^{-3} \mathrm{~s}$ and more realistically about $10^{15} \mathrm{~cm}^{-}$ ${ }^{3}$ s. ${ }^{1}$ Magnetic confinement uses magnetic fields to confine low density plasmas for seconds. Inertial confinement fusion uses inertial effects to confine very high density plasmas for about 30 picoseconds. $^{2}$ 
For inertial confinement, a high density, high temperature plasma is achieved as follows. DT is frozen to the inner surface of a low atomic number spherical capsule as indicated in Fig. 1-1. There will also be DT gas in the capsule in equilibrium with the DT frozen to the inner surface of the capsule. Driver energy, such as laser beams or ion beams, heat the capsule surface. The surface ablates and the resulting gas expands. As a reaction to the gas expansion, the fuel is compressed in toward the center. A central hot spot develops in which fusion reactions occur. If the product of the compressed fuel density times the radius is sufficiently large, the alpha particles produced in the hot spot fusion reactions can deposit their energy in and heat the surrounding fuel. The surrounding fuel will then also undergo fusion, even though it was not initially at a high enough temperature. This sustained burn of the fuel is known as ignition.

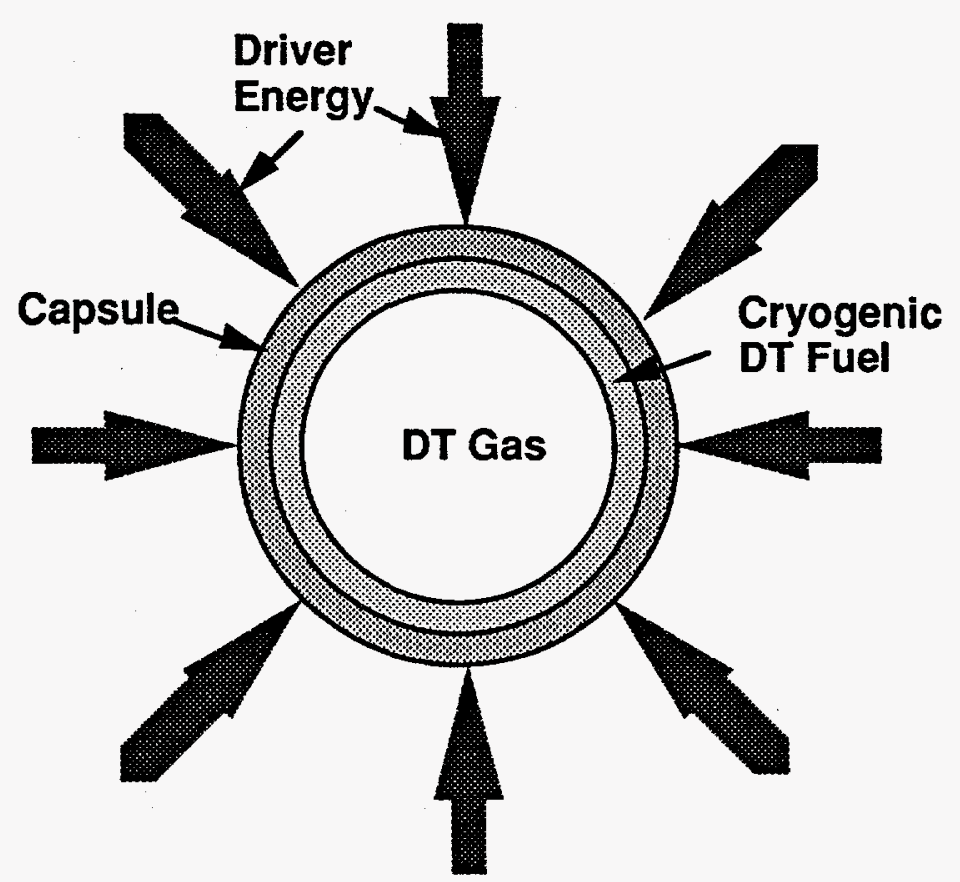

Figure 1-1. Driver energy causes the implosion of an inertial confinement fusion capsule. 
For a direct-drive target, driver energy proceeds directly from the source to the capsule surface. For an indirect-drive target, driver energy indirectly illuminates the capsule surface with soft (about $300 \mathrm{eV}$ ) $\times$ rays. An example heavy ion beam indirect-drive target designed by Darwin Ho is shown in Fig. 1-2. ${ }^{3}$ The ion beam energy heats the absorber. The absorber then radiates black body radiation which cannot penetrate the lead wall. The entire case fills with soft $x$ rays which cause ablation of the capsule surface. The indirect-drive method can provide better implosion symmetry than the direct-drive method with fewer and less uniformly placed driver beams.

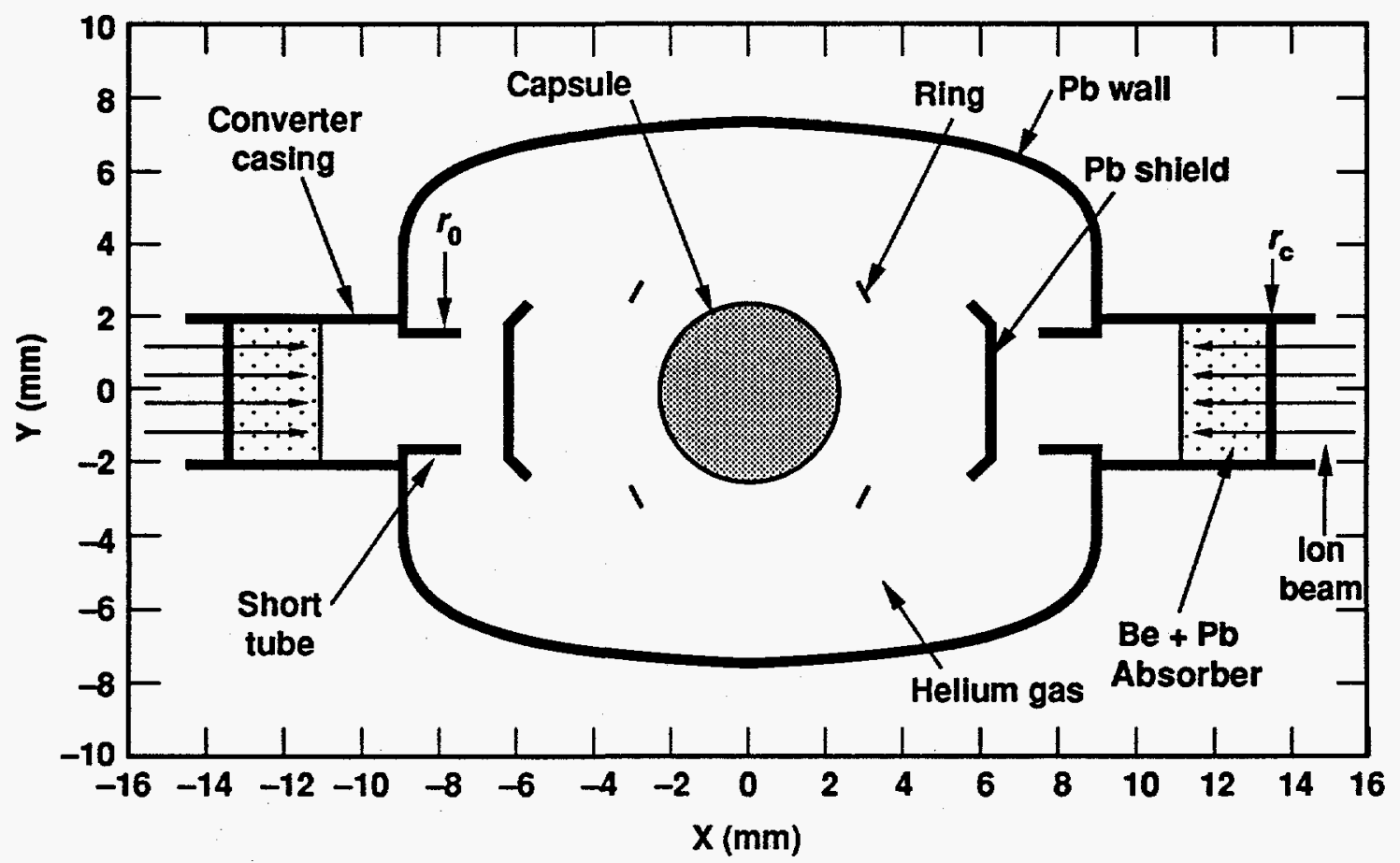

Figure 1-2. Heavy ion indirect-drive target designed by Darwin Ho. ${ }^{3}$ 


\subsection{An IFE Power Plant}

Inertial fusion energy (IFE) normally refers to the use of inertial confinement fusion to produce electrical power. An IFE power plant absorbs energy from fusion micro-explosions in a moving blanket material. The blanket must be thick enough (about $0.5 \mathrm{~m}$ ) to absorb $14 \mathrm{MeV}$ neutrons. The blanket contains lithium, which absorbs neutrons and releases tritium to replace tritium used in fusion reactions. If the blanket flows inside the first structural wall, as in Fig. 1-3, it provides the added benefit of protecting the structural wall from neutron damage. This liquid or granular blanket material is transported to heat exchangers which boil water, or heat some other fluid to drive turbine generators and ultimately produce electricity. A three-dimensional rendering of such a power plant with a recirculating heavy-ion induction accelerator is shown in Fig. 1-4.

(a)

(b)

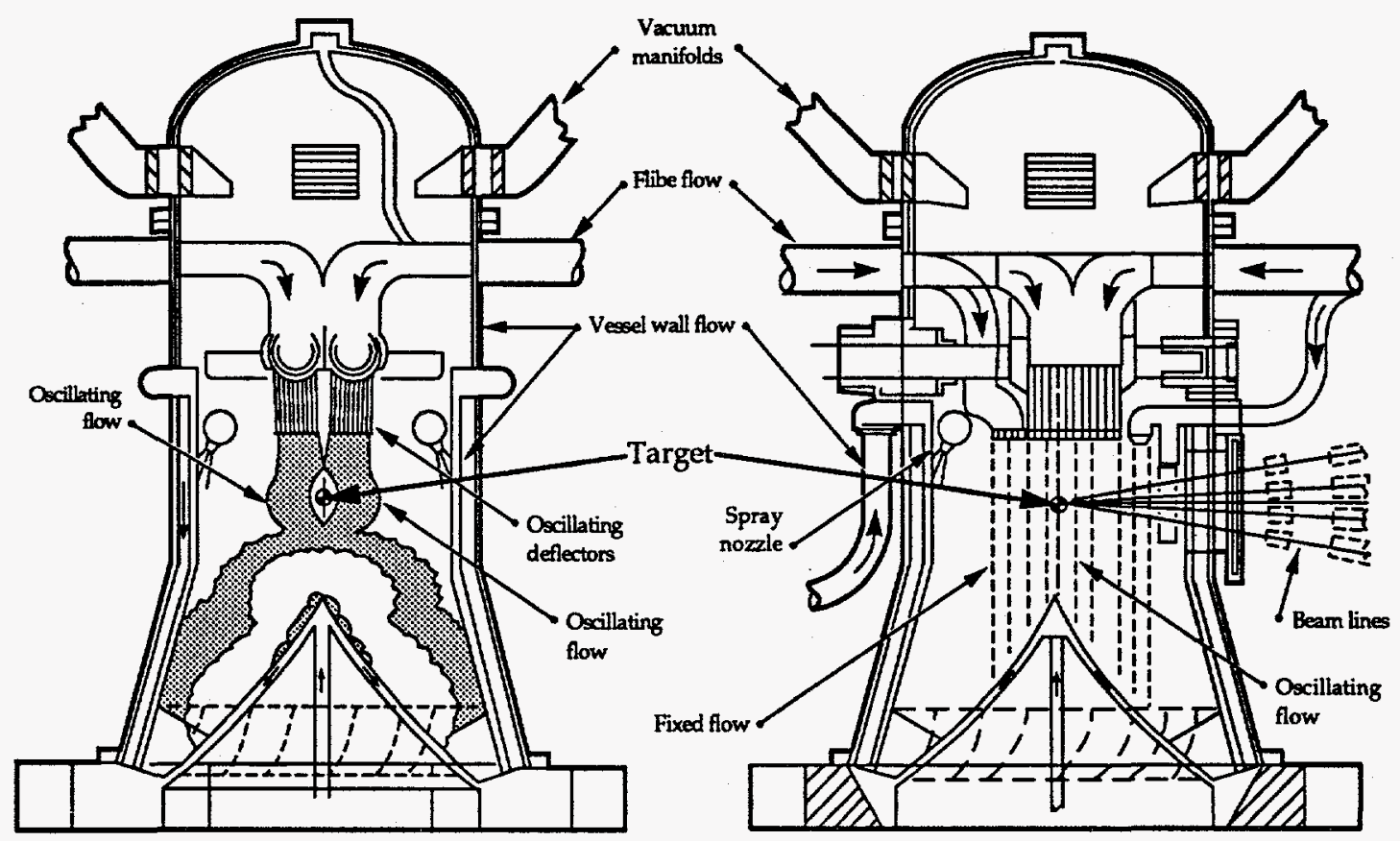


(c)

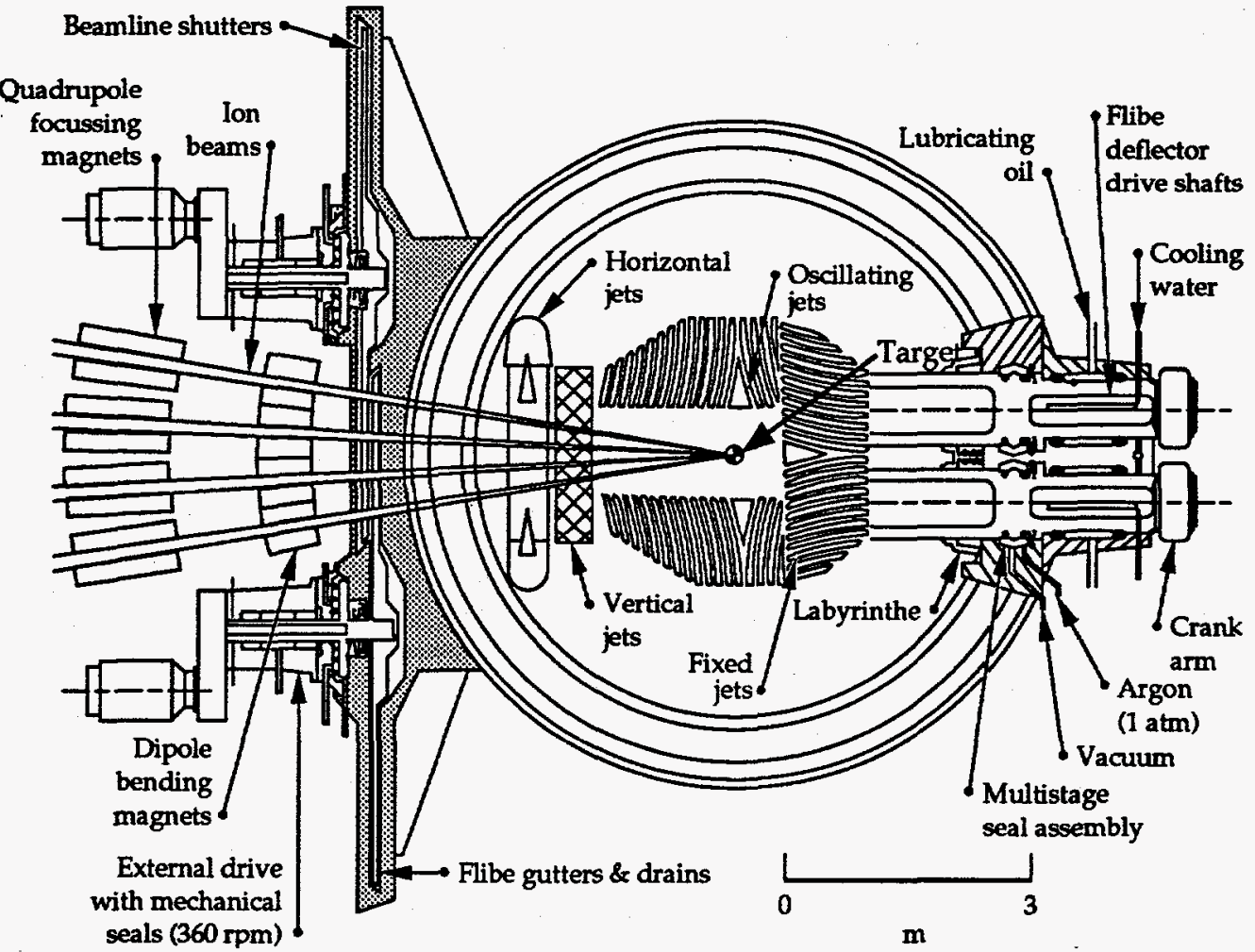

Figure 1-3. (a) Front, (b) side, and (c) top views of the HYLIFE-II reaction chamber. ${ }^{4}$ Ion beam energy is focused on the targets to drive the target implosions. Targets are injected into a "pocket" within a thick oscillating layer of molten salt (called Flibe). The Flibe protects the walls of the chamber from the neutrons, $x$ rays, and debris from the fusion micro-explosions.

A typical gas gun target injection arrangement is shown in Fig. 1-5. Several targets must be injected into the center of an IFE reaction chamber each second. Acceleration forces will mechanically stress the target. To allow driver beams to accurately hit the targets, the targets must be tracked to determine when and where they will pass through the center plane of the chamber. If sufficient injection accuracy is not achieved, it will be necessary to steer the target to the beams, or actively point driver beams at the target. This dissertation examines target injection, tracking, and beam pointing and provides solutions to many of the technically challenging problems. 


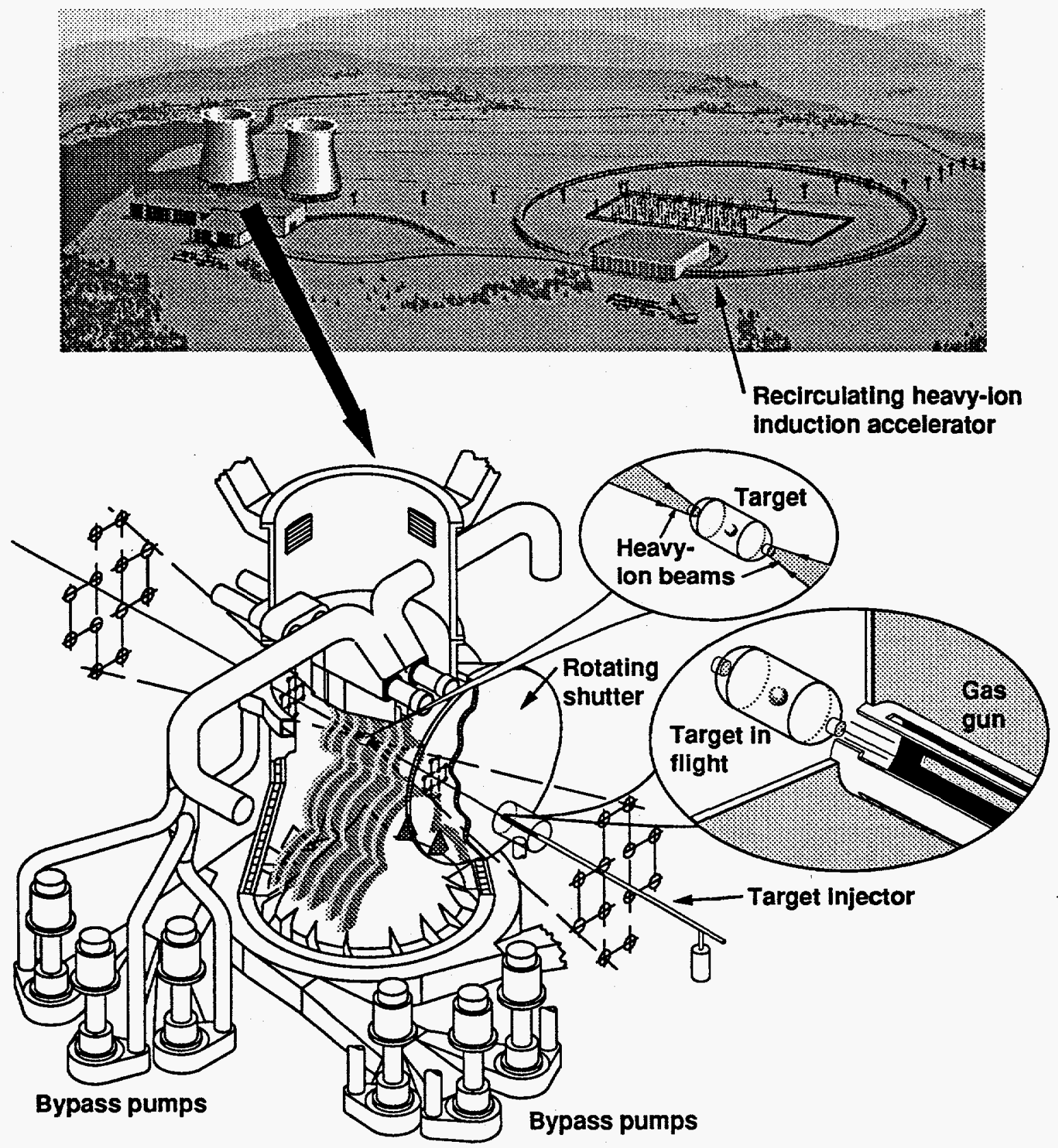

05-00-1194-3846B

16RWiddom

Figure 1-4. Artist's rendering of HYLIFE-II power plant. 


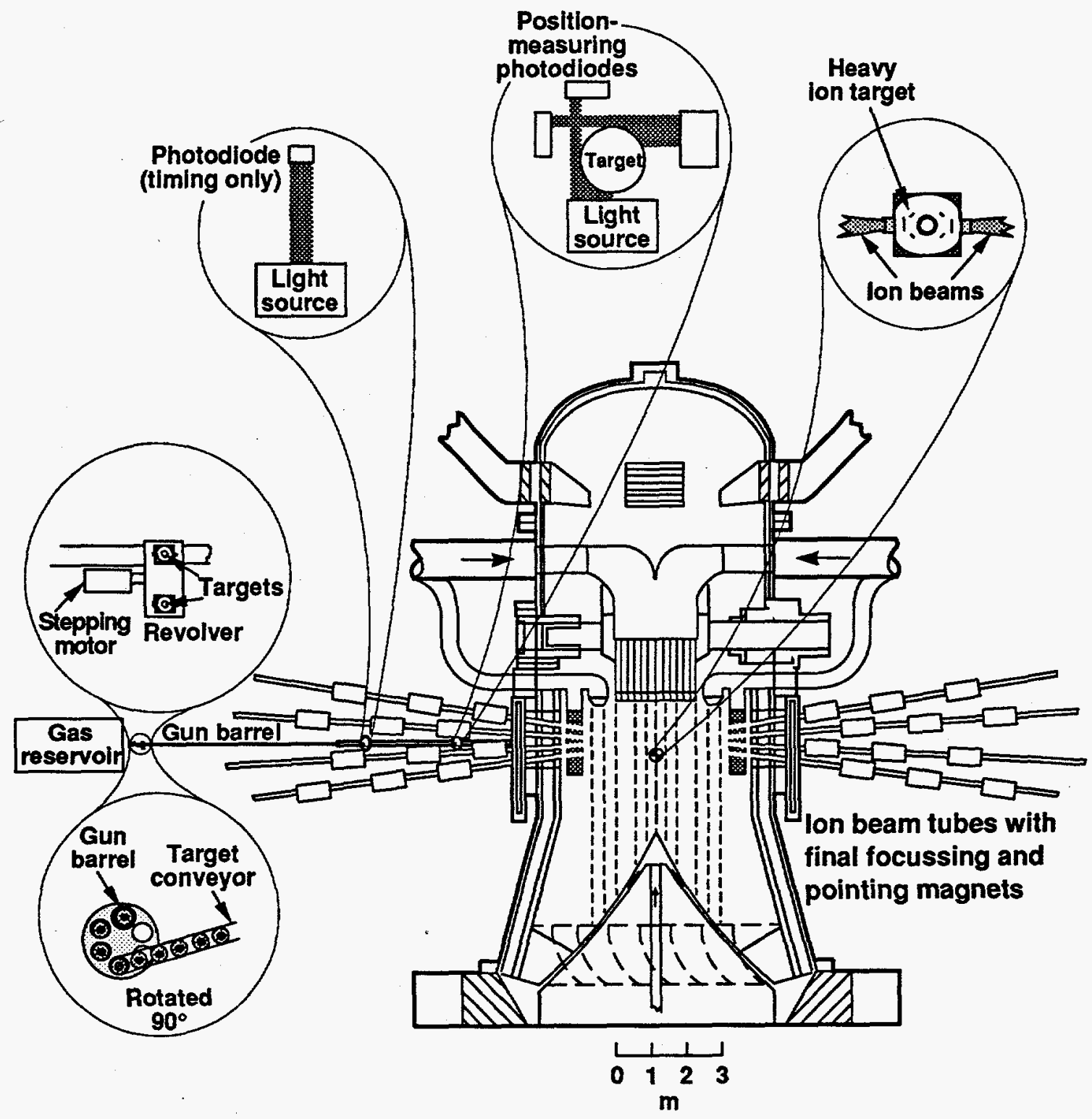

05-00-1194-3690pb01 01GBLinh

Figure 1-5. IFE reaction chamber with target injector and final part of driver beams. 


\subsection{References}

1. R. A. Haas, General Principals of Inertial Confinement Fusion, Lawrence Livermore National Laboratory, Livermore, CA, UCRL-88709 (1983).

2. M. Tabak, Lawrence Livermore National Laboratory, Livermore, CA, private communication (1994).

3. D. D. Ho, J. A. Harte, and M. Tabak, "Radiation-Driven Targets for Heavy Ion Fusion," Presented at the Fifteenth International Conference on Plasma Physics and Controlled Nuclear Fusion Research, Seville, Spain, Sep. 26 - Oct. 2, 1994, IAEA-CN-60/B-P-13.

4. R. W. Moir et al., "HYLIFE-II: A Molten-Salt Inertial Fusion Energy Power Plant Design - Final Report," Fusion Technology, 25(1), 5-25 (1994). 


\section{Chapter 2 - Target Injection. Tracking, and Beam Pointing}

\section{Requirements and Feasibility Considerations}

\subsection{Introduction}

IFE targets are injected into the center of a hot reaction chamber with a pulse rate of about 5 to 10 per second. Target injectors must avoid heating the frozen DT fuel to the triple point (19.7 K) so that the DT remains uniformly frozen within the capsule surface. Additionally the modulus of elasticity and yield stress of DT drop off quickly and the material expands a few percent as temperature rises to the triple point ${ }^{1}$. Since the DT expands at a different rate than the capsule material, stress is caused by this expansion. Therefore, to avoid over-stressing the DT, we may need to limit target heating to a few tenths of a degree. As described in section 2.6, a final fuel temperature of about $18 \mathrm{~K}$ is desirable to provide proper DT gas pressure in the capsule. However, as will be shown in chapter 4 , adequate fuel strength during target acceleration is maintained at temperatures below about $17.4 \mathrm{~K}$. Therefore, it is desirable to operate with as warm a temperature possible while maintaining adequate structural strength of the DT. With indirect drive targets, the hohlraum (radiation container) can protect the capsule from heating and damage in the injector. In the reaction chamber, a few MJ of driver energy (ion beams or laser beams) is focused on the target (see Fig. 1-2). This causes fusion of the fuel in the capsule, releasing a large amount of energy (typically several hundred MJ). To maximize the fusion energy released for a given driver energy, the driver energy must be delivered accurately (to within a few tenths of a millimeter) on the target's surface. This requires accurate knowledge of the target's position and orientation as well as the driver beam positions. 
Targets must be injected many times each second over a distance of about 10 meters. This roughly $10 \mathrm{~m}$ injection distance includes a few meters for target acceleration, a few meters for target tracking and steering and a few meters for passage through the reaction chamber and varies considerably with different power plant designs. The maximum target acceleration is limited by the strength of the targets and the capabilities of the injection system, but is dictated by the pulse rate as well as the reaction chamber size and injector lengths. The interdependent relationships between target acceleration, target speed, reaction chamber size, target injector length, and target coasting distance are investigated in section 2.2.

The accuracy requirements for target injection, tracking, and beam pointing are presented in section 2.3. The methods of tracking and beam pointing are covered in chapter 7. Reliability and cost considerations are briefly covered in section 2.4. A new concept of providing movable or fixed neutron shielding for targets and target injectors is introduced in section 2.5. This allows multiple targets to be in flight at once from a single injector. The use of multiple and therefore redundant target injectors is also presented. The problem of target heating is investigated in section 2.6. Table 2-1 summarizes many of the requirements for target injection, tracking, and beam pointing that are discussed in more detail and justified throughout this chapter. 
Table 2-1. Target injection reference case nominal goals and prognosis for achieving the goals.

\section{Parameter}

-Injection speed

-Target acceleration

-Pulse rate

-Target heating

-Injector shielding

-Total accuracy of beams on target

-Target placement

-Target tracking

-Tumble

-Active beam pointing ${ }^{* 3}$

-Availability

\section{Requirement}

$\sim 100 \mathrm{~m} / \mathrm{s}$

$\sim 2,000 \mathrm{~m} / \mathrm{s}^{2}$

$-6 / 5$

Fuel temp $\sim 17-18 \mathrm{~K}$

$\sim 230 \mathrm{~mm}$ long rod, $\sim 20 \mathrm{~mm}$ diameter

$\pm 0.4 \mathrm{~mm}^{* 1}$ (20\% of beam spot size)

$\pm 0.23 \mathrm{~mm}$ (or $\pm 10 \mathrm{~mm}$ with active beam pointing)

$\pm 0.23 \mathrm{~mm}(= \pm 0.4 \mathrm{~mm} / \sqrt{3})$

$\pm 0.23 \mathrm{~mm}$ ( 1 degree $)$

$\pm 10 \mathrm{~mm}$ with $\pm 0.23 \mathrm{~mm}$ accuracy

$90 \%$ for each, $99 \%$ for two

\section{Achievability}

Exceeded by air guns

Exceeded by air guns

Exceeded by many automatic processes

Achievable

Achievable with movable shielding

Not yet demonstrated ${ }^{* 2}$

$< \pm 1 \mathrm{~mm}$ achieved by air guns at $10 \mathrm{~m}$ range

Optical detection and real time trajectory calculation need demonstration

Spin stabilize if necessary

Achievable but not yet demonstrated

Unknown, needs demonstration

*1 Required accuracy of direct drive beams is about $\pm 0.3 \mathrm{~mm}$ ( $10 \%$ of capsule radius).

*2 A feedback mechanism needs to be developed so that gradual drifts between beam and target positions are measurable and can be corrected.

*3 Active beam pointing may be avoided by in-flight target steering. 


\subsection{Acceleration, Speed, Injection Distance, and Rate}

\section{Relationships}

\subsubsection{Basic Parameters}

This description of target acceleration and speed considerations builds on previous work. 2,3

The target injector (a gas gun for example) accelerates the target for a distance $L_{a}$ after which the target coasts for a distance $L_{c}$ including chamber radius $r$ (Fig. 2-1). A shield/shutter system opens to allow target entry into the reaction chamber but is shut as soon as the target passes by the shutter. The shutter (shown in detail in Fig. 1-3 c) keeps target debris and ablated material away from the target injector and shields the injector and subsequent targets from $x$ rays. The shutter may also be built with sufficient thickness (about $0.23 \mathrm{~m}$ ) to shield the target injector and targets from fusion neutrons.

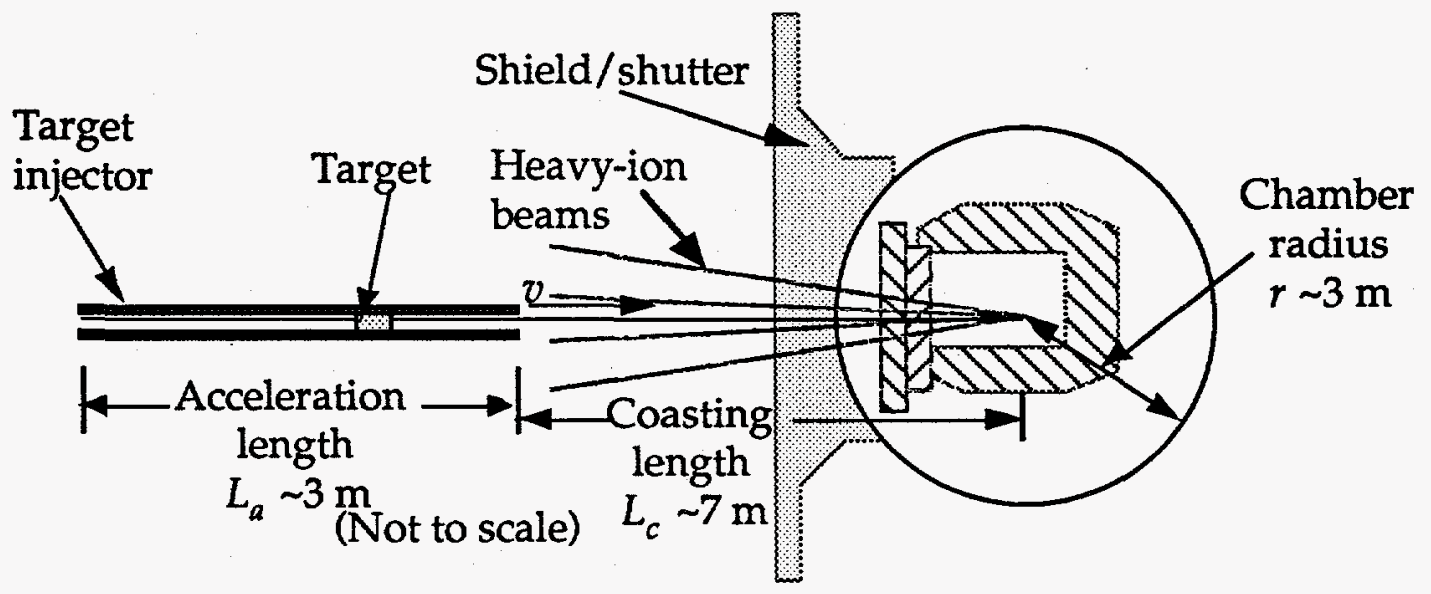

Figure 2-1. The target is accelerated a distance $L_{a}$ through the target injector then coasts a distance $L_{c}$ to the center of the reaction chamber.

The target speed $v$ achieved by accelerating the target a distance $L_{a}$ is given by 


$$
v=\sqrt{2 a L_{a}}
$$

where $a$ is the assumed constant acceleration delivered to the target.

The time $t_{a}$ required for acceleration is given by

$$
t_{a}=\frac{v}{a}=\sqrt{\frac{2 L_{a}}{a}}
$$

The coasting time $t_{c}$ is given by

$$
t_{c}=\frac{L_{C}}{v}
$$

Total target flight time $t$ is given by

$$
t=t_{a}+t_{c}=\frac{v}{a}+\frac{L_{C}}{v}
$$

After the previous micro-explosion, some fraction of the interpulse time $t_{i}$ is required for the reaction chamber to clear sufficiently for target entry. In the absence of more specific information, we often arbitrarily assume that one half of the interpulse time is required for chamber clearing. The remaining time $t_{r}$ is available for the target to travel the radius of the reaction chamber $r$. $t_{r}$ may be further limited by factors such as capsule heating. Excessive capsule heating is undesirable because it would cause premature melting or ablation of the cryogenic fuel or separation of the fuel from the capsule wall. This sets a lower limit on $v$,

$$
v \geq \frac{r}{t_{r}}
$$


Acceleration length is inversely proportional to acceleration,

$$
L_{a}=\frac{v^{2}}{2 a} .
$$

The maximum acceleration that a target can undergo without damage is shown in chapter 3 to be about $1,800 \mathrm{~m} / \mathrm{s}^{2}$ for a $0.1 \mu \mathrm{m}$ thick dual membrane example. Greater acceleration would be possible with thicker membranes, but this could lead to unacceptable implosion symmetry.

As indicated in Fig. 2-2, both the maximum acceleration that a target can undergo without damage and the minimum target speed (from equation 2-5) limit the parameter range that may be used for target injection. The available operating region includes those combinations of target speed and acceleration for which it is possible to inject targets while meeting imposed requirements.

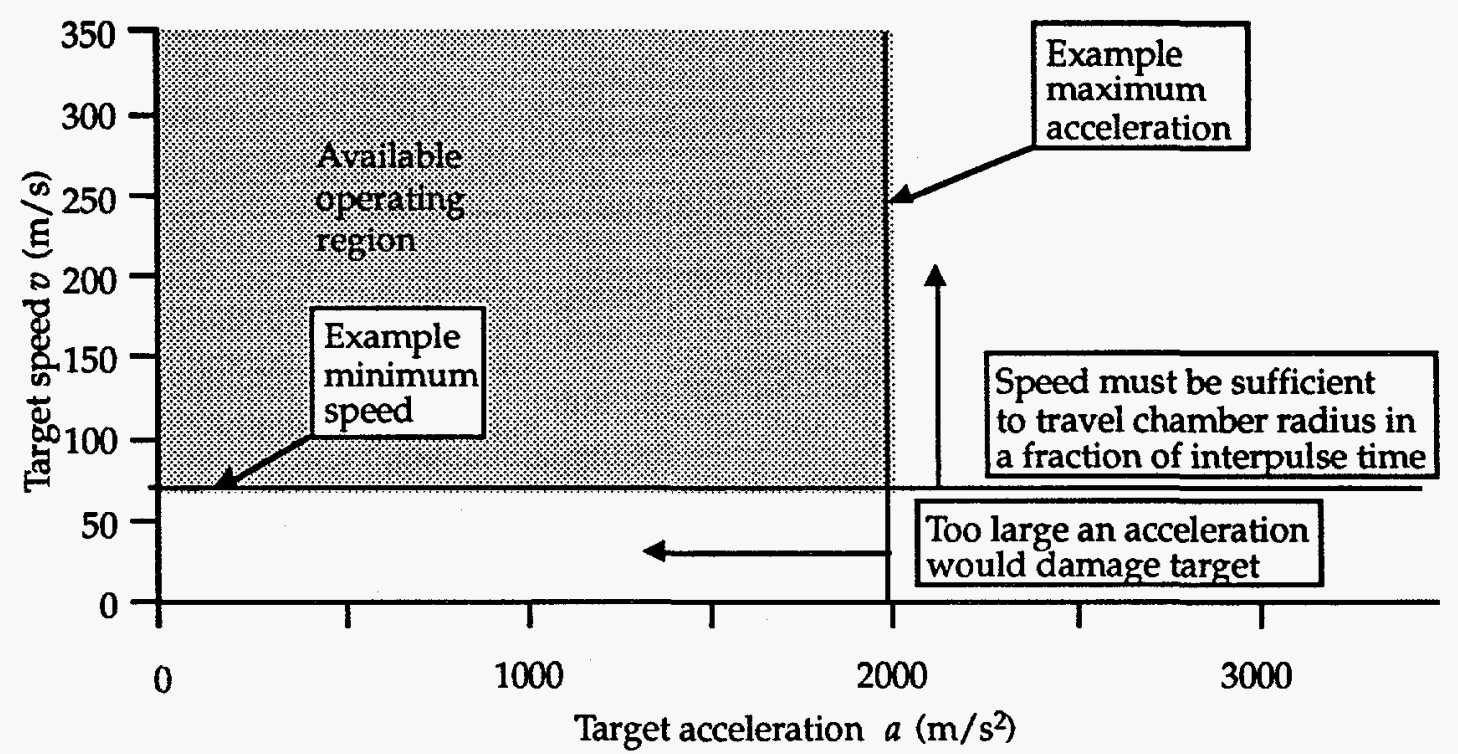

Figure 2-2. Speed and acceleration limits for target injection. 
Figure 2-3 shows the quantitative relationship between target speed and acceleration for various acceleration lengths. Recent power plant design points are included as examples 2,4,5,6. HYLIFE-II has a $3 \mathrm{~m}$ chamber radius and a $6 \mathrm{~Hz}$ pulse rate. HYLIFE-II uses a falling molten salt first wall to absorb the fusion energy and reduce activation of reaction chamber structural material. For HYLIFE-II the assumed acceleration is $1,000 \mathrm{~m} / \mathrm{s}^{2}$ with desired target speed of $100 \mathrm{~m} / \mathrm{s}$. With the $100 \mathrm{~m} / \mathrm{s}$ injection speed, target time in the chamber is limited to $30 \mathrm{~ms}$. This allows $137 \mathrm{~ms}$ for the splashing molten salt to be cleared from the target path. From equation (2-6), the required acceleration length is calculated to be $5 \mathrm{~m}$. SOMBRERO has a $7.5 \mathrm{~m}$ radius chamber and assumes only $1 / 3$ of the $150 \mathrm{~ms}$ interpulse time is available for the target be in the reaction chamber. SOMBRERO assumes a maximum target acceleration of $1300 \mathrm{~m} / \mathrm{s}^{2}$. OSIRIS is a $4.6 \mathrm{~Hz}$ indirect drive power plant with the same target speed and acceleration design point as SOMBRERO. 3 Prometheus- $\mathrm{H}$ is a $4.5 \mathrm{~m}$ radius chamber indirect drive power plant with 100 $\mathrm{g}$ target acceleration and $200 \mathrm{~m} / \mathrm{s}$ injection speed requiring a $20 \mathrm{~m}$ acceleration length. 5 Prometheus- $\mathrm{L}$ is a $5 \mathrm{~m}$ radius chamber direct drive power plant with a much larger acceleration of $10,000 \mathrm{~m} / \mathrm{s}^{2}$ and injection speed of $200 \mathrm{~m} / \mathrm{s}$ (not plotted on Fig. 2-3). 5 An estimated design point for Cascade is included for comparison. The Cascade ICF reaction ${ }^{6}$ chamber is $8.6 \mathrm{~m}$ long and has an interpulse time of 0.2 seconds. Assuming $0.1 \mathrm{~s}$ (half of the interpulse time) is available for the target to traverse $4.3 \mathrm{~m}$ through the reaction chamber, the minimum target speed from equation (2-5) would be $43 \mathrm{~m} / \mathrm{s}$. Assuming 1,000 $\mathrm{m} / \mathrm{s}^{2}$ acceleration is possible for cascade targets, the acceleration length from equation (2-6) would be $0.92 \mathrm{~m}$. As a result of the above survey, we have chosen $v=100 \mathrm{~m} / \mathrm{s}$ and $a=2,000 \mathrm{~m} / \mathrm{s}^{2}$ as nominal reference values for this dissertation. 


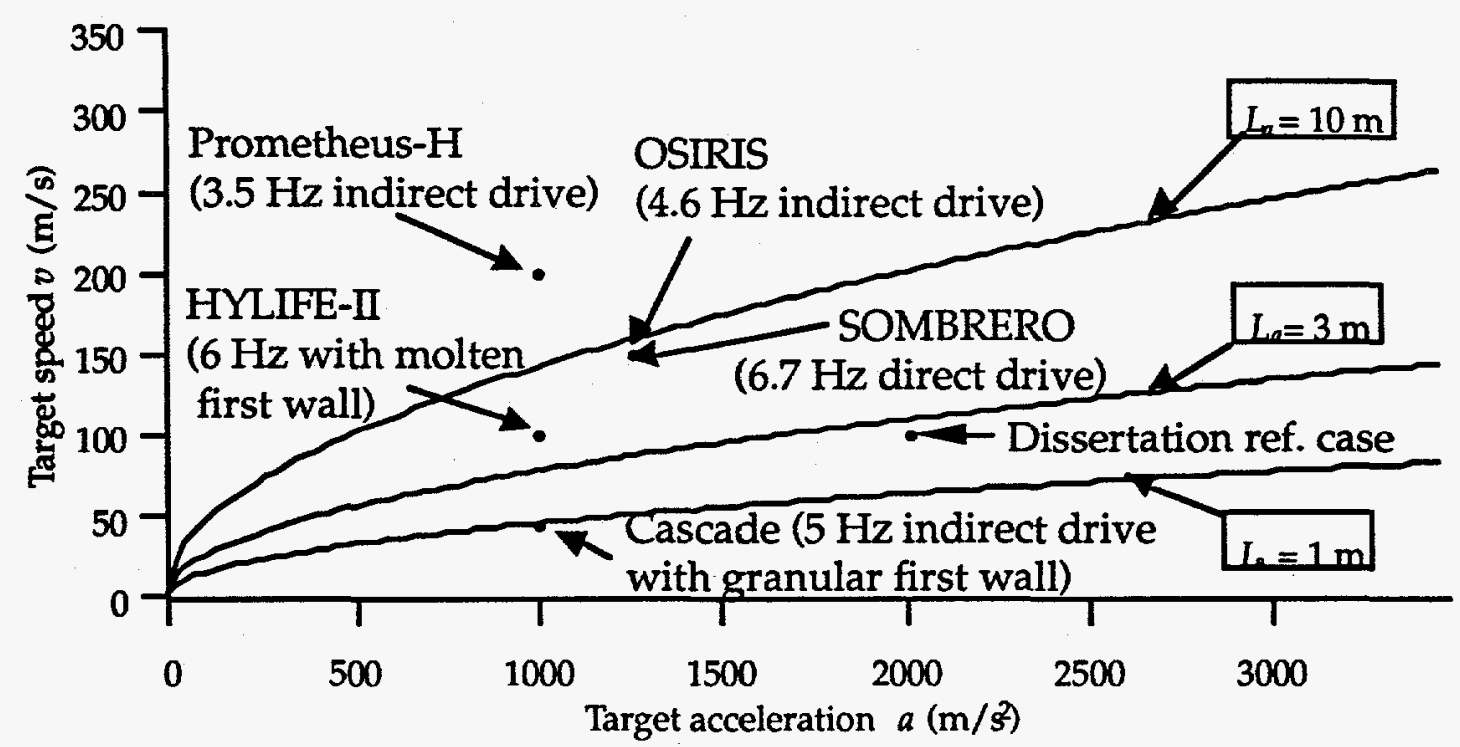

Figure 2-3. Target speed vs. acceleration for acceleration lengths $L_{a}=1 \mathrm{~m}, 3 \mathrm{~m}$, and $10 \mathrm{~m}$. The HYLIFE-II, SOMBRERO, OSIRIS, Prometheus-H, estimated Cascade injector, and our reference design points are included.

\subsubsection{Implications of Assuming Target Acceleration Time is Less than}

\section{Interpulse Time}

If more than one target is to be in motion toward the chamber center at one time, shielding must be provided to protect targets from the neutrons, $x$ rays, and debris from the previous targets micro-explosion. Further study is required to determine how much of what kind of shielding is required. However, section 2.5 indicates that about $1 / 4 \mathrm{~m}$ of neutron shielding is probably required. There are several methods of providing neutron shielding for targets and target injectors. This neutron shield may be a thin cylindrical bar (i.e. a few $\mathrm{cm}$ diameter as indicated in Fig. 2-9) which is moved into place after the target enters the reaction chamber. It could be a liquid shield injected in the neutron line of sight. Or, for slower moving targets with relatively long coasting distance the parabolic path of the target may allow fixed shielding to do the job. With neutron shielding, a reasonable assumption 
might be that we have only one target injector and that only one target may be accelerated at a time (one target in gun at a time, i.e. target acceleration time must be less than the interpulse time). The target speed $v$ is simply given by

$$
v=a t_{a}
$$

Since acceleration time $t_{a}$ is less than the interpulse time, target speed can never be greater than acceleration times the interpulse time $t_{i}$.

$$
v \leq a t_{i}
$$

To calculate a target injector design point, some operating parameters need to be known. For simplicity, assume the maximum acceleration the target can undergo without damage and the maximum acceleration the injector can provide are known. To minimize injector length, the lesser of these two limiting accelerations will normally be used (This is an oversimplification. The maximum target acceleration and maximum acceleration a target injector can provide are design variables that must be optimized in a system cost-benefit study). The distance $r$ that the target must travel through the reaction chamber and the allowed time $t_{r}$ in the chamber must be known. $t_{r}$ is usually limited by target heating or by the interpulse time less the time required for chamber conditions to be suitable for target entry.

With the above information (which will be different for each power plant and target design), calculating the design point target speed, acceleration, and injector length is straightforward. Simply use inequality (2-5) to calculate the minimum target speed and equation (2-6) to calculate the injector length. 
Then use inequality (2-8) to verify the acceleration is adequate to ensure only one target is accelerated at a time.

Maximum speed vs. acceleration is plotted in Fig. $2-4$ for a 4, 6, and $8 \mathrm{~Hz}$ pulse rate. Also plotted on Fig. 2-4 is speed vs. acceleration for various acceleration lengths (from equation 2-1). The maximum acceleration and minimum speeds are taken from the following example.

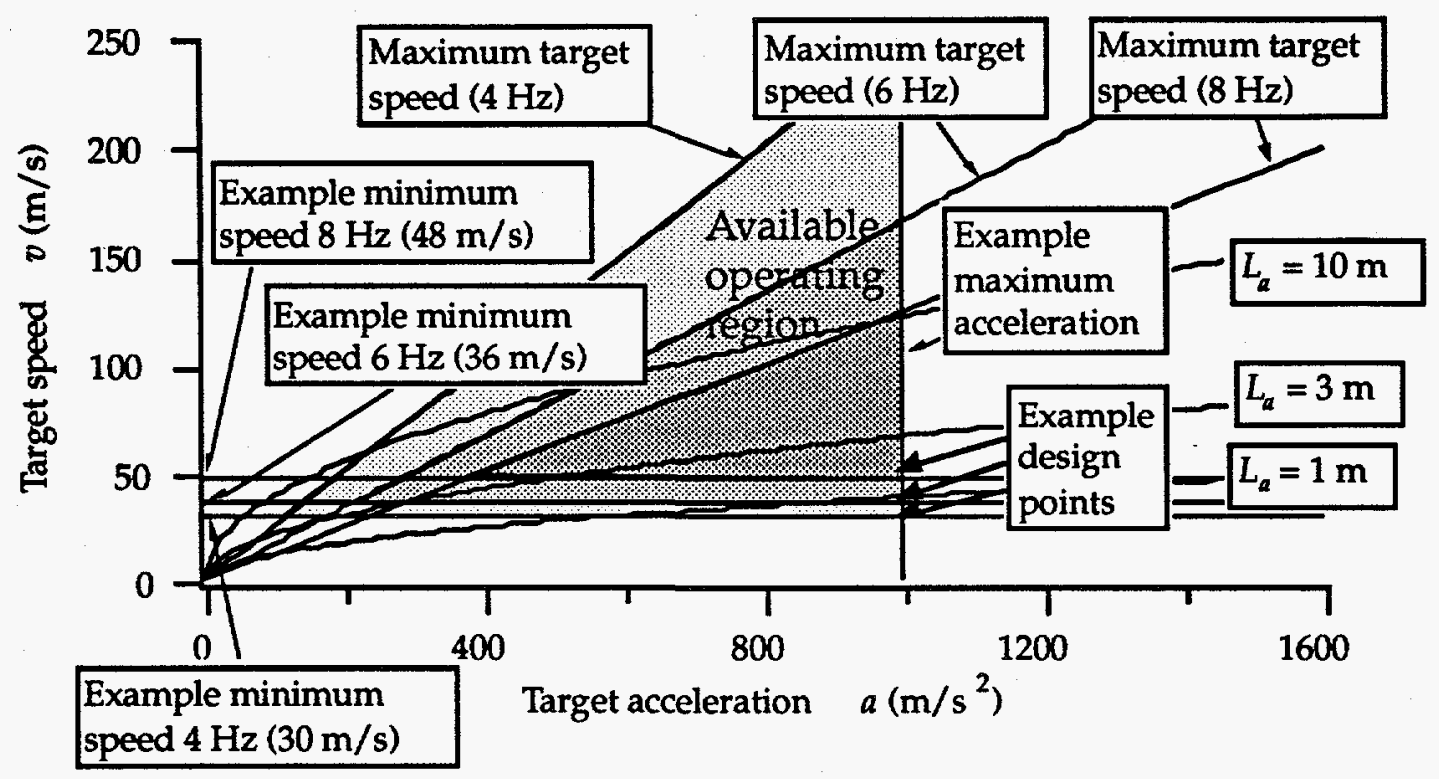

Figure 2-4. Target speed vs. acceleration is plotted. Available operating ranges are shaded for $4 \mathrm{~Hz}, 6 \mathrm{~Hz}$, and $8 \mathrm{~Hz}$ pulse rates. Minimum speeds, maximum acceleration, and design points apply to the following example. Target speed vs. acceleration is also indicated for acceleration lengths of $1 \mathrm{~m}, 3 \mathrm{~m}$, and 10 $\mathrm{m}$. This figure assumes only one target is accelerated at a time. 
Example problem for target injector design point (one target accelerated at a time)

For the purpose of providing a specific example, suppose a power plant design has the following arbitrary parameters. The chamber radius is $3 \mathrm{~m}$, with a coasting distance $L_{c}$ of $5 \mathrm{~m}$, and the targets would be damaged by accelerations greater than $1,000 \mathrm{~m} / \mathrm{s}^{2}$. This example acceleration is less than our reference acceleration of $2000 \mathrm{~m} / \mathrm{s}^{2}$ but the resulting calculations are completely analogous. Further suppose that the target minimum speed is limited by two factors:

1. Due to reaction chamber conditions, the target is assumed to not enter the reaction chamber for the first half of the interpulse time. An $8 \mathrm{~Hz}$ pulse rate ( $0.125 \mathrm{~s}$ interpulse time) would give $0.062 \mathrm{~s}$ chamber transit time with

$$
v \geq \frac{r}{t_{r}}=\frac{3 \mathrm{~m}}{0.062 \mathrm{~s}}=48 \mathrm{~m} / \mathrm{s} .
$$

Similarly, $4 \mathrm{~Hz}$ would require a minimum speed of $24 \mathrm{~m} / \mathrm{s}$ and $6 \mathrm{~Hz}$ would require a minimum speed of $36 \mathrm{~m} / \mathrm{s}$.

2. Also assume that due to capsule heating, the capsule must be in the reaction chamber less than $0.1 \mathrm{~s}$ (target heating rates vary with target and chamber design as discussed in section 2.6). This gives

$$
v \geq \frac{r}{t_{r}}=\frac{3 \mathrm{~m}}{0.1 \mathrm{~s}}=30 \mathrm{~m} / \mathrm{s} .
$$

Making the above listed assumptions, suppose the proposed design incorporated a $4 \mathrm{~Hz}$ pulse rate. We now follow the procedure described above 
to calculate and verify adequacy of the design parameters. The design (minimum) target speed is $30 \mathrm{~m} / \mathrm{s}$ (the greater of $24 \mathrm{~m} / \mathrm{s}$ and $30 \mathrm{~m} / \mathrm{s}$ ). The minimum target speed and maximum target acceleration are used to minimize target injector length and improve ease of tracking the targets. The example acceleration is $1,000 \mathrm{~m} / \mathrm{s}^{2}$ which is greater than the minimum acceleration given by equation (2-8).

$$
a \geq \frac{30 \mathrm{~m} / \mathrm{s}}{0.25 \mathrm{~s}}=120 \mathrm{~m} / \mathrm{s}^{2}
$$

The injector length is calculated with equation (2-6).

$$
L=\frac{v^{2}}{2 a}=\frac{(30 \mathrm{~m} / \mathrm{s})^{2}}{2\left(1000 \mathrm{~m} / \mathrm{s}^{2}\right)}=0.45 \mathrm{~m}
$$

The design point and the entire available operating region are shown on Fig. 2-4.

Similar calculations were accomplished for a $6 \mathrm{~Hz}$ and a $8 \mathrm{~Hz}$ pulse rate. The results of these calculations are also indicated on Fig. 2-4. The available operating ranges are smaller with higher pulse rates.

\subsubsection{Implications of Using Multiple Injectors}

Excessive acceleration would structurally damage any target. The capsule support membrane could be broken or the DT could become separated from the inner capsule surface. If target damage thresholds or other factors require acceleration that is below the minimum for a required target speed, there are at least two possible solutions. One is to reduce the pulse rate. A better solution might be to use more than one target injector. For example, with three injectors, one target could be loaded while a second is in an early stage 
of acceleration, a third is in a later stage of acceleration, and others are coasting toward the chamber center (see Fig. 2-5). As discussed in section 2.5, target shielding is used to minimize target heating.

With two injectors, more time is available to accelerate targets, so the maximum target speed attainable, given by equation (2-8), is doubled. Or equivalently, the required minimum acceleration is cut in half. The minimum target speed given by equation (2-5) and the maximum acceleration are unchanged.

Perhaps, the most important reason for having more than one injector is improved system reliability. Even if a single target injector is capable of delivering targets with adequate speed and rate to a reaction chamber, it would be desirable to have more than one for redundancy. Two injectors could each be operated at one half capacity. Then failure of a single injector would not require immediate shutdown of the power plant. The other injector could double its target output. The acceleration and speed of the targets would not change, only the interval between targets in the remaining injector. The obvious disadvantage of having two injectors rather than one is the increased cost associated with two injector systems. 


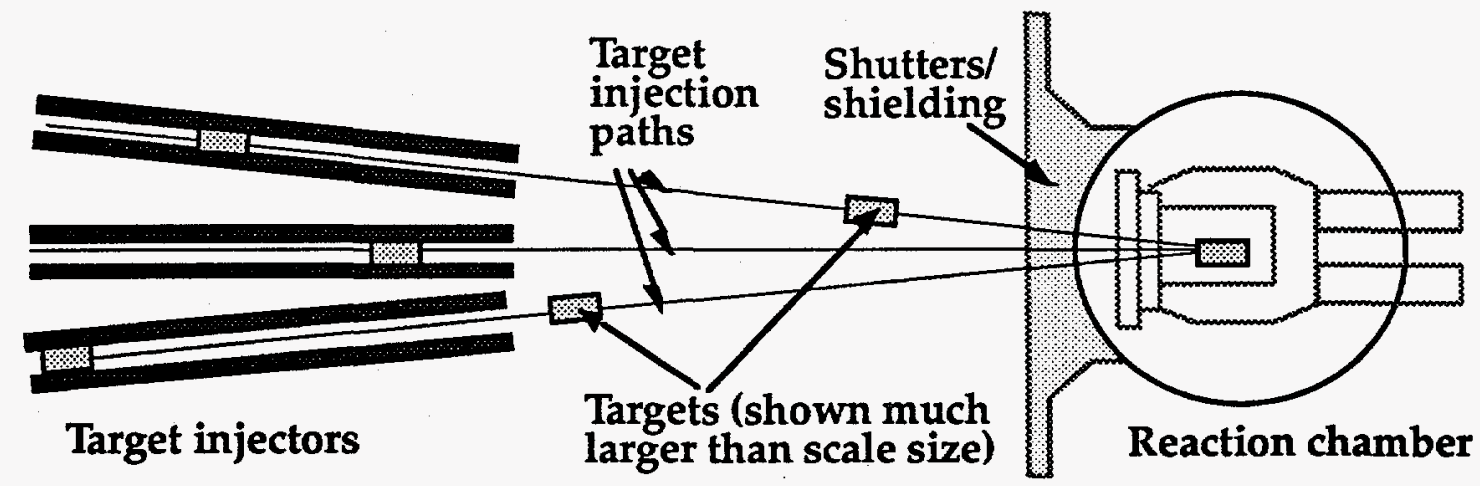

Figure 2-5. Multiple target injectors may be necessary for some power plant designs. Multiple injectors may also be desirable to enhance power plant reliability.

\subsection{Accuracy Requirements}

Target gain (the ratio of fusion energy to driver energy) is dependent on how accurately the driver beams hit the target. Reference 3 (appendix A) gives accuracy requirements for the leading fusion energy candidate (direct laser driven and indirect ion driven) targets.

\subsubsection{Direct Drive}

For direct laser driven targets, the root mean square variation in beam intensity should be less than $1-2 \%$. This leads to an accuracy requirement for beam positioning on the target of one tenth of the capsule radius. For a typical capsule radius of $3 \mathrm{~mm}$, the total error must be less than $\pm 0.3 \mathrm{~mm}$. This error must be shared between the target tracking error and the beam pointing error. These errors add in quadrature and assuming equal errors are allowed for each, the allowed errors would be $\pm 0.3 \mathrm{~mm} / \sqrt{2}= \pm 0.21 \mathrm{~mm}$. 


\subsubsection{Indirect Drive}

As shown in Fig. 2-6a, indirect drive heavy-ion targets have absorber/radiators onto which the ions are focused. The radius of the spot to which the beams are focused is called the spot size. But if the target is mispositioned or rotated relative to the beams, the beams would miss the absorber unless the absorber was made larger to compensate. In either case, target gain decreases. Reference 3 (Appendix A) recommends a maximum inaccuracy of $\pm 20 \%$ of the spot size. For a spot size of $2 \mathrm{~mm}$ (as is used in HYLIFE- $\mathrm{II}^{4}$ ) this gives an allowed error of $\pm 0.4 \mathrm{~mm}$. This error is composed of target tracking error, tumble error, and beam pointing error. These errors add in quadrature and assuming equal errors are allowed for each, the allowed errors would be $\pm 0.4 \mathrm{~mm} / \sqrt{3}= \pm 0.23 \mathrm{~mm}$.

Axial target positioning is achieved by timing the driver beams to meet the target. With a typical heavy-ion driven power plant the multiple driver beams converge from a fairly narrow angle of about $\pm 9^{\circ}$ to the minimum spot size and then start diverging until the target is hit. ${ }^{4}$ If the target is not properly positioned in the axial direction the spot size will grow by roughly one sixth of the axial position error for a $\pm 9^{\circ}$ beam convergence angle. In this case target gain is only about one sixth as sensitive to axial position error as to lateral position error. Allowed axial target position error is then $\pm 0.23 \mathrm{~mm} \times 6 / \sqrt{3}= \pm 0.8 \mathrm{~mm}$. At $100 \mathrm{~m} / \mathrm{s}$ target speed, the allowed timing error would be $\pm 8 \mu$ s. 

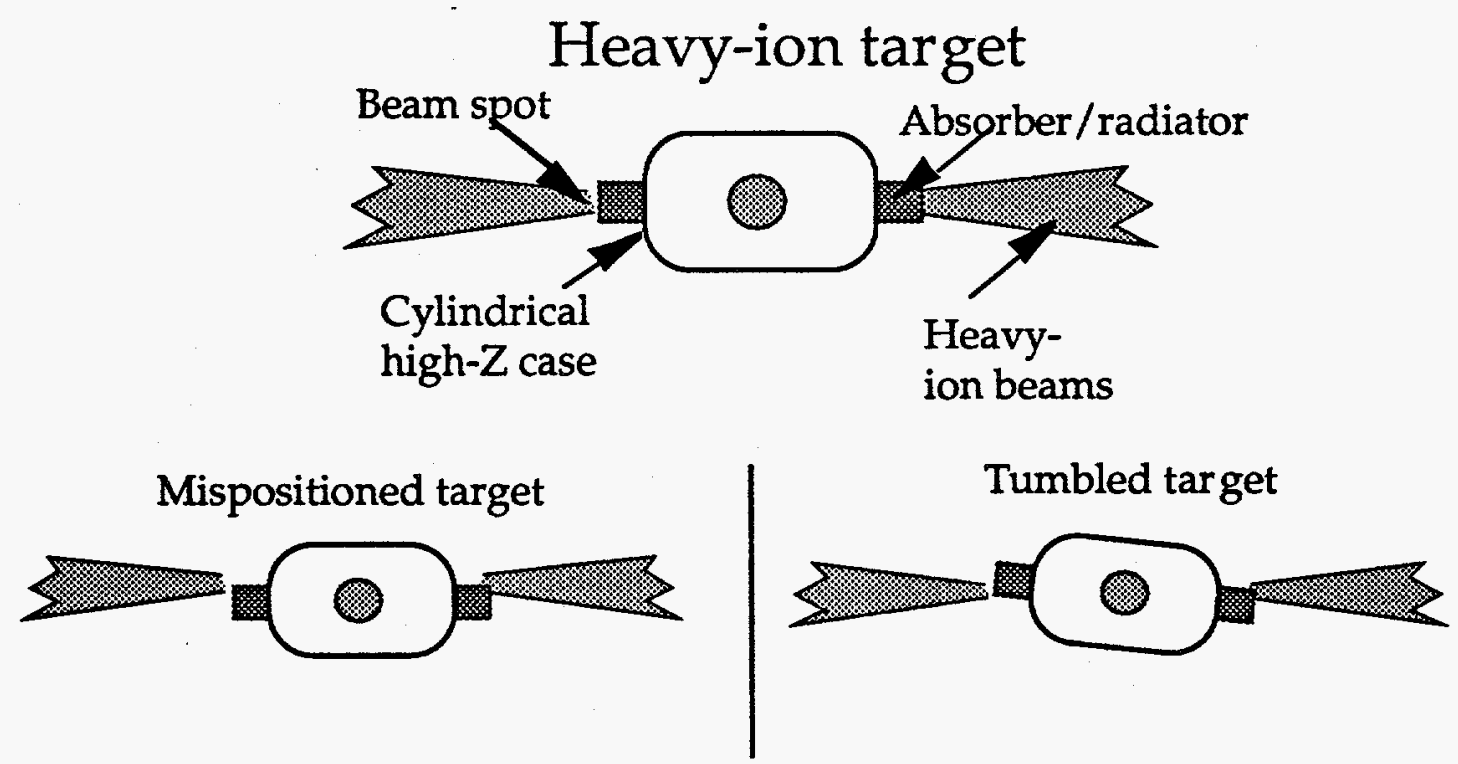

Figure 2-6a. With an indirect ion driven target, ions are focused to a spot to heat absorber/radiator material. If the target is excessively mispositioned or rotated relative to the driver beams, the beams will miss the absorber.

To avoid having to bend driver beam paths $90^{\circ}$ for two-sided illumination, and to simplify power plant reaction chamber design, it may be desirable to use one-sided target illumination as indicated in Fig. 2-6b.4 The target accuracy requirements are unchanged, but the implications of these requirements are different. The target velocity would probably still be along the target axis and would therefore be perpendicular to the driver beam axis. ${ }^{1}$ The factor of six relaxation in allowed target axial position error found in the previous paragraph would no longer be appropriate. The allowed timing error for a $100 \mathrm{~m} / \mathrm{s}$ target velocity would be reduced from $\pm 8 \mu \mathrm{s}$ to $\pm 1.3 \mu \mathrm{s}^{2}$ Of course, greater position and tumble error could be allowed in the transverse direction along the beam paths (Tumble A in Fig. 2-6b). However, allowed

\footnotetext{
${ }^{1}$ For some power plant designs, such as HYLIFE-II, it may be desirable to shoot targets in with velocity perpendicular to the target axis. Targets can be shot through a barrel sideways if the target has edges that conform to the barrel. Material can be added to the target exterior to give the target the desired shape. Alternatively, the target could be placed in a removable sabot. 2 If this $\pm 1.3 \mu$ s timing is not achievable, it may be possible to shoot the targets in with the injection axis perpendicular to the target axis, thereby increasing the allowed timing error.
} 
position and tumble error would still be more limited in the other transverse direction (Tumble B in Fig. 2-6b). If tumble is excessive, spin stabilization could still be used (as described in section 2.3.3). The total number of target rotations that will be required depends on the tumble rate, as described in section 2.3.3. Only a few rotations (if any) are likely to be required in roughly $100 \mathrm{~ms}$ leading to a spin rate of a few tens of revolutions per second. The number of rotations must be controlled so that the beam absorber is properly positioned to face the beam. The allowed error in spin angle $\Delta \psi$ would be given by

$$
\Delta \psi= \pm \sin ^{-1}\left(\frac{0.2}{3} \frac{r_{0}}{r_{2}}\right) \approx \pm \frac{0.2}{3} \frac{r_{0}}{r_{2}}
$$

where $r_{0}$ is the absorber radius, and $r_{2}$ is the distance off axis to the beam side of the absorber. The factor of $0.2 \mathrm{r}_{0}$ comes from the requirement of the beam center hitting the absorber center within $\pm 20 \%$ of the spot size. The divisor 3 comes for statistically dividing the error between 3 axes and three contributions (position, tumble, and beam pointing). For typical values of $r_{0}=$ $2 \mathrm{~mm}$ and $\mathrm{r}_{2}=4 \mathrm{~mm}, \Delta \psi$ is limited to $\pm 1.9^{\circ}$. Allowed tumble angles $\theta$ and $\phi$ around the other two axes can be calculated in a similar manner. The results are

$$
\phi=\frac{0.2 r_{0}}{3 r_{1}} \text { and } \theta=\frac{0.2 r_{0}}{3 r_{1} \sin \gamma}
$$

where $\gamma$ is the effective convergence and divergence angle of the driver beams about the focal spot. 


\section{Single-sided illumination target}
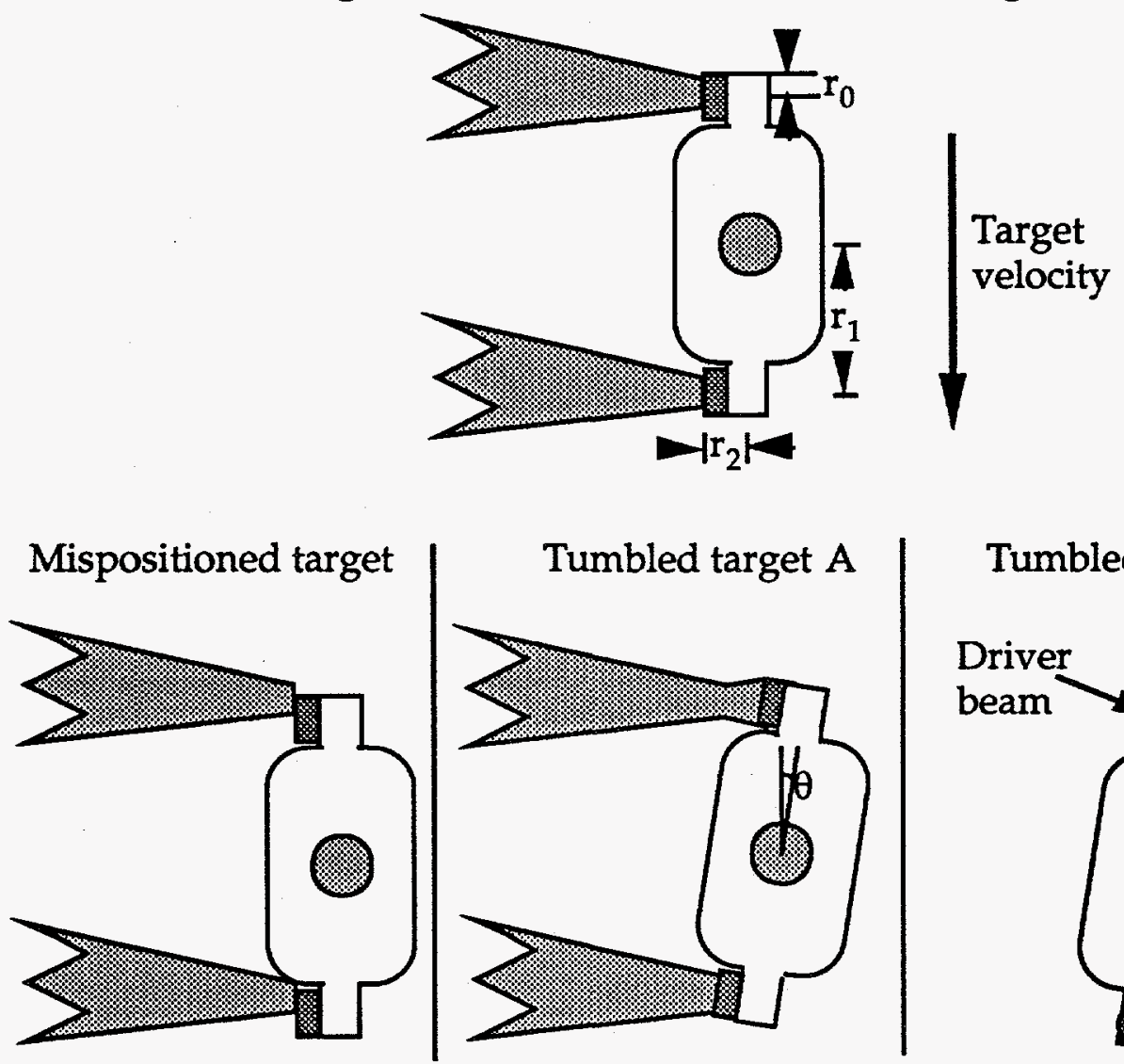

Tumbled target B

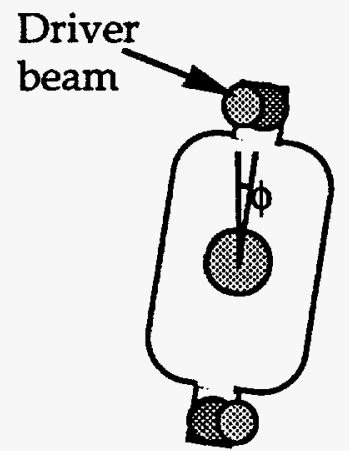

Figure 2-6b. With single-sided illumination and axial target velocity, the axial target position tolerance is reduced, and spin rate must be precisely controlled.

As a benchmark for the accuracy we might expect from a target injector, consider the performance of match grade air guns ${ }^{7}$. At $10 \mathrm{~m}$ (which is a typical target injection distance) "test groups ${ }^{3}$ subtending $1 / 2$ minute of angle are common". $1 / 2$ minute of angle is $145 \mu \mathrm{rad}$ and at $10 \mathrm{~m}$ is $\pm 0.7 \mathrm{~mm}$. Target injectors can probably match or exceed this accuracy since they are shooting into a vacuum and do not rely on how steadily a marksman can hold a gun. If random variation in target position is more than about $\pm 0.2 \mathrm{~mm}$, then the beams will have to be actively pointed toward a different target position on

\footnotetext{
${ }^{3} \mathrm{~A}$ test group is a grouping of holes in a rifle target made while checking the accuracy of the
} rifle. 
each shot, or the target steered toward the beams aim point. One can adjust the pointing of the driver beams to accommodate inconsistent target positioning, however it may be desirable to steer the target (see chapter 5) rather than the beams.

\subsubsection{Gyroscopic Target Stabilization}

As discussed in section 2.6 .3 , spinning the target may require radially placed membranes to keep target gas spinning with the target. The rate of rotation required to maintain axial alignment of the target depends primarily on two factors. First, what is the alignment tolerance? Second, how much tumbling would be expected without rotation stabilization?

The tolerance is based in part on the required beam pointing accuracy. Part of the uncertainty in the location of the part of the target that the driver beams must hit is due to the misalignment of the target. Consider for example an indirect drive heavy-ion target as shown in Fig. 2-7. Let $\theta_{\max }$ be the maximum allowed tumble angle. $\theta_{\max } \approx 2 X / L$ where $X$ is the maximum allowed tumble error and $L$ is the target length. For a typical $18 \mathrm{~mm}$ long target with tumble error $0.23 \mathrm{~mm}$, the maximum tumble angle is $0.026 \mathrm{rad}$ $\left(1.5^{\circ}\right)$. For $100 \mathrm{~ms}$ coasting time, this corresponds to a $0.26 \mathrm{rad} / \mathrm{s}$ tumble rate.

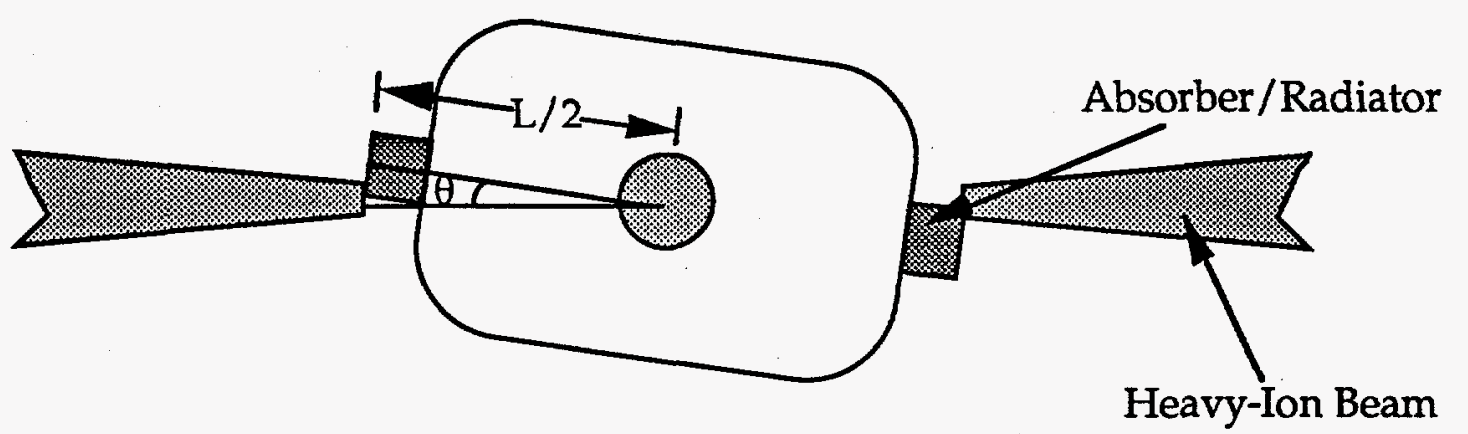

Figure 2-7. Indirect drive heavy-ion beam target rotated an angle $\theta$ from the desired axis. 
If the target tumble rate is such that $\theta$ is less than $\theta_{\max }$ for all targets injected, then there would be no need to spin stabilize the targets. However, if $\theta$ is often greater than $\theta_{\max }$, then spin stabilization could help reduce $\theta$. Figure 2-8 shows the geometry for the precession of a target which is assumed to be a rigid body with an axis of symmetry and no applied forces. The target has angular velocity (spin rate) $\Omega_{3}$ about its axis of symmetry, angular velocity (tumble rate) $\Omega_{1}$ perpendicular to its axis of symmetry, and is precessing with angular velocity $\Omega_{\mathrm{pr}}$ about the direction of its total angular momentum $\mathrm{M}$. The precession angle is $\phi$.

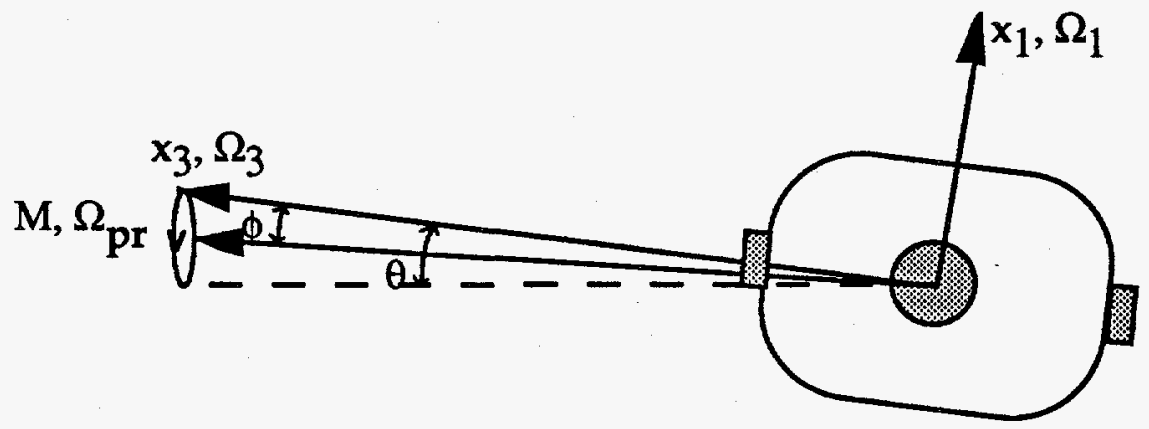

Figure 2-8. A spinning target precesses as it travels through the injection path.

The precession angle $\phi$ is of considerable importance for target injection. As indicated in Fig. 2-8, assuming an initial condition of the target axis aligned properly aligned, the off axis angle $\theta$ oscillate between zero and twice the precession angle. Assuming that the maximum tumble rate is known, the required spin rate can be calculated with equations from reference 8 .

$$
\Omega_{3}=\frac{M}{I_{3}} \cos \phi \quad \text { and } \quad \Omega_{1}=\frac{M}{I_{1}} \sin \phi \quad \text { so } \Omega_{3}=\Omega_{1} \frac{I_{1}}{I_{3}} \cot \phi \text {, }
$$

where $I_{x}$ is the moment of inertia about the $x$ axis. Given $\Omega_{1}$ and $\theta_{\max }$ simply choose $\phi=\theta_{\max } / 2$ to determine the required spin rate $\Omega_{3}$. Suppose as above that the maximum acceptable angle $\theta_{\max }$ is 0.026 radians, the maximum 
tumble rate $\Omega_{1}$ is 2 radians/s (about 8 times the maximum no spin tumble rate for $100 \mathrm{~ms}$ coasting time), and $\mathrm{I}_{1}=\mathrm{I}_{3}$. Then choose the precession angle $\phi$ $=0.013$ radians $(=\theta / 2)$, and apply equation (2-14) giving

$$
\Omega_{3}=\Omega_{1} \frac{I_{1}}{I_{3}} \cot \phi=\frac{2 \text { radians }}{\mathrm{s}} \cot (0.013 \text { radians })=154 \text { radians } / \mathrm{s}
$$

Stresses resulting from this spin rate are quite small and negligible. The preceding analysis is applicable for rigid body precession. If the target has vibrations that couple with the spinning or tumbling motion, the tumbling may be more difficult to control.

\subsection{Reliability and Cost}

The plant capacity factor is the fraction of time that the plant is operating times the average fractional power at which the plant operates. It will be desirable to use IFE power plants for base load capacity and operate them at $100 \%$ power nearly all of the time because the fuel costs are low and capitol costs are high. In the case of $100 \%$ power operation, availability and capacity factor are equal. There is substantial economic incentive to have as high of a plant capacity factor as possible. Plant capacity factors 4 in the range of $75 \%$ to $85 \%$ are typically assumed for IFE power plants.

The cost of target injection systems are expected to be a very small fraction of the total cost of an IFE power plant. The actual cost will be dependent on the type of injector used and on the tracking and alignment methods employed. No thorough cost estimates are known to have been made. Several studies have assumed costs for target injection systems and some of these assumed costs are summarized here. 
The SAFIRE code assumes a value of $\$ 20$ million (1980 dollars) for the target delivery, tracking, and alignment systems. ${ }^{9}$ Zuckerman's model from the HIFSA study based his assumptions on the Westinghouse ICECAP code. ${ }^{10,11}$ The cost is taken to be 7.9 million (1985 dollars) for a $10 \mathrm{~Hz}$ gun and scales with the square root of the rep rate. It is based on a cryogenic aluminum sabot accelerated to $300 \mathrm{~m} / \mathrm{s}$ in a tube using a linear synchronous motor with laser beam tracking. The Prometheus design study estimated target injection and tracking costs of $\$ 3.44$ million (1991 dollars) for $5.65 \mathrm{~Hz}$ and $\$ 2.74$ million (1991 dollars) for $3.5 \mathrm{~Hz}$ injection. 5 The OSIRIS AND SOMBRERO design studies assumed \$5 million (1991 dollars) as a conservative estimate of target injection and tracking costs. ${ }^{12}$ The HYLIFE-II study estimated the cost at 4.6 million for two injectors (1993 dollars). 13

There are fixed injection costs that are not dependent on rep rate and additional costs that scale approximately linearly with rep rate. As discussed in section 2.2.2, some injectors such as the gas gun can only accelerate one target at a time so that one injector would be good for a maximum of about 10 $\mathrm{Hz}$. If this threshold is exceeded, additional injectors would be required.

We have estimated the cost of materials for an experimental gas gun injection and tracking system at $\$ 31,000.14$ This price included components for the gas reservoir, gas firing valve, reloader, gun barrel, vacuum chamber, vacuum pumps, detection equipment, and timing and control equipment. This system was not designed for long life, or steady state operation with tritium filled cryogenic targets. Proper cost estimates should be made in the future.

Target injection and tracking is essential to the plant operation, but represents a small fraction of the capital cost. Maintenance of target injectors should be scheduled during shutdowns for repair of other plant components. 
Unscheduled shutdowns for target injection system repair should be minimized by providing redundant target injection systems. The actual reliability of target injection systems is unknown. However, reliability can be increased substantially with redundant systems. For example, two independent injectors which could each inject targets at the plant pulse rate, could be operated simultaneously at one half capacity to supply targets to the reaction chamber. If each could operate between scheduled shutdowns with $90 \%$ reliability, the combination would be $99 \%$ reliable. If one injector could be repaired while the plant is still operating, the injection system reliability could be even higher.

Based on the above discussion we summarize in table 2-2 the estimated cost of a target injection and tracking system with two injectors escalated to 1994 dollars.

Table 2-2

Target Injection System Cost Estimates

Study Name

SAFIRE

HIFSA

Prometheus

OSIRIS \& SOMBRERO

HYLIFE-II ( 2 injectors)
Cost \$ (study year)

20 million (1980)

7.9 million (1985)

3.4 million (1991)

5.0 million (1991)

4.6 million (1993)
Cost (1994 \$) 2 injectors

80 million

22 million

7.5 million

11 million

4.7 million 


\subsection{Target Shielding}

\subsubsection{Movable Shielding}

A shield can be moved to interpose itself between the target being exploded and the next target being loaded or being injected. The shield will reduce the heating and activation of the injector parts as well as limit the heating of the next target to perhaps 0.1 degree Kelvin. ${ }^{4}$ Shielding will allow more than one target to be in motion toward the chamber center at one time. Figure 2-9 is a schematic drawing of the capsules, chamber, and target neutron shield. A thickness $z_{\mathcal{S}}$ of shielding is placed between two capsules a distance $r$ apart. The shield is moved into place after capsule 1 enters the reaction chamber. The shield could possibly be a solid object exterior to the reaction chamber with active cooling or possibly a liquid that is injected inside the chamber. Alternatively (as described in the section 2.5.2), for slow moving targets, the curved trajectory due to gravity can put subsequent targets (i. e. capsule 2) out of the line of sight of neutrons.

\footnotetext{
${ }^{4}$ The value $0.1 \mathrm{~K}$ was chosen somewhat arbitrarily. The overall requirement is to keep the fuel frozen and attached to the inner surface of the capsule. The temperature of all the fuel must remain below about $18 \mathrm{~K}$ after heating from all sources. Target heating may also be limited by expansion stress to a few tenth's of a degree. If a larger total temperature change of several degrees is allowed, then a larger temperature change such as $1 \mathrm{~K}$ could be allowed here as well. Target heating is discussed further in section 2.6.
} 


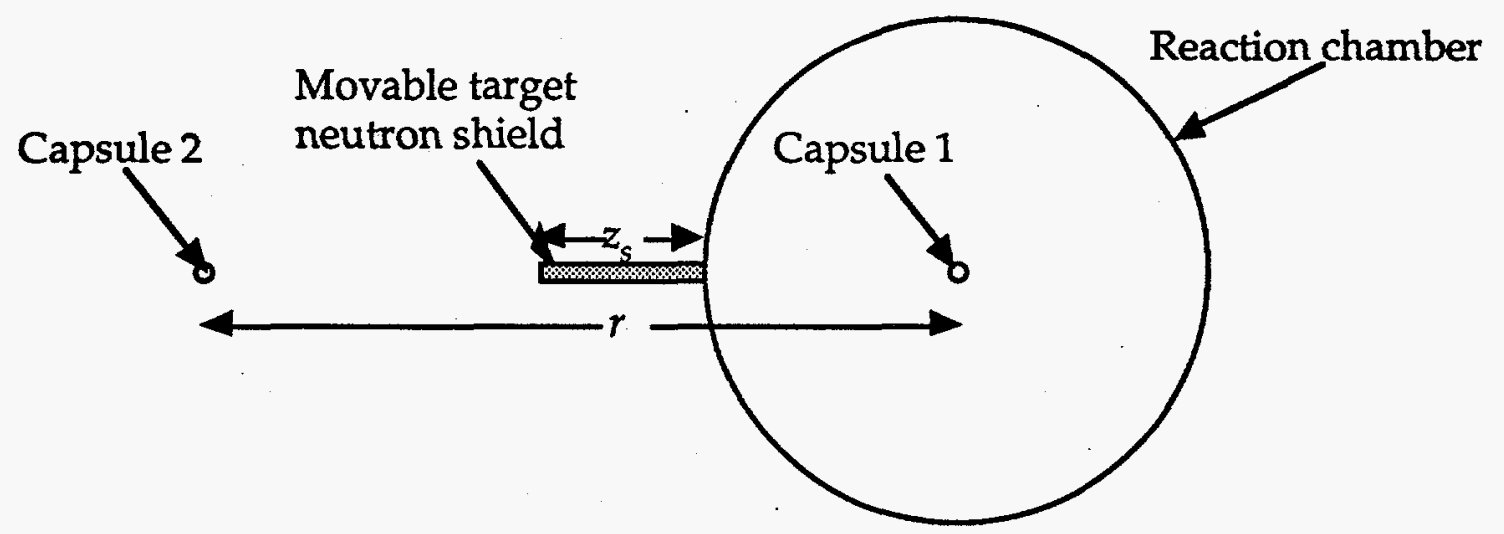

Figure 2-9. The neutrons from the micro-explosion of capsule 1 must be shielded to prevent excess heating of capsule 2 .

The unshielded capsule temperature rise $\Delta T$ can be obtained from

$$
E=m \int_{T_{1}}^{T_{2}} c(T) d T \approx m c \Delta T=\rho V c \Delta T
$$

for small temperature changes over which the specific heat capacity $c$ is nearly constant or where $c$ is understood to be a suitable average. Here, $E$ is the neutron energy locally absorbed, $\rho$ is the density of the DT fuel, and $V$ is the volume of the sample heated. The specific heat capacity of DT is a strong function of temperature, but at $16 \mathrm{~K}$ for example1, $c_{D T}=13.6 \frac{\text { Joules }}{\text { mole } \bullet \mathrm{K}}=2.7 \frac{\text { Joules }}{\mathrm{g} \cdot \mathrm{K}}$. (The specific heat capacities of DD and TT at $16 \mathrm{~K}$ are $8.1 \mathrm{~J} /$ Mole. $\mathrm{K}$ and $8.7 \mathrm{~J} / \mathrm{Mole} \cdot \mathrm{K}$ respectively for an average specific heat capacity of $11 \mathrm{~J} /$ Mole.K or 2.2 Joules/g.K). The density $\rho$ of solid DT is 1 $0.25 \mathrm{~g} / \mathrm{cm}^{3}\left(0.1 \mathrm{~g} / \mathrm{cm}^{3}\right.$ deuterium and $0.15 \mathrm{~g} / \mathrm{cm}^{3}$ tritium).

The energy deposited in the capsule is given by the number of neutrons that interact $\left(N_{I}\right)$ times the average energy deposited in the capsule $\left(E_{L}\right)$ from each interaction. The energy per unit volume absorbed in the fuel of an 
unshielded capsule is approximately the same as the energy that would be absorbed per unit volume by a spherical DT shell at radius $r$ from the neutron source and small thickness $\Delta r$.

$$
\begin{aligned}
\frac{E}{V} & =\frac{N_{I} E_{L}}{V} \\
& =\frac{N E_{L}}{A \Delta r}\left(1-e^{\frac{-\Delta r}{\lambda}}\right) \\
& \approx \frac{N E_{L}}{4 \pi r^{2} \Delta r} \frac{\Delta r}{\lambda}(\text { true for small } \Delta r) \\
& \approx \frac{Y E_{L}}{17.6 \mathrm{MeV}\left(1.6 \times 10^{-19} \mathrm{MJ} / \mathrm{MeV}\right) 4 \pi r^{2} \lambda} \\
& \approx \frac{Y E_{L}}{3.54 \times 10^{-17} \mathrm{MJ} r^{2} \lambda} .
\end{aligned}
$$

But $\frac{E}{V}=\rho c \Delta T$ so,

$$
\Delta T=\frac{Y E_{L}}{3.54 \times 10^{-17} \mathrm{MJ} r^{2} \lambda \rho c} .
$$

Where $N$ is the total number of fusion neutrons, $Y$ is the source energy yield (There is one neutron produced for each $17.6 \mathrm{MeV}$ of yield), and $\lambda$ is the total mean free path of neutrons in the DT.

Even though equation (2-18) is only valid for small temperature changes $\Delta T$ over which the specific heat capacity is nearly constant (or if $c$ is an average heat capacity), it can be used to determine the fraction of neutrons which must be shielded because the shielding will keep the temperature change small and $c$ nearly constant. The value of $E_{L}$ for $14 \mathrm{MeV}$ neutrons is ${ }^{15}$ 4.24 MeV in deuterium and $3.17 \mathrm{MeV}$ in tritium. The total mean free paths of 
$14 \mathrm{MeV}$ neutrons in deuterium and tritium are inversely proportional to the density and are given by ${ }^{15}$

$$
\begin{aligned}
& \lambda_{D}=\lambda_{D o} \frac{\rho_{D o}}{\rho}=2.49 \times 10^{4} \mathrm{~cm} \frac{1.67 \times 10^{-4} \mathrm{~g} / \mathrm{cm}^{3}}{0.1 \mathrm{~g} / \mathrm{cm}^{3}}=41.6 \mathrm{~cm} \\
& \lambda_{T}=\lambda_{T o} \frac{\rho_{T o}}{\rho}=2.06 \times 10^{4} \mathrm{~cm} \frac{2.51 \times 10^{-4} \mathrm{~g} / \mathrm{cm}^{3}}{0.15 \mathrm{~g} / \mathrm{cm}^{3}}=34.5 \mathrm{~cm} \\
& \lambda=\left(\lambda_{D}^{-1}+\lambda_{T}^{-1}\right)^{-1}=\left(\frac{1}{41.6 \mathrm{~cm}}+\frac{1}{34.5 \mathrm{~cm}}\right)^{-1}=18.9 \mathrm{~cm}
\end{aligned}
$$

where $\lambda_{\mathrm{Do}}$, and $\lambda_{\mathrm{To}}$ are the total mean free paths of $14 \mathrm{MeV}$ neutrons in standard densities of deuterium and tritium respectively. $\rho_{D o}$ and $\rho_{\text {To }}$ are the standard densities of deuterium and tritium. Since a neutron is more likely to interact with tritium than deuterium, the average energy per interaction is closer to that of tritium than deuterium (this is a small correction). This average interaction energy is given by

$$
\begin{aligned}
E_{L} & =\frac{\sum_{j} E_{L j} N_{I j}}{\sum_{j} N_{I j}}=\frac{\sum_{j} E_{L j} n_{j} \sigma_{j} \phi}{\sum_{j} n_{j} \sigma_{j} \phi}=\frac{\sum_{j} \frac{E_{L j}}{\lambda_{j}}}{\sum_{j} \frac{1}{\lambda_{j}}} \\
& =\frac{\frac{E_{L T}}{\lambda_{T}}+\frac{E_{L D}}{\lambda_{D}}}{\frac{1}{\lambda_{T}}+\frac{1}{\lambda_{D}}}=\frac{E_{L T} \lambda_{D}+E_{L D} \lambda_{T}}{\lambda_{D}+\lambda_{T}} \\
& =\frac{41.6 \mathrm{~cm}(3.17 \mathrm{MeV})+34.5 \mathrm{~cm}(4.24 \mathrm{MeV})}{41.6 \mathrm{~cm}+34.5 \mathrm{~cm}}=3.69 \mathrm{MeV}
\end{aligned}
$$

where $n_{j}$ is the number density of the $j$ th species and $\phi$ is the flux of $14 \mathrm{MeV}$ neutrons. 
Applying equations (2-18), (2-19), and (2-20) to a typical example of a $400 \mathrm{MJ}$ yield and a distance $r$ of $5 \mathrm{~m}$ we get

$$
\begin{aligned}
\Delta T & =\frac{Y E_{L}}{3.54 \times 10^{-17} \mathrm{MJr}^{2} \lambda \rho c} \\
& =\frac{400 \mathrm{MJ}(3.69 \mathrm{MeV})\left(1.6 \times 10^{-13} \mathrm{~J} / \mathrm{Mev}\right)}{3.54 \times 10^{-17} \mathrm{MJ}(500 \mathrm{~cm})^{2}(18.9 \mathrm{~cm})\left(0.25 \mathrm{~g} / \mathrm{cm}^{3}\right)(2.2 \mathrm{~J} / \mathrm{g} \bullet \mathrm{K})} \\
& =2.56 \mathrm{~K} .
\end{aligned}
$$

This calculation shows that with a relatively modest amount of shielding to reduce the neutron flux by a factor of roughly 25.6 , the neutron heating should not be excessive (e.g. $\Delta T=0.1 \mathrm{~K}$ for specific heat at $T=16 \mathrm{~K}$ ). This number scales linearly with the target yield and inversely with the square of the distance between the targets. The natural $\log$ of 25.6 is 3.24 . So, about 3.24 transport mean free paths of shielding is required to reduce the neutron flux adequately. For example, the transport mean free path for $14 \mathrm{MeV}$ neutrons in iron is $147.1 \mathrm{~cm}$. If iron were used for shielding, about $23 \mathrm{~cm}$ would be required to reduce neutron heating of the capsules.

This is a very simplified estimate of the shielding required to reduce capsule heating. An accurate calculation would not be one dimensional, it would consider neutrons reflected from structure that surrounds the capsule, it would consider the spectrum of neutrons leaving the shielding, it would consider heating from gamma's produced, and so would require a detailed knowledge of the particular power plant design.

However, the assumption of single energy (14 MeV) neutrons is conservative. The transport mean free path accounts for neutrons that are forward scattered through the shielding. The transport mean free path of iron tends to slowly decrease with decreasing neutron energy and is $5.5 \mathrm{~cm}$ at 1 
$\mathrm{MeV}$ (down from $7.1 \mathrm{~cm}$ at $14 \mathrm{MeV}$ ). The energy absorbed per distance traveled in the DT is proportional to the total cross section $\sigma$ times the energy deposited $E_{L} . \sigma E_{L}$ decreases in DT with decreasing neutron energy ${ }^{14}$. For example, $\sigma E_{L}$ in deuterium is $3.4 \mathrm{MeV}$-barns for $14 \mathrm{MeV}$ neutrons and 1.8 $\mathrm{MeV}$-barns for $1 \mathrm{MeV}$ neutrons and $\sigma \mathrm{E}_{\mathrm{L}}$ in tritium is $3.1 \mathrm{MeV}$-barns for 14 $\mathrm{MeV}$ neutrons and 1.0 MeV-barns for $1 \mathrm{MeV}$ neutrons. There will be some heating due to gamma's produced in the shielding from neutron interactions, but this should be minimal since penetration distance of these gamma's is much less than that of high energy neutrons. In addition, assuming that the rest of the reaction chamber has neutron shielding of similar neutron attenuating ability as the shielding in front of the target, direct shine and forward scattered neutrons greatly outnumber reflected neutrons ${ }^{16}$. So this calculation should give a rough upper bound of the amount of shielding required to control neutron heating of capsules.

Additional shielding may be required to protect the target injector or tracking optics from material damage. A movable shield would require cooling due to neutron heating and periodic replacement due to neutron induced damage.

\subsubsection{Target Trajectory Shielding}

The targets and target injector must be shielded from the neutrons, $x$ rays, and debris which are produced in each fusion event. It may be possible to use the gravitational arc in the target trajectories to keep the target injector and targets behind shielding. This section describes how to calculate whether targets and injectors can be adequately shielded from line of sight neutrons. Reflected and scattered neutrons are beyond the scope of this section.

Fig. 2-10 is a schematic drawing showing the geometry involved in making use of the gravitational arc. A coordinate system may be set up with a 
vertical $y$ axis and a horizontal $\mathrm{z}$ axis with origin at the muzzle end of the injector. The horizontal coasting distance from the origin to the center of the reaction chamber is $L_{c}$. The chamber radius is $r$. The target leaves the injector at time $t=0$. The target's horizontal speed is $v_{z}$. The target's horizontal position $z(t)$ is given by

$$
z(t)=v_{z} t
$$

The target's vertical position $y(z)$ is given by

$$
y=\frac{-g t^{2}}{2}=\frac{-g z^{2}}{2 v_{z}^{2}}
$$

where $g$ is the acceleration due to gravity. The vertical position of the target at the center of the chamber $y_{m}$ and at the edge of the chamber $y_{c}$ are given by

$$
y_{m}=\frac{-g L_{c}^{2}}{2 v_{z}^{2}} \text { and } y_{c}=\frac{-g\left(L_{c}-r\right)^{2}}{2 v_{z}^{2}}
$$

The line of sight passing from the target at the chamber center through the target opening at the chamber edge $y_{l}(z)$ is given by

$$
y_{l}=y_{m}+\left(y_{c}-y_{m}\right) \frac{\left(L_{c}-z\right)}{r}
$$




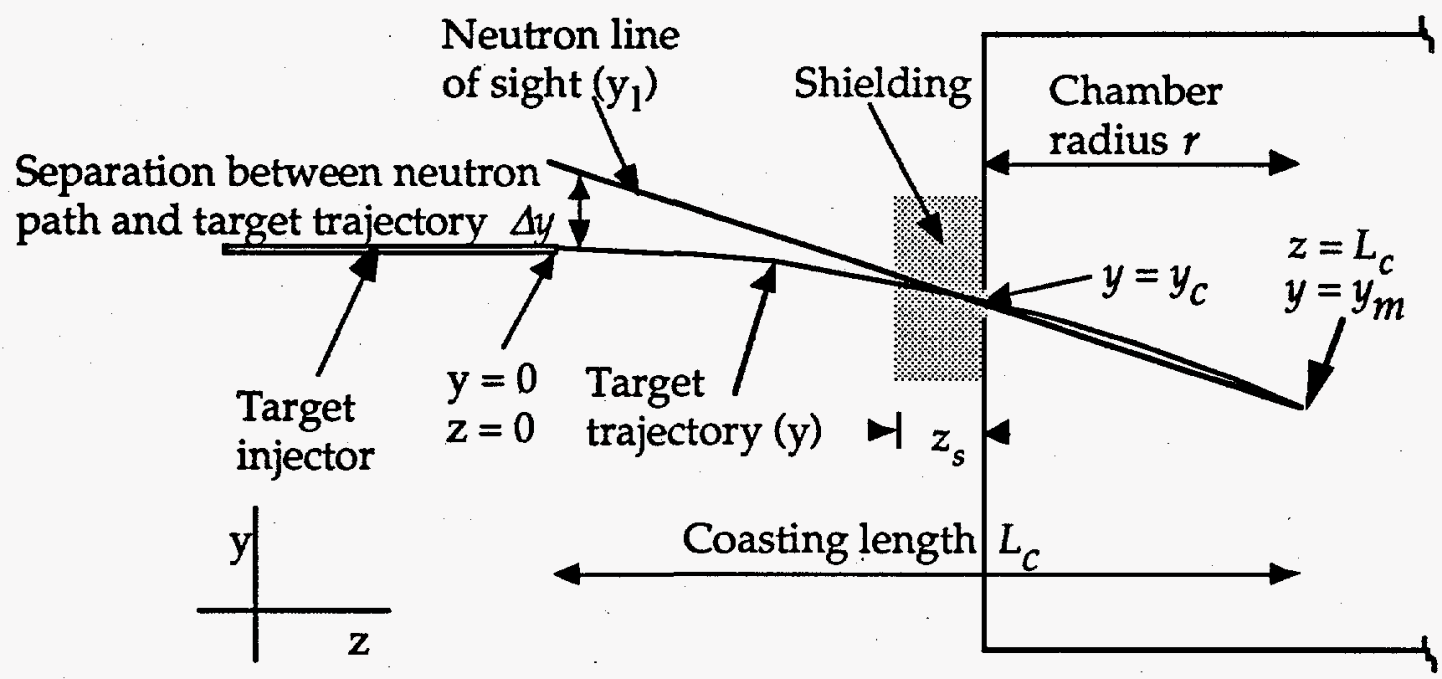

Figure 2-10. The arc in the target's trajectory due to gravity can aid in shielding the target injector and targets in flight toward the chamber.

The vertical distance $\Delta y$ between the line of sight $y_{l}(z)$ and the target trajectory $y(z)$ is simply

$$
\Delta y=y_{l}-y .
$$

$\Delta y(z)$ is the amount by which the center of the beam line of sight neutrons $y_{l}$ misses the center of the flight path of the subsequent target or the center of the target injector opening. The beam of line of sight neutrons is a cone that originates at the exploding target and passes through the target opening in the reaction chamber wall. The values of $y, y_{l}$, and $\Delta y$ are plotted in Fig. 2-11 for the example of an injection speed of $100 \mathrm{~m} / \mathrm{s}$, with coasting distance $7 \mathrm{~m}$ and a chamber radius of $3 \mathrm{~m}$. It shows that the maximum separation $\Delta y$ between the line of sight neutrons and the center of the target trajectory is about 14 $\mathrm{mm}$ and occurs at $\mathrm{z}=0$. 


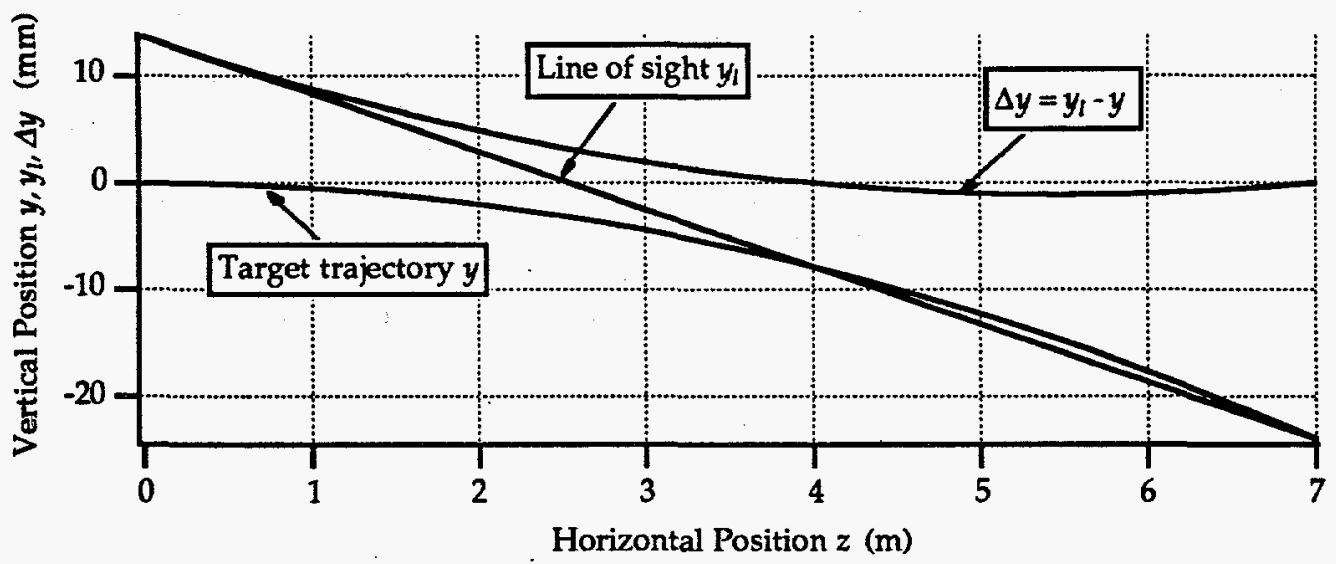

Figure 2-11. Target trajectory $y$, line of sight $y_{l}$, and distance between them $\Delta y$ vs. horizontal position $z$ for a target speed of $100 \mathrm{~m} / \mathrm{s}$, coasting distance $7 \mathrm{~m}$, and chamber radius of $3 \mathrm{~m}$.

$Y_{l}$ is the center of the beam of line of sight neutrons. The targets and target injector should be isolated from the outer edge of the beam. Scattered neutrons can originate from a variety of sources, but due to target compression, the source of neutrons is effectively a point source. The opening for the target to pass through the shielding and chamber wall has a radius $r_{s}$ that is larger than the target radius. As shown in Fig. 2-12, the neutrons can pass through unimpeded along $y l^{\prime}$ which is given by

$$
y_{l}^{\prime}=y_{m}+\left(y_{c}-r_{s}-y_{m}\right) \frac{\left(L_{c}-z\right)}{r}
$$

Line of sight neutrons below $y_{l}{ }^{\prime}$ will pass through some shielding. The equation for the line of sight neutrons that pass through a thickness $z_{s}$ of shielding is

$$
y_{l}{ }^{\prime \prime}=y_{m}+\left(y_{c}{ }^{\prime}-r_{s}-y_{m}\right) \frac{\left(L_{c}-z\right)}{r+z_{s}} \text { where } y_{c}{ }^{\prime}=\frac{-g\left(L_{c}-z_{s}-r\right)^{2}}{2 v_{z}^{2}}
$$




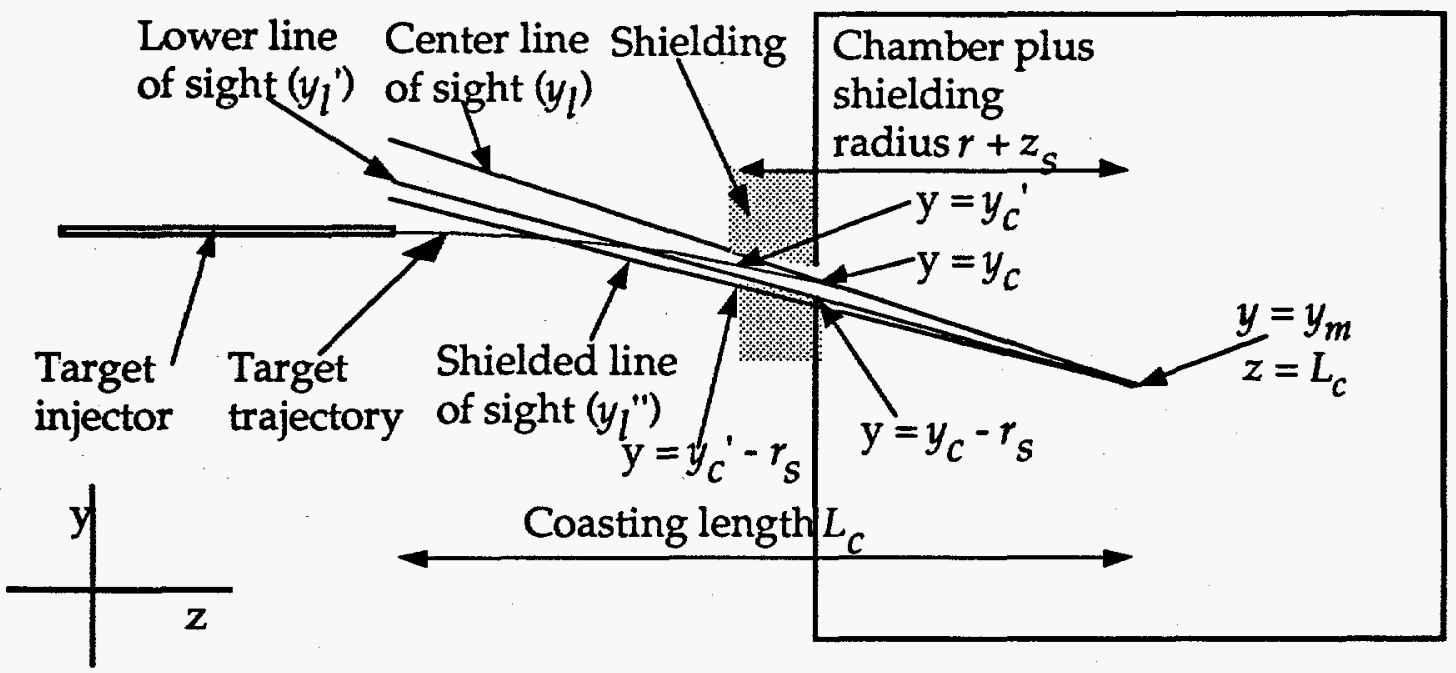

Figure 2-12. The gravitational arc in the targets trajectory may provide shielding, but the finite size of the hole in the shielding for target entry into the chamber must be considered.

To estimate whether the target trajectory will allow a thickness $z_{s}$ of shielding for the leading edge of the injector, one may calculate $y l^{\prime \prime}(z=0)$. If $y l^{\prime \prime}(z=0)$ is less than the radius of the target injector, part of the injector will not be fully shielded from line of sight neutrons. If $\Delta y^{\prime \prime}(z) \equiv y l^{\prime \prime}-y$ is less than the target radius, a target located at position $z$ will not be adequately shielded from line of sight neutrons. The value of $\Delta y^{\prime \prime}$ is very sensitive to target speed $v_{z}$ and to coasting length $L_{c}$. When greater than zero, $\Delta y^{\prime \prime}$ increases more rapidly than $\left(L_{c} / v_{z}\right)^{2}$. 


\section{Example:}

Suppose a target injection system had the following parameters.

\section{Injector radius}

horizontal speed

coasting length

desired shielding

chamber radius

shielding penetration radius

$$
r_{i}=20 \mathrm{~mm} \text {, }
$$$$
v=100 \mathrm{~m} / \mathrm{s} \text {, }
$$$$
L_{c}=7 \mathrm{~m},
$$$$
z_{s}=0.25 \mathrm{~m} \text {, }
$$$$
r=3 \mathrm{~m} \text {, and }
$$

$r_{s}=15 \mathrm{~mm}$.

(The injector radius is larger than target radius due to thickness of injector walls.)

Is the injector shielded from line of sight neutrons (i.e. is $\left.y l \mid(z=0)>r_{i}\right)$ ? Using equations (2-24) and (2-28) we find

$$
\begin{aligned}
y_{l}{ }^{\prime \prime}= & y_{m}+\left(y_{c}^{\prime}-r_{s}-y_{m}\right)\left(\frac{L_{c}-z}{r+z_{s}}\right) \\
& =\frac{-g L_{c}^{2}}{2 v_{z}^{2}}+\left(\frac{-g\left(L_{c}-z_{s}-r\right)^{2}}{2 v_{z}^{2}}-r_{s}+\frac{g L_{c}^{2}}{2 v_{z}^{2}}\right)\left(\frac{L_{c}-z}{r+z_{s}}\right) \\
& =\frac{-9.8 \mathrm{~m} / \mathrm{s}^{2}(7 \mathrm{~m})^{2}}{2(100 \mathrm{~m} / \mathrm{s})^{2}}+\left(\begin{array}{l}
\frac{-9.8 \mathrm{~m} / \mathrm{s}^{2}(7 \mathrm{~m}-0.25 \mathrm{~m}-3 \mathrm{~m})^{2}}{2(100 \mathrm{~m} / \mathrm{s})^{2}} \\
-0.015 \mathrm{~m}+\frac{9.8 \mathrm{~m} / \mathrm{s}^{2}(7 \mathrm{~m})^{2}}{2(100 \mathrm{~m} / \mathrm{s})^{2}}
\end{array}\right)\left(\frac{7 \mathrm{~m}-0 \mathrm{~m}}{3 \mathrm{~m}+0.25 \mathrm{~m}}\right) \\
& =-24 \mathrm{~mm}+(-6.9 \mathrm{~mm}-15 \mathrm{~mm}+24 \mathrm{~mm})(2.15) \\
& =-19.5 \mathrm{~mm}
\end{aligned}
$$

Since $y_{l} l^{\prime \prime}<r_{i}$, the injector is not adequately shielded. However, if coasting distance was increased to $16 \mathrm{~m}, y_{l}$ " would increase to $+23.5 \mathrm{~mm}$ which is 
greater than $r_{i}$ indicating that adequate shielding would be provided. Of course, less shielding would be needed with a larger coasting distance. Also, a longer coasting distance usually implies less target injection accuracy.

Some typical target injection scenarios would be shielded from line of sight neutrons, but most would not. Unfortunately, these simple calculations cannot assure adequate neutron shielding will be achieved. If these calculations show that adequate shielding might exist, further study would have to be performed to take scattered neutrons into account.

Moveable shielding is recommended to reduce heating, activation, and damage to targets and injector systems. Further study using neutron transport codes should be carried out.

\subsection{Target Heating}

\subsubsection{DT Fuel Temperature Limits}

DT fuel temperature for fusion energy is bounded from above and below by different factors. It is widely known that cryogenic targets with DT fuel frozen to the inner surface of the capsule will achieve higher gain than warm targets. Therefore, the temperature of the fuel must remain below the melting temperature of $19.7 \mathrm{~K}$. Additionally, for structural strength during the acceleration process, the fuel must remain a few degrees below the melting point. What is not as well known, is that the fuel temperature may be limited from below by required DT gas pressure inside a capsule. Through evaporation, this gas remains in near equilibrium with the inner surface of the fuel during the target injection process. The density of DT gas is a very strong function of temperature. ${ }^{1} \mathrm{DT}$ molecules are normally $25 \% \mathrm{D}_{2}, 25 \% \mathrm{~T}_{2}$, 
and 50\% DT. Table 2-3 shows how the saturated gas densities of these molecules varies with temperature from $12 \mathrm{~K}$ to $20 \mathrm{~K} .5$

Table 2-3. (After Ref. 1)

Estimated saturated vapor densities (moles $/ \mathrm{m}^{3}$ )

$\begin{array}{llll}\text { Temperature }(\mathrm{K}) & \mathrm{D}_{2} & \mathrm{DT} & \mathrm{T}_{2} \\ 12 & 1.0 & 0.47 & 0.23 \\ 14 & 6.3 & 3.4 & 1.9 \\ 16 & 26 & 16 & 9.6 \\ 18 & 79 & 52 & 34 \\ 20 & 182 & 136 & 97\end{array}$

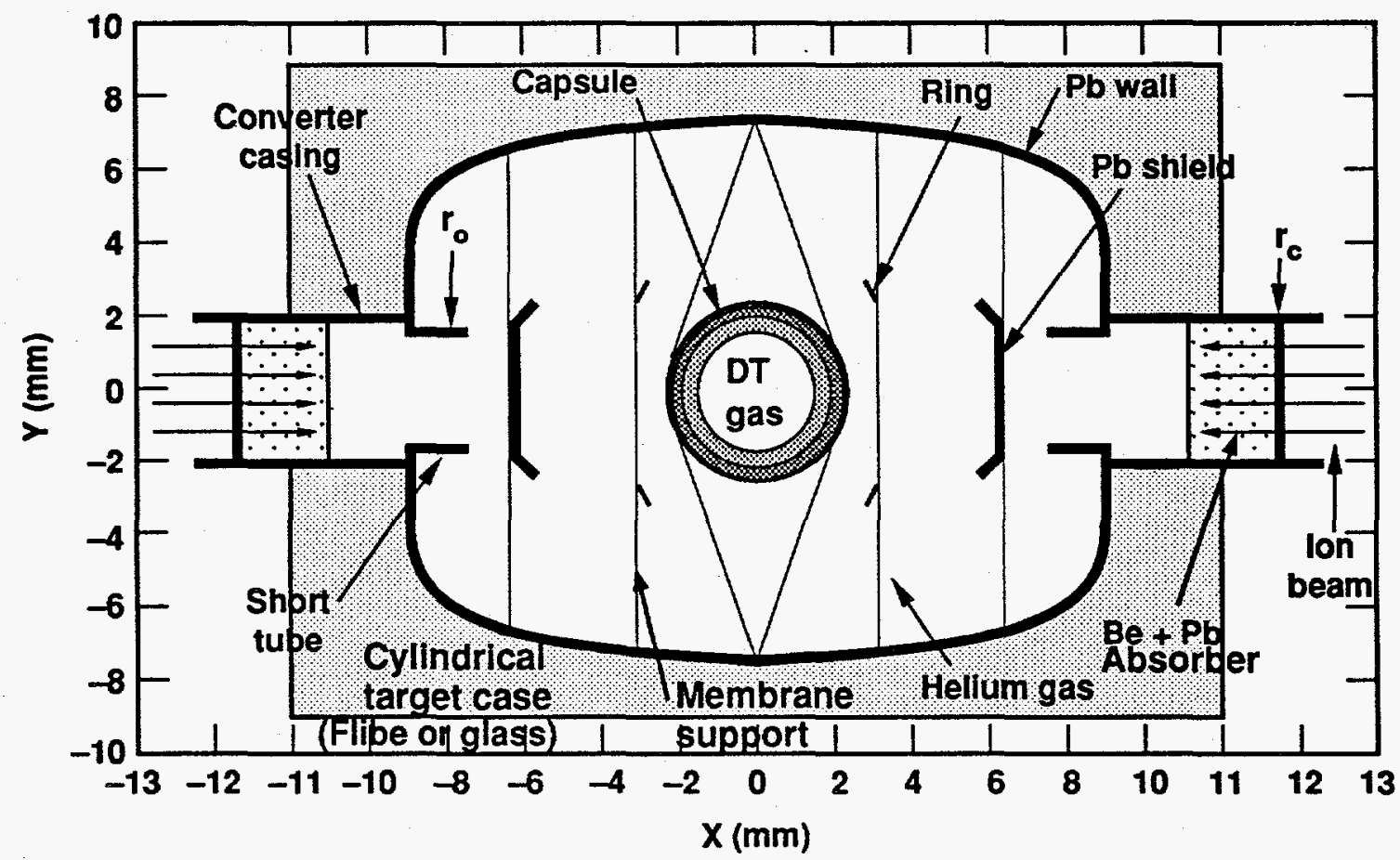

Figure 2-13. Inertial fusion energy target (designed by Darwin $\mathrm{Ho}^{17}$ ).

${ }^{5}$ Table 2-3 also shows that at each temperature the vapor density of $T_{2}$ is considerably less than the vapor density of $D_{2}$. This implies that the DT gas will actually be over $60 \%$ deuterium. This should be taken into account in target performance calculations. If the mixture results in substantially reduced gain, we recommend distilling pure DT with little $D_{2}$ or $T_{2}$ for use as fuel. 
Fig. 2-13 illustrates Darwin Ho's recent heavy-ion target design with membrane support of internal structure and a cylindrical external case made of Flibe or glass added. He used a DT gas density of $0.3 \mathrm{mg} / \mathrm{cm}^{3}\left(60 \mathrm{moles} / \mathrm{m}^{3}\right.$ for $5 \mathrm{~g} /$ mole DT). 17 This corresponds to a temperature of just over $18 \mathrm{~K}$. Since $18 \mathrm{~K}$ is near the freezing point of DT which is $19.7 \mathrm{~K}$, two concerns arise. The first concern is whether DT has adequate strength for injection with final temperature $18 \mathrm{~K}$. The second concern is, since the capsule is heated from the outside in during injection, will the outer fuel remain frozen. As will be shown, the news regarding both of the concerns is good, especially for indirect-drive targets.

Another potential limit on the target that is under active investigation at LLNL is fuel degradation due to stress associated with rapid fuel expansion. 18 This may limit target fuel heating to a few tenths of a degree. This effect could further reduce allowed target heating time or require that greater thermal insulation be designed into targets.

There is little data on the mechanical strength of DT at any temperature. However, ref. 19 gives Young's modulus and yield stress for $\mathrm{H}_{2}$ and $\mathrm{D}_{2}$. As shown in table 2-4, Young's modulus and yield strength increase with decreasing temperature. At a given temperature, they are higher for $D_{2}$ which has higher mass and a higher triple point. With no other data available, one could reasonably assume the strength of DT is higher than the strength of $D_{2}$ at the same temperature. Since the triple point of DT is one degree higher than $D_{2}$, one might expect the strength of DT to be similar to that of $D_{2}$ at one degree lower temperature. Chapter 4 shows that a yield stress of $0.05 \mathrm{MPa}$ (yield stress of $D_{2}$ at $16.4 \mathrm{~K}$ ) is sufficient for acceleration of $10,000 \mathrm{~m} / \mathrm{s}^{2}$. The following analysis assumes that during the acceleration process that the fuel temperature must remain below $17.4 \mathrm{~K}$ and that to maintain structural 
integrity during passage through the chamber, the outer fuel temperature must remain below $19.5 \mathrm{~K}$. The temperature of the inner fuel, which provides the DT gas pressure, will be slightly lower (Fig. 2-15 b) than the outer fuel since heat is being conducted from the hot reaction chamber environment.

Table 2-4. (After Ref. 19)

Yield stress and Young's modulus vs. temperature for $\mathrm{H}_{2}$ and $\mathrm{D}_{2}$

$\begin{array}{llll} & \text { Temperature }(\mathrm{K}) & \begin{array}{l}\text { Young's } \\ \text { Modulus } \\ \mathrm{E}(\mathrm{MPa})\end{array} & \begin{array}{l}\text { Yield } \\ \text { Stress } \\ (\mathrm{MPa})\end{array} \\ \mathrm{H}_{2}(\mathrm{TP}=13.9 \mathrm{~K}) & 8 & 20 & 0.18 \\ & 10 & 10 & 0.05 \\ & 12 & 5 & 0.025 \\ \mathrm{D}_{2}(\mathrm{TP}=18.7 \mathrm{~K}) & 11.6 & 90 & 0.16 \\ & 15.6 & 80 & 0.08 \\ & 16.4 & 40 & 0.05\end{array}$

An important question is, how cold can the fuel be without excessively decreasing target gain. To help answer this question, Darwin Ho calculated the effect on target gain of decreasing the gas pressure by $90 \%$ to $0.03 \mathrm{mg} / \mathrm{cm}^{3}$ ( $10 \%$ of his original $0.3 \mathrm{mg} / \mathrm{cm}^{3}$ or 6 moles $/ \mathrm{m}^{3}$ ). This density corresponds to a temperature of about $15 \mathrm{~K}$. With this lower density, the gain was $25 \%$ less. More data points would be very desirable, but the two data points available, indicate that targets can achieve high gain with inner fuel temperatures between $15 \mathrm{~K}$ and $18 \mathrm{~K}$ and perform much better near $18 \mathrm{~K}$.

As a base case, we'll make the conservative assumption that the fuel temperature can only rise $0.2 \mathrm{~K}$ from $17.3 \mathrm{~K}$ to $17.5 \mathrm{~K}$ during the injection 
process (excluding $0.1 \mathrm{~K}$ neutron heating from previous target neutron heating which is covered in section 2.5).

\subsubsection{Conduction Heat-transfer During the Injection Process}

A two-dimensional, finite-element, heat-transfer code (Topaz2d) was used to calculate target temperature rise. 20 We consider first the case of an indirect drive target followed by a brief discussion of direct drive target heating. We modeled the target using an axisymmetric wedge which is equivalent to 1-D spherical symmetry (Fig. 2-14). Using the conservative minimum dimensions of figure 2-14 with 1-D spherical symmetry is adequate to obtain an approximate heat conduction solution for the actual geometry shown in figure 2-13. Table 2-5 shows material property data points that were used for interpolation in the Topaz2d solution to this problem. $21,22,23,24,25$ The heat load of $50,000 \mathrm{~W} / \mathrm{m}^{2}$ corresponds to black body radiation at $970 \mathrm{~K}$ which is a typical power plant first wall temperature. For very low density gases, as will be found in most reaction chambers, the conductive and convective heating $q_{c}$ on the exterior of the capsule will be less than or about nkT $\bar{v}$. This would be approximately the amount of heat transferred if all chamber gas molecules that hit the target transferred an amount of energy equal to $\mathrm{kT}$. Using $n=$ $10^{13} / \mathrm{cm}^{3}, \mathrm{kT}=0.1 \mathrm{eV}$, and $\bar{v}=1000 \mathrm{~m} / \mathrm{s}$ (the average molecular speed for a molecular weight of about 20), gives $q_{c}=160 \mathrm{~W} / \mathrm{m}^{2}$. Therefore we see that the external conductive and convective heat loads should be negligible at densities below about $10^{14} / \mathrm{cm}^{3}$ but must be calculated at higher densities. The reference case initial target temperature is assumed to be $17.3 \mathrm{~K}$ with only 0.2 $\mathrm{K}$ temperature rise allowed. 
Table 2-5.

Approximate thermodynamic properties of target materials

\begin{tabular}{|c|c|c|c|c|c|}
\hline Material & $\begin{array}{c}\text { Density } \\
(\rho)\end{array}$ & Temperature & $\begin{array}{c}\text { Conductivity } \\
\text { (k) }\end{array}$ & $\begin{array}{l}\text { Specific } \\
\text { heat }(c)\end{array}$ & $\begin{array}{c}\text { Diffusivity } \\
(k / \rho c)\end{array}$ \\
\hline & $\mathrm{kg} / \mathrm{m}^{3}$ & & $\mathrm{~W} / \mathrm{m} \bullet \mathrm{K}$ & $\mathrm{J} / \mathrm{kgK}$ & $\mathrm{m}^{2} / \mathrm{s}$ \\
\hline DT & 250 & 8 & 1 & 240 & $1.6 \times 10^{-5}$ \\
\hline & & 12 & 0.9 & 1300 & $2.8 \times 10^{-6}$ \\
\hline & & 18 & 0.9 & 3500 & $1.0 \times 10^{-6}$ \\
\hline Plastic & 1000 & 8 & 0.06 & 20 & $3.0 \times 10^{-6}$ \\
\hline & & 20 & 0.09 & 100 & $9.0 \times 10^{-7}$ \\
\hline & & 50 & 0.11 & 240 & $4.6 \times 10^{-7}$ \\
\hline & & 100 & 0.13 & 450 & $2.8 \times 10^{-7}$ \\
\hline Helium & 1 & 8 & 0.0139 & 3100 & $4.5 \times 10^{-6}$ \\
\hline & & 20 & 0.0258 & 3100 & $8.3 \times 10^{-6}$ \\
\hline & & 50 & 0.0462 & 3100 & $1.5 \times 10^{-5}$ \\
\hline & & 80 & 0.0631 & 3100 & $2.0 \times 10^{-5}$ \\
\hline & & 150 & 0.095 & 3100 & $3.1 \times 10^{-5}$ \\
\hline Glass & 2000 & 8 & 0.1 & 2 & $2.5 \times 10^{-5}$ \\
\hline & & 20 & 0.15 & 25 & $3.0 \times 10^{-6}$ \\
\hline & & 50 & 0.25 & 180 & $7.0 \times 10^{-7}$ \\
\hline & & 80 & 0.45 & 300 & $7.5 \times 10^{-7}$ \\
\hline & & 200 & 0.8 & 1500 & $2.7 \times 10^{-7}$ \\
\hline
\end{tabular}

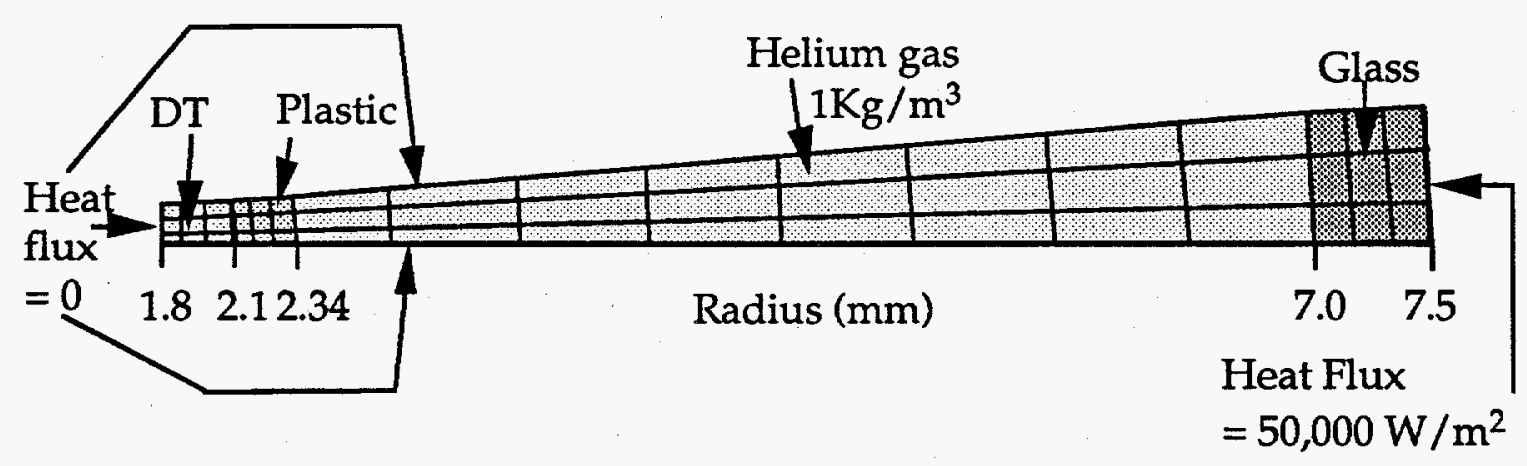

Figure 2-14. Model used to calculate heating of an indirect drive target. 
The 1-D spherical target model used for the computations is shown in Fig. 2-14. The DT and ablator are 0.3 and $0.24 \mathrm{~mm}$ thick respectively. Plastic was used for the ablator rather than beryllium since it is expected to be less costly to manufacture to required tolerances. The helium gas layer is $4.66 \mathrm{~mm}$ thick. The outer glass material was arbitrarily assumed to be $0.5 \mathrm{~mm}$ thick but could be made thicker if more thermal insulation is needed for the target. The helium gas density of $1 \mathrm{~kg} / \mathrm{m}^{3}$ is on the conservative low end of the expected 1 to $10 \mathrm{~kg} / \mathrm{m}^{3}$ range. ${ }^{25}$ With this gas density, the characteristic thermal diffusion time (thickness squared divided by thermal diffusivity) for the helium at $20 \mathrm{~K}$ is $2.6 \mathrm{~s}$. If a higher gas density is used the heat capacity would be greater and the characteristic time would be correspondingly longer, thereby further reducing the capsule heating rate.

Fig. 2-15 shows the temperature as a function of time at various positions within the target. The fuel temperature practically does not begin increasing for the first $150 \mathrm{~ms}$ and with an initial temperature of $17.3 \mathrm{~K}$ requires $350 \mathrm{~ms}$ for temperature to rise to $17.5 \mathrm{~K}$. Previous studies have shown much more rapid heating times ( $\mathrm{a}$ few tens of $\mathrm{ms}$ for many degrees temperature rise) for direct drive targets. ${ }^{21,26}$ These 1-D spherical calculations point out that indirect drive targets will heat much more slowly than direct drive. More refined 2-D or 3-D calculations with convection included should be done to verify these results. 


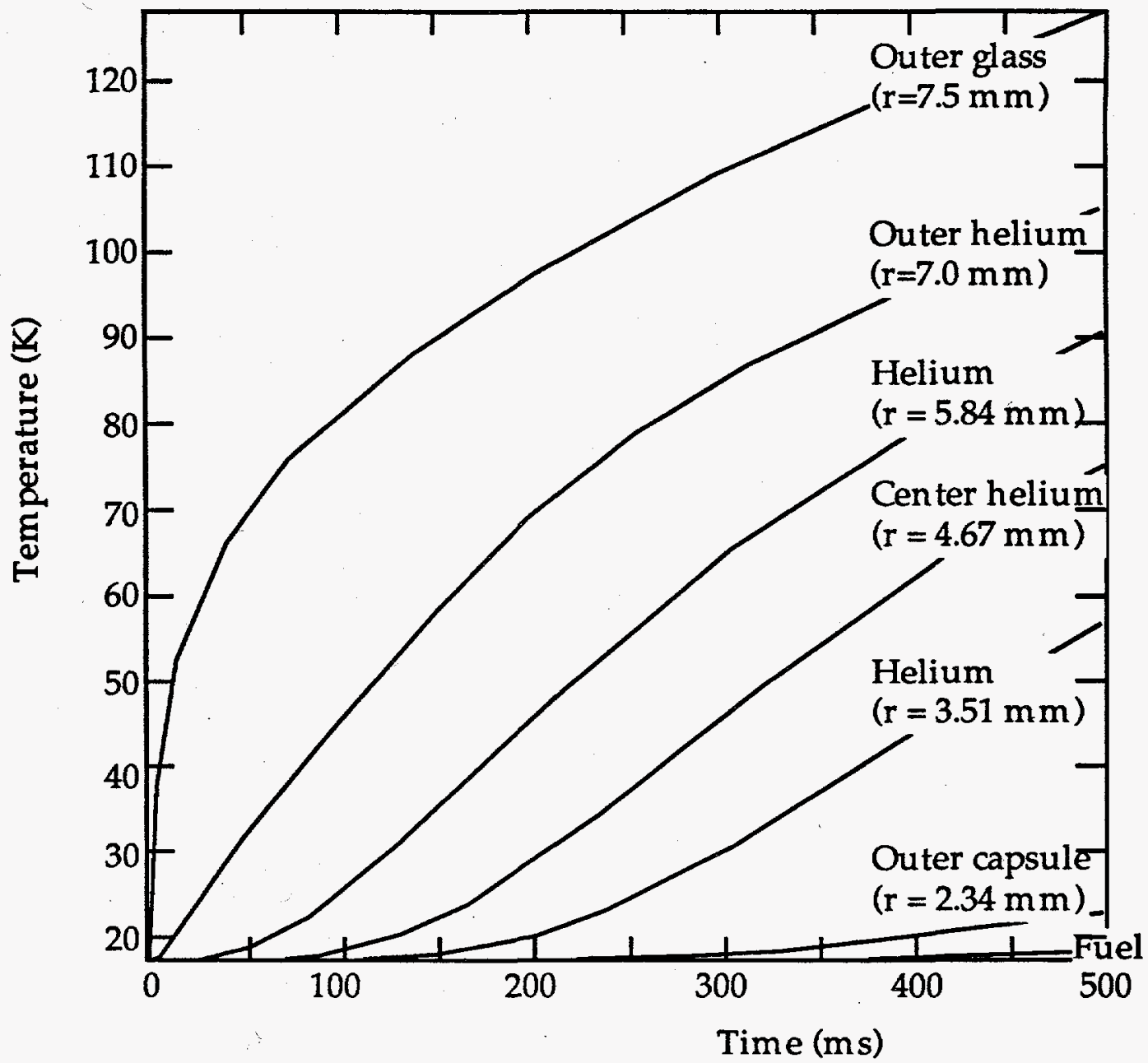

Figure 2-15a. Indirect-drive target temperature vs time. 


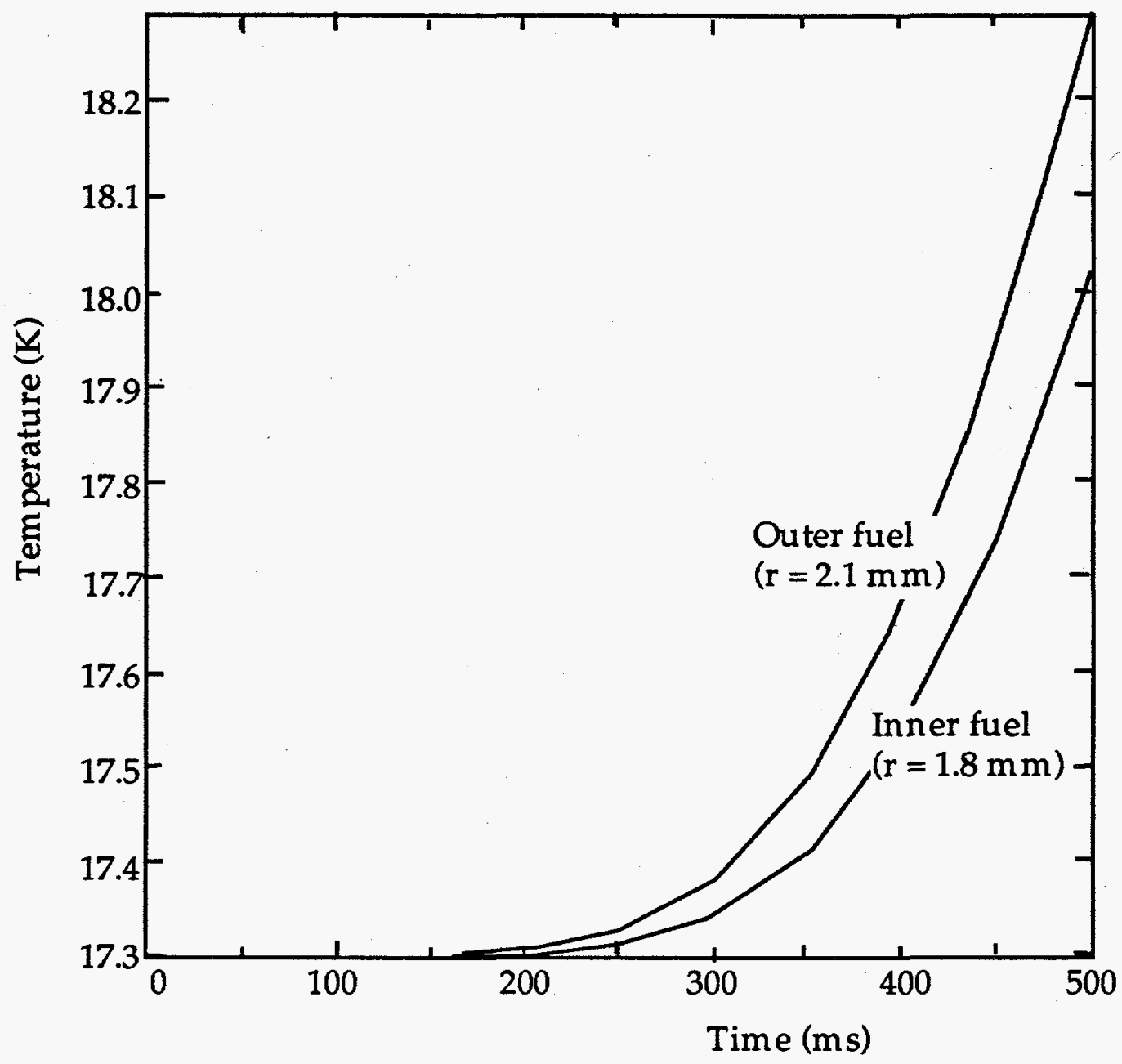

Figure 2-15b. Indirect-drive target fuel temperature vs time.

One aspect of target heating that was not yet considered, is the cooling of the inner fuel surface due to evaporation. If this were large enough cooling, it would help offset the undesirable heating. These capsules have a DT gas volume of $2.3 \times 10^{-8} \mathrm{~m}^{3}$. With density $0.3 \mathrm{~kg} / \mathrm{m}^{3}$ at $18 \mathrm{~K}$, the mass is $7 \mu \mathrm{g}$. The total enthalpy of DT gas at $18 \mathrm{~K}$ is $390 \mathrm{~J} /$ mole. ${ }^{26}$ The total enthalpy of DT solid at $18 \mathrm{~K}$ is $76 \mathrm{~J} /$ mole. $^{26}$ The energy of sublimation for DT at $18 \mathrm{~K}$ is (390-76) $\mathrm{J} / \mathrm{mole}$ or $63 \mathrm{~J} / \mathrm{g}$ for total energy of $0.44 \mathrm{~mJ}$. Making the conservative assumption that there was initially no gas in the capsule, if $0.44 \mathrm{~mJ}$ were removed from $3.7 \mathrm{mg}$ of solid DT in the capsule by sublimation of $7 \mu \mathrm{g}$ of DT, 
it would remove $0.6 \mathrm{~J} /$ mole of energy from the fuel and would cause a negligible temperature decrease of $0.03 \mathrm{~K}$ (i.e.

$\left.\frac{\mathrm{Q}}{m c}=\frac{0.44 \mathrm{~mJ}}{3.7 \mathrm{mg}(3.5 \mathrm{~J} / \mathrm{gK})}=0.03 \mathrm{~K}\right)$. The magnitude of capsule cooling due to sublimation would actually be much less, since most of the gas is already sublimated in the capsule when heating begins at $17.3 \mathrm{~K}$.

Another aspect not yet considered is radiative heating from the inner surface of the glass. It is assumed that the glass is opaque so that radiation from the outer surface inward is negligible. At a maximum temperature of 90 $\mathrm{K}$ (see Fig. 2-15 a), black body radiation is $5 \mathrm{~J} / \mathrm{m}^{2}$. If this heated the capsule with surface area $6.9 \times 10^{-5} \mathrm{~m}^{2}$ for $350 \mathrm{~ms}$, the heat absorbed would be $121 \mu \mathrm{J}$. This is clearly a negligible heating contribution.

Topaz2d calculations performed with the glass and helium removed from the target model of Fig. 2-14 are representative of direct drive target heating. The results are shown in Fig. 2-16. The capsule thickness for this reference case target is $0.24 \mathrm{~mm}$. To test the sensitivity of heating of the outer DT layer to capsule thickness, we kept the fuel layer the same and varied the thickness of the capsule from 0.2 to $0.3 \mathrm{~mm}$. The temperature of the outer fuel layer vs time is plotted for each of three capsule thicknesses on Fig. 2-17. As expected, the fuel heats much slower with a thicker capsule. For each of these capsules about 5 to $10 \mathrm{~ms}$ is required to heat the fuel from 17.3 to $17.5 \mathrm{~K} .18$ to $33 \mathrm{~ms}$ is required for the outer fuel temperature to reach $19.5 \mathrm{~K}$. At $100 \mathrm{~m} / \mathrm{s}, 30 \mathrm{~ms}$ is required for targets to transit to the center of a $3 \mathrm{~m}$ radius chamber.

If direct drive targets cannot tolerate a few degree temperature rise, we may have to inject the target at higher speed or possibly take action to reduce the heating rate. For example, a thin layer of material which reflects infrared radiation placed on the outer surface of the capsule could reduce the heating rate substantially. Typical metallic emissivities are in the range of 2 to $20 \% .24$ 
So by installing a thin layer of metal such as lithium on the capsule, we should be able to reduce the absorbed radiated heat by roughly an order of magnitude with this method. Fig. 2-18 shows the outer DT temperature of our reference indirect drive target (Fig. 2-14 without the helium or glass) vs time for four different surface heating fluxes starting at $17.3 \mathrm{~K}$. With the reference case heat flux of $5 \mathrm{~W} / \mathrm{cm}^{2}\left(50,000 \mathrm{~W} / \mathrm{m}^{2}\right)$, the outer DT temp rises to $17.5 \mathrm{~K}$ in $6 \mathrm{~ms}$ and passes through $19.5 \mathrm{~K}$ in $25 \mathrm{~ms}$. If this heating flux could be reduced by an order of magnitude to $0.5 \mathrm{~W} / \mathrm{cm}^{2}, 20 \mathrm{~ms}$ would be required to reach $17.5 \mathrm{~K}$ and the temperature after $40 \mathrm{~ms}$ would be just $18.0 \mathrm{~K}$. These slower heating rates would be of substantial benefit by allowing slower injection speeds. Further study is recommended on these coatings to determine their feasibility and the effect on target gain of adding this material. 


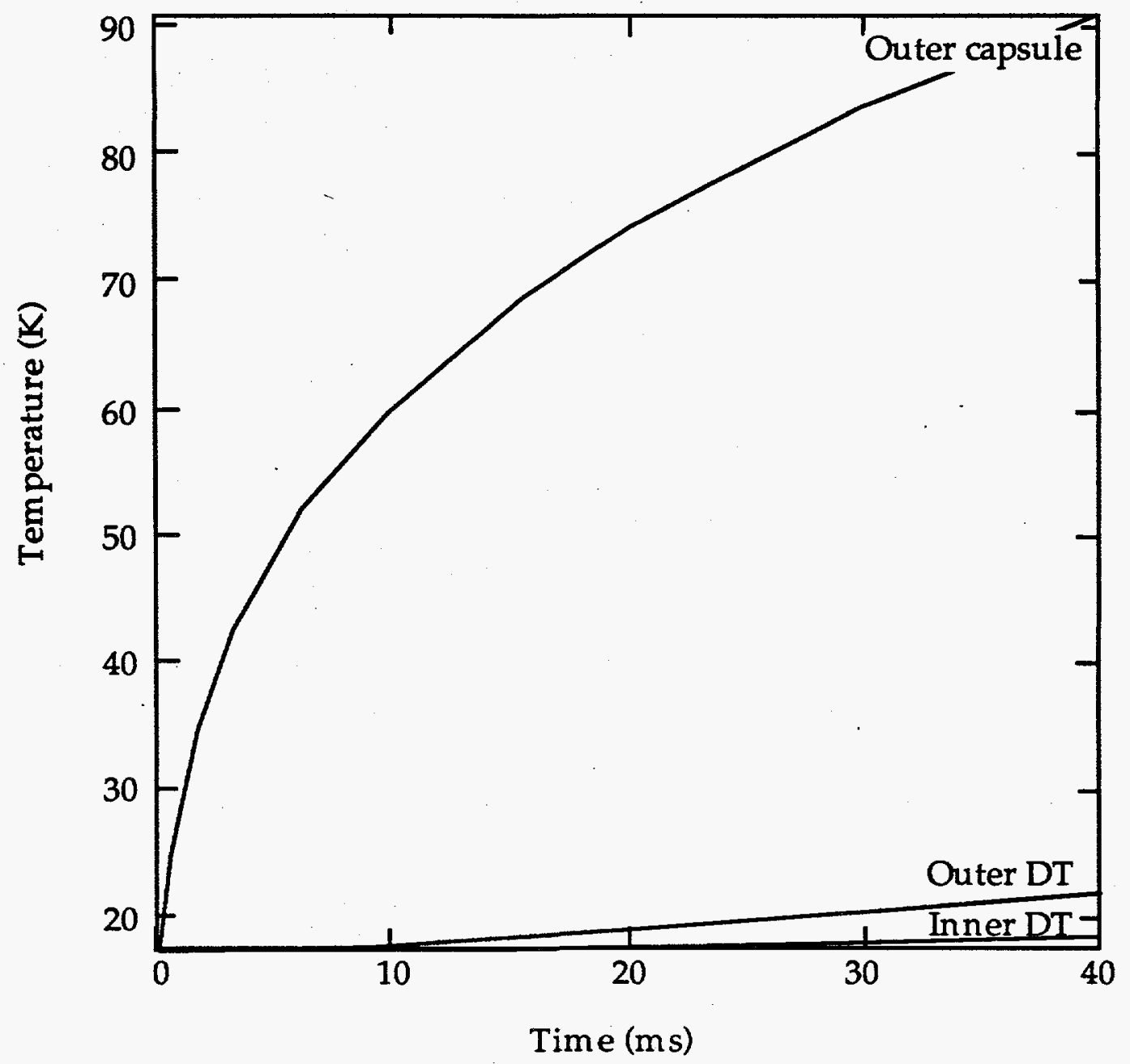

Figure 2-16. Direct-drive target temperatures vs time for reference case target with $5 \mathrm{~W} / \mathrm{cm}^{2}$ heat flux. 


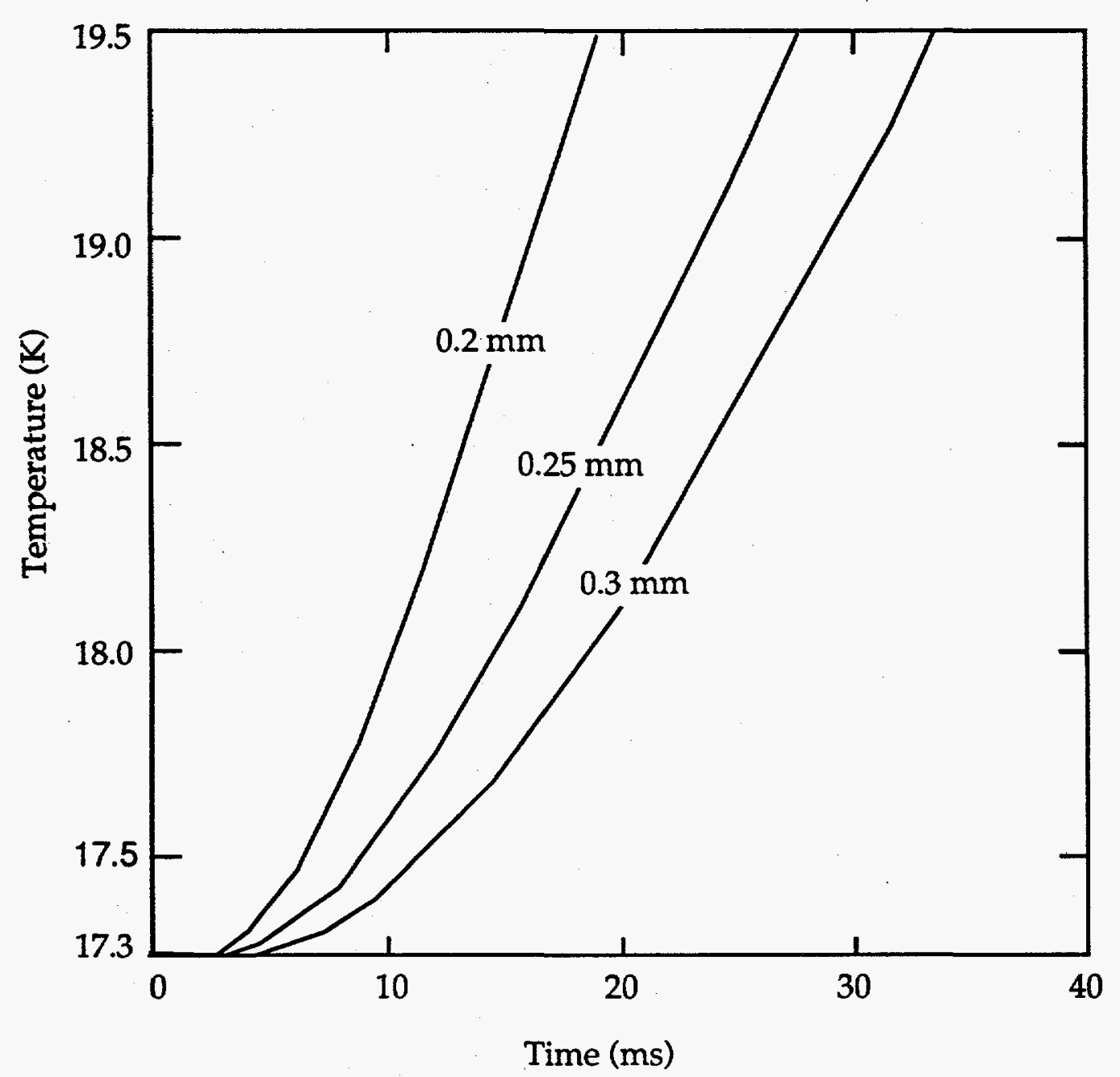

Figure 2-17. Direct-drive target outer DT temperature vs time for three different capsule thicknesses and $5 \mathrm{~W} / \mathrm{cm}^{2}$ heat flux. 


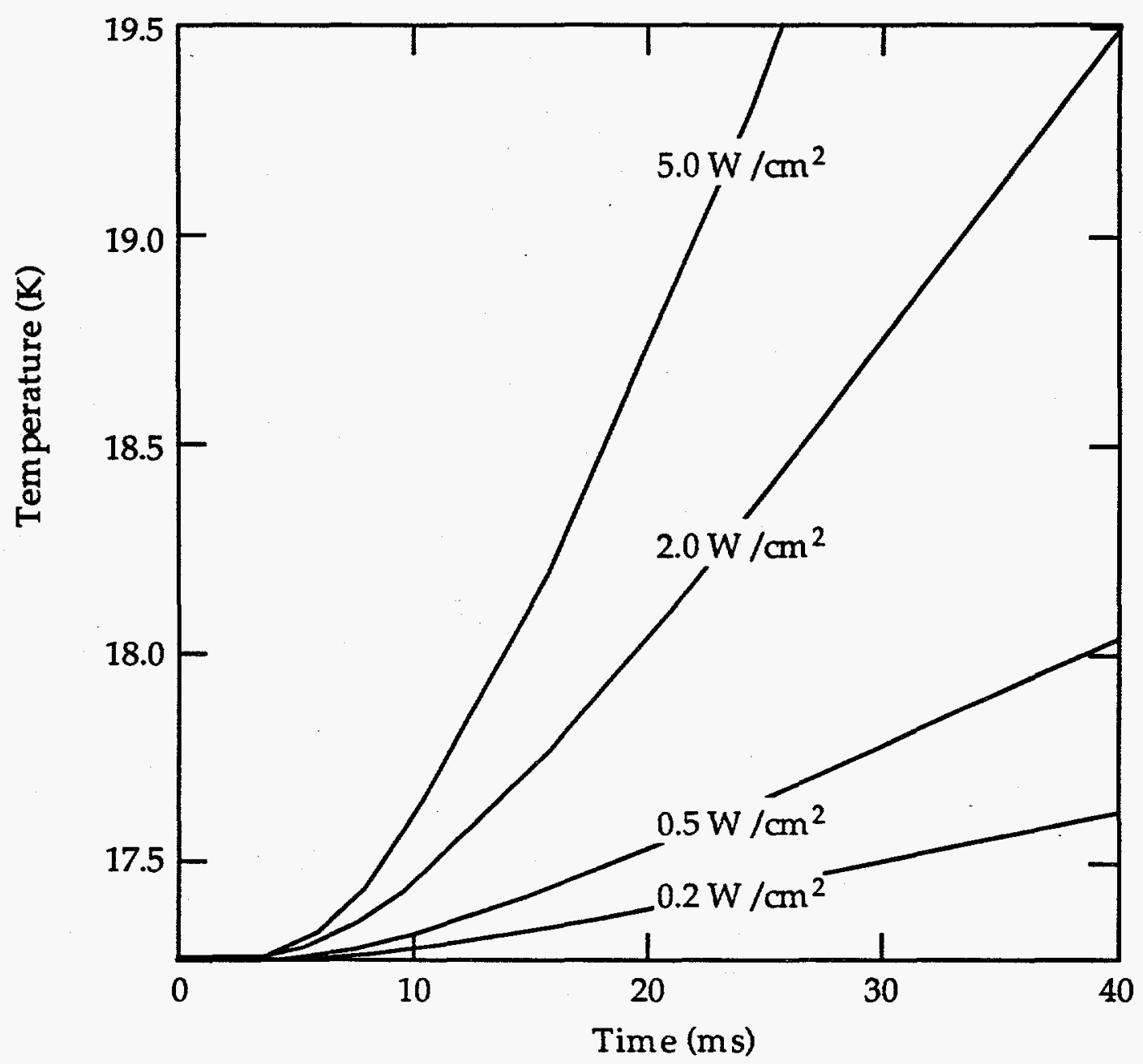

Figure 2-18. Direct-drive target outer DT temperature vs time for four different heat fluxes and $0.24 \mathrm{~mm}$ capsule thickness.

\subsubsection{Convective Heat-transfer During the Injection Process}

Another possible heat-transfer mechanism for indirect drive targets which has not yet been considered is convection heat-transfer via the helium within the target. As will be shown, convection heat flow in a target can be significant.

Fig. 2-19 illustrates the convective flow of gas involved in convective heat-transfer. Substantial time is required for convection flow to become established. A rough estimate of this time follows. First a substantial 
thickness of helium gas (about $1 \mathrm{~mm}$ ) must be heated several degrees so that it becomes substantially less dense than the colder helium. This initial heating time for a $10 \mathrm{~K}$ increase is estimated to be about $100 \mathrm{~ms}$ based on data like that of Fig. 2-15a. With a density difference established, gravity can force the cooler gas downward, forcing the warmer gas upward. If the cooler gas were twice as dense as the warmer gas with equal volumes of each, even neglecting viscosity, the system would be accelerated with less than one third g. The gas must then be accelerated over a distance of roughly the radius of the target, to convectively heat the capsule. With these assumptions, the required gas acceleration time $t_{a}$ is

$$
t_{a}=\sqrt{\frac{2 r}{g / 3}}=\sqrt{\frac{2(7.0 \mathrm{~mm})(3)}{\left(9.8 \mathrm{~m} / \mathrm{s}^{2}\right)}}=65 \mathrm{~ms} .
$$

Adding the helium heating time to the gas acceleration time gives a rough characteristic time for convective heat-transfer to become established of about $165 \mathrm{~ms}$. This rough time for convective heat transfer to become established is thought to be correct within about a factor of two.

Reference 27 reports the effect of steady state free convection heating within concentric cylinders and diatomic gases. This is the closest approximation I have found to transient spherical monatomic gas free convection. We proceed to use these results to obtain a rough approximation of the rate of convection heat-transfer. 


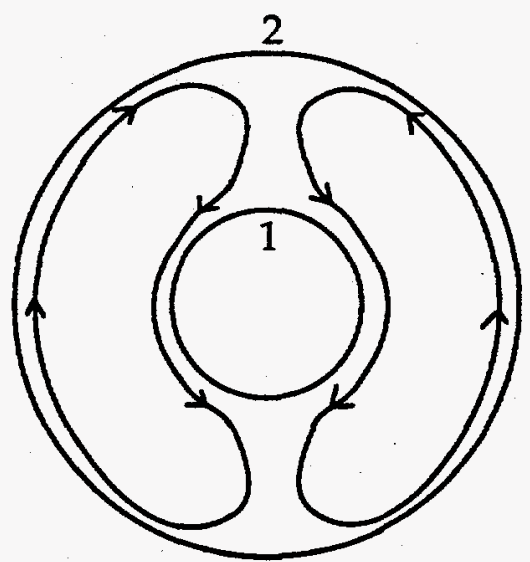

Figure 2-19. Free convection heat-transfer between two concentric cylinders or between two concentric spheres occurs due to fluid flow. This flow occurs due to density variations in the gas and is limited by viscosity.

The ratio of the total conduction and convection heat-transfer $k_{c}$ to the conduction heat-transfer $k$ is given by

$$
\frac{k_{c}}{k}=\phi\left[\left(N_{G r}\right)_{1}, \frac{D_{2}}{D_{1}}\right]
$$

where $D_{2}$ is the outside diameter, $D_{1}$ is the inside diameter, and $\left(N_{G r}\right)_{1}$ is the Grashof number using $D_{1}$ as a characteristic length. The actual form of the function $\phi$ must be experimentally determined. Grashof's number for an ideal gas is given by

$$
\left(N_{G r}\right)_{1}=\frac{g \Delta T D_{1}^{3} \rho^{2}}{T_{0} \mu^{2}}
$$

where $g$ is the acceleration due to gravity, $\Delta T$ is the temperature difference between the inner and outer cylinder, and $\mu$ is the dynamic viscosity. Inserting representative values into equation (2-31) gives 17,28 


$$
\begin{aligned}
\left(N_{G r}\right)_{1} & =\frac{g \Delta T D_{1}^{3} \rho^{2}}{T_{0} \mu^{2}} \\
& =\frac{9.8 \mathrm{~m} / \mathrm{s}^{2}(70 \mathrm{~K}-17 \mathrm{~K})\left(4.7 \times 10^{-3} \mathrm{~m}\right)^{3}\left(1 \mathrm{~kg} / \mathrm{m}^{3}\right)^{2}}{40 \mathrm{~K}\left(6 \times 10^{-6} \mathrm{~kg} / \mathrm{s} \cdot \mathrm{m}\right)^{2}} \\
& =37,400 .
\end{aligned}
$$

The diameter ratio is about $3.0(14 \mathrm{~mm} / 4.7 \mathrm{~mm})$. Applying these values to Fig. 25-9 of reference 27 gives $k_{c} / k=2.2$. This implies steady state heating between $70 \mathrm{~K}$ and $17 \mathrm{~K}$ about $120 \%$ greater than the steady state conduction heating between the same temperatures.

Although it may not be well justified, we looked at the target heating that results from increasing the helium conductivity by a factor 2.2. Thus we applied the steady state relationship between conduction heat transfer and effective heat transfer to the transient heat conduction problem. We used the target model shown in figure 2-14 and again used the Topaz2d heat transfer code. 20 The results are shown in figures $2-20 \mathrm{a}$ and $2-20 \mathrm{~b}$. We find that in this case the DT fuel heats from 17.3 to $17.5 \mathrm{~K}$ in about $200 \mathrm{~ms}$ rather than $350 \mathrm{~ms}$ found from heat conduction alone. 


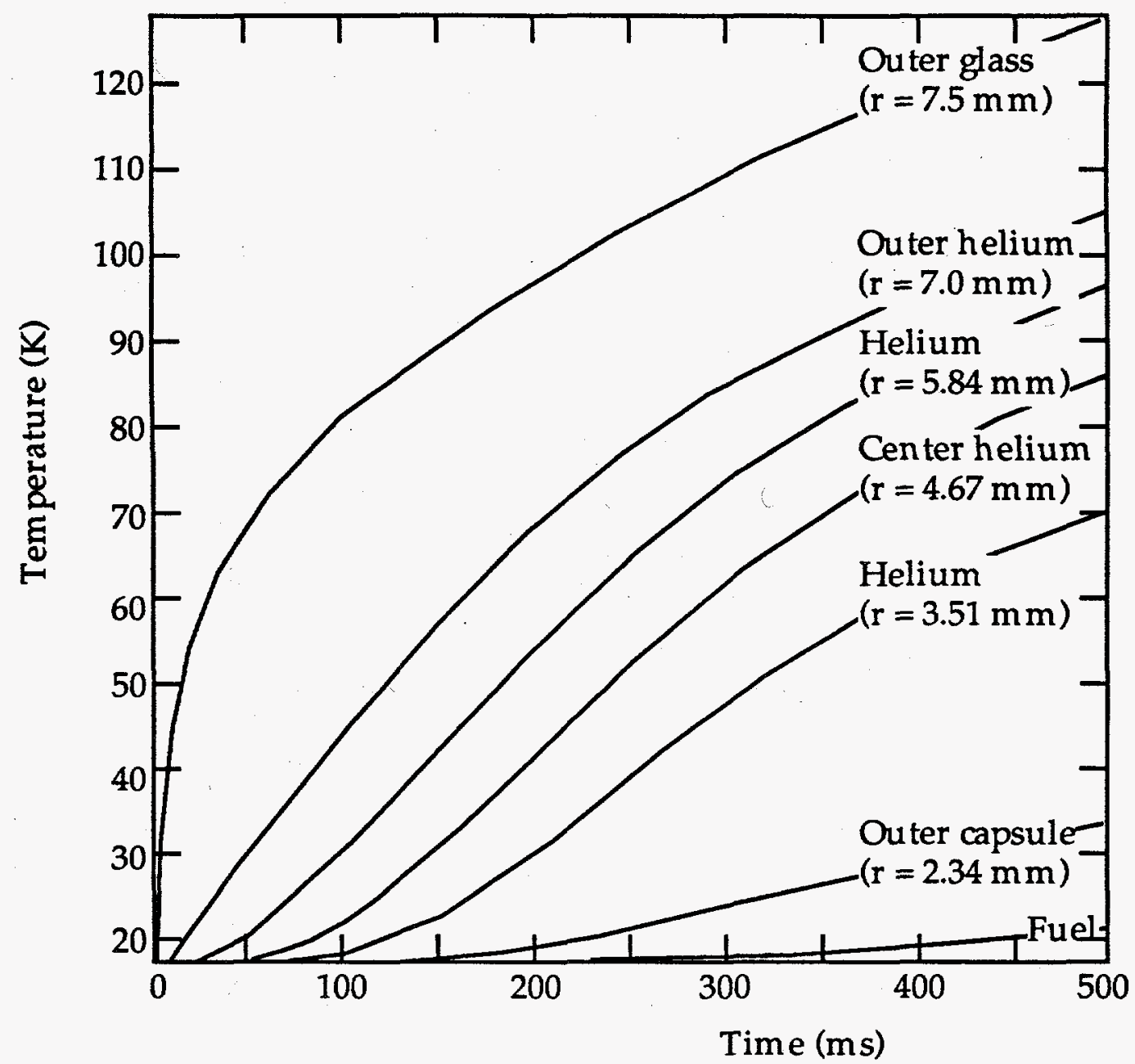

Figure 2-20a. Indirect-drive target temperature vs time with convection and conduction heating. Applies a steady state effective conductivity to the transient heat conduction problem. 


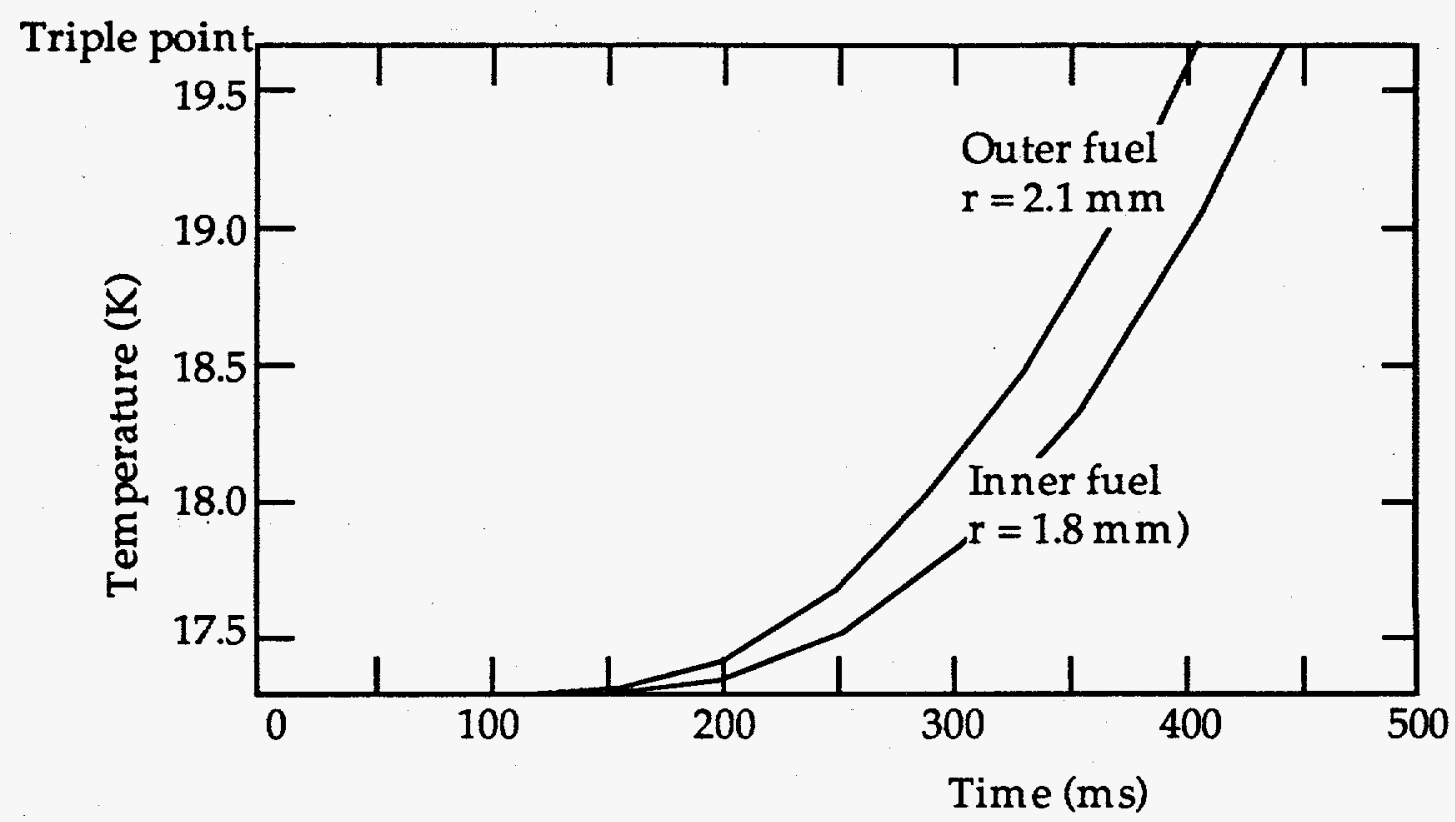

Figure 2-20b. Indirect-drive target fuel temperature vs time with convection and conduction heating. Applies a steady state effective conductivity to the transient heat conduction problem.

How close must the membranes be to effectively reduce convection heating? Convective heating between vertical surfaces will be reduced to less than 0.1 times conductive heating if Grashof's number $\left(\mathrm{N}_{\mathrm{Gr}}\right)_{\mathrm{L}}$ is less than about 2000 (the tests used diatomic gases). ${ }^{27}$ For an ideal gas,

$$
\left(\mathrm{N}_{\mathrm{Gr}}\right)_{\mathrm{L}}=\frac{g L^{3} \Delta T \rho^{2}}{T_{0} \mu^{2}}
$$

where $L$ is the distance between the surfaces. Solving for $L$ gives

$$
\begin{aligned}
L & =\sqrt[3]{\frac{2000 \mu^{2} T_{0}}{8 \Delta T \rho^{2}}} \\
& =\sqrt[3]{\frac{2000\left(6 \times 10^{-6} \mathrm{~kg} / \mathrm{m} \cdot \mathrm{s}\right)^{2}(40 \mathrm{~K})}{9.8 \mathrm{~m} / \mathrm{s}^{2}(70 \mathrm{~K}-15 \mathrm{~K})\left(1 \mathrm{~kg} / \mathrm{m}^{3}\right)}}=1.75 \mathrm{~mm} .
\end{aligned}
$$


So one way to adequately reduce convection heat-transfer is to divide the hohlraum into approximately $1.75 \mathrm{~mm}$ compartments.

Another possible method to disrupt convective heat flow is to spin the target. If the target undergoes a revolution, in a time period that is short compared to the time required for gravity to cause a cool dense gas to displace a lighter warm gas across the dimensions of a target, the convection heattransfer should be disrupted. If the target were to undergo a revolution in a time comparable or less than $65 \mathrm{~ms}$, normal convection flow would not be established.

However, if the target spin is started in the gun barrel, there may not be enough time for the gas in the target to come up to speed of rotation. We now estimate the time required for viscosity friction effects to accelerate the bulk of the gas to target rotation speed. Lamb ${ }^{29}$ reported a related case of one dimensional laminar fluid flow between two fluid sheets with infinite thickness in the $\mathrm{z}$ direction. The sheets in contact each other at the $\mathrm{z}=0$ position moving in opposite directions with initial speed $\pm U$. At time zero, the gas begins slowing due to viscosity. The speed of the gas $u$ is given by

$$
u=\frac{2 U}{\sqrt{\pi}} \int_{0}^{\theta} e^{-\theta^{2}} d \theta
$$

where in the upper limit,

$$
\theta=\frac{z}{\sqrt{4 v t}}=\frac{z}{\sqrt{4 \mu t / \rho}}
$$


where $v$ is the kinematic viscosity, $\mu$ is the viscosity, and $\rho$ is the density of the material. $u=U / 2$ when $\theta=0.48$. So to get a rough estimate of the for most the gas in the target to come up to rotational speed with the target we could use $z=2 \mathrm{~mm}$ (a typical distance of the gas from the target surfaces), helium gas density of $1 \mathrm{~kg} / \mathrm{m}^{3}$, helium viscosity of $35 \mu$ poise (for $20 \mathrm{~K}$ helium) 28 and solve for time $t$.

$$
t=\frac{z^{2} \rho}{4 \theta^{2} \mu}=\frac{\left(2 \times 10^{-3} \mathrm{~m}\right)^{2} 1 \mathrm{~kg} / \mathrm{m}^{3}}{4 \times(0.48)^{2} 35 \times 10^{-6} \mathrm{~g} / \mathrm{cm} \cdot \mathrm{s}} \frac{10 \mathrm{~g} \cdot \mathrm{m}}{1 \mathrm{~kg} \cdot \mathrm{cm}}=1.24 \mathrm{~s}
$$

So roughly a second is required for the gas rotation to come up to speed. Typical target injection times are an order of magnitude less. Additionally, while the gas is coming up to speed, vortices would likely occur which could increase convective heat transfer. Therefore if target spinning used either to reduce heat transfer, or to improve target directional stability as described in section 2.3.3, we recommend that membranes be placed in the target extending radially outward to force the gas to spin with the target. These membranes would also help reduce free convection heating. The hydrodynamic and design details are recommended for future work.

Indirect drive targets have the following advantage in controlling target heating. If target heating is calculated to be excessive, one may add more material to the outer hohlraum and substantially increase the targets characteristic thermal diffusion time.

In summary, for indirect drive targets, maintaining adequately low fuel temperature $(<17.4 \mathrm{~K})$ during acceleration and having sufficient DT gas density for high gain (corresponding to inner DT layer temperature of about $18 \mathrm{~K}$ ) are compatible objectives. We also assume that the fuel temperature rise 
must be limited to a few tenths of a degree to minimize stress from fuel thermal expansion. As our base case we take the temperature rise to be from 17.3 to $17.5 \mathrm{~K}$. In a typical reaction chamber with gas density less than $10^{14}$ per $\mathrm{cm}^{3}$, the majority of the external target heating is via radiation from the chamber walls. Within the target, convection and conduction heating dominate. A finite-element code was used to calculate conductive heating through a model one-dimensional spherical target model. Typical chamber temperature, target dimensions, and material properties were used in the calculations. The results for this model target follow. About $350 \mathrm{~ms}$ were required for the fuel temperature to rise from $17.3 \mathrm{~K}$ to $17.5 \mathrm{~K}$. With convection heating included we estimate this time is reduced to $200 \mathrm{~ms}$. If necessary, the effects of convection heating can be sufficiently reduced by separating the inner hohlraum into smaller compartments with membranes and by spinning the target.

Direct-drive targets heat much more rapidly than indirect drive targets which may require higher target injection speeds. A potential method of reducing target temperature rise is to put a thin layer of material external to the capsule which can reflect infrared radiation thereby reducing the external heat flux. This could increase the heating time substantially as shown in Fig. 2-18.

These computations have been preliminary in nature. Geometrically correct heat-transfer computations, including both convection and conduction, with more detailed material properties will be necessary to accurately predict target temperature rise. Detailed hydrodynamic calculations should be carried out especially if target spinning is required. However, sufficient time will be available to inject indirect-drive targets without 
excessive temperature rise even if additional material must be added to the outer hohlraum.

\subsection{Sabot Removal}

The target may have a sabot that is removed by centrifugal force as in Fig. 2-21. Previous studies ${ }^{3}$, have assumed that centrifugal force would cleanly separate a two piece sabot from a target capsule. However, calculations that follow show that a two piece sabot would probably exert a substantial and unpredictable impulse on the capsule as the pieces separate.

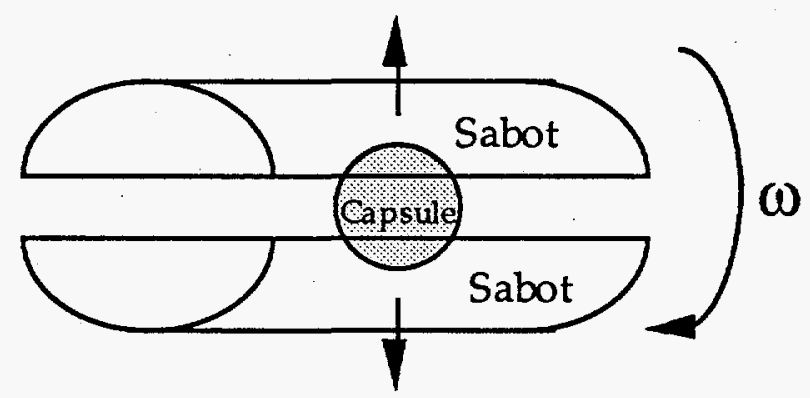

Figure 2-21. After the spinning target is accelerated, the sabot is removed from the capsule by centrifugal force.

As the sabot pieces separate from each other and from the capsule it is assumed that there is negligible adhesion (this assumption may not be justified). Once the sabot leaves the accelerator, if no external forces act on each piece, the linear and angular momentum of each piece are conserved. Fig. 2-22, concepts A and B show two cross-sections of a two piece cylindrical sabot surrounding a capsule. Concept $C$ shows a cross-section of a 4 piece sabot. The capsules are rotating clockwise in parts $A$ and $C$ and about the $z$ axis in part B. We wish to calculate the motion of the sabot pieces after release from the gun barrel for an $\mathrm{N}$ piece sabot. 


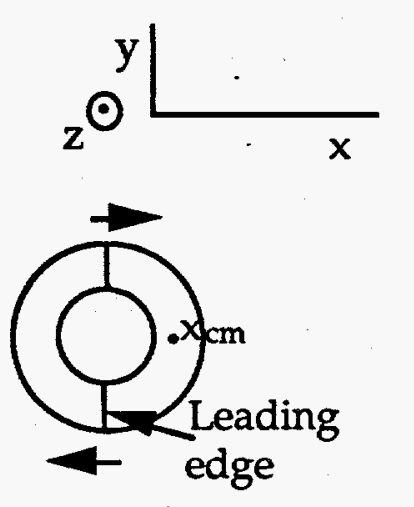

A End view

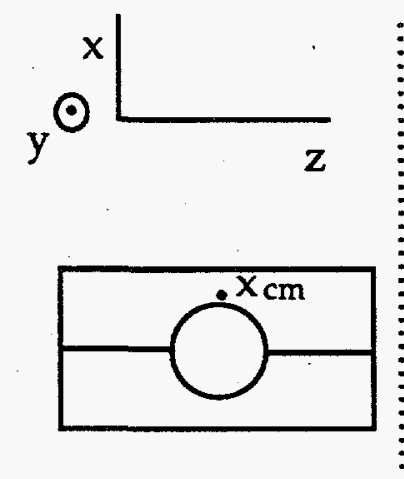

B side view

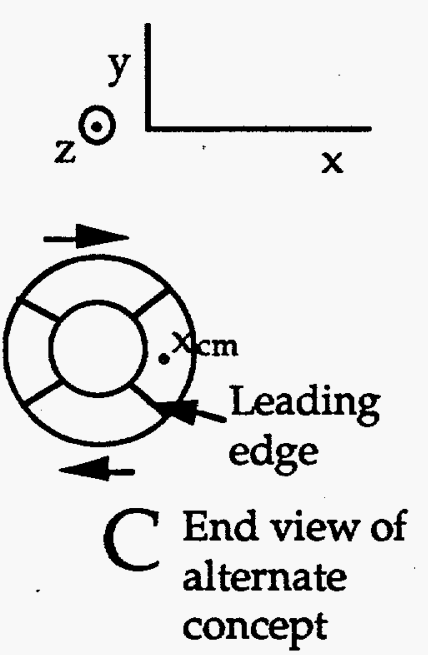

Figure 2-22. Concept A and B shows cross-sections of a two piece cylindrical sabot around a capsule. Concept $C$ shows a cross-section of a four piece sabot and capsule. After the target leaves the accelerator, the sabot pieces separate from the capsule. Linear and angular momentum are conserved.

To calculate the position of one sabot piece after release from the gun, two reference frames are useful. The unprimed reference frame has its origin at the center of mass of the sabot piece under study and rotates with the sabot piece. The primed reference frame is has its origin at the center of mass of the capsule and does not rotate. The prime coordinate system is illustrated in Fig. 2-23a. The relationship between the primed and unprimed coordinate system is illustrated in Fig. 2-23b. The coordinate systems are oriented in these frames such that the following relationships hold in transforming from one coordinate system to another when the target has rotated through an angle $\psi$. 


$$
\begin{gathered}
z^{\prime}=z \\
x^{\prime}=x \cos (\psi)+y \sin (\psi)+x_{c m} \\
y^{\prime}=-x \sin (\psi)+y \cos (\psi)-\psi x_{c m}
\end{gathered}
$$

and

where $x_{c m}$ is the $x^{\prime}$ component of the sabot piece's center of mass. (The $y^{\prime}$ coordinate of the center of mass is initially zero. The $x, y, z$, and $z^{\prime}$ coordinates of the center of mass are identically zero. ) The $\psi \mathrm{x}_{\mathrm{cm}}$ term comes from the relationship between the linear and angular momentum of the sabot piece and makes use of momentum conservation.

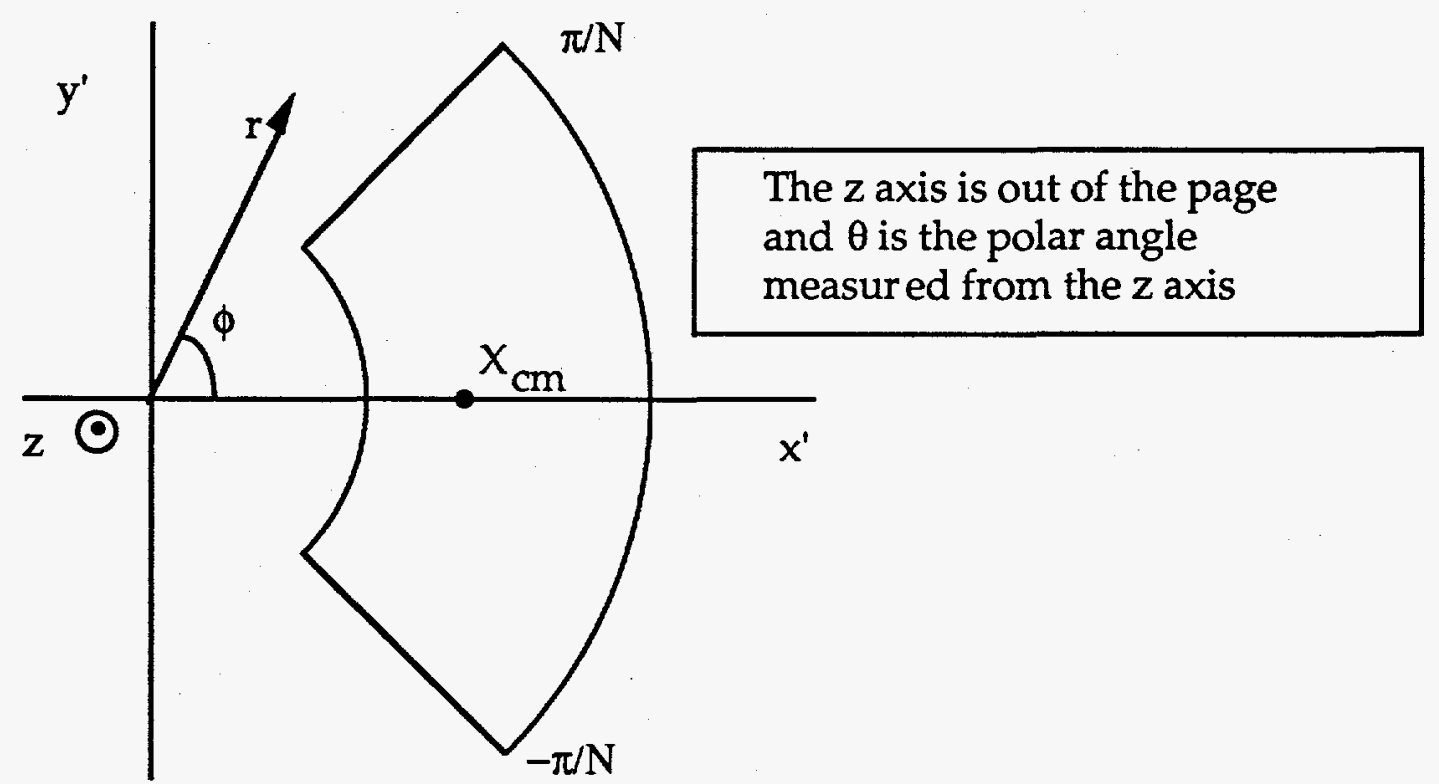

Figure 2-23a. Prime coordinate system at instant of sabot release from gun. 


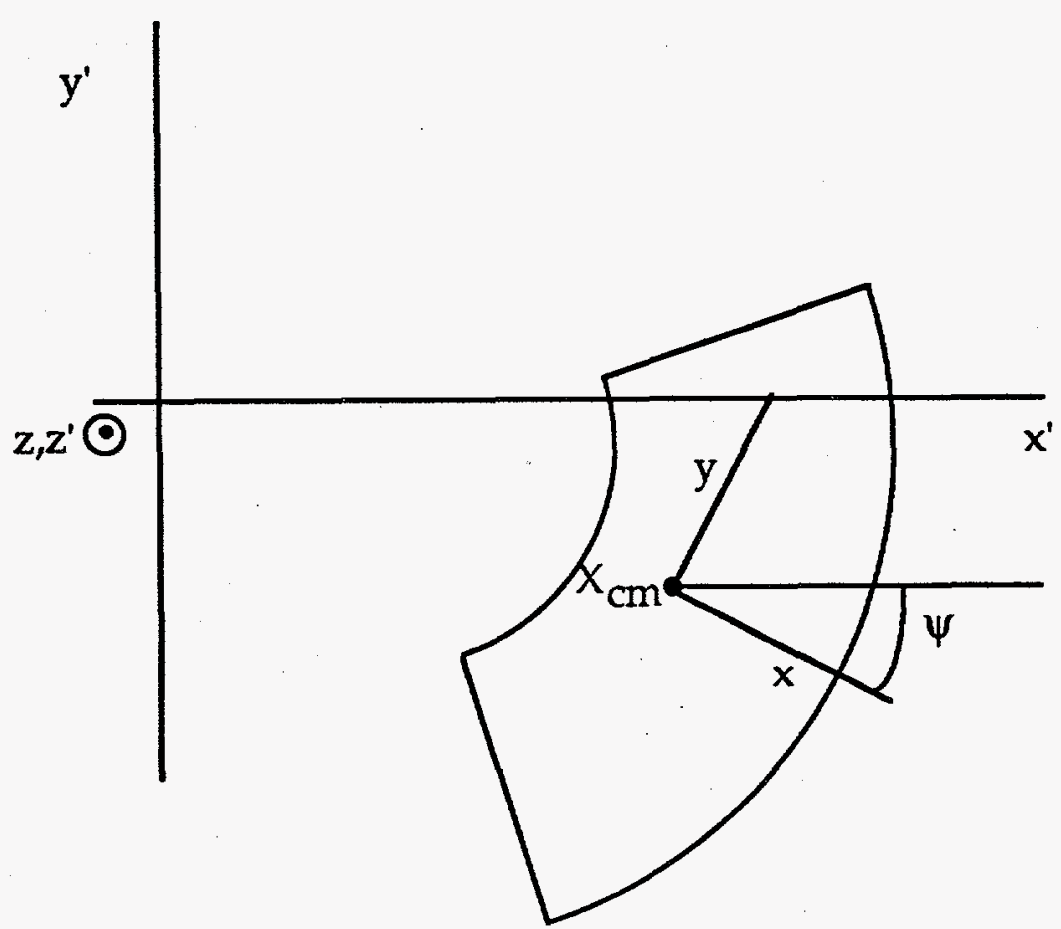

Figure 2-23b. Relationship between prime and unprime coordinate systems after sabot piece has rotated through an angle $\psi$.

To calculate $x_{\mathrm{cm}}$ of a single piece of an $N$ piece sabot, we assume the sabot is a cylinder with uniform density $\rho$, length $2 z_{0}$, and radius $r_{s}$. In the center of the sabot, is a spherical cavity of radius $r_{c}$ for the capsule. We first calculate the mass and center of mass of a solid piece of cylinder subtending an angle $2 \pi / N$.

$$
\begin{aligned}
& m_{\text {solid }}=\frac{\pi}{N} r_{s}^{2} 2 z_{o} \rho \\
& x_{\mathrm{cm}_{\text {solid }}}=\frac{\int_{0}^{r_{s}} \int_{\frac{-\pi}{N}}^{\frac{\pi}{N}} r \cos (\phi) r d \phi d r}{\frac{\pi}{N} r_{s}^{2}}=\frac{2 N}{3 \pi} \sin \left(\frac{\pi}{N}\right) r_{\mathrm{s}}
\end{aligned}
$$

The mass and center of mass of the hollowed out part of the sabot piece are 


$$
\begin{aligned}
m_{\text {hollow }} & =\frac{4 \pi}{3 N} \rho r_{c}^{3} \\
x_{c m_{\text {hollow }}} & =\int_{0}^{\pi} \int_{\frac{-\pi}{N}}^{\frac{\pi}{N}} \int_{0}^{r_{c}} r \sin \theta \cos \phi r^{2} \sin \theta d r d \phi d \theta \\
& =\frac{3 N}{16} \sin \left(\frac{\pi}{N}\right) r_{c}
\end{aligned}
$$

The net center of mass is then

$$
\begin{aligned}
x_{c m}= & \frac{\sum_{i} m_{i} x_{c m_{i}}}{\sum_{i} m_{i}} \\
= & \frac{\frac{\pi}{N} r_{s}^{2} 2 z_{o} \rho \frac{2 N}{3 \pi} \sin \left(\frac{\pi}{N}\right) r_{s}-\frac{4 \pi}{3 N} \rho r_{c}^{3} \frac{3 N}{16} \sin \frac{\pi}{N} r_{c}}{\frac{\pi}{N} r_{s}^{2} 2 z_{o} \rho-\frac{4 \pi}{3 N} \rho r_{c}^{3}} \\
= & \frac{\frac{4 N}{3} \sin \left(\frac{\pi}{N}\right) r_{s}^{3} z_{o}-\frac{N \pi}{4} \sin \left(\frac{\pi}{N}\right) r_{c}^{4}}{2 \pi r_{s}^{2} z_{o}-\frac{4 \pi}{3} r_{c}^{3}} .
\end{aligned}
$$

The $x$ and $y$ coordinates of the leading edge of the sabot piece are given by

$$
\begin{aligned}
& x=r \cos \left(\frac{\pi}{N}\right)-x_{c m} \text { and } \\
& y=-r \sin \left(\frac{\pi}{N}\right) \text { for } \\
& r_{c} \leq r \leq r_{s} .
\end{aligned}
$$

The previously defined transformation equations can be used to calculate the $x^{\prime}$ and $y^{\prime}$ positions of the leading edge of the sabot for various angles $\psi=$ $\omega t$. Here $\omega$ is the angular velocity of rotation and $t$ is the time after release from the gun barrel. If $x^{\prime 2}$ plus $y^{\prime 2}$ is less than $r_{c}{ }^{2}$, then the capsule and sabot 
are interfering with each others free motion. If such interference exists, the force applied on the capsule by the various sabot pieces would theoretically be symmetric and cancel. However, exact symmetry is not possible and a net non-repeatable impulse would be exerted on the capsule by the sabot pieces. This would adversely effect target injection accuracy.

Once the trajectory of the leading edge is calculated and plotted, a drawing program can be used to fill in the remainder of the sabot to help with visualization of the sabot trajectory. Figure 2-24a shows the trajectory of one piece of a two piece sabot with half length $z_{0}=r_{s}=2 r_{c}$. It is clear that the capsule would interfere with the sabot motion in this case and therefore target injection accuracy would probably be degraded. A similar result holds for all target-sabot size ratios. 


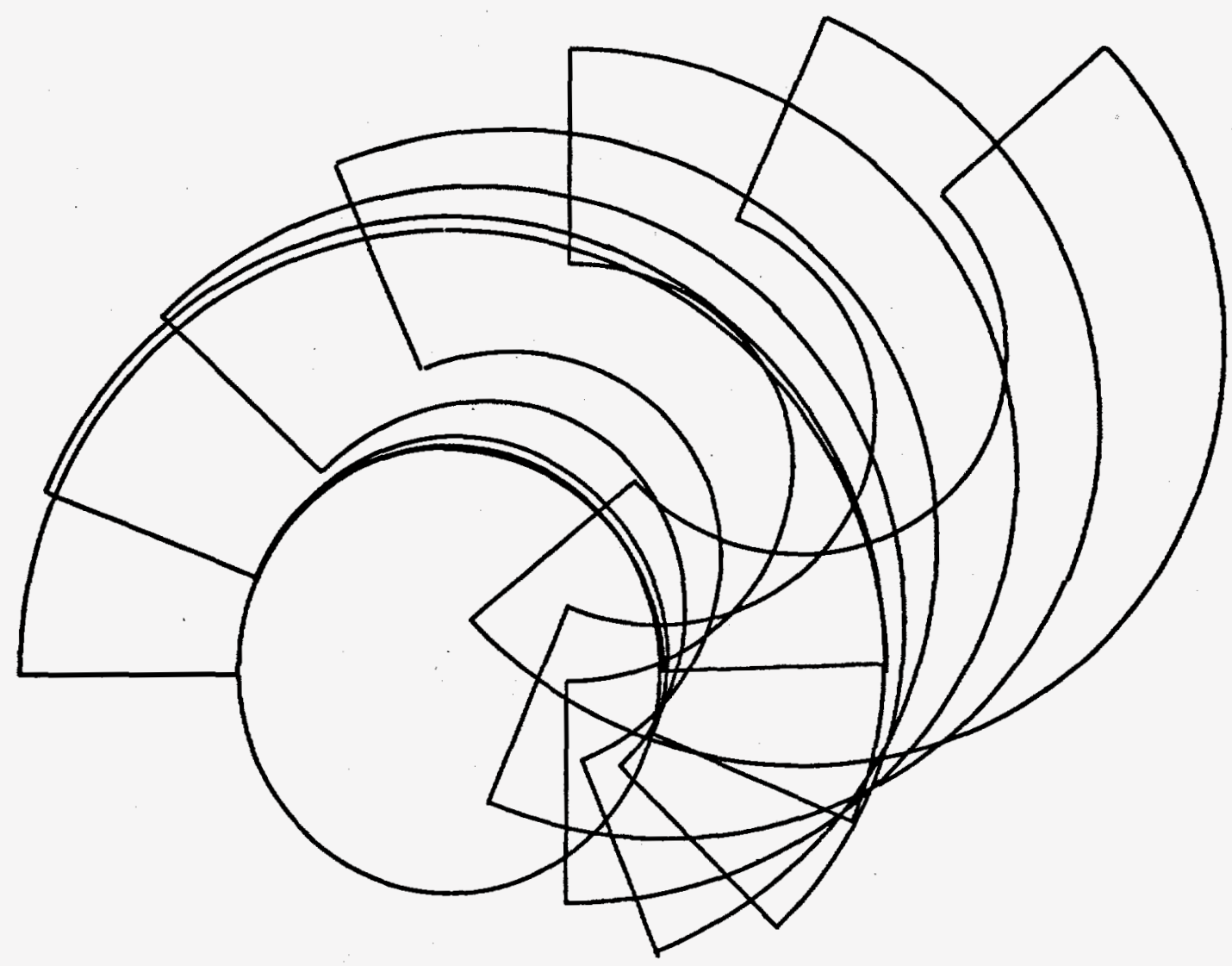

Figure 2-24a. Path that upper half of a two piece sabot would take when released from gun if not restrained by the capsule (superposition of many positions). The actual path would be modified by a force exerted from the capsule on the corner of the sabot.

This problem of interference between the capsule and sabot can be eliminated by dividing the sabot into more pieces. A three piece sabot will separate cleanly for smaller capsules $\left(r_{\mathfrak{c}} / r_{\mathfrak{s}}\right.$ less than about 0.7$)$, but not for larger capsules. A four piece sabot will separate cleanly for all capsule sizes. Figure 2-24b shows the path of a 4 piece sabot leaving the capsule for $z_{0}=r_{s}=$ $2 r_{c}$. The sabot separates cleanly without having to exert a force on the capsule. The figure shows a slice of the sabot at the $z=0$ plane. The dashed lines on the left side of figure $2-24 \mathrm{~b}$ indicate the shape of the sabot pieces at the ends of 
the sabot where material is not removed for the capsule. Once the entire sabot is out beyond the capsule radius, a stationary stripping device could redirect the sabot pieces allowing the capsule to proceed alone. As can be seen from Fig. 2-24b, the sabot takes about a quarter turn to be completely free of the capsule. If the barrel rifling pitch was one turn per meter, a quarter meter distance would be required to clear the sabot from the capsule.

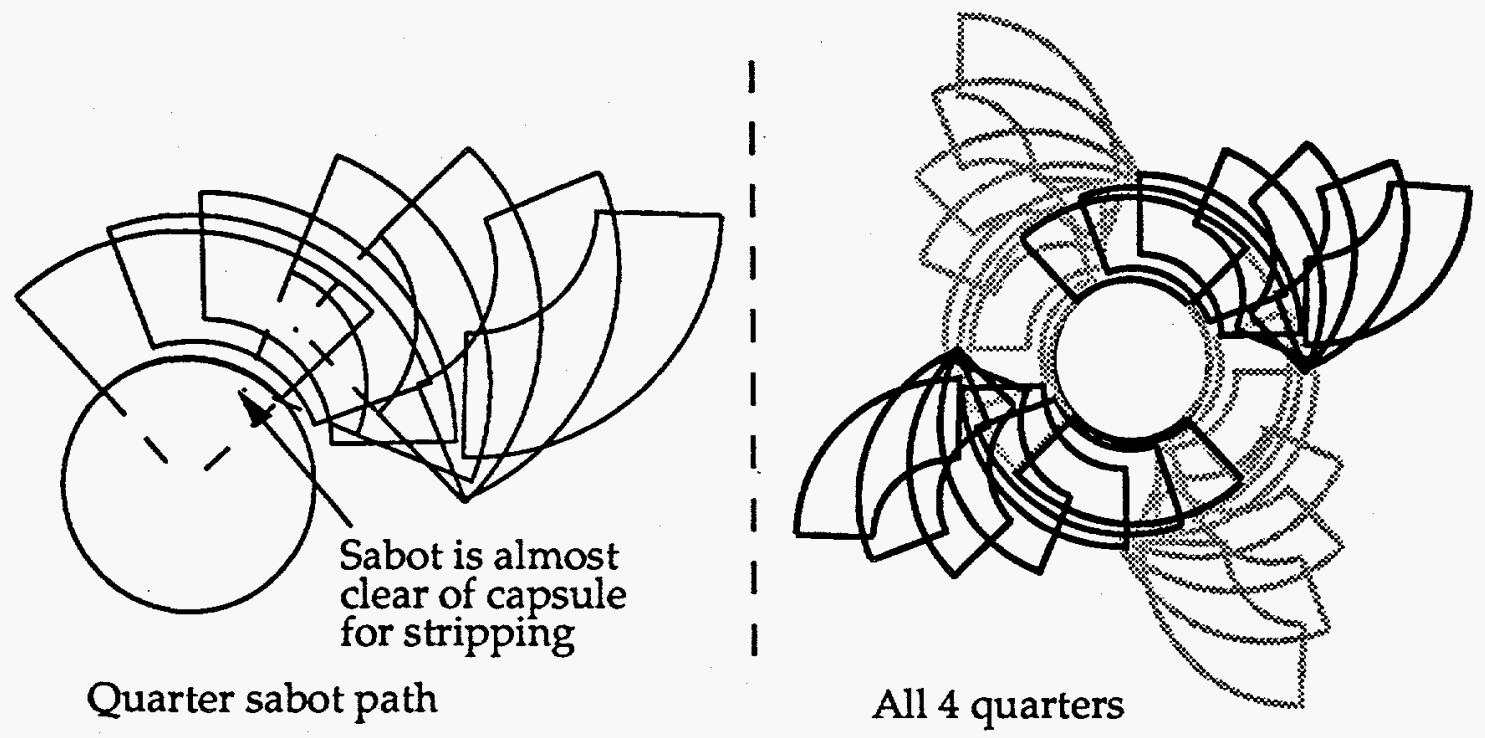

Figure 2-24b. Paths of a four piece sabot after leaving the gun barrel. The pieces are removed by centrifugal force. The dashed lines indicate the extent of the sabot at the ends where material is not removed for the capsule.

There are other methods of clearing sabots from capsules which may be used if centrifugal force proves undesirable. Springs could be inserted between the sabot pieces to force them apart. Gas pressure from the gun could be utilized for this purpose as well. 


\subsection{Summary of Injection Requirements}

The interdependent relationships between target acceleration $\left(1,800 \mathrm{~m} / \mathrm{s}^{2}\right.$ for a $0.1 \mu \mathrm{m}$ thick membrane), target speed $(100 \mathrm{~m} / \mathrm{s})$, reaction chamber size (3 $\mathrm{m}$ radius), target injector (acceleration) length $(3 \mathrm{~m})$, target coasting distance ( 7 $\mathrm{m})$, and interpulse time ( $0.17 \mathrm{~s})$ were described analytically and graphically (nominal values are given in parentheses).

The minimum target injection speed consistent with power plant pulse rate, chamber conditions, and target heating should normally be chosen for minimum injector length and ease of target tracking.

Accurate placement $(\sim 0.4 \mathrm{~mm})$ of driver beams on target is required to achieve high gain. This requires precise target tracking and beam pointing accuracy and limits allowed target tumble. Target tumble may be minimized by spinning the target (rifling the gun barrel) if tumble is experimentally determined to be excessive.

About $0.23 \mathrm{~m}$ of shielding could reduce neutron heating of targets in flight to about $0.1 \mathrm{~K}$ if one target is $5 \mathrm{~m}$ away when another is exploding. This shield could be a movable shield, a liquid injected between the targets and inside the chamber, or fixed shielding if the targets are sufficiently slow moving to have an adequately curved trajectory.

Prior to injection, the targets are cooled in cryogenic helium. During injection the target fuel temperature must be kept well below the triple point of $19.7 \mathrm{~K}$ (perhaps about $17.4 \mathrm{~K}$ for adequate mechanical strength). Target capsules, target injectors, sabots, and hohlraums must be designed to minimize DT fuel heating. Transient, finite element, 1-D spherical conduction calculations show that indirect drive target heating should not be excessive. Direct-drive targets heat more rapidly and may require higher 
injection speeds or reduced heat flux may be achieved by use of reflective material on the outer capsule surface. Convection heating was estimated for indirect-drive targets. However, more detailed studies including a proper time dependent 3-D computations including convection are recommended.

A sabot that uses centrifugal force for separation from a capsule should have more than two pieces to separate without imparting an uncontrolled momentum to the capsule.

Table 2-1 summarizes typical target injection requirements. Attainment of these requirements may be achievable but substantial further study and development is necessary.

\subsection{References}

1. P. C. Souers, Hydrogen Properties for Fusion Energy (University of California Press, Berkeley, CA 1986), pp. 57, 78-80, 96.

2. M. J. Monsler, "Laser Fusion: An Assessment of Pellet Injection, Tracking, and Beam Pointing," Proc. Third Topical Meeting on the Technology of Controlled Nuclear Fusion, Santa Fe, NM, May 9-11, 1978 (NTIS, Springfield, VA, 1978), pp. 1186-1197.

3. W. R. Meier et al., OSIRIS and SOMBRERO Inertial Confinement Fusion Power Plant Designs Final Report, W. J. Schafer Associates, Livermore, CA, DOE/ER/54100-1 (1992)Vol. II Ch. 4, Appendix A, see also R. F. Borque, W. R. Meier, and M. J. Monsler, "Overview of the OSIRIS IFE Reactor Conceptual Design", Fusion Technology, 21, 1465-1469 (1992).

4. R. W. Moir et al., "HYLIFE-II A Molten Sảlt Inertial Fusion Energy Power Plant Design-Final Report", Fusion Technology, 25(1), 5-25 (1994).

5. L. M. Waganer et al., Inertial Fusion Energy Reactor Design Studies, McDonnell Douglas Aerospace, Saint Louis, MO, DOE/ER-54101 (1992), Ch. 6.4, App. A, B, C. 
6. J. H. Pitts, R. F. Bourque, W. J. Hogan, W. R. Meier, and M. T. Tobin, The Cascade Inertial Confinement Fusion Reactor Concept, Lawrence Livermore National Laboratory, Livermore, CA, UCRL-LR-104546 (1990).

7. R. Beeman, Air Gun Digest-The complete book of pneumatic, spring, and $\mathrm{CO}_{2}$ weapons (DBI Books Inc., Northfield, $\mathrm{IL}, 1977$ ), p. 238.

8. L. D. Landau and E. M. Lifshitz, Mechanics, 3rd ed. (Pergamon Press, Oxford, 1976), p. 107.

9. T. J. McCarville, W. R. Meyer, C. F. Carson, and B. B. Glasgow, SAFIRE-A Systems Analysis Code for ICF Reactor Economics, Lawrence Livermore National Laboratory, Livermore, CA, UCRL-15872 (1987), vol. 1, p. 48.

10. D. S. Zuckerman et al., "An Induction Linac Driven Heavy-Ion Fusion Systems Model," Fusion Technology, 13(2), 217-254 (1988).

11. Inertial Confinement Fusion Central Station Electric Power Generating Plant, Final Report, U. S. Department of Energy, DOE/DP/40086-1 (1981); the Westinghouse ICECAP code is found in this report.

12. W. R. Meier et al., OSIRIS and SOMBRERO Inertial Fusion Power Plant Designs, W. J. Schafer Associates, Livermore, CA, DOE/ER/54100-1 (1992), Ch. 8.4.

13. B. G. Logan, R. W. Moir, and M. A. Hoffman, Requirements for Low Cost Electricity and Hydrogen Fuel Production from Multi-Unit Inertial Fusion Energy Plants with a Shared Driver and Target Factory, Lawrence Livermore National Laboratory, Livermore, CA, UCRL-JC-115787 (1994).

14. P. F. Peterson and V. E. Schrock, Experimental Investigation and Demonstration of IFE Target Injection and Tracking (Research Proposal written by R. W. Petzoldt), University of California, Berkeley, CA (1993).

15. E. F. Plechaty, D. E. Cullen, R. J. Howerton, and J. R. Kimlinger, Tabular and Graphical Presentation of 175 Neutron Group Constants Derived from 
the LLL Evaluated Neutron Data Library (ENDL), Lawrence Livermore National Laboratory, Livermore, CA, UCRL-50400 (1976), Vol.16.

16. J. Latkowski, Lawrence Livermore National Laboratory, Livermore, CA, Private Communication (1993).

17. D. D. Ho, J. A. Harte, and M. Tabak, "Radiation-Driven Targets for Heavy Ion Fusion," Presented at the Fifteenth International Conference on Plasma Physics and Controlled Nuclear Fusion Research, Seville, Spain, Sep. 26 - Oct. 2, 1994, IAEA-CN-60/B-P-13, to be published in Nuclear Fusion.

18. G. W. Collins, Lawrence Livermore National Laboratory, Livermore, CA, private communication (1994).

19. P. C. Souers, Hydrogen Properties for Fusion Energy (University of California Press, Berkeley, CA, 1986), pp. 85-86.

20. A. B. Shapiro, TOPAZ2D -- A Two-Dimensional Finite Element Code for Heat Transfer Analysis, Electrostatic, and Magnetostatic Problems, Lawrence Livermore National Laboratory, Livermore, CA, UCID-20824 (1986).

21. W. R. Meier et al., OSIRIS and SOMBRERO Inertial Fusion Power Plant Designs, W. J. Schafer Associates, Livermore, CA (1992), Vol. 2, chapter 4.3.

22. R. P. Reed and A. F. Clark, Materials at Low Temperatures (American Society for Metals, Metals Park, OH, 1983), pp. 48, 154.

23. P. C. Souers, Hydrogen Properties for Fusion Energy (University of California Press, Berkeley, CA, 1986), pp. 96, 102.

24. R. C. Weast ed., Handbook of Chemistry and Physics, 52nd ed. (The Chemical Rubber Co., Cleveland, OH, 1971), pp. E-3, E-207.

25. D. D. Ho, Lawrence Livermore National Laboratory, Livermore, Ca, private communication (1994). 
26. P. C. Souers, Hydrogen Properties for Fusion Energy (University of California Press, Berkeley, CA, 1986), p. 118-119.

27. M. Jakob, Heat-transfer (John Wiley \& Sons, New York, 1941) pp. 534-542.

28. R. C. Weast ed., Handbook of Chemistry and Physics, 52nd ed. (The Chemical Rubber Co., Cleveland, OH, 1971), p. F-44.

29. H. Lamb, Hydrodynamics, 6th ed. (Dover Publications, New York, 1932), p. 590. 


\section{Chapter 3 - Membrane Support of Accelerated Fuel Capsules}

\subsection{Introduction}

The primary purpose of this chapter is to provide a method to calculate the acceleration possible for an inertial fusion energy (IFE) fuel capsule that is supported by thin membranes. Inertial fusion energy target acceleration may be limited by mechanical failure of target materials. Two limiting materials are the capsule support membrane which must be very thin and the fuel (frozen DT) which has a low yield stress (fuel stress limitations are discussed in chapter 4). We also provide computational results and discuss the adequacy of these results to meet target injection requirements.

The fuel for IFE is deuterium and tritium (DT) frozen on the inner surface of small (a few millimeter radius) spherical capsules as in Fig. 1-1. The material for the capsule may be plastic (hydrocarbon), or glass, or other low atomic weight materials.

These capsules are injected into the center of a reaction chamber with wall temperature of roughly $1,000 \mathrm{~K}$. As noted in chapter 2 , the minimum required capsule injection speed is reactor repetition rate dependent but is nominally $100 \mathrm{~m} / \mathrm{s}$. For indirect-drive targets, it is necessary to suspend the capsule within a radiation case called a hohlraum. An example indirect-drive target ${ }^{1}$ which was designed by Darwin Ho is illustrated in Fig. 1-2. The shield, ring, and capsule all require support, but the capsule support will probably have the most effect on capsule implosion symmetry. One method to suspend the capsule in the hohlraum is to use a membrane as in Fig. 3-1a. Two (dual) membranes provide better overall support of the capsule as shown in Fig. 3-1b. Assuming that the driver energy can penetrate the 
membrane without too much non uniformity, the dual geometry is probably a very good membrane geometry. The dual membrane supports the capsule well in all directions. Frictional force would hold the capsule in place within the membrane. The bending stress in the membrane near the capsule is small. It has a relatively large membrane angle $(\theta)$ which allows the membrane to exert force more nearly in the direction of the target acceleration.

The entire assembly is called the target. Some parameters of a dual membrane supported target are defined in Fig. 3-2.

(a) Single membrane support

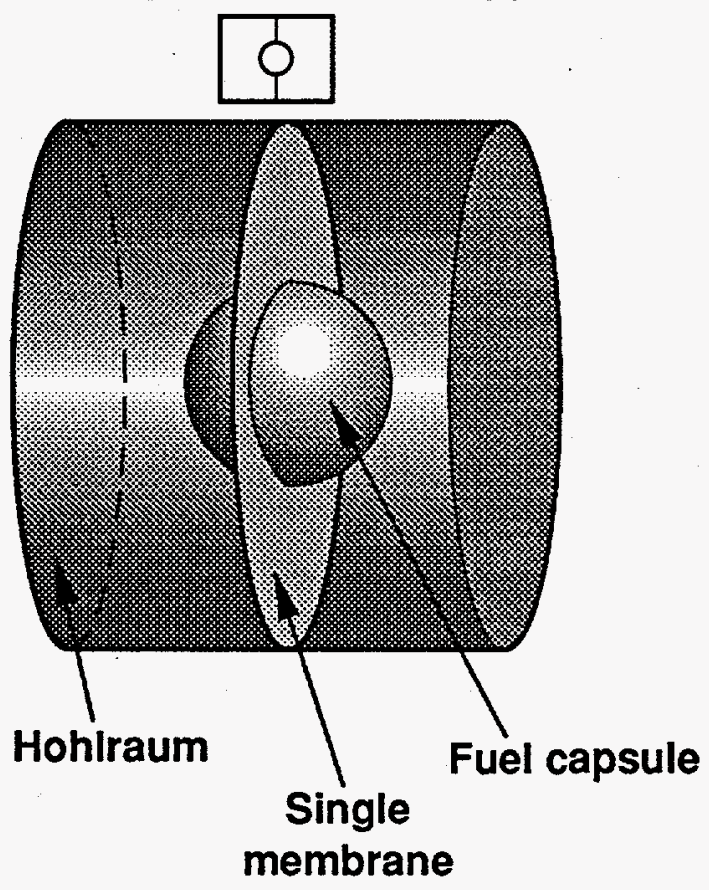

(b) Dual membrane support

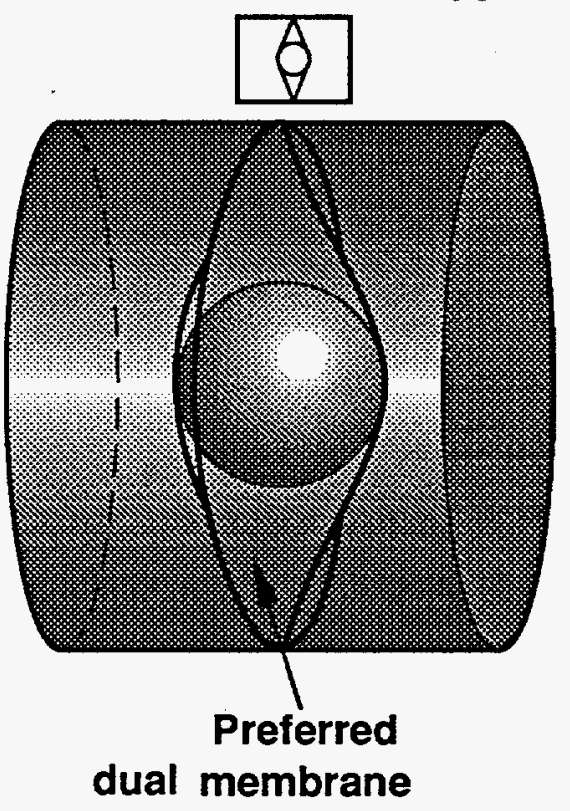

Figure 3-1. Targets with single (a) or dual (b) membrane support. 


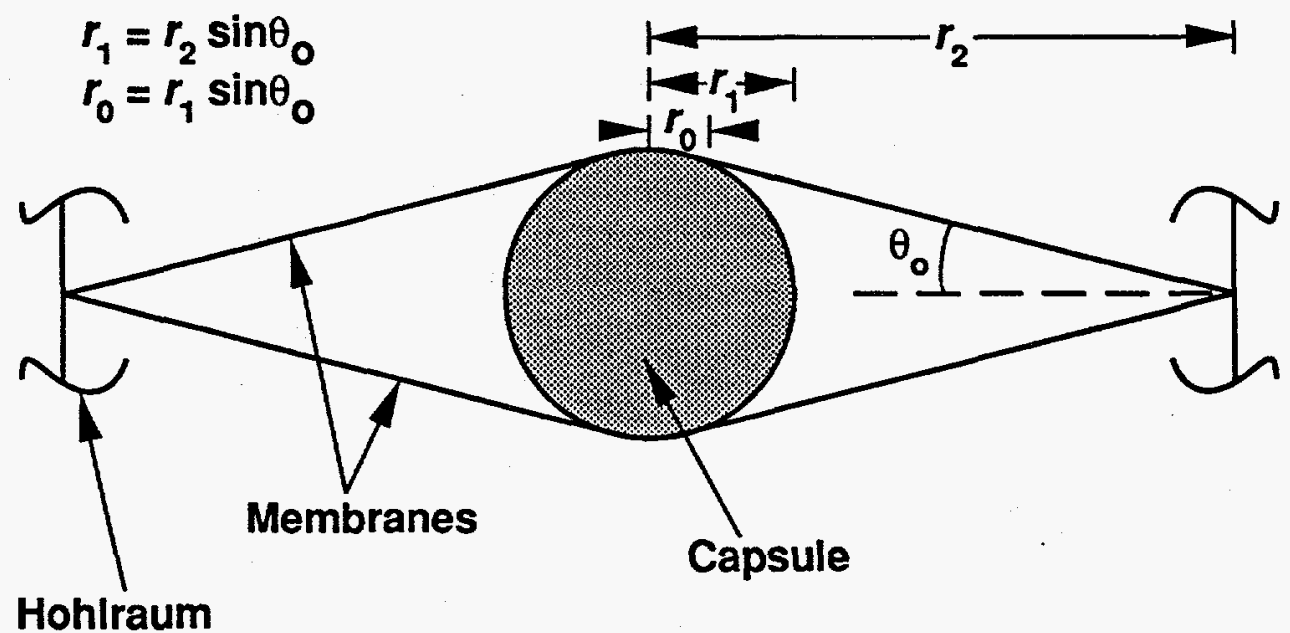

Figure 3-2. Dual membrane axisymmetric target configuration (rotated 90 degrees from Fig. 3-1).

\subsection{Effects of Membrane on Illumination Symmetry}

With indirect-drive targets, the driver energy directly or indirectly heats the inner walls of the hohlraum. The inner wall then radiates this energy to cause ablation of the external surface material of the capsule and a rapid implosion of the DT occurs. The resulting high temperature, high density DT then undergoes fusion reactions. To achieve optimum fuel density and temperature, the illumination of the capsule must be nearly uniform. For many target designs, the peak to minimum intensity variation must be held to no more than about 1 to $2 \%$.

The membranes are in the radiation path between the hohlraum walls and the capsule. Radiant energy, in the form of soft $x$ rays, that approaches the capsule from a direction parallel to the membrane will be strongly attenuated. Implosion instabilities (Rayleigh - Taylor) could arise due to uneven shadowing of the capsule due to the membranes, reducing the uniformity of the fuel implosion. These instabilities are difficult to calculate and are not quantitatively well understood. However, the instabilities are minimized if 
the membrane thickness is much less than the capsule thickness. NOVA targets use $0.1 \mu \mathrm{m}$ thick membranes. Further study will be required to quantify the allowed membrane thickness. Thicker membranes may be acceptable for larger reactor size targets but conservatively, $0.1 \mu \mathrm{m}$ is our base case membrane thickness.

Throughout this chapter, it is assumed that the hohlraum is a rigid body that supports the membranes. This assumption is justified since the hohlraum can be made sufficiently strong.

\subsection{Membrane Problem Description}

We assume that the target is accelerated along its axis of symmetry and neglect the mass of the membrane. The effects of membrane vibrations within the target are considered later in section 3.5. The membrane exerts a force on the capsule which accelerates the capsule along with the target. By reaction the capsule exerts a force on the membrane. This causes the membrane to deform in an axisymmetric manner as shown in Fig. 3-3. The membrane is assumed to be straight in the meridianal direction prior to capsule displacement. The initial angle of the membrane relative to the target axis $\theta_{0}$ is given by

$$
\theta_{o}=\arcsin \left(\frac{r_{1}}{r_{2}}\right)
$$

The height of the attachment point of the membrane above the center of the capsule $\eta_{\mathrm{o}}$ is given by

$$
\eta_{o}=r_{1} \cos \theta_{o}=r_{1} \sqrt{1-\left(\frac{r_{1}}{r_{2}}\right)^{2}}
$$



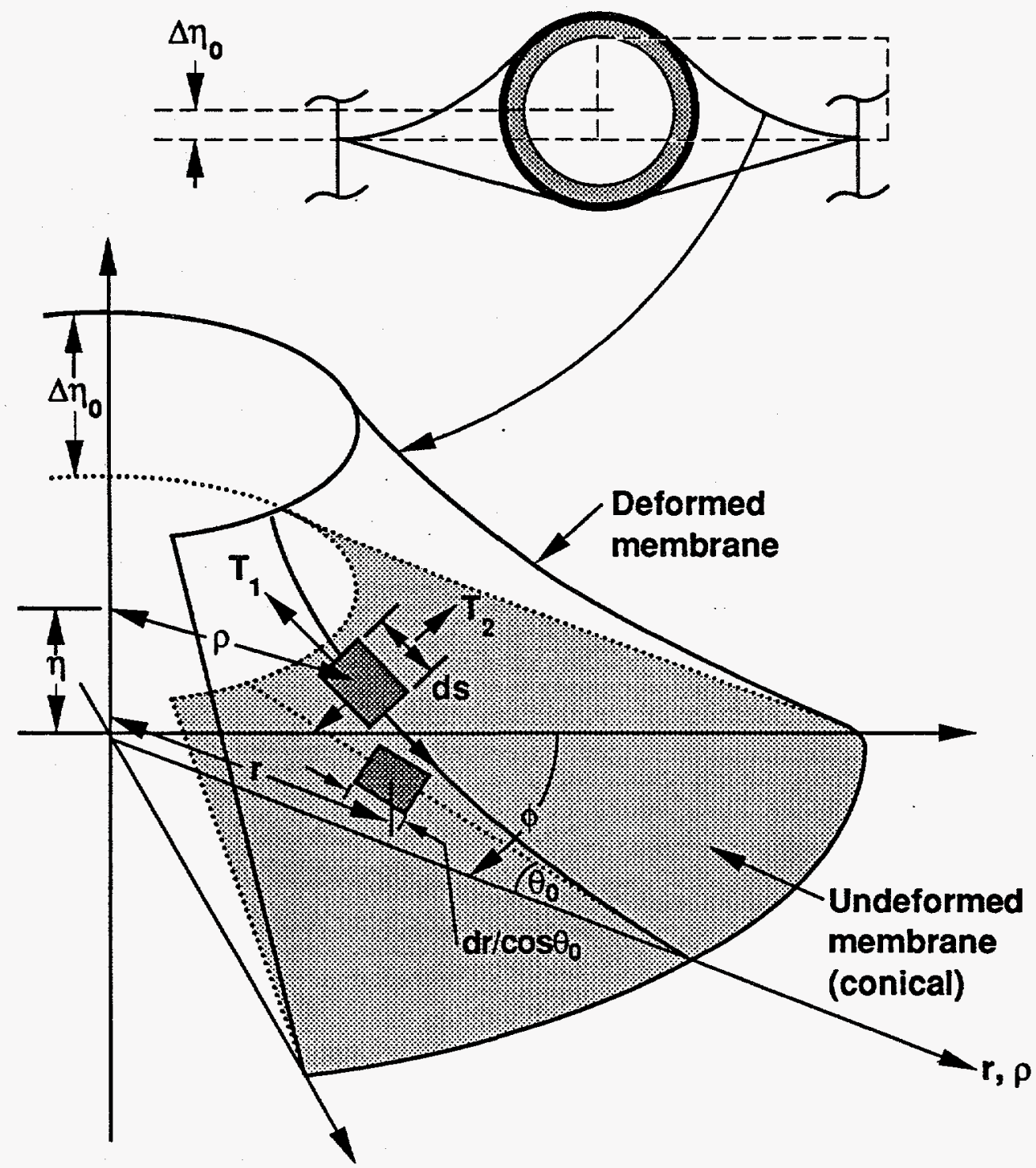

05-00-1194-3677pb01

Figure 3-3. Target with membrane deformation due to acceleration.

The membrane is thin (perhaps about $0.1 \mu \mathrm{m}$ ) to reduce the effect that the membrane has on target implosion symmetry. The capsule and hohlraum are assumed to be much stronger and more rigid than the membrane. Therefore, one limit on target acceleration is membrane strength including how much stress and strain the membrane can withstand without damage. 
Yield stress and strain vary with material and with temperature. Figure 34 shows stress vs strain characteristics of mylar $\left(\mathrm{C}_{10} \mathrm{H}_{8} \mathrm{O}_{4}\right)_{\mathrm{n}}$ for a variety of temperatures ${ }^{2}$. The operating temperature of interest is about $17 \mathrm{~K}$ because of the use of cryogenic fuel. For mylar between $4 \mathrm{~K}$ and $20 \mathrm{~K}$ the stress-strain curve is nearly linear up to the ultimate stress of the material \{about $300 \mathrm{MPa}$ at strain of 0.04). There is little indication of yielding behavior, particularly at strain less than 0.02 . A maximum strain value of 0.02 would give a safety factor of 2 below the ultimate strain of 0.04 . It would not be catastrophic if an occasional membrane broke, causing a 'dud' target. However, plant energy efficiency would be degraded, so excessive failures should be avoided. 


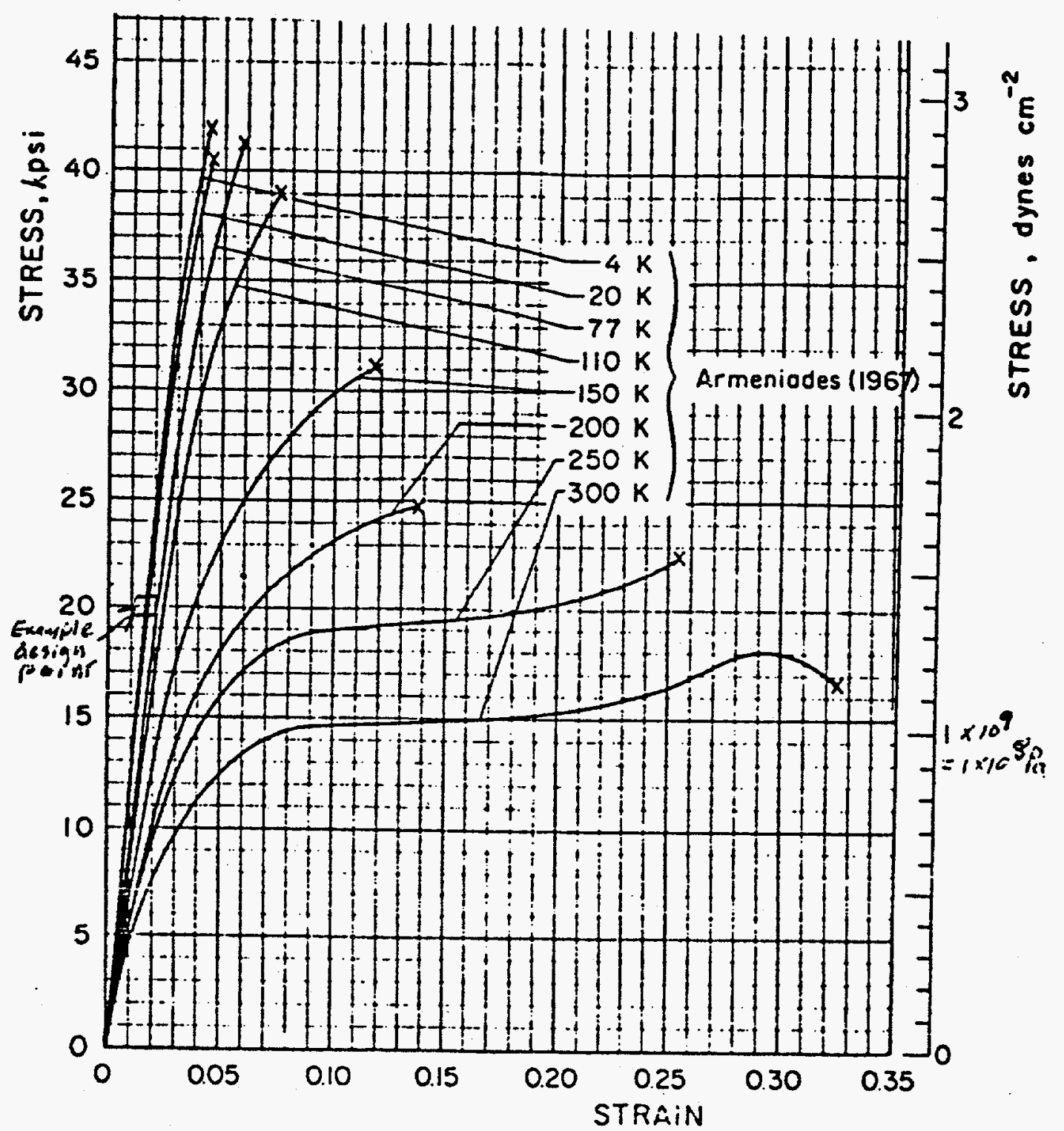

Figure 3-4. Stress vs strain for mylar at various temperatures (Source: Schramm, Clark, and Reed. A compilation and evaluation of mechanical, electrical, and thermal properties of selected polymers. Original source: Armeniades, Kuriyama, Roe, and Baer 1967).

There is also an uncertainty in capsule location related to the amplitude of the capsule oscillations within the hohlraum. As will be shown later, these 
oscillations can be reduced by gradually (rather than abruptly) reducing the acceleration near the end of the target injector.

The goal is to determine the maximum acceptable target acceleration and minimum acceptable membrane thickness. To do this we must calculate the membrane strain and displacement.

\subsection{Formulation and Results}

In this section we apply both linear and non-linear membrane theory to calculate the force and displacement exerted by a membrane on a capsule. The membrane strain, displacement, and rotation are small enough that linear theory gives reasonable results. However, the linear theory can not satisfy the assumed boundary conditions without including bending effects near the boundaries.

\subsubsection{Linear Formulation}

The linear formulation follows the work of Timoshenko and Woinowsky- Krieger ${ }^{3}$ on membrane theory of shells of revolution. A shell of revolution (e.g. the membrane supporting an IFE capsule) is defined by rotation of a plane curve about an axis in the plane of the curve. The curve is called the meridian and its plane the meridian plane. Two dimensional slices of the shell perpendicular to the axis form parallel circles. An element of the shell is bounded by two adjacent meridians and two adjacent parallel circles as shown in Fig. 3-5. The geometry and applicable forces are indicated. The radius of the parallel circle is $r_{3}$, the radius of curvature of the membrane in the meridianal direction is $r_{4}$, and the distance (normal to the membrane) from the element to the axis of symmetry is $r_{5}$. The elements position and orientation are further defined by the angle $\Phi$ relative to a reference meridian and angle $\theta$ that the normal to the element makes with the axis. The normal 
forces per unit edge length acting on the membrane are $T_{1}$ and $T_{2}$ as shown. One corner of the element can define a coordinate axis with $\mathrm{x}$ axis tangent to the parallel circle, $y$ axis tangent to the meridian, and $z$ axis the inward normal to the membrane at that point. With external loads per unit area $Y$ in the $y$ direction and $Z$ in the $z$ direction, an equilibrium equation in the $y$ direction yields 3

$$
\frac{d}{d \theta}\left(T_{1} r_{3}\right)-T_{2} r_{4} \cos \dot{\theta}+Y r_{4} r_{3}=0
$$

and an equilibrium equation in the $\mathrm{z}$ direction yields

$$
T_{1} r_{3}+T_{2} r_{4} \sin \theta+Z r_{4} r_{3}=0
$$

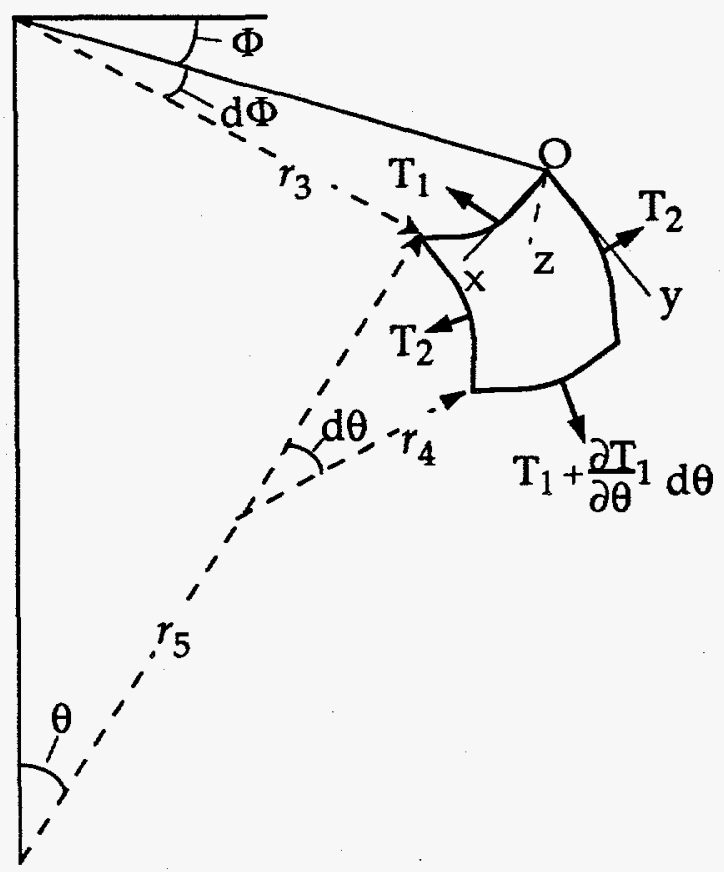

Figure 3-5. Geometry and forces applicable to an axisymmetric membrane.

In the case of a cone with no external load (which is the assumed initial condition of the membrane) $r_{4}$ becomes infinite. As shown in Fig. 3-6, $y$ can 
be redefined as the distance from the axis in a direction parallel to the membrane. $y$ and $r_{3}$ are simply related by the equation

$$
r_{3}=y \cos \theta
$$

Equations (3-3) and (3-4) then simplify to

$$
T_{1} r_{3}=C_{1}
$$

where $C_{1}$ is a constant and $T_{2}=0$. The constant $C_{1}$ is related to the net axial force $F$ exerted by the membrane on the capsule.

$$
F=2 \pi r_{3} T_{1} \sin \theta=2 \pi \mathrm{C}_{1} \sin \theta \text { so } C_{1}=F / 2 \pi \sin \theta
$$

To calculate the assumed small displacement of the membrane elements, the displacement is broken into components. One component $v_{1}$ is tangential to the membrane in the $y$ direction, the other component $w_{1}$ is perpendicular to the membrane in the $z$ direction. The change in $v_{1}$ over an element $\mathrm{d} v_{1}$ is given by

$$
d v_{l}=\frac{d v_{l}}{d y} \frac{d y}{d r_{3}} d r_{3}=\varepsilon_{1} \frac{1}{\cos \theta} d r_{3}
$$

where $\varepsilon_{1}$ is the meridianal strain and is given by Hooke's law

$$
\varepsilon_{1}=\frac{1}{E h}\left(T_{1}-v T_{2}\right)=\frac{T_{1}}{E h}
$$


where $E$ is Young's modulus, $h$ is the membrane thickness, $v$ is Poisson's ratio, and $T_{2}=0$. Since the outer edge of the membrane is attached to the hohlraum, $v_{1}$ and $w_{1}$ are assumed to be zero at the outer boundary (note $\left.v_{l}, w_{l} \leq 0\right)$. Substituting equations (3-6), (3-7), and (3-9) into equation (3-8) and integrating gives

$$
\begin{aligned}
v_{l}\left(r_{3}\right)= & \int_{r_{3 \max }}^{r_{3}} \frac{F}{2 \pi \sin \theta \cos \theta E h r_{3}} d r_{3}^{\prime}=\frac{F}{2 \pi \sin \theta \cos \theta E h} \ln \left(\frac{r_{3}}{r_{3 \max }}\right) . \\
& =\frac{2 \pi r_{3} T_{1} \sin \theta}{2 \pi \sin \theta \cos \theta E h} \ln \left(\frac{r_{3}}{r_{3 \max }}\right)=\frac{r_{3} \varepsilon_{1}}{\cos \theta} \ln \left(\frac{r_{3}}{r_{3 \max }}\right)
\end{aligned}
$$

An inconsistency arises when we try to compute $w_{1}$. Due to the displacements $v_{1}$ and $w_{1}$, the radius of the parallel circle $r_{3}$ changes by an amount $v_{l} \cos \theta-w_{l} \sin \theta$. The circumference increases in direct proportion to the radius so

$$
\varepsilon_{2}=\frac{1}{r_{3}}\left(v_{l} \cos \theta-w_{l} \sin \theta\right)
$$

where $\varepsilon_{2}$ is the circumferential strain. By Hooke's law

$$
\varepsilon_{2}=\frac{1}{E h}\left(T_{2}-v T_{1}\right)=\frac{-v T_{1}}{E h}
$$

does not accommodate the condition of zero circumferential strain at the outer boundary since $T_{2}$ vanishes for a cone and $T_{1}$ does not vanish. For the special case of zero Poisson's ratio, circumferential strain vanishes and equation (3-11) reduces to 


$$
w_{l} \approx v_{l} \cot \theta
$$

In this case, the net displacement $\Delta \eta$ of all points on the membrane is in the axial direction only and is given by

$$
\Delta \eta=\sqrt{v_{l}^{2}+w_{l}^{2}}=-v_{l} \sqrt{1+\cot ^{2} \theta}=\frac{-v_{l}}{\sin \theta}=\frac{-r_{3} \varepsilon_{1}}{\sin \theta \cos \theta} \ln \left(\frac{r_{3}}{r_{3 \max }}\right)
$$

The final shape is no longer a true cone but is curved in the meridianal direction. Applying equation (3-14) to a membrane supported capsule of radius $r_{1}$ in a hohlraum of radius $r_{2}$ with radius of membrane contact $r_{0}$, we find that

$$
\theta=\arcsin \left(\frac{r_{1}}{r_{2}}\right), r_{2}=r_{3 \max }, \text { and } r_{0}=r_{3 \min }=\frac{r_{1}^{2}}{r_{2}}
$$

Substituting equations (3-15) into equations (3-14) and dividing through by $r_{2} \varepsilon_{1}$ to normalize the results, we can obtain an equation for the membrane displacement at radius $r_{0}$, which is equivalent to the displacement of the capsule within the hohlraum.

$$
\frac{\Delta \eta_{0}}{\varepsilon_{1} r_{2}}=\frac{-r_{1}}{r_{2} \cos \left(\arcsin \left(\frac{r_{1}}{r_{2}}\right)\right)} \ln \left(\frac{r_{1}}{r_{2}}\right)^{2}
$$




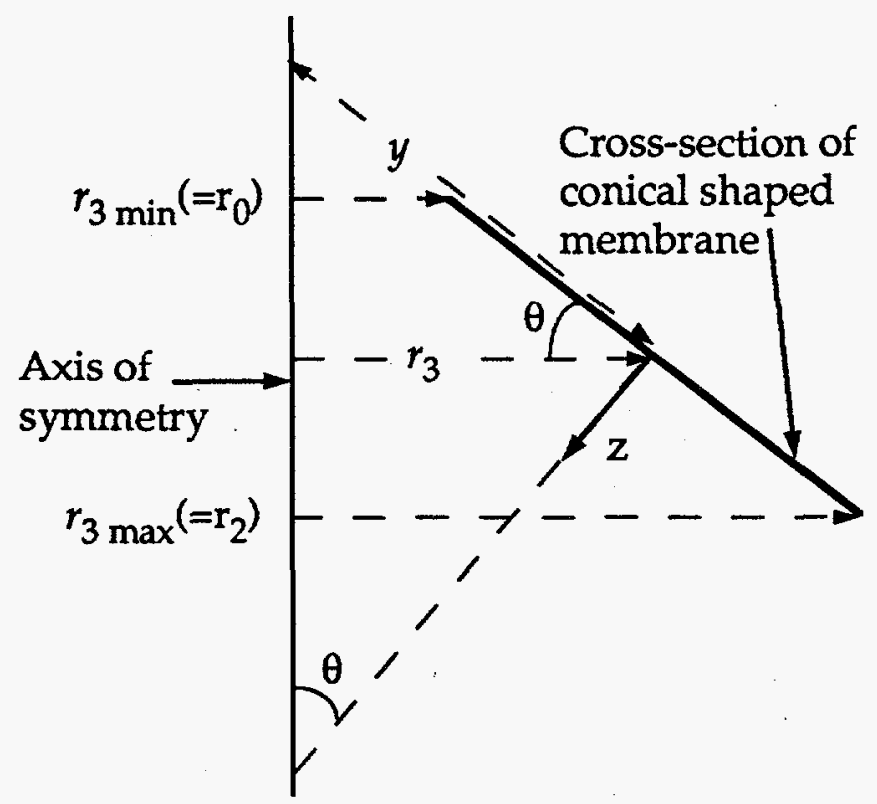

Figure 3-6. Geometry of conical membrane.

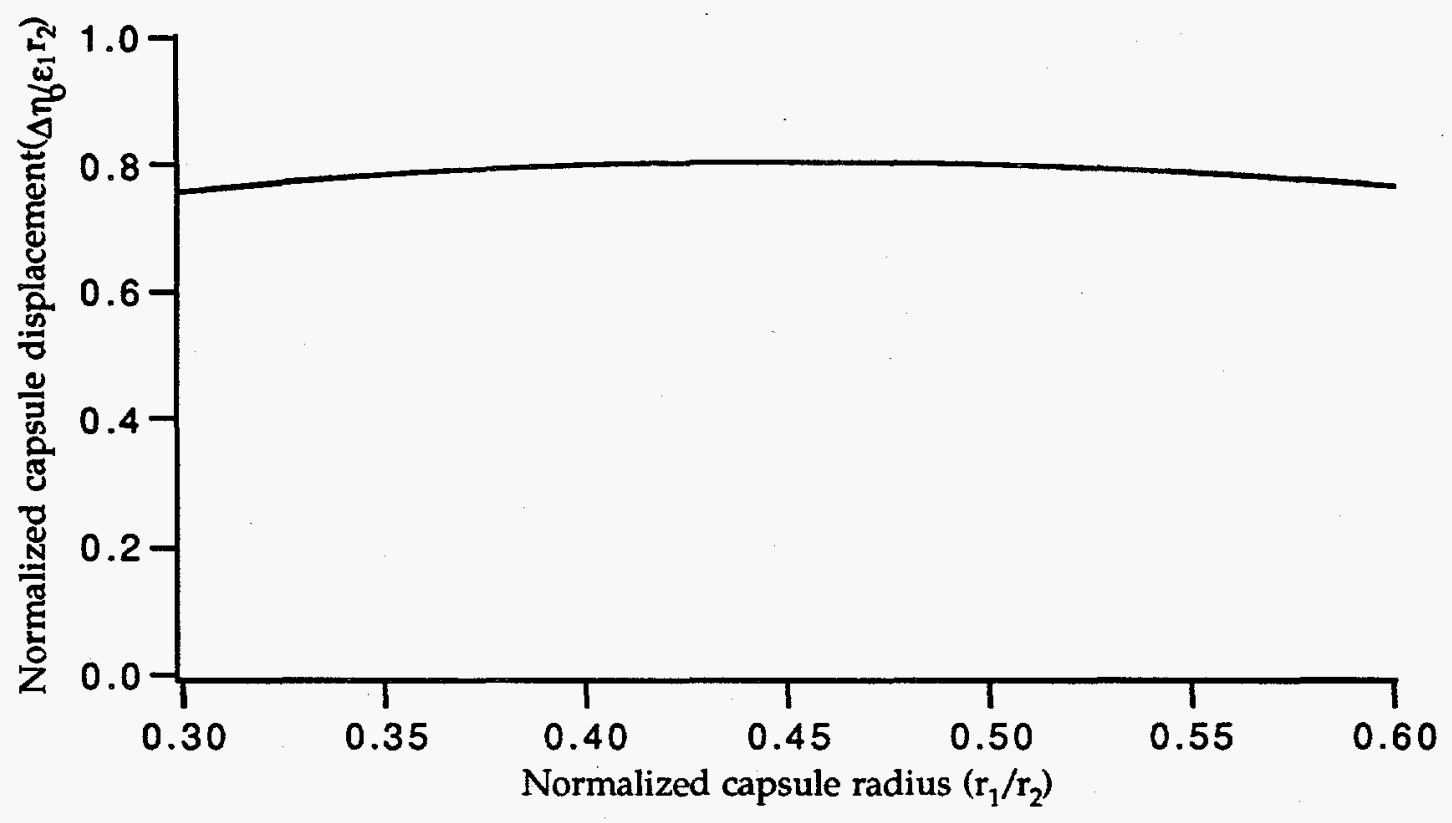

Figure 3-7. Normalized capsule displacement vs normalized capsule radius.

The normalized capsule displacement from equation (3-16) is plotted in Fig. 3-7 for a range of normalized capsule sizes. Capsule displacement is nearly independent of capsule radius over the range of interest from 0.3 to 0.6 
times the hohlraum radius. The expected normalized capsule radius is about 0.4 .

\subsubsection{Nonlinear Formulation}

Yang and Feng 4 investigated axisymmetric deformations of nonlinear (nonlinear stress-strain relationship) membranes and developed differential equations that are appropriate to this problem. Their method requires several definitions which will be described here. Figure 3-3 shows the deformation geometry.

As shown in Fig. 3-3, the undeformed membrane is assumed to be conical in shape to the circle at which it contacts the capsule. $r$ and $\Phi$ are the polar coordinates of a point on the undeformed membrane. The height of a point on the undeformed membrane is given by $\eta_{u}=\left(r_{2}-r\right) \tan \theta_{0}$. In this axisymmetric problem the properties of interest do not depend on polar angle $\Phi$. An infinitesimal element of the undeformed membrane has meridianal dimension $d r / \cos \theta_{0}$ and circumferential dimension $r(d \Phi)$. The undeformed membrane thickness is $h$. Upon deformation the element $d r$ at radius $r$ moves to deformed radius $\rho$ and height $\eta$. The deformed element length is ds. The meridianal and circumferential stress resultants (force per unit edge length of the membrane) are $T_{1}$ and $T_{2}$.

The stretch ratios in the meridianal, circumferential, and perpendicular to membrane directions are denoted by $u, v$, and $x$ respectively. Then

$$
u=\frac{d s\left(\cos \theta_{0}\right)}{\mathrm{d} r} \text { and } v=\frac{\rho}{r} \text {. }
$$

We define additional variables 


$$
w=\frac{d \rho}{d r} \text { and } y=\frac{d s}{d r}=\frac{u}{\cos \theta_{o}}
$$

In equilibrium the net force on a differential element of the membrane is equal to zero. Consider a differential element of the membrane shown in Figure 3-8.

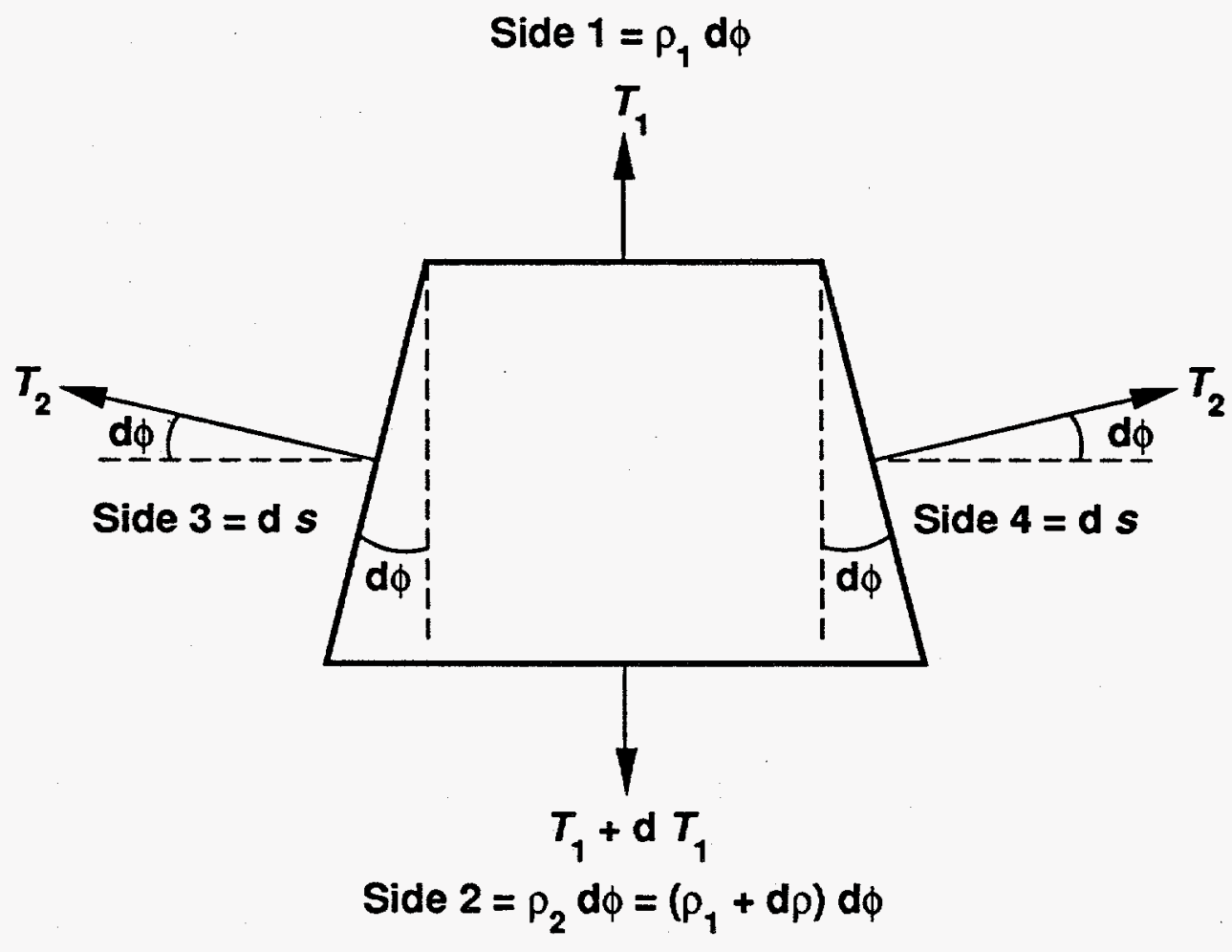

Figure 3-8. A differential element of the deformed membrane.

Due to the membrane curvatures, it is difficult to properly illustrate the angle $d \phi$ in this figure. $T_{2} \|$ (the projection of $T_{2}$ in the direction of $T_{1}$ ) is used to define $d \phi$.

$$
T_{2} \|=T_{2} \sin d \phi .
$$


$d \rho$ is given by

$$
d \rho=\rho_{2}-\rho_{1}
$$

And $d \phi$ is given by

$$
\sin d \phi=d \phi=\frac{d \Phi}{2} \frac{d \rho}{d s}
$$

The net force in the meridianal direction equals zero. This implies

$$
\left(T_{1}+d T_{1}\right)\left(\rho_{1}+d \rho\right) d \Phi=T_{1} \rho_{1} d \Phi_{1}+2 T_{2} d s \sin d \phi \text { or },
$$

$T_{1} \rho_{1} d \Phi+T_{1} d \rho d \Phi+d T_{1} \rho_{1} d \Phi+d T_{1} d \rho d \Phi=T_{1} \rho_{1} d \Phi_{1}+2 T_{2} d s \frac{d \Phi}{2} \frac{d \rho}{d s}$

Neglecting $d T_{1} d \rho d \Phi$ which is much less than the remaining terms and dividing by $\rho d \Phi d \rho$ gives

$$
\frac{d T_{1}}{d \rho}+\frac{1}{\rho}\left(T_{1}-T_{2}\right)=0
$$

Similarly, the net force perpendicular to the membrane is zero. This leads to

$$
\mathrm{K}_{1} T_{1}+\mathrm{K}_{2} T_{2}=0
$$

where $K_{1}$ and $K_{2}$ are the principle curvatures in the meridianal and circumferential directions, respectively.

We can now solve for $K_{1}$ and $K_{2}$ in terms of $y, v$, and $w$. 
Let $\theta$ be the angle between the membrane and the plane perpendicular to the axis of symmetry. Then

$$
\cos \theta=\frac{d \rho}{d s} \text { or } \theta=\cos ^{-1}\left(\frac{d \rho}{d s}\right) \text {. }
$$

To evaluate $K_{1}$, use

$$
\mathrm{K}_{1} \equiv \frac{d}{d s}(\theta)=\frac{d}{d s}\left(\cos ^{-1}\left(\frac{d \rho}{d s}\right) .\right.
$$

But for any variable $t$,

$$
\frac{d}{d s} \cos ^{-1}(t)=\frac{-1}{\sqrt{1-t^{2}}} \frac{d t}{d s} .
$$

So,

$$
\mathrm{K}_{1}=\frac{-1}{\sqrt{1-\left(\frac{d \rho}{d s}\right)^{2}}} \frac{d}{d s}\left(\frac{d \rho}{d s}\right)=\frac{-1}{\sqrt{1-\left(\frac{d \rho}{d r} \frac{d r}{d s}\right)^{2}}} \frac{d r}{d s} \frac{d}{d r}\left(\frac{d \rho}{d r} \frac{d r}{d s}\right) .
$$

But

$$
\frac{d \rho}{d r}=w \text { and } \frac{d r}{d s}=\frac{1}{y}
$$

so

$$
K_{1}=\frac{-1}{\sqrt{1-\frac{w^{2}}{y^{2}}}} \frac{1}{y}\left(w y^{-1}\right)^{\prime}=\frac{-1}{\sqrt{y^{2}-w^{2}}}\left(w^{\prime} y^{-1}-w y^{-2} y^{\prime}\right)=\frac{w y^{\prime}-y w^{\prime}}{y^{2} \sqrt{y^{2}-w^{2}}}
$$

Here the primes denote differentiation with respect to $r$. 
To evaluate $\mathrm{K}_{2}$, use

$$
\begin{gathered}
K_{2}=\frac{1}{\rho} \sin \theta=\frac{1}{\rho}\left( \pm \sqrt{1-\left(\frac{d \rho}{d s}\right)^{2}}\right)=\frac{1}{\rho}\left( \pm \sqrt{1-\left(\frac{d \rho}{d r} \frac{d r}{d s}\right)^{2}}\right), \text { or } \\
K_{2}=\frac{1}{r v}\left( \pm \sqrt{1-\left(\frac{w}{y}\right)^{2}}\right)= \pm \frac{\sqrt{y^{2}-w^{2}}}{r v y} .
\end{gathered}
$$

In the target membrane problem $\mathrm{K}_{2}$ is positive, so the positive sign applies above.

For two-dimensional stresses in an elastic material, $T_{1}$ and $T_{2}$ will be functions of the stretch ratios $u$ (or $y=u / \cos \theta_{0}$ ) and $v$, i.e.

$$
T_{1}=T_{1}(y, v) \text { and } T_{2}=T_{2}(y, v)
$$

We now solve for the derivatives of $y, v$, and $w$ with respect to $r$. Recall equation (3-24),

$$
\frac{d T_{1}}{d \rho}+\frac{1}{\rho}\left(T_{1}-T_{2}\right)=0
$$

But

$$
\frac{d T_{1}}{d \rho}=\frac{\partial T_{1}}{\partial y} \frac{d y}{d \rho}+\frac{\partial T_{1}}{\partial v} \frac{d v}{d \rho}
$$

with 


$$
\frac{d v}{d \rho}=\frac{d}{d \rho}\left(\frac{\rho}{r}\right)=\frac{1}{r}-\frac{\rho}{r^{2}} \frac{d r}{d \rho}=\frac{1}{r}-\frac{v}{w r}
$$

and

$$
\frac{d}{d \rho}=\frac{d r}{d \rho} \frac{d}{d r}=\frac{1}{w} \frac{d}{d r}
$$

so

$$
\frac{d y}{d \rho}=\frac{1}{w} \frac{d y}{d r}
$$

Define

$$
f_{1} \equiv \frac{\partial T_{1}}{\partial y}, f_{2} \equiv \frac{\partial T_{1}}{\partial v} \text {, and } f_{3} \equiv\left(T_{2}-T_{1}\right)
$$

Substituting equations (3-35, 3-36, 3-38, and 3-39) into (3-24), the equilibrium equation in the meridianal direction, gives

$$
\frac{\mathrm{f}_{1} d y}{w d r}+\mathrm{f}_{2}\left(\frac{1}{r}-\frac{v}{w r}\right)-\frac{\mathrm{f}_{3}}{r v}=0 .
$$

Solving for $\frac{d y}{d r}$ gives

$$
\frac{d y}{d r}=\frac{f_{3} w}{f_{1} r v}+\frac{f_{2}}{f_{1} r}(v-w)
$$

Now solve for $\frac{d v}{d r}$. 


$$
\frac{d v}{d r}=\frac{d}{d r}\left(\frac{\rho}{r}\right)=\frac{1}{r} \frac{d \rho}{d r}-\frac{\rho}{r^{2}}=\frac{1}{r}(w-v)
$$

Now solve for $\frac{d w}{d r}=w^{\prime}$. Recall equation (3-25), the equilibrium equation perpendicular to the membrane, $\mathrm{K}_{1} T_{1}+\mathrm{K}_{2} T_{2}=0$. Substituting for $\mathrm{K}_{1}$ and $\mathrm{K}_{2}$ gives

$$
\frac{w y^{\prime}-y w^{\prime}}{y^{2} \sqrt{y^{2}-w^{2}}} T_{1}+\frac{\sqrt{y^{2}-w^{2}}}{y v r} T_{2}=0
$$

or

$$
\left(w y^{\prime}-y w^{\prime}\right)=\frac{-\left(y^{2}-w^{2}\right) y T_{2}}{v r T_{1}}
$$

so

$$
\frac{d w}{d r}=w^{\prime}=\frac{w y^{\prime}}{y}+\frac{\left(y^{2}-w^{2}\right) T_{2}}{v r T_{1}}
$$

To apply these differential equations, the constitutive equations for $T_{1}=$ $T_{1}(y, v)$ and $T_{2}=T_{2}(y, v)$ are needed. As was done in reference 4 , we assume the Mooney model applies for the membrane material. Strain energy $W$ is the energy associated with the deformation of an elastic material, such as the energy of a stretched spring. A Mooney model material has a strain energy function of the form

$$
W\left(I_{1}, I_{2}\right)=C\left[\left(I_{1}-3\right)+\alpha\left(I_{2}-3\right)\right]
$$


where

$$
\begin{gathered}
\mathrm{I}_{1}=u^{2}+v^{2}+x^{2}, \\
\mathrm{I}_{2}=u^{2} v^{2}+v^{2} x^{2}+u^{2} x^{2},
\end{gathered}
$$

$C$ is a constant with dimensions of energy per volume $(C$ is proportional to the modulus of elasticity for small strain), and $\alpha$ is a dimensionless constant relating the relative effect of $I_{1}$ and $I_{2}$.

A Mooney model material is also incompressible so that $u v x=1$.

This leads to the following constitutive equations ${ }^{4}$ for $T_{1}$ and $T_{2}$.

$$
\begin{aligned}
& T_{1}=2 \operatorname{Ch}\left(\frac{u}{v}-\frac{1}{u^{3} v^{3}}\right)\left(1+\alpha v^{2}\right) \text { and } \\
& T_{2}=2 \operatorname{Ch}\left(\frac{v}{u}-\frac{1}{u^{3} v^{3}}\right)\left(1+\alpha u^{2}\right) .
\end{aligned}
$$

Recall, $\mathrm{h}$ is the undeformed membrane thickness. To first order in the strain, these relationships for $T_{1}$ and $T_{2}$ reduce to Hooke's law. This equivalence will now be demonstrated for small strain and incompressible material (Poisson's ratio $v=1 / 2$ for an incompressible material).

$$
\begin{aligned}
\mathrm{T}_{1} & =2 \operatorname{Ch}\left(\frac{u}{v}-\frac{1}{u^{3} v^{3}}\right)\left(1+\alpha v^{2}\right) \\
& =2 \operatorname{Ch}\left[\frac{1+\varepsilon_{1}}{1+\varepsilon_{2}}-\frac{1}{\left(1+\varepsilon_{1}\right)^{3}\left(1+\varepsilon_{2}\right)^{3}}\right]\left[1+\alpha\left(1+\varepsilon_{2}\right)^{2}\right] \\
& \approx 2 \operatorname{Ch}\left[\left(1+\varepsilon_{1}\right)\left(1+\varepsilon_{2}\right)^{-1}-\left(1+3 \varepsilon_{1}\right)^{-1}\left(1+3 \varepsilon_{2}\right)^{-1}\right]\left[1+\alpha+2 \alpha \varepsilon_{2}\right] \\
& \approx 2 \operatorname{Ch}\left[\left(1+\varepsilon_{1}\right)\left(1-\varepsilon_{2}\right)-\left(1-3 \varepsilon_{1}\right)\left(1-3 \varepsilon_{2}\right)\right][1+\alpha] \\
& \approx 2 \operatorname{Ch}\left[\left(1+\varepsilon_{1}\right)-\varepsilon_{2}-1+3 \varepsilon_{1}+3 \varepsilon_{2}\right](1+\alpha) \\
& =2(1+\alpha) \operatorname{Ch}\left[4 \varepsilon_{1}+2 \varepsilon_{2}\right]
\end{aligned}
$$




$$
\begin{aligned}
& =8(1+\alpha) C h\left[\varepsilon_{1}+\frac{1}{\left.2^{\varepsilon_{2}}\right]}\right. \\
& =T_{1} \text { from Hooke's law. }
\end{aligned}
$$

The last step identified the constant $8(1+\alpha) C$ as being equal to $\frac{E}{1-v^{2}}$. This equality is valid for $\varepsilon_{1}$ and $\varepsilon_{2} \ll 1$ so that quantities of higher than first order in strain are negligible.

Typical experimental values 5 for $\alpha$ are less than $\frac{1}{8}$. The calculations are not sensitive to the choice of $\alpha$ for small strains. The results reported in section 3.4.2 use Mooney's model with $\alpha=0$. ( $C=E / 6$ for $v=1 / 2$ and $\alpha=0$.) Using Mooney's model and setting $\alpha=0$ in equations (3-49 and 3-50) and using $u=y \cos \theta_{0}$ gives

$$
\begin{gathered}
f_{1}=\frac{\partial T_{1}}{\partial y}=2 \operatorname{Ch} \cos \theta_{0}\left(\frac{1}{v}+\frac{3}{u^{4} v^{3}}\right) \\
f_{2}=\frac{\partial T_{1}}{\partial v}=2 \operatorname{Ch}\left(\frac{-u}{v^{2}}+\frac{3}{u^{3} v^{4}}\right) \\
f_{3}=2 C h\left(\frac{v}{u}-\frac{u}{v}\right)
\end{gathered}
$$

Substituting equations (3-49 through 3-53) into equations (3-41, 3-42, and 3-45) gives

$$
\frac{d y}{d r}=\frac{\left(\frac{v}{u}-\frac{u}{v}\right) w+\left(\frac{-u}{v}+\frac{3}{u^{3} v^{3}}\right)(v-w)}{r \cos \theta_{0}\left(1+\frac{3}{u^{4} v^{2}}\right)}
$$




$$
\begin{gathered}
\frac{d v}{d r}=\frac{w-v}{r}, \text { and } \\
\frac{d w}{d r}=\frac{w y^{\prime}}{y}+\frac{y^{2}-w^{2}}{v r}\left\{\frac{\frac{v}{u}-\frac{1}{(u v)^{3}}}{\frac{u}{v}-\frac{1}{(u v)^{3}}}\right\}
\end{gathered}
$$

If a modified form of Hooke's law with

$$
T_{1}=\frac{E h}{1-v^{2}}\left(\varepsilon_{1}+v \varepsilon_{2}\right) \text { and } T_{2}=\frac{E h}{1-v^{2}}\left(\varepsilon_{2}+v \varepsilon_{1}\right)
$$

where $\varepsilon_{1}=u-1$ and $\varepsilon_{2}=v-1$ is used rather than Mooney's model for the constitutive equations in the preceding derivation, one obtains the following equations.

$$
\begin{gathered}
\frac{d y}{d r}=\frac{1}{r \cos \theta_{0}}\left(\frac{w(v-u)(1-v)}{v}+v(v-w)\right) \\
\frac{d v}{d r}=\frac{w-v}{r}, \text { and } \\
\frac{d w}{d r}=\frac{w y^{\prime}}{y}+\frac{y^{2}-w^{2}}{v r}\left(\frac{v-1+v(u-1)}{u-1+v(v-1)}\right)
\end{gathered}
$$

With appropriate boundary conditions, these equations can be numerically integrated to calculate the deformation and shape of the deformed membrane. The Runge-Kutta method is used. The assumed boundary conditions for the membrane include the following: 
1. The membrane is rigidly held at inner radius $r_{0}$ by the capsule and at the outer radius $\mathrm{r}_{2}$ by the hohlraum. This implies that the circumferential stretch $v$ is unity where the membrane attaches to the capsule and to the hohlraum. 2. The meridianal stretch $u$ at the inner radius of the membrane is chosen to be a value which will not exceed the elastic limit of the membrane. The membrane deformation is calculated for a range of $u$ values.

3. For each $\frac{\mathrm{r}_{1}}{\mathrm{r}_{2}}$ and $u$ value we estimate a value for $w=\frac{d \rho}{d r}$, and compute the membrane deformation from $r_{0}$ to $r_{2}$. If the computed circumferential stretch at the outer edge of the membrane $v\left(\mathrm{r}_{2}\right)$ is not equal to one, we have estimated the wrong value of $w$. We try new values of $w$ until one is found which gives $v\left(\mathrm{r}_{2}\right)=1$.

To compute the membrane (and capsule) axial displacement $\eta$, an additional differential equation is needed. Referring to Fig. 3-9, we can deduce the equation

$$
d \eta=-\sqrt{(d s)^{2}-(d \rho)^{2}} \text { or } \frac{d \eta}{d r}=-\sqrt{y^{2}-w^{2}}
$$

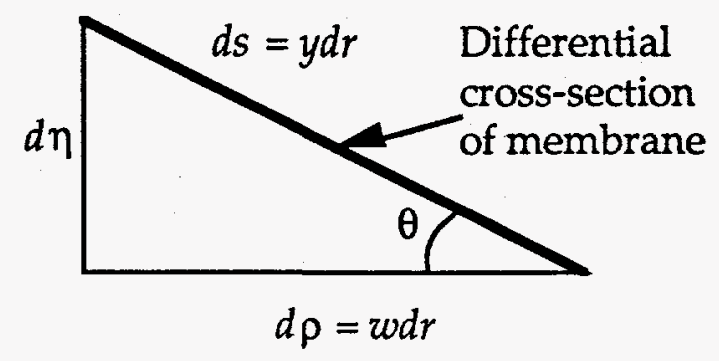

Figure 3-9. Differential cross-section of membrane.

We also see from Fig. 3-9 that the angle $\theta$ of the membrane as it leaves the capsule is given by 


$$
\theta=\left|\arctan \left(\frac{d \eta}{d \rho}\right)\right|=\left|\arctan \left(\frac{\sqrt{y^{2}-w^{2}}}{w}\right)\right|
$$

In doing these computations we are primarily interested in determining two quantities. First we wish to know the displacement $\Delta \eta_{0}=\eta\left(r_{0}\right)-\eta_{u}\left(r_{0}\right)$ as this gives the uncertainty of the capsule position within the target. Second, since net force equals mass times acceleration, we need to know the net force $F$ exerted on the capsule. The net force is given by

$$
\begin{aligned}
F & \approx 2 \pi r_{0} T_{1} \sin \theta \\
& \approx 2 \pi \frac{r_{1}^{2}}{r_{2}} 2 C h\left[\frac{u}{v}-\frac{1}{u^{3} v^{3}}\right] \sin \theta \quad \text { (Mooney's model) } \\
& \approx 2 \pi \frac{r_{1}^{2}}{r_{2}} \frac{h E}{1-v^{2}}[(u-1)+v(v-1)] \sin \theta \quad \text { (Hooke's law) } \\
& \approx 2 \pi \frac{r_{1}^{2}}{r_{2}} \frac{h E \varepsilon_{1}}{1-v^{2}} \sin \theta
\end{aligned}
$$

Equation (3-63) uses assumption \#1 above and neglects the slight reduction in membrane thickness with small strain. So given the capsule and hohlraum radius $\left(r_{1}\right.$ and $\left.r_{2}\right)$, membrane thickness $(h)$, meridianal membrane strain $\left(\varepsilon_{1}\right)$, modulus of elasticity (E), and Poisson's ratio (v), we need only the computed value of $\theta$ to approximate the net force on the capsule. With the capsule mass also known we can then readily compute capsule acceleration by the equation $F=m a$. Figure 3-10 shows the computed values of $\theta$ vs inner membrane strain for various capsule radius to hohlraum radius ratios $\left(r_{1} / r_{2}\right)$. Figure 3-10a used Hooke's law with Poisson's ratio of zero. Figure 3-10b used Mooney's model. Hooke's law with Poisson's ratio of 0.5 (incompressible) gives similar results to Mooney's model. The expected capsule radius to 
hohlraum radius ratio is about 0.4 . As shown in Fig. 3-10a, with zero Poisson's ratio, the angle increases from about 0.41 radians to 0.47 radians as the strain increases from 0 to 0.04 . Somewhat larger angles would result from incompressible materials. The net force is proportional to $\varepsilon_{1} \sin \theta$.
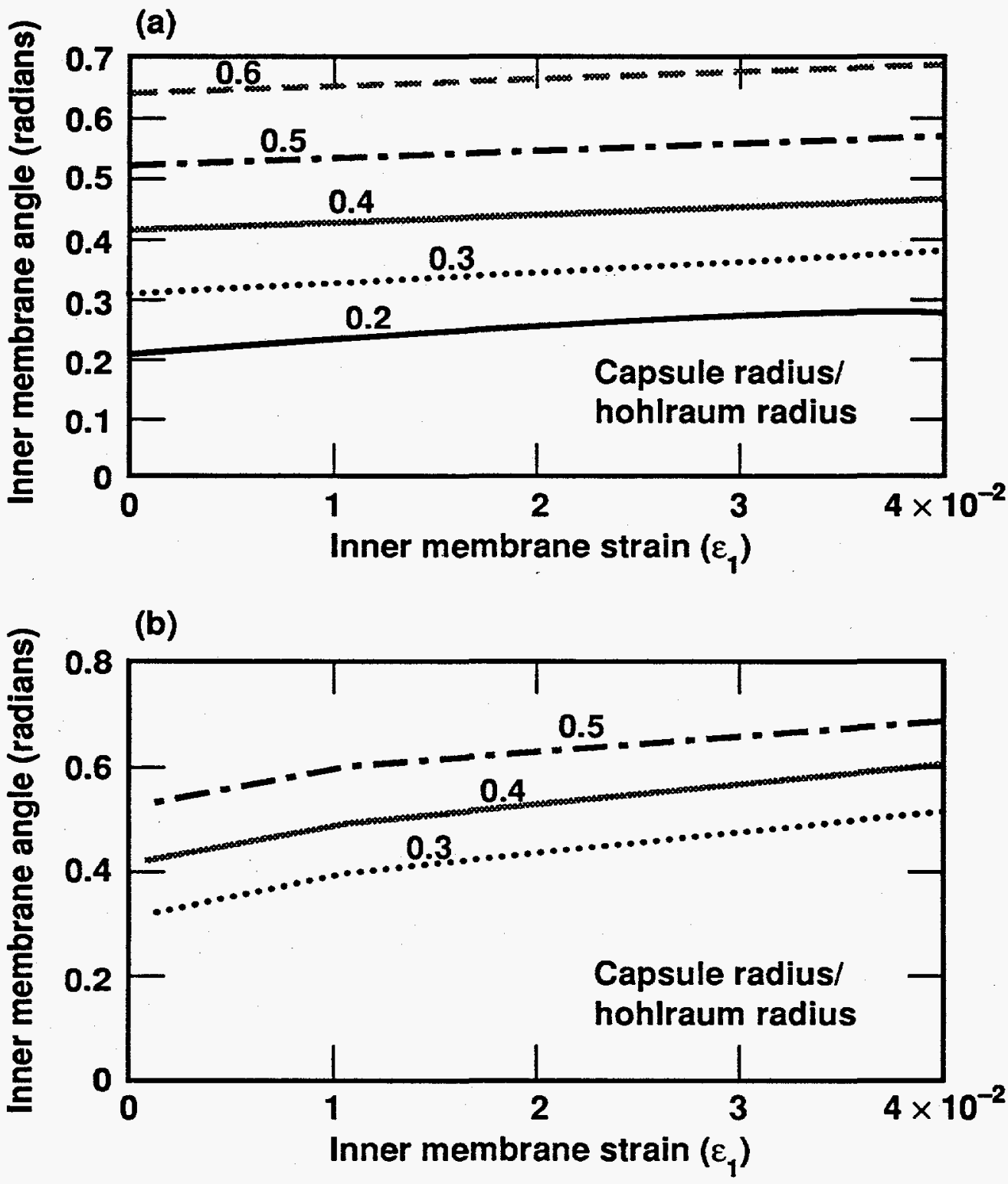

Figure 3-10 (a). The computed angle at which the membrane leaves the capsule vs inner membrane strain for various capsule radius to hohlraum radius ratios $\left(r_{1} / r_{2}\right)$, (Poisson's ratio is 0.0$)$. (b) The computed angle at which 
the membrane leaves the capsule vs inner membrane strain for various capsule radius to hohlraum radius ratios $\left(r_{1} / r_{2}\right)$. Mooney's model was used in the calculations (Equivalent results are obtained using Hooke's law with Poisson's ratio of 0.5 ).

It is also important to know how much the capsule moves relative to the hohlraum as the membrane stretches. This movement causes oscillations of the capsule in the hohlraum and (if these oscillations are not damped or otherwise attenuated) an uncertainty in capsule position. The maximum allowed uncertainty in capsule position relative to the hohlraum is not well known and will require further study. If excessively far off center, the radiation symmetry will be disrupted. We assume that if the displacement is less than about $2 \%$ of the hohlraum radius, the target performance will not be substantially downgraded. A method for attenuating the oscillations will be described shortly. As is shown in Fig. 3-11, the capsule displacement vs membrane strain is quite linear for a range of $r_{1} / r_{2}$ values and is not very sensitive to capsule radius. (Figure 3-11a used Poisson's ratio of 0.0. Figure 3-11b used Mooney's model). With maximum strain of 0.04 , the capsule displacement is about $3-4 \%$ of the hohlraum radius. However, if the displacement is still excessive, the displacement could be reduced by about a factor of two by pre-stressing the two opposing membranes to one half of the maximum stress anticipated. As the capsule is accelerated, the stress and strain of one membrane increases, while the stress and strain of the other membrane decreases. The net force is about the same on the capsule with only half the capsule displacement in the hohlraum. 

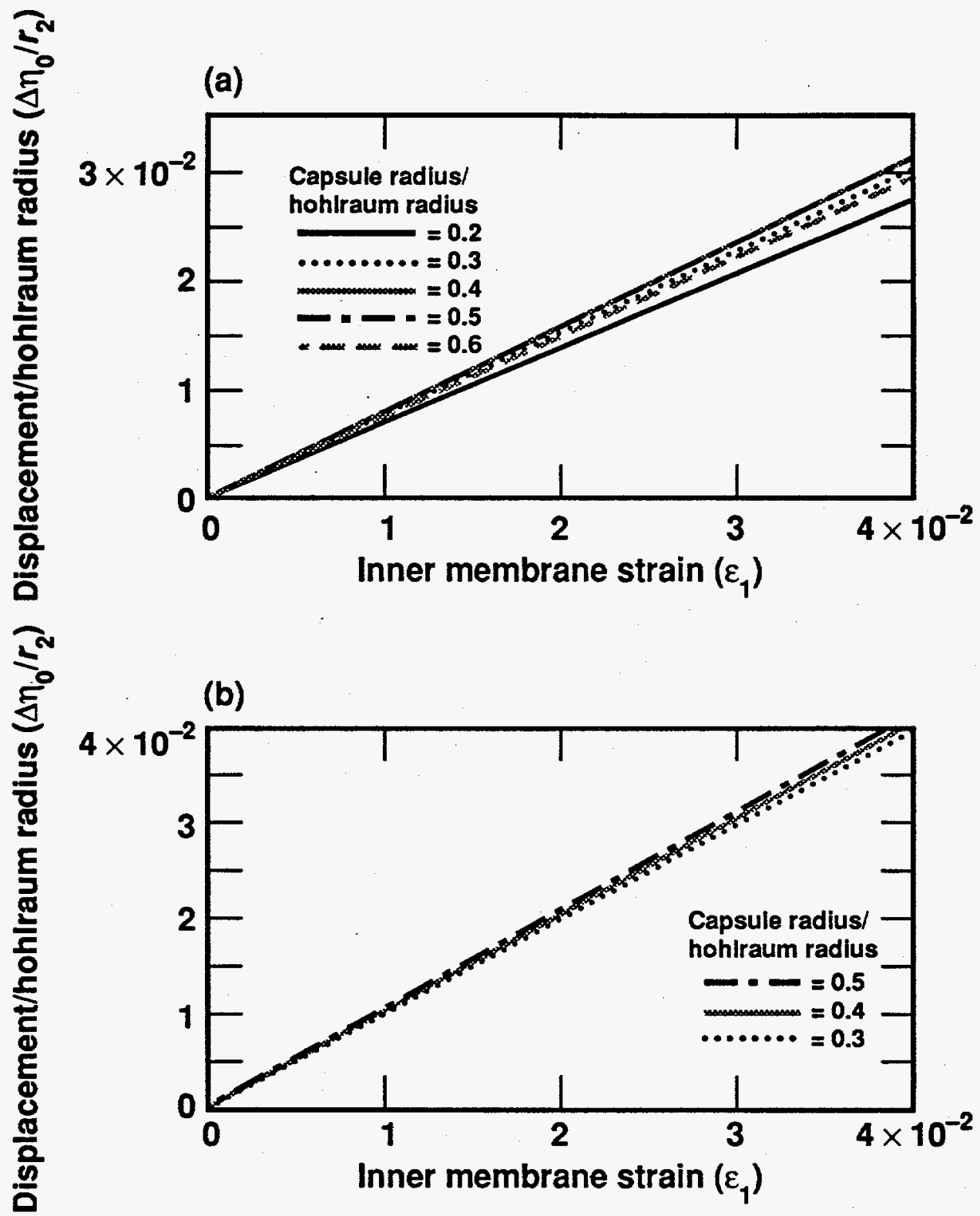

Figure 3-11. (a) Capsule displacement vs inner membrane strain for various capsule radius to hohlraum radius ratios $\left(r_{1} / r_{2}\right)$. (Poisson's ratio is 0.0$)$. (b) Capsule displacement vs inner membrane strain for various capsule radius to hohlraum radius ratios $\left(r_{1} / r_{2}\right)$. Mooney's model was used in the calculations. (Equivalent results are obtained using Hooke's law with Poisson's ratio of 0.5). 
The force exerted by dual membranes on a capsule is much greater and the capsule displacement is much less than for single membranes that were covered in reference 6 .

The acceleration must be large enough to meet the reaction chamber pulse rate requirements. The membrane must be thin enough to not adversely effect target implosion symmetry. As indicated in Fig. 3-12, the dual membrane configuration has a better opportunity of satisfying both criteria.

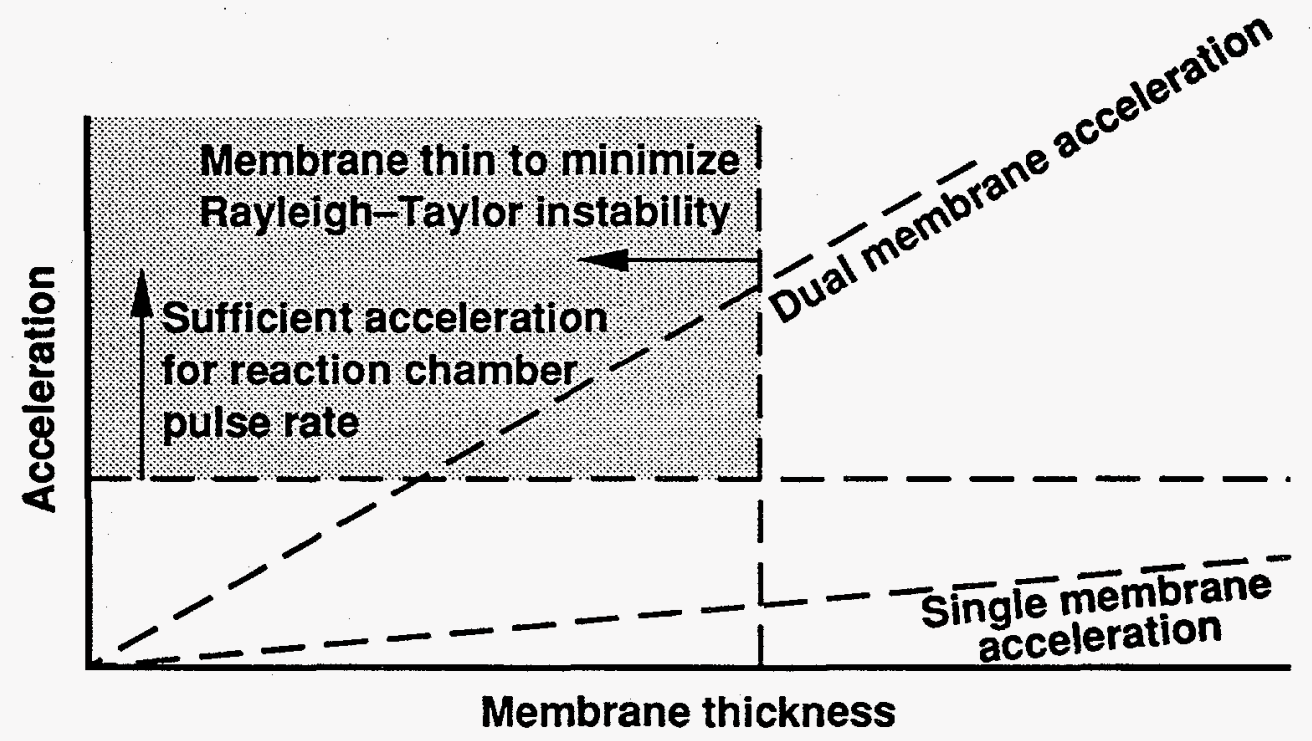

Figure 3-12. The target acceleration must be large enough to achieve the reaction chamber pulse rate. However, the membrane must be very thin so that energy may be absorbed uniformly on the capsule surface allowing the capsule to implode more uniformly to the capsule center. The available operating range is shaded. These constraints are more easily satisfied with a dual membrane design. 


\section{Example 3.4-1.}

Assume the following typical parameters for a dual membrane supported capsule.

Capsule radius $=\mathrm{r}_{1}=2.4 \mathrm{~mm}$

Hohlraum radius $=r_{2}=6 \mathrm{~mm}$

Membrane strain $=\varepsilon_{1}=0.02$

Membrane thickness $=h=10^{-7} \mathrm{~m}$

Capsule mass $=\mathrm{m}=30 \mathrm{mg}$

Material $=$ cryogenic mylar

Determine the capsule displacement and the capsule acceleration using the linear model and the non-linear model with both Hooke's law with $v=0$ and Mooney's model material assumptions.

The capsule displacement $\Delta \eta_{o}$ is obtained from figures 3-7 and 3-11 using $\mathrm{r}_{1} / \mathrm{r}_{2}=0.4$.

$$
\text { Linear } \quad \Delta \eta_{0}=0.8\left(\mathrm{r}_{2}\right)\left(\varepsilon_{1}\right)=96 \mu \mathrm{m}
$$

Hooke's Law $\Delta \eta_{0}=0.015\left(\mathrm{r}_{2}\right)=90 \mu \mathrm{m}$

Mooney's $\quad \Delta \eta_{o}=0.02\left(\mathrm{r}_{2}\right)=120 \mu \mathrm{m}$

$\Delta \eta_{o}$ is $<2 \%$ of the hohlraum radius. If this displacement is unacceptable, $\Delta \eta_{o}$ can be reduced to about 50-60 $\mu \mathrm{m}$ by prestressing the membranes. A method to further reduce oscillations will be discussed in section 3.5.

The capsule acceleration is obtained from equations (3-63) and $F=m a$. With the linear theory the increase in the membrane angle $\theta$ was neglected resulting in a slightly lower calculated acceleration. 


$$
\begin{aligned}
a & =\frac{F}{m} \approx \frac{2 \pi}{m} \frac{r_{1}^{2}}{r_{2}} \frac{h E \varepsilon_{1}}{1-\mu^{2}} \sin \theta \\
& =\frac{6.28}{3 \times 10^{-5} \mathrm{~kg}} \frac{\left(2.4 \times 10^{-3} \mathrm{~m}\right)^{2}}{6 \times 10^{-3} \mathrm{~m}} \frac{10^{-7} \mathrm{~m}\left(7 \times 10^{9} \mathrm{~Pa}\right)(0.02)}{1-0} \frac{2.4}{6} \\
& =1125 \mathrm{~m} / \mathrm{s}^{2}
\end{aligned}
$$

With Hooke's law we use Fig. 3-10a for membrane angle.

$$
\begin{aligned}
a & =\frac{F}{m} \approx \frac{2 \pi}{m} \frac{r_{1}^{2}}{r_{2}} \frac{h E \varepsilon_{1}}{1-\mu^{2}} \sin \theta \\
& =\frac{6.28}{3 \times 10^{-5} \mathrm{~kg}} \frac{\left(2.4 \times 10^{-3} \mathrm{~m}\right)^{2}}{6 \times 10^{-3} \mathrm{~m}} \frac{10^{-7} \mathrm{~m}\left(7 \times 10^{9} \mathrm{~Pa}\right)(0.02)}{1-0^{2}} \sin (0.44 \mathrm{rad}) \\
& =1198 \mathrm{~m} / \mathrm{s}^{2}
\end{aligned}
$$

With Mooney's model we use Fig. 3-10b for the membrane angle.

$$
\begin{aligned}
a & =\frac{F}{m} \approx \frac{2 \pi}{m} \frac{r_{1}^{2}}{r_{2}} 2 \operatorname{Ch}\left(\frac{u}{v}-\frac{1}{u^{3} v^{3}}\right) \sin \theta \\
& =\frac{2 \pi}{m} \frac{r_{1}^{2}}{r_{2}} \frac{h E}{3}\left(u+u^{-3}\right) \sin \theta \\
& =\frac{6.28}{3 \times 10^{-5} \mathrm{~kg}} \frac{\left(2.4 \times 10^{-3} \mathrm{~m}\right)^{2}}{6 \times 10^{-3} \mathrm{~m}} \frac{10^{-7} \mathrm{~m}\left(7 \times 10^{9} \mathrm{~Pa}\right)}{3}\left(1.02-1.02^{-3}\right) \sin (0.53 \mathrm{rad}) \\
& =1840 \mathrm{~m} / \mathrm{s}^{2}
\end{aligned}
$$

As shown in Fig. 3-13, the membrane-hohlraum boundary should be designed to avoid a sharp radius of curvature $R$ at the boundary. The approximate additional strain on the membrane due to bending is given by $\varepsilon=\frac{h}{2 R}$. $R$ must be made large enough that the bending strain plus the stretching strain does not cause the membrane to break at the boundary. 


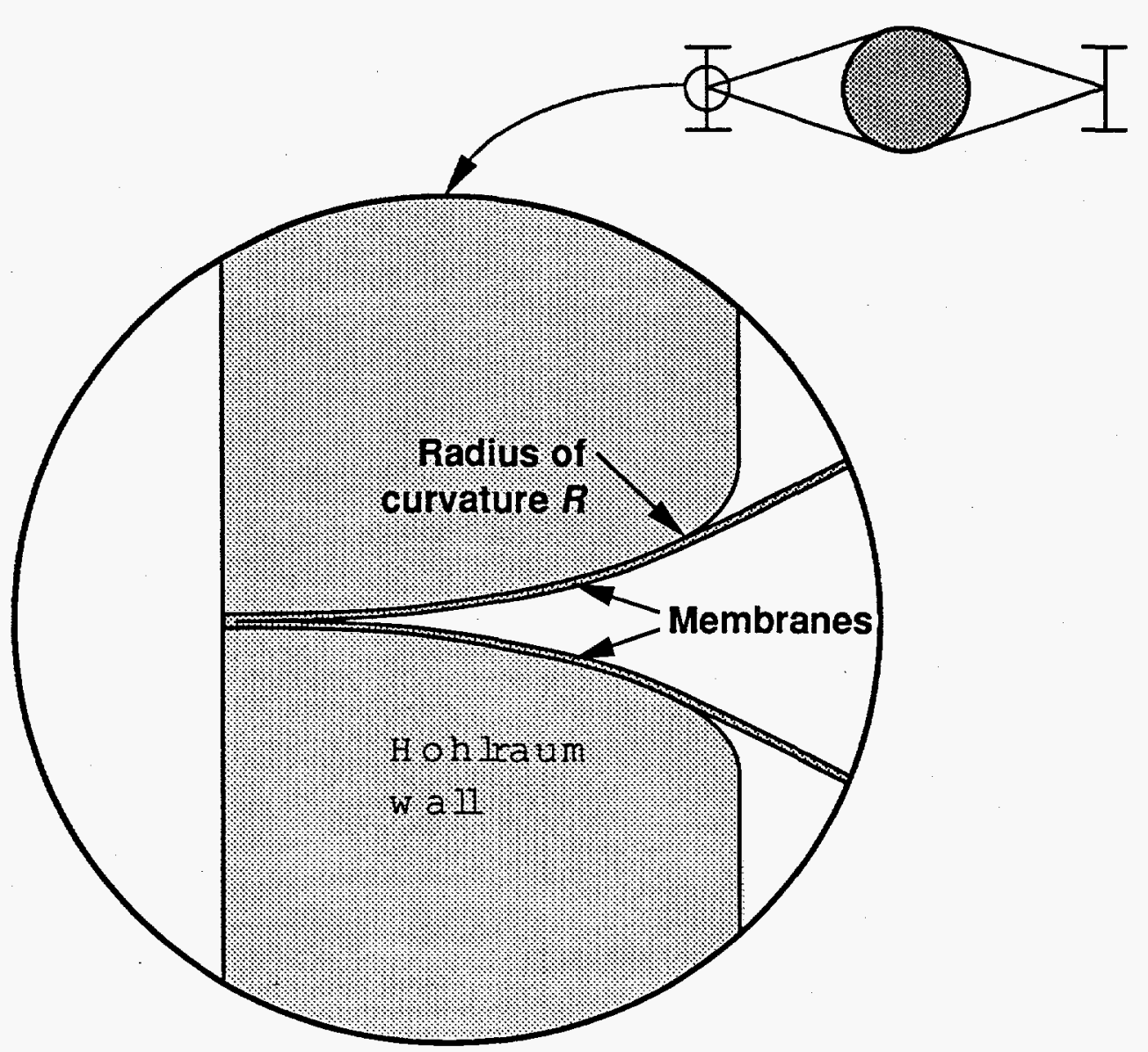

Figure 3-13. Interface between membrane and hohlraum is rounded to minimize membrane strain.

\subsection{Effects of Membrane Vibration}

Since the capsule and hohlraum have much larger mass than the membrane, the large amplitude membrane vibrations will be due to oscillations of the capsule within the hohlraum. The smaller superimposed vibrations of the membrane (similar to those of a drum head) will be neglected here.

Membrane vibrations (capsule oscillations within hohlraum) could have several adverse effects. Vibrations will increase the maximum instantaneous acceleration of the capsule directly increasing the maximum stress and strain 
of the membrane. Vibrations may increase the uncertainty of the capsule position. Vibrations may cause fatigue failure of the membrane. Vibrations may couple with other target motions, causing the target to tumble.

As will be shown, the vibrations can be greatly reduced by a well selected acceleration profile. Additionally, the adverse effects of vibrations would be reduced if vibration damping were present. Membranes with damping characteristics would be preferred. The remaining paragraphs in this section assume no damping is present.

\subsubsection{Vibration Frequency}

As noted from Fig. 3-11, the capsule displacement is directly proportional to the membrane strain. If we neglect the rather small membrane angle increase with small strain, the force on the capsule is nearly proportional to the capsule displacement $\left(F \approx k \Delta \eta_{0}\right)$. In this way we can apply the well known relationships for a harmonic oscillator to the motion of the capsule in the hohlraum. For very small capsules in large hohlraums, this would not be correct. A typical value for $\mathrm{k}$ can be obtained using the Mooney's model parameters from example 3.4-1.

$$
\begin{aligned}
& F=m a \approx k \Delta \eta_{0} \\
& \Rightarrow k \approx \frac{m a}{\Delta \eta_{0}} \sim \frac{3 \times 10^{-5} \mathrm{~kg}\left(1840 \mathrm{~m} / \mathrm{s}^{2}\right)}{1.2 \times 10^{-4} \mathrm{~m}} \approx 460 \mathrm{~N} / \mathrm{m}
\end{aligned}
$$

Normally, the capsule mass is much less than the hohlraum mass so that reduced mass is approximately equal to capsule mass. We can approximate the frequency of vibration by the well known vibrational frequency of a harmonic oscillator. 


$$
f=\frac{\omega}{2 \pi}=\frac{1}{2 \pi} \sqrt{\frac{\mathrm{k}}{m}} \sim \frac{1}{6.28} \sqrt{\frac{460 \mathrm{~N} / \mathrm{m}}{3 \times 10^{-5} \mathrm{~kg}}} \sim 0.6 \mathrm{kHz}
$$

\subsubsection{Vibration Amplitude and Resulting Position Uncertainty}

Capsule displacement is nearly proportional to membrane strain. The membrane displacement during target acceleration is a non-linear superposition of a steady capsule acceleration component and a vibrational component. Assuming a linear force vs displacement relationship and that the vibrations are not resonantly driven, the maximum capsule displacement is twice the equilibrium displacement. ${ }^{1}$ Since membrane strain and net force are also nearly proportional to displacement, they would also double.

This would reduce the allowed acceleration by a factor of two. However, this effect could be mitigated if the acceleration increased to maximum value in a controlled manner. For example, an effective method to minimize the vibrations is to increase the acceleration gradually over a few vibration cycles (i.e. a few ms). We computed the results of gradually increasing and decreasing acceleration. We used dimensionless parameters and Hooke's law, assuming a constant value for the spring constant $k$. For example, Fig. 3-14 shows the capsule displacement in the hohlraum vs time for the assumption that the hohlraum acceleration is increased over a time period of 2.5 oscillation cycles, held constant for the next 7 cycles and decreased over the next 2.5 cycles. The figure indicates that the oscillatory overshoot during the capsule acceleration is reduced from $100 \%$ to about $13 \%$. The final oscillations are reduced from $200 \%$ of the equilibrium acceleration position to $25 \%$. This reduction in final oscillations is important because membrane vibrations

\footnotetext{
${ }^{1}$ Typical values for the equilibrium displacement strain and force were calculated in section 3.4.
} 
cause position deviations of the capsule inside the hohlraum. The hohlraum also vibrates about the center of mass of the target and causes uncertainty in the measurement of target position. Since the mass of the hohlraum is much greater than the mass of the capsule, the target position uncertainty is much less than the capsule position uncertainty.

The 2.5 cycle rise time indicated in Fig. 3-14 is far from optimum. Figure 3-15 shows maximum normalized capsule displacement as a function of acceleration rise time. The oscillatory 'overshoot' is zero for acceleration rise times equal to integral numbers of cycles and reaches local maximums near integer plus half cycle rise times.

The choice of decreasing the rise after 9.5 cycles as indicated in Fig. 3-14 is also far from optimum. Figure 3-16 shows the residual oscillation magnitude after the acceleration is decreased as a function of when the acceleration reduction began. The lower curve assumes the oscillations rise and fall gradually over 2.5 cycles. The upper curve assumes instantaneous application and removal of acceleration. The oscillations resulting from abrupt acceleration application are about 8 times larger. In either case, if the reduction begins after an integral number of cycles, there are no residual oscillations. The residual oscillations are maximized if the reduction begins in the middle of a cycle.

We conclude that an order of magnitude reduction in the vibrations is readily achievable by ramping the acceleration up and back down over a few target vibration periods. 


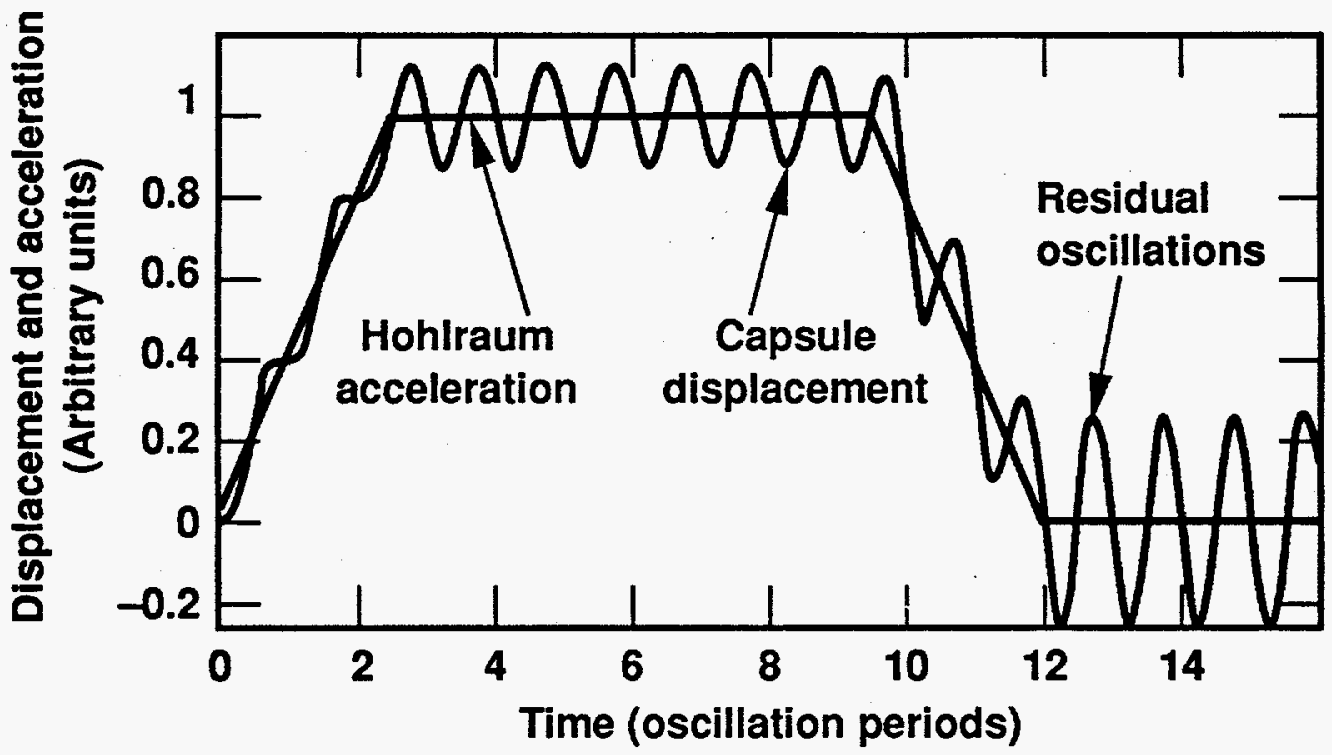

Figure 3-14. Normalized hohlraum acceleration and axial capsule displacement from center of hohlraum. This graph assumes that the force on the capsule is proportional to the capsule displacement from the center. Here, the acceleration increases and later decreases over a period of 2.5 cycles. By comparison, if the acceleration were to increase and decrease abruptly, the initial oscillations would be between 0 and 2 and the post accelerations worst case oscillations would be between 2 and -2 . 


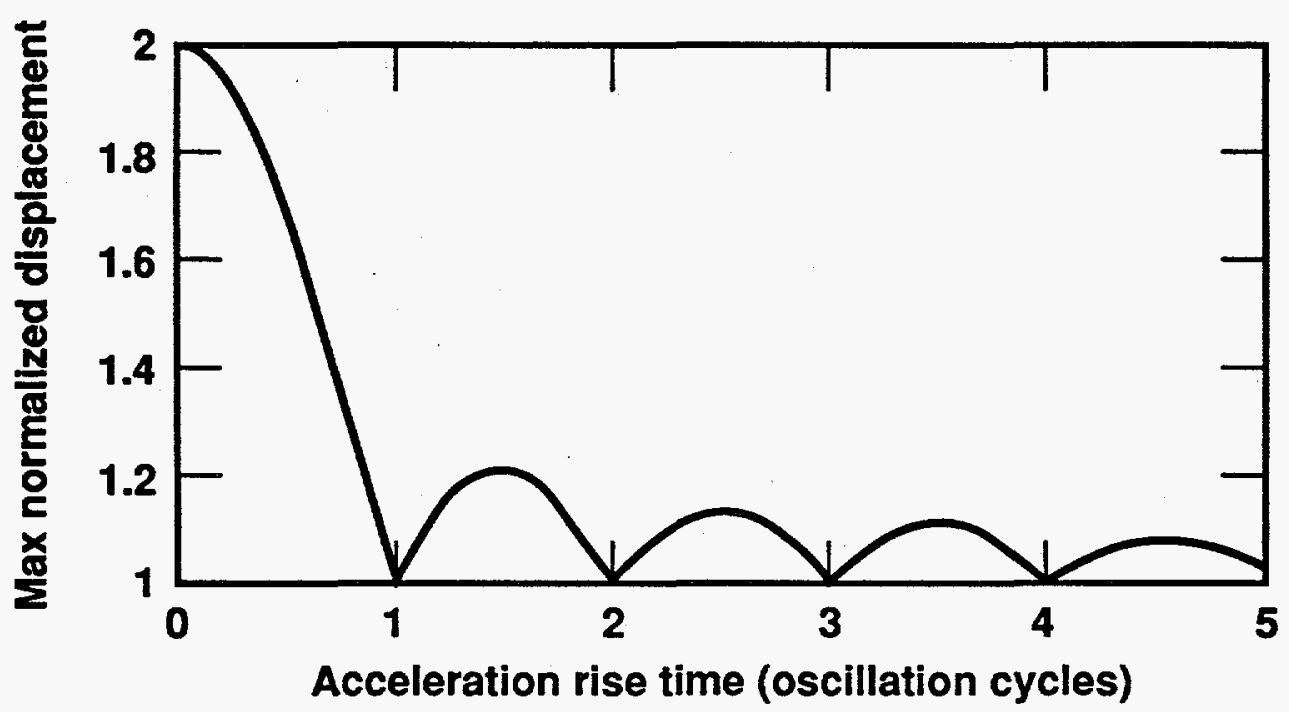

Figure 3-15. Maximum normalized capsule displacement as a function of acceleration rise time. The acceleration is assumed to increase at a uniform rate until the maximum acceleration is reached. A maximum displacement of 1.0 indicates zero capsule oscillations during the constant part of the acceleration. Typically, an order of magnitude reduction in the capsule displacement "overshoot" is possible by increasing the acceleration gradually over a few oscillation time periods. 


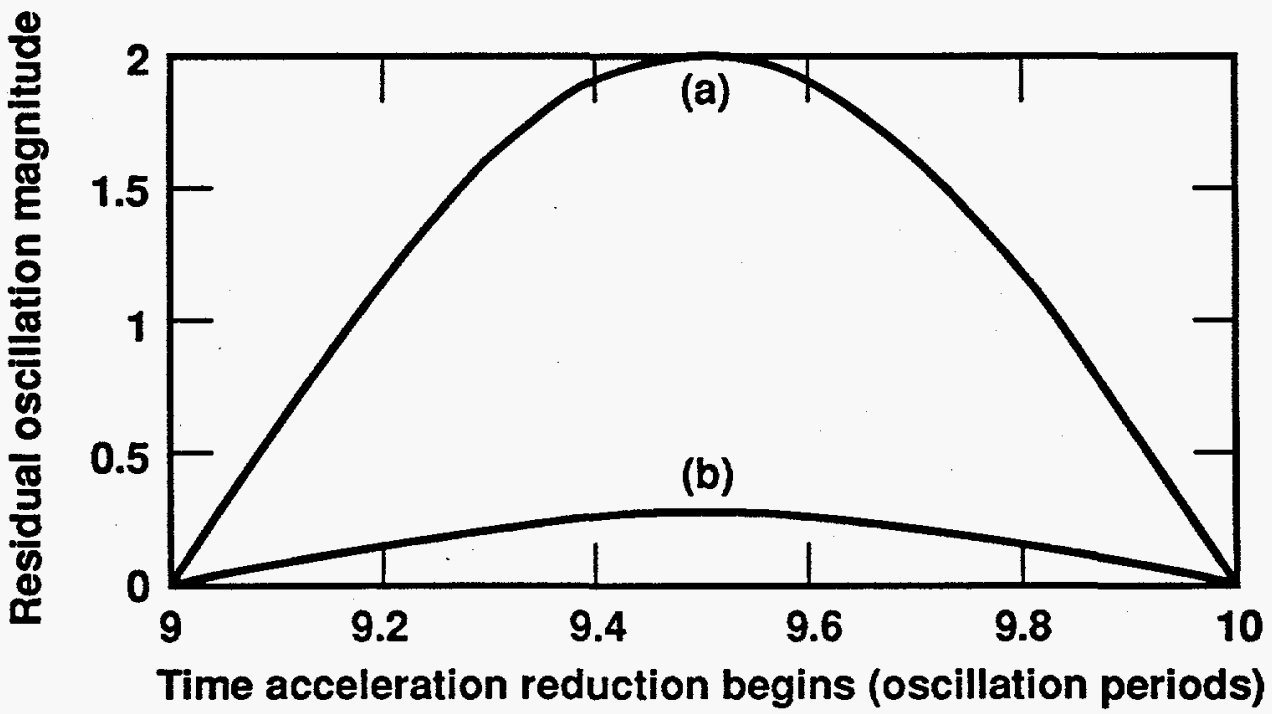

Figure 3-16. Normalized residual capsule oscillation magnitude depends on when the hohlraum acceleration reduction begins, as well as how rapidly the acceleration is applied. (a) Assumes instantaneous acceleration rise and fall times. (b) Assumes the acceleration rise and fall times are each 2.5 natural oscillation periods.

Hohlraum designs normally require that an axis of symmetry be maintained stably oriented in space. It may be necessary to spin the target about this axis for gyroscopic stability. If the vibration frequency and the rotational frequency are nearly equal or integral multiples of each other a coupling could occur between the two motions. This coupling could cause amplified vibrations or rotational instabilities. 


\subsection{References}

1. D. D. Ho, J. A. Harte, and M. Tabak, "Radiation-Driven Targets for Heavy Ion Fusion," Presented at the Fifteenth International Conference on Plasma Physics and Controlled Nuclear Fusion Research, Seville, Spain, Sep. 26 - Oct. 2, 1994, IAEA-CN-60/B-P-13, to be published in Nuclear Fusion.

2. R. E, Schramm, A. F. Clark, and R. P. Reed, A Compilation and Evaluation of Mechanical, Thermal, and Electrical Properties of Selected Polymers, NBS Monograph 132 (National Bureau of Standards, Boulder, Colorado, 1973), p. 468.

3. S. Timoshenko and S. Woinowski-Krieger, Theory of Plates and Shells, 2nd ed. (McGraw Hill, New York, 1959), pp. 429-465.

4. W. H. Yang and W. W. Feng, "On Axisymmetrical Deformations of Nonlinear Membranes, "Transactions of the ASME, Series E, Journal of Applied Mechanics, 37, 1002 (1970).

5. A. E. Green and J. E. Adkins, Large Elastic Deformations, 2nd ed. (Oxford Univ. Press, London, England, 1970), p. 298.

6. R. W. Petzoldt and R. W. Moir, Membrane Support of Accelerated Fuel Capsules for Inertial Fusion Energy Reactors, Lawrence Livermore National Laboratory, Livermore, CA, UCRL-ID-114020 (1993). 


\section{Chapter 4 - Fuel Stress}

\subsection{Problem Definition and Solution Method}

We used a finite-element code NIKE2D to calculate the stresses that would occur in an IFE target to determine whether DT would be damaged (inelastically deformed) by capsule acceleration. ${ }^{1}$ We used the conservative assumption that the fuel may slide along the interior surface of the capsule without friction or adhesion. The fuel is also assumed to be free to pull away from the capsule without adhesion.

The target model used is shown in Fig. 4-1, and is based on Darwin Ho's design. ${ }^{2}$ The capsule has a $2.34 \mathrm{~mm}$ radius and contains about $3.6 \mathrm{mg}$ of DT fuel and $15 \mathrm{mg}$ of plastic capsule ablator. ${ }^{1}$ The membrane is one micron thick. $^{2}$ An axisymmetric geometry is assumed. To account for the acceleration, a force per unit volume equal to the acceleration times the density and opposite in direction is placed on the target holding the outer edge of the membrane fixed. This flattens the capsule slightly, stretches the lower membrane, and the upper membrane becomes slack. The upper membrane was neglected in these calculations.

\footnotetext{
1 The fuel stress calculated is not very sensitive to which material the capsule is made out of (plastic or beryllium). As long as Young's modulus for the capsule is very much greater than Young's modulus for the DT, the capsule does not deform much and acts almost as a rigid restraint for the DT. The resulting DT stress is a little lower with beryllium which has a much higher Young's modulus than plastic.

2This membrane is thicker than the reference case membrane so that we can test the fuel stress at a larger than reference case acceleration of $10,000 \mathrm{~m} / \mathrm{s}^{2}$. (Membrane stress is discussed in more detail in chapter 3.).
} 


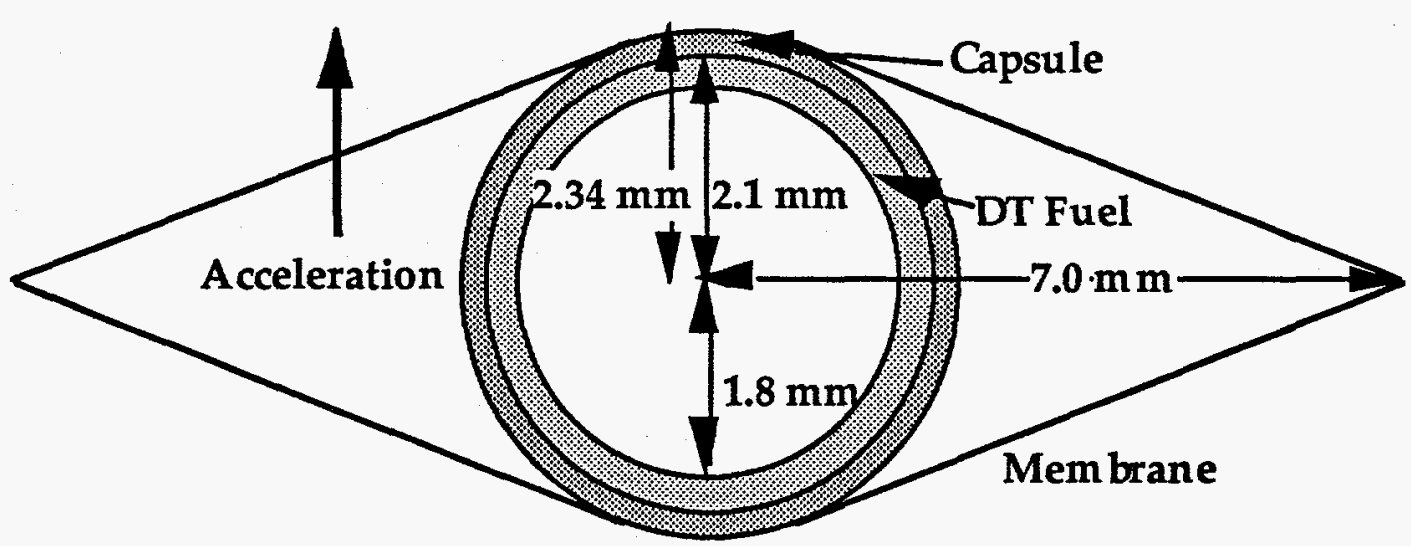

Figure 4-1. A $2.34 \mathrm{~mm}$ radius fuel capsule is accelerated with a $1 \mu \mathrm{m}$ thick membrane.

The fuel was divided into 400 elements $(100 \times 4)$, the capsule into 200 elements $(100 \times 2)$. The membrane is divided into 100 elements $(100 \times 1)$. The problem is done in cylindrical $(\mathrm{r}, \mathrm{z})$ coordinates. The boundary conditions include constant $r$ and $z$ at the outer edge of the membrane and $r=0$ "roller" boundary conditions for the center of the fuel, capsule, and membrane. The membrane is free to slide on and pull away from the capsule surface. The fuel is free to slide on and pull away from the capsule. The membrane and capsule are assumed to be an elastic material with density $1000 \mathrm{~kg} / \mathrm{m}^{3}$, Young's modulus $7 \mathrm{GPa}$ and Poisson's ratio 0.4 . These are approximately the properties of cryogenic mylar as shown in Fig. 3-4. The fuel has density of 250 $\mathrm{kg} / \mathrm{m}^{3}$, Young's modulus of $40 \mathrm{MPa}$, and Poisson's ratio of 0.3 . These are the density of frozen DT and the Young's modulus and Poison's ratio for $D_{2}$ at 16.4 $\mathrm{K}$ (all hydrogen properties taken from reference 3). Young's modulus has not been measured for DT, but is probably higher for a given temperature than $D_{2}$ (we estimate that they are roughly the same with DT at 1 degree higher temperature), just as Young's modulus for $D_{2}$ is much higher than that for $\mathrm{H}_{2}$ (see table 2-4). 


\subsection{Computational Results}

With assumed acceleration of $10,000 \mathrm{~m} / \mathrm{s}^{2}$, the Von Mises (or effective) stress in the fuel is shown in Fig. 4-2.3 The maximum effective stress is only $11 \mathrm{kPa}$. If the fuel adhered to the capsule, the maximum stress is much lower. By comparison, the one dimensional stress $\sigma$ required to accelerate fuel of shell thickness $\delta=0.3 \mathrm{~mm}$ given by

$$
\sigma=\rho \delta a=250 \mathrm{~kg} / \mathrm{m}^{3}\left(3 \times 10^{-4} \mathrm{~m}\right)\left(10^{4} \mathrm{~m} / \mathrm{s}^{2}\right)=0.75 \mathrm{kPa} .
$$

The resulting stress increases almost linearly with increased acceleration. As long as the Von Mises stress remains below the yield stress, the fuel should not be damaged. Yield stress for DT is not known but must be at least as high as yield stress for $D_{2}$ at a given temperature. Experiments to determine the yield stress and Young's modulus for DT as a function of temperature would be very useful for this study. Young's modulus and the yield stress of hydrogen isotopes depend strongly on temperature, particularly as the temperature approaches the triple point. Yield stress for $\mathrm{D}_{2}$ is $50 \mathrm{kPa}$ at $16.4 \mathrm{~K}$ and $80 \mathrm{kPa}$ at $15.6 \mathrm{~K}$. DT has a melting point about $1 \mathrm{~K}$ higher than $\mathrm{D}_{2}(19.7$ K). ${ }^{2}$ As noted in section 2.6 , it is reasonable to expect that for DT temperatures less than $17.4 \mathrm{~K}$, the yield stress will be greater than $50 \mathrm{kPa}$. We assumed in chapter 2 that a higher final fuel temperature may be acceptable, but this is in the reaction chamber after the target acceleration has been completed. This is almost 5 times higher than the maximum effective stress calculated for a $10,000 \mathrm{~m} / \mathrm{s}^{2}$ acceleration. Acceleration of $10,000 \mathrm{~m} / \mathrm{s}^{2}$ should be possible with a

\footnotetext{
${ }^{3}$ This is 5 times higher than the reference case acceleration of $2,000 \mathrm{~m} / \mathrm{s}^{2}$. The resulting stresses are reduced almost linearly with less acceleration.
} 
safety factor of about 5 below yield stress of the DT. Of course, lower acceleration such as our reference case acceleration of $2,000 \mathrm{~m} / \mathrm{s}^{2}$ is also possible. This allowed acceleration is nearly proportional to the yield stress of DT which will strongly depend on temperature.

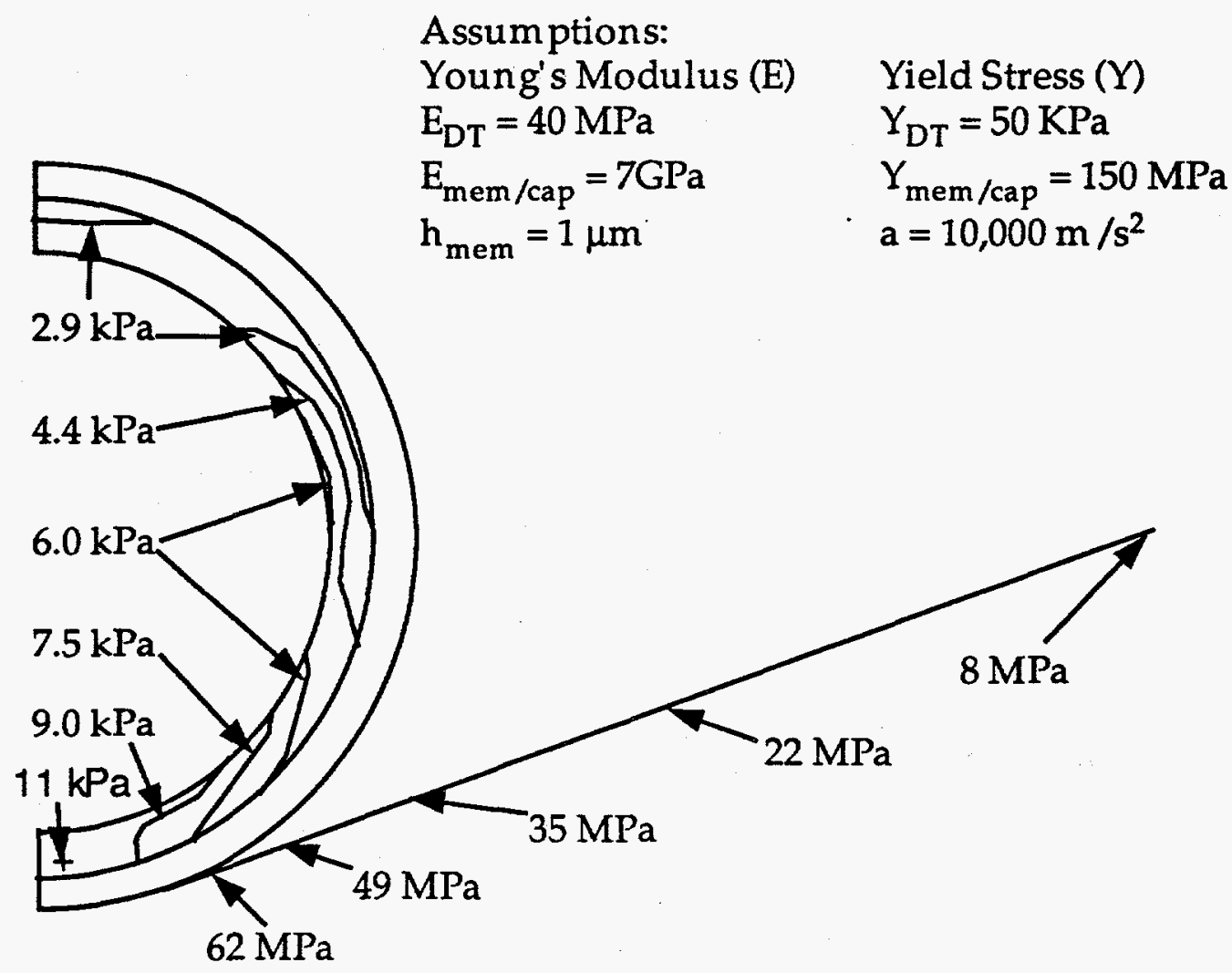

Figure 4-2. Stresses in fuel and membrane for an accelerated target.

The membrane stress and strain are also well below the failure stress and strain of cryogenic mylar. However, we chose membrane strain a membrane strain 10 times thicker than our reference case $0.1 \mu \mathrm{m}$ to allow for this higher acceleration.

According to calculations, a typical target can undergo acceleration of greater than $10,000 \mathrm{~m} / \mathrm{s}^{2}$ without damage to the fuel if the fuel temperature is below $17 \mathrm{~K}$. Experimental evaluation of DT Young's modulus and yield stress 
as a function of temperature would be useful to more precisely quantify this result.

\subsection{References}

1. Bruce Englemann, NIKE2D A Nonlinear, Implicit, Two-Dimensional Finite Element Code for Solid Mechanics - User Manual, Lawrence Livermore National Laboratory, Livermore, CA, UCRL-MA-105413 (1991).

2. D. D. Ho, J. A. Harte, and M. Tabak, "Radiation-Driven Targets for Heavy Ion Fusion," Presented at the Fifteenth International Conference on Plasma Physics and Controlled Nuclear Fusion Research, Seville, Spain, Sep. 26 - Oct. 2, 1994, IAEA-CN-60/B-P-13, to be published in Nuclear Fusion.

3. P. C. Souers, Hydrogen Properties for Fusion Energy, (University of California Press, Berkeley, CA, 1986), pp. 78-86. 


\section{Chapter 5 - Target Steering}

\subsection{Introduction}

Target injectors might be capable of meeting the required $\pm 0.2 \mathrm{~mm}$ accuracy discussed in section 2.3 , but probably will not. If the target tracking measurements indicate the beams will not hit the target with the required accuracy, the beams must be redirected to the target or the target must be steered to the beams. Since there is only one target and many beams, it might be simpler to steer the target than to actively point the beams.

The time that the target will pass the beam focal point is predicted based on target tracking measurements. The driver beam timing is then adjusted to arrive simultaneously with the target, thus avoiding the need to correct the target's position along its direction of motion. Steering the target perpendicular to its motion is possible, avoiding the need to steer the beams.

Potential methods of steering a target include giving the target an electrical charge and passing it through an electric field, exerting an impulse on the target with a puff of gas or other material, or using ablation from the side of a target caused by laser heating for example.

The electric field method of target steering is discussed in this chapter. We find that electric fields acting on charged targets can provide more steering than is likely to be required without excessively increasing target tumble angle.

\subsection{Electrostatic Steering}

At normal injection speeds, the force that can be exerted on a charged target with electric fields is orders of magnitude stronger than the force that can be exerted by magnetic fields. 
As indicated in Fig. 5-1, the target with charge $q$, mass $m$, and speed $v$ passes between target steering electrodes with potential difference $V$, length $L_{S}$, and spacing $Y_{p}$. The electric field strength between the electrodes is approximately given by $E=V / Y_{p}$. The target subsequently travels an extra distance $L_{c}$ toward the focal point near the reaction chamber center. The target steering displacement $\Delta Y$ is given by

$$
\begin{aligned}
\Delta Y & =\int_{0}^{t_{s}+t_{c}} v_{y}(t) d t \\
& =\int_{0}^{t_{s}} a t d t+\int_{t_{s}}^{t_{s}+t_{c}} a t_{s} d t \\
& =\frac{E q}{m}\left\{\frac{t^{2}}{2}\left|\begin{array}{l|l}
\frac{L_{s}}{v} \\
0
\end{array}+\frac{L_{s} t}{v}\right| \begin{array}{c}
\frac{L_{s}+L_{c}}{v} \\
\frac{L_{s}}{v}
\end{array}\right\} \\
& \approx \frac{V q}{Y_{p} m v^{2}}\left\{\frac{L_{s}^{2}}{2}+L_{s} L_{c}\right\}
\end{aligned}
$$

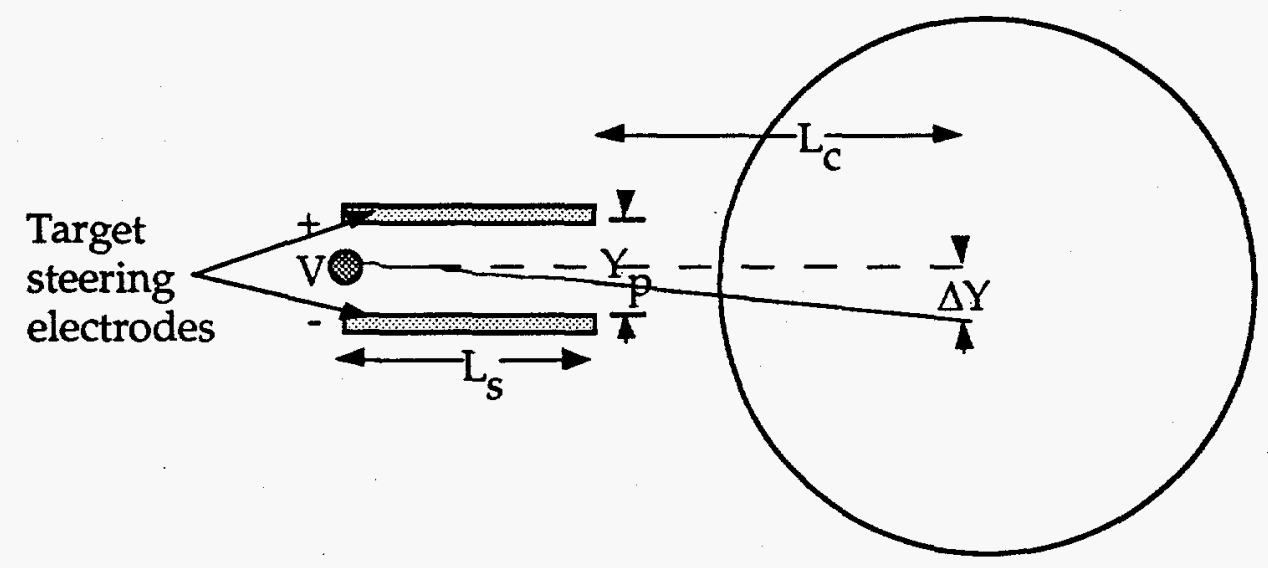

Figure 5-1. The charged target is steered by an electric field between the target steering electrodes.

The value of $E=V / Y_{p}$ is limited by vacuum breakdown sparking. When the target is between the electrodes, the fields and potentials of the target and electrodes interact affecting each others values. Later in this chapter this interaction is investigated with a three dimensional computational model. For 
now we will investigate the independent fields and potentials of the target and electrodes.

Kilpatrick's criterion ${ }^{1}$ which states that if

$$
V E^{2}\left\{\exp \left(\frac{-1.7 \times 10^{7}}{E}\right)\right\} \leq 1.8 \times 10^{18}
$$

where $E$ is the cathode (negative electrode) electric field, then vacuum sparking should not occur ( $E$ and $V$ are in SI units). This criterion allows rather strong electric fields which are weakly dependent on the electrode spacing $Y_{p}$. As shown in Fig. 5-2, the maximum electric field drops from $10 \mathrm{MV} / \mathrm{m}$ down to 6.3 $\mathrm{MV} / \mathrm{m}$ as the electrode spacing varies an order of magnitude from $1 \mathrm{~cm}$ to $10 \mathrm{~cm}$. The maximum potential difference between the electrodes increases from $100 \mathrm{kV}$ to $600 \mathrm{kV}$ over the same range.

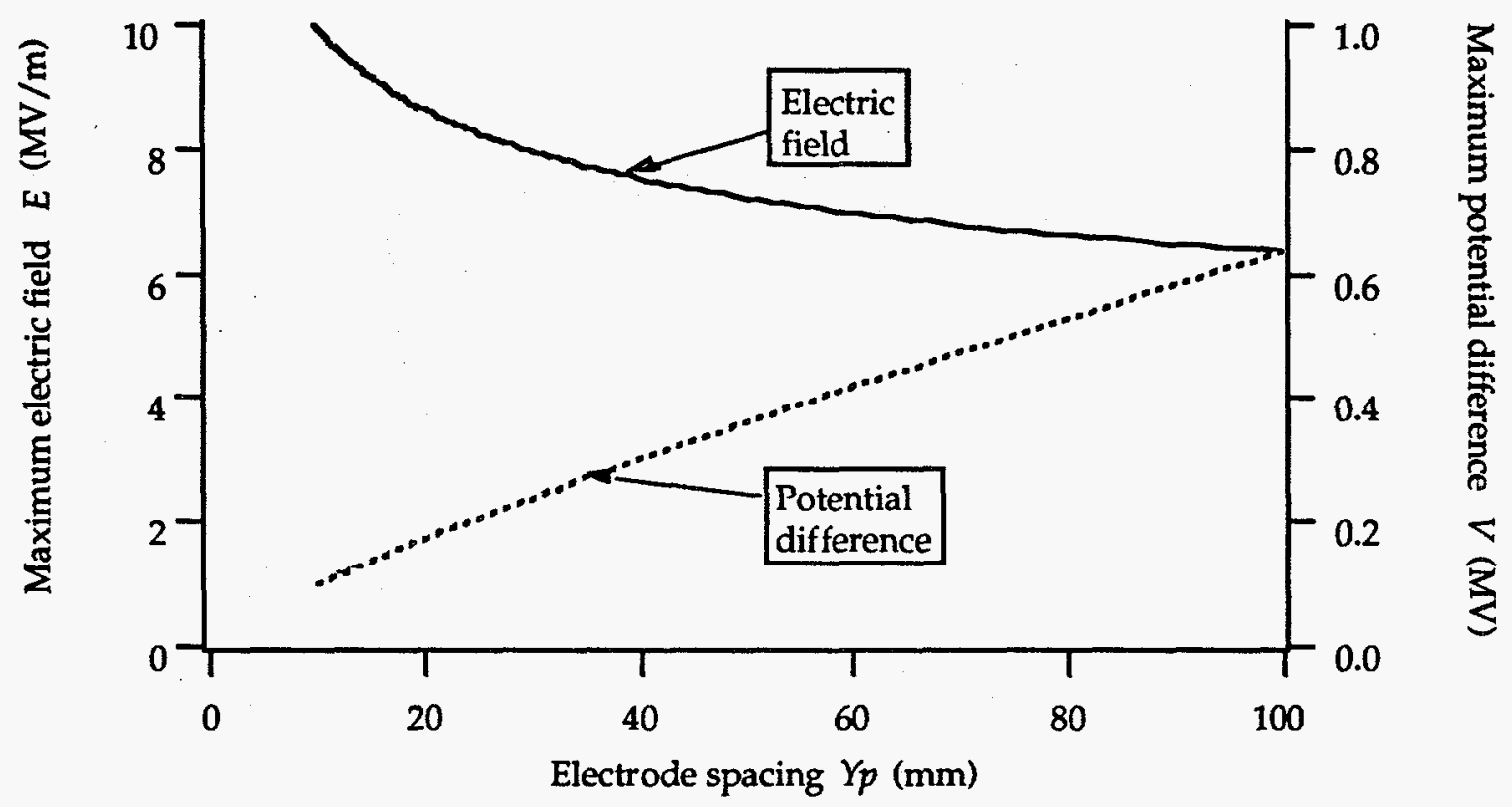

Figure 5-2. An electric field of more than $6 \mathrm{MV} / \mathrm{m}$ will not cause sparking between steering electrodes over the range of electrode spacing of interest (up to $0.1 \mathrm{~m}$ ). Maximum potential difference varies much more rapidly with electrode spacing than does the maximum electric field. 
Kilpatrick's criterion also limits the amount of negative charge which can be placed on a target without vacuum sparking. The surface potential and electric fields of a charged sphere are given by

$$
\begin{gathered}
E=\frac{q}{4 \pi \varepsilon_{o} r^{2}} \text { and } \\
V=\frac{q}{4 \pi \varepsilon_{0} r}
\end{gathered}
$$

where $q$ is the charge in coulombs and $r$ is the radius in meters. The mathematical relationship between the surface potential and electric field is coincidentally the same in the case of a sphere as in the case of the steering electrodes. But the size range of interest is an order of magnitude smaller. Figure 5-3 shows the maximum surface potential and electric fields associated with a negatively charged spherical target up to $10 \mathrm{~mm}$ radius. 


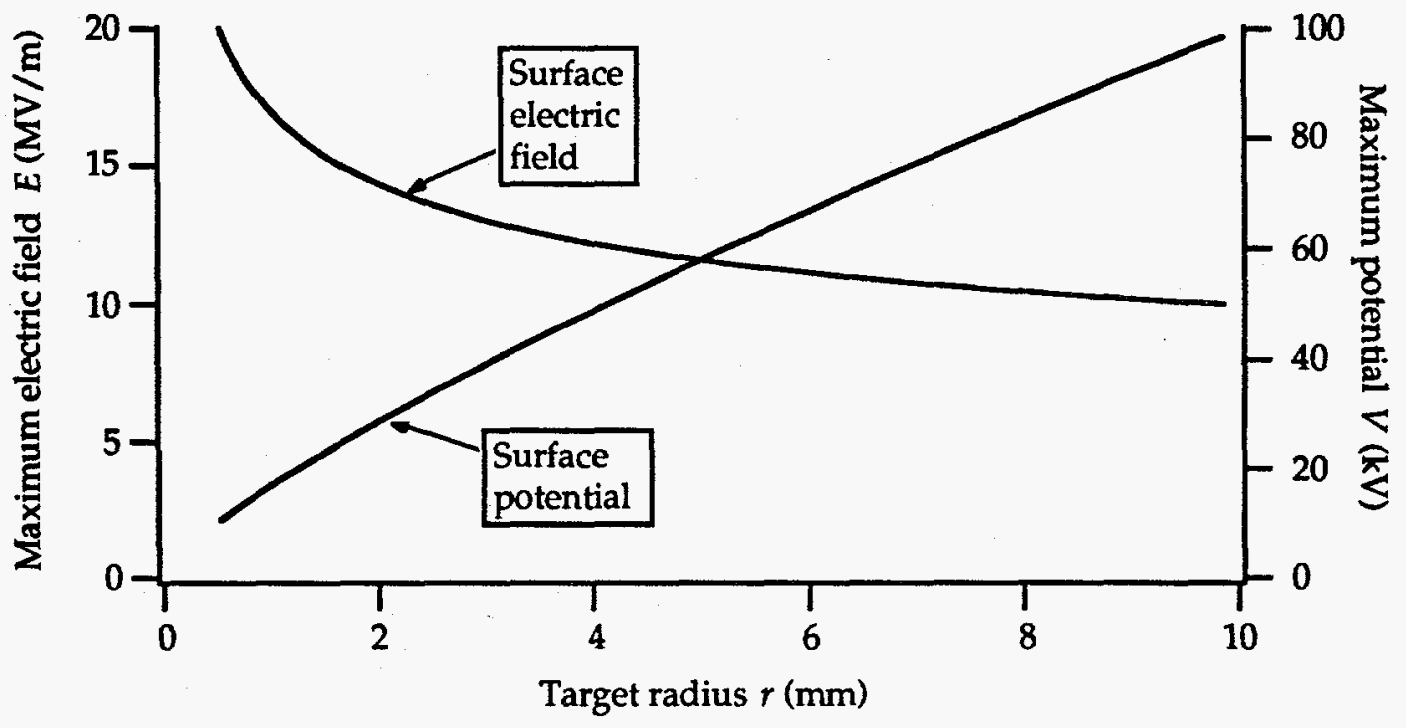

Figure 5-3. Maximum surface potential and electric field of a negatively charged sphere as functions of the sphere radius.

Figure 5-4 is a semilog plot of equations 5-2 to 5-4 for the negative charge holding ability of a spherical target as a function of target radius. Maximum charge held increases from 1.9 ncoulomb at $r=1 \mathrm{~mm}$ to 110 ncoulomb at $r=10$ $\mathrm{mm}$. If the target were positively charged, significantly larger (perhaps a factor of 10 larger) charge could be held without sparking. The reason for this is that a much larger local electric field is required to eject positive ions from a material (Field Ionization) than to eject electrons from a material (field emission). ${ }^{2}$ 


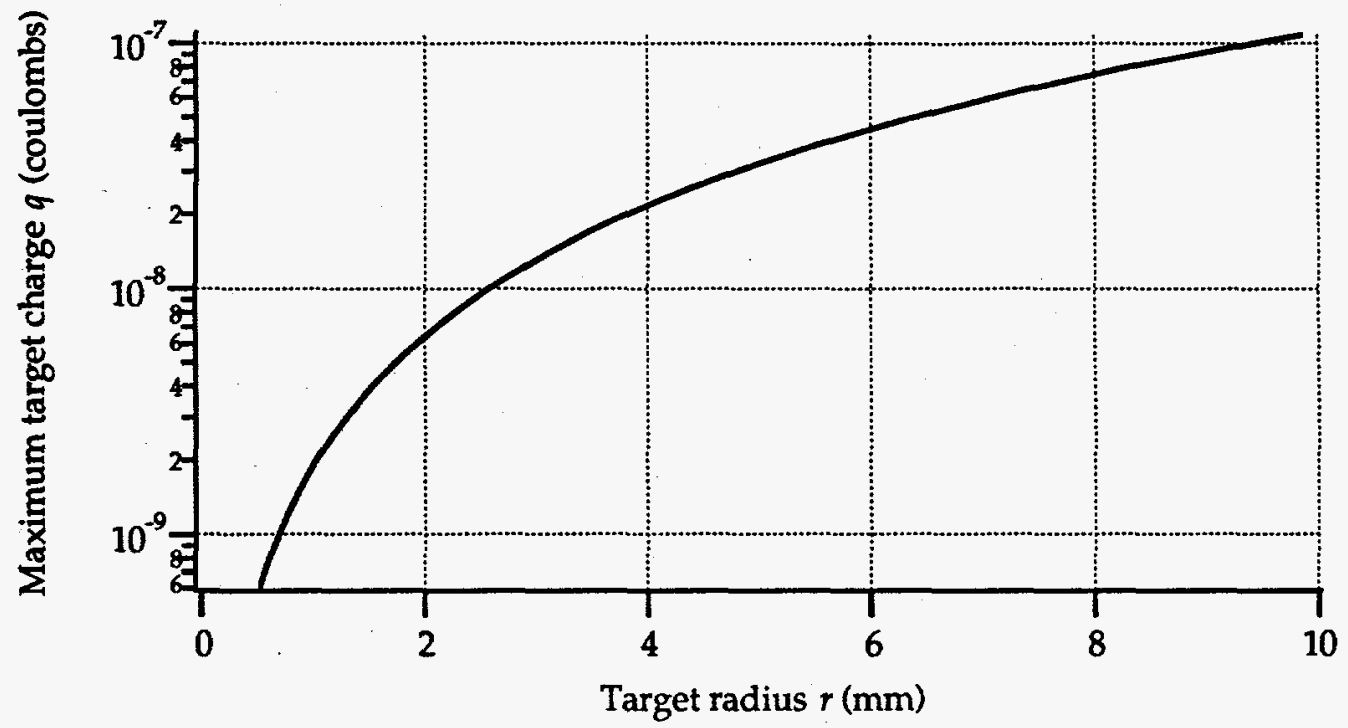

Figure 5-4. The amount of negative charge that a spherical target can hold without vacuum sparking increases rapidly with target radius. Substantially more positive charge could be held.

\section{Example 1.}

How far can a typical direct drive target be steered using this method?

Assume the following typical parameters:

Target radius

Target mass

Steering length

Free coasting length

Target speed

Electric field (Fig. 5-2)

Target charge (Fig. 5-4)

$$
r=3 \mathrm{~mm}
$$$$
m=0.03 \mathrm{~g}
$$$$
L_{s}=1 \mathrm{~m}
$$$$
L_{c}=5 \mathrm{~m}
$$$$
v=100 \mathrm{~m} / \mathrm{s}
$$

$$
E=6 \mathrm{MV} / \mathrm{m}
$$

$$
q=13 \text { ncoulomb }
$$

The displacement $\Delta Y$ is then given by equation (5-1). 


$$
\begin{aligned}
\Delta Y & =\frac{E q}{m v^{2}}\left\{\frac{L_{s}^{2}}{2}+L_{s} L_{c}\right\} \\
& =\frac{6 \times 10^{6} \mathrm{~V} / \mathrm{m}\left(1.3 \times 10^{-8} \mathrm{Coul}\right)}{3 \times 10^{-5} \mathrm{~kg}(100 \mathrm{~m} / \mathrm{s})^{2}}\left\{\frac{(1 \mathrm{~m})^{2}}{2}+1 \mathrm{~m}(5 \mathrm{~m})\right\} \\
& =1.4 \mathrm{~m} .
\end{aligned}
$$

This is three orders of magnitude more steering than would typically be required. The scaling of this steering can be seen from the above equation. The voltage can be decreased and the length $L_{s}$ can be shortened.

If only a small amount of steering is required ( $\Delta Y$ is small), the ratio of steering required and the required accuracy of the final target position is also small, so we need not know the values of the steering parameters precisely. On the other hand, if large steering is required, then the parameters must be well known. The fractional errors (e.g. $\Delta q / q$ or $2 \Delta v / v)$ in the parameters add in quadrature and the total should be less than $0.2 \mathrm{~mm} / \Delta Y$. The actual charge $q$ on the target may be the most difficult parameter to control precisely. It may be necessary to measure the charge on the target prior to steering and adjust the steering field accordingly.

\section{Example 2.}

Using the same parameters as in example 1, except a shorter steering length $\mathrm{L}_{\mathrm{s}}=0.5 \mathrm{~m}$, what steering electric field would be required to steer the target 10 $\mathrm{mm}$ ?

$$
E=\frac{\Delta Y m v^{2}}{q\left\{\frac{L_{s}^{2}}{2}+L_{s} L_{c}\right\}}=\frac{0.01 \mathrm{~m}\left(3 \times 10^{-5} \mathrm{~kg}\right)(100 \mathrm{~m} / \mathrm{s})^{2}}{1.3 \times 10^{-8} \operatorname{Coul}\left\{\frac{(0.5 \mathrm{~m})^{2}}{2}+(0.5 \mathrm{~m})(5 \mathrm{~m})\right\}}=88 \mathrm{kV} / \mathrm{m}
$$


If the steering electrodes were separated by $30 \mathrm{~mm}$, about $2.6 \mathrm{kV}$ potential difference would be required.

\subsection{Torque Effects}

As noted in chapter 2, indirect ion driven target tumble must be limited to about $1.5^{\circ}$

The torque that is applied to a target in an electric field has two main causes. The first cause is the charge separation on the target caused by the electric field of the electrodes. The second cause, which is covered later, is the charge attracted on the steering electrodes by the electric field of the target. As shown in Fig. 5-5, if a conducting target is not aligned perpendicular (or parallel) to the electric field, a charge separation will occur which will be acted on by forces that are not collinear. These forces provide a torque which tends to align the target axis parallel to the electric field. The equation for the magnitude of this torque $\tau$ is given by

$$
\tau=\int \mathrm{E} \times \mathbf{r} \rho d V=\int \mathrm{E} \times \mathbf{r} \sigma d A
$$

where $\mathbf{E}$ the electric field, $\mathrm{r}$ is the position vector measured from the targets center, $\rho$ is the volume charge density, and $\sigma$ is the surface charge density. 


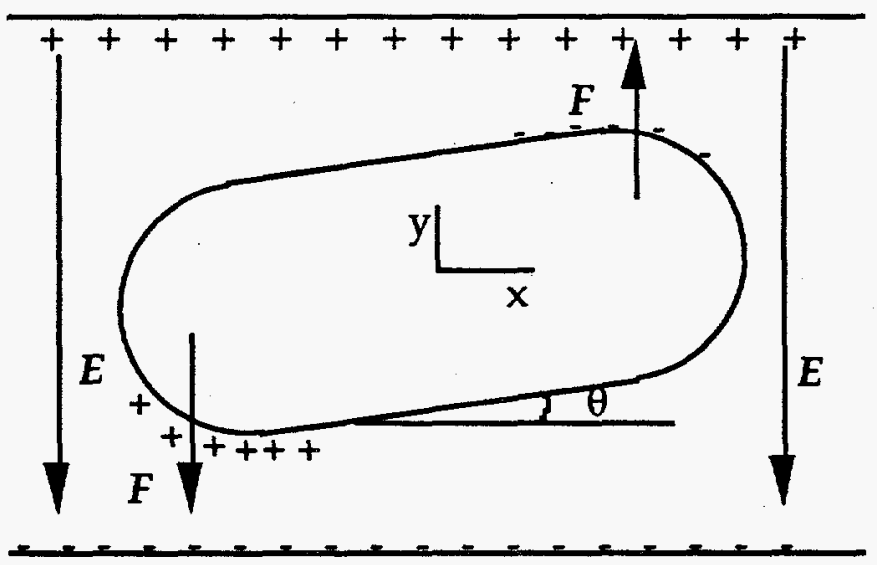

Figure 5-5. A conducting target in an electric field will undergo charge separation. A torque results which tends to align the target with the electric field.

Just as the field of the electrodes causes charge movement on the target, the field of the target causes charge movement on the electrodes which provides additional torque on the target.

\subsection{Computer Simulation}

Is the magnitude of the torque on a typical target sufficient to cause excessive target misalignment in the chamber center? This question is answered by using a three dimensional computer simulation. Due to electrical breakdown as discussed earlier, a target with sharp edges would not hold sufficient charge to allow adequate steering. Therefore, it is assumed that even indirect-drive targets requiring electrostatic steering would be made with rounded ends. The shape may be similar to a medicine capsule with straight sides and rounded ends.

As shown in Fig. 5-6, the model target has cylindrical sides of length $2 r_{0}$ with hemispherical ends of radius $r_{1}$. The target axis is assumed to be rotated an angle $\theta$ to the perpendicular to the applied field. 


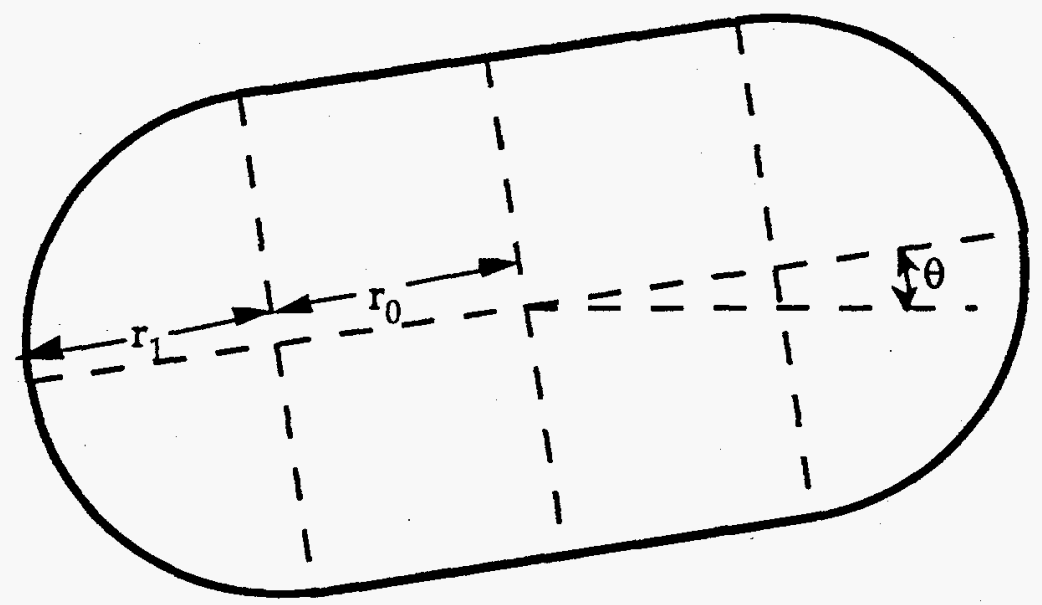

Figure 5-6. Dimensions of the model target for simulating the forces and torques on a target between steering electrodes.

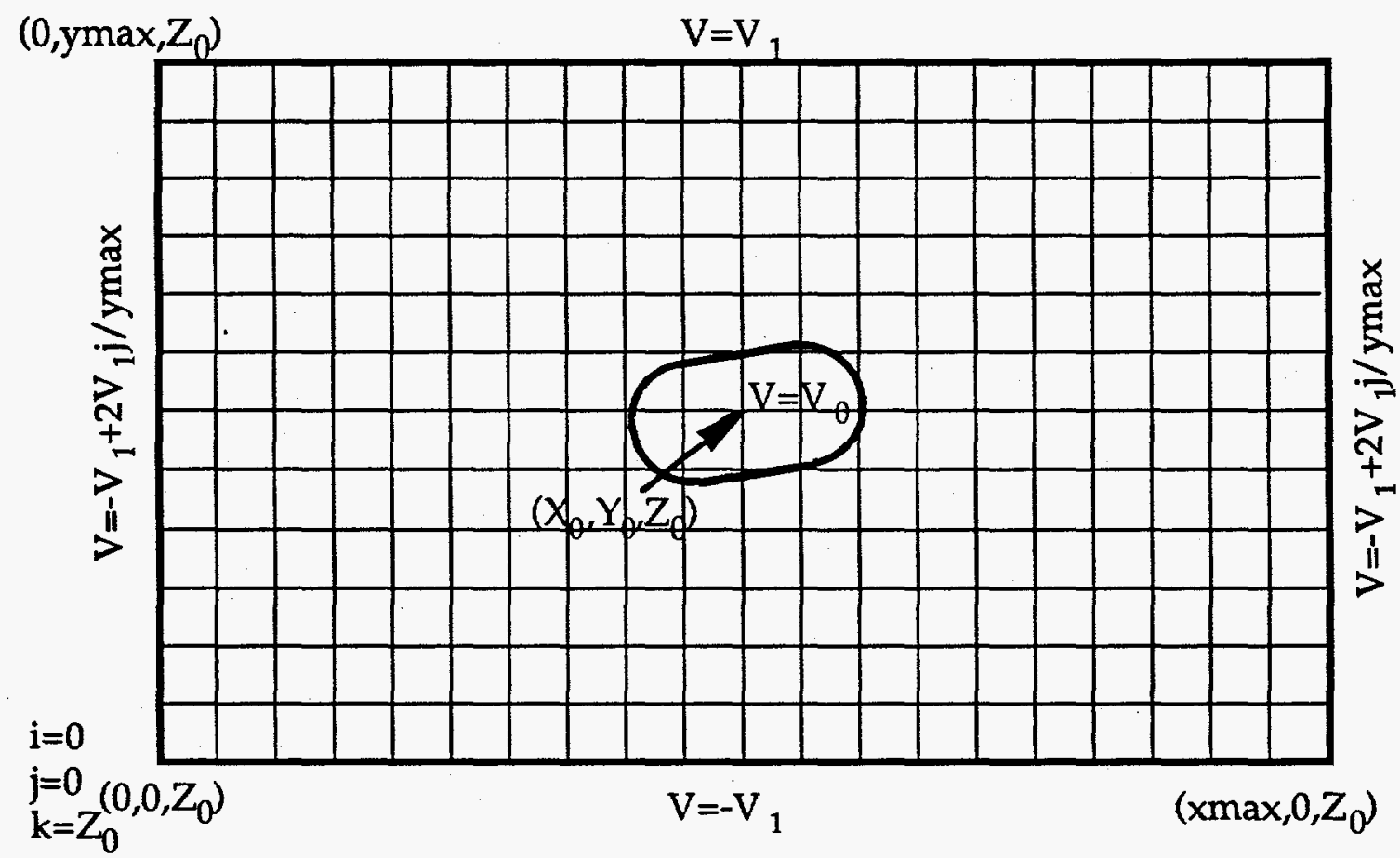

Figure 5-7. A three dimensional grid is set up to calculate the electric potential at each node of the grid.

As shown in Fig. 5-7, a grid is set up to compute the electric potential. Boundary potentials are assigned and held constant. The finite difference method with successive over relaxation is then used to solve Laplace's equation and 
compute the potential at each node on the grid. Using the computed potentials at the nodes, the average electric field values between the nodes can be calculated where desired.

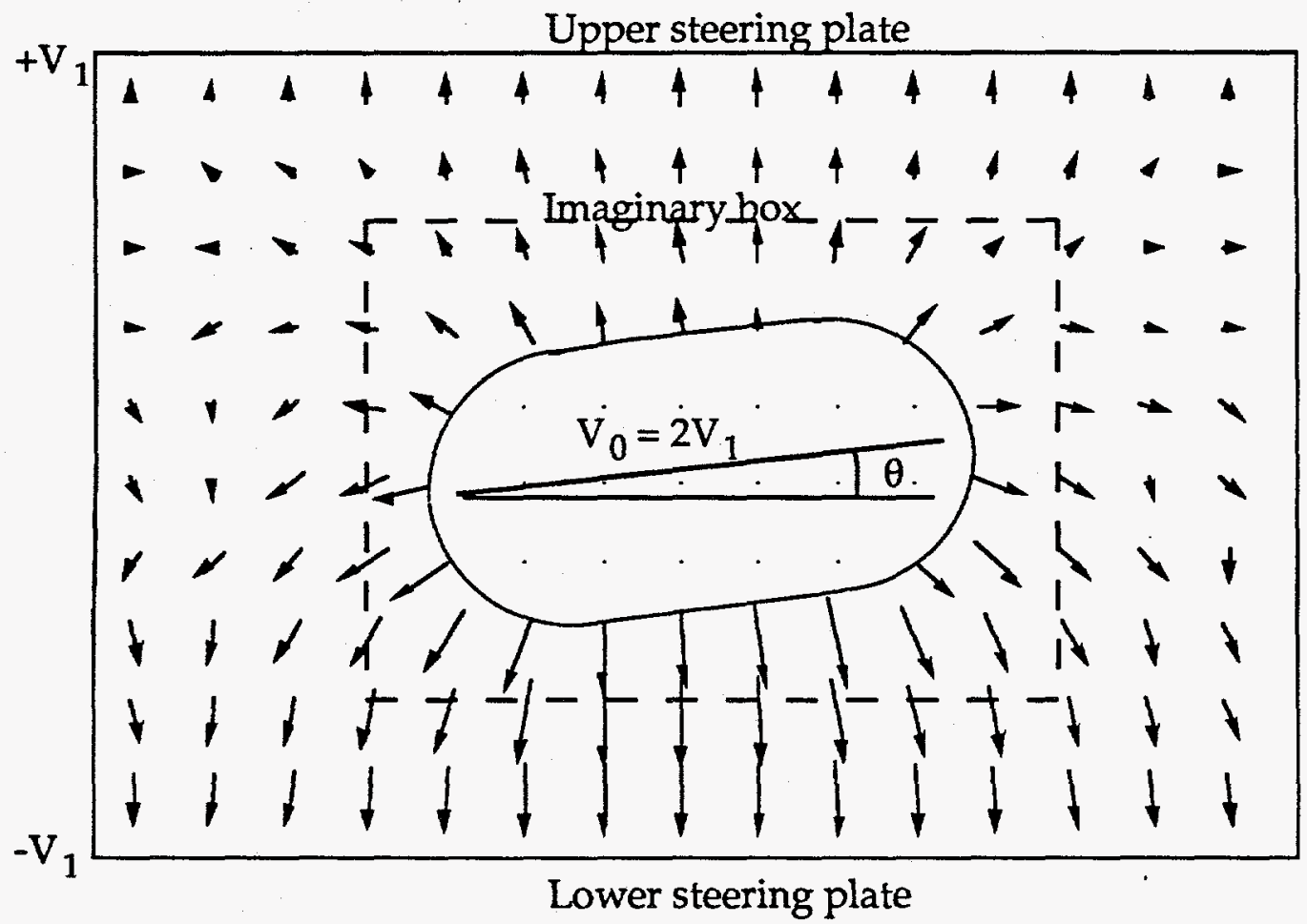

Figure 5-8. Electric field lines around target between steering electrodes. The imaginary box surrounds the target on all six sides.

Figure 5-8 provides a visual display of the electric field around a charged target between steering electrodes. Figure 5-8 was produced with the help of a commercial finite-element code ${ }^{3}$ assuming a two-dimensional target. Within an electric field, both pressure and tension exist $t^{4}$. There is a tension $T$ along the field lines and a pressure $\boldsymbol{P}$ perpendicular to the field lines.

$$
T=\varepsilon E^{2} / 2 \text { and } P=-\varepsilon E^{2} / 2
$$


where $\varepsilon$ is the material permittivity and the minus sign indicates repulsive force. The magnitude and direction of the force per unit area on a surface in an electric field depends on the location and orientation of the surface. If the surface is perpendicular to the field (as in the case of a conductor surface) the force per unit area is attractive, equals the tension, and is perpendicular to the surface. If the surface is parallel to the field, the force per unit area is repulsive, equals the pressure, and is again perpendicular to the surface. If the surface is oblique to the field, both the tension and the pressure act on the surface, in their usual directions, and the force is no longer perpendicular to the surface.

One could calculate the field on the surface of the target and integrate the tension components around the target to obtain the force and torque on the target. An easier way to obtain the same result is to set up an imaginary box around the target as indicated in Fig. 5-8. It can be shown using appropriate integrals of the pressure over the two surfaces, that the net force and torque acting external to the box equals the net force and torque on the target. We calculate the average electric fields along and perpendicular to line segments attaching adjacent nodes along the sides of the box. Using a method described in the following paragraphs, we then calculate and sum the force and torque contributions of the electric field on each area element defined by 4 adjacent nodes on the box. 


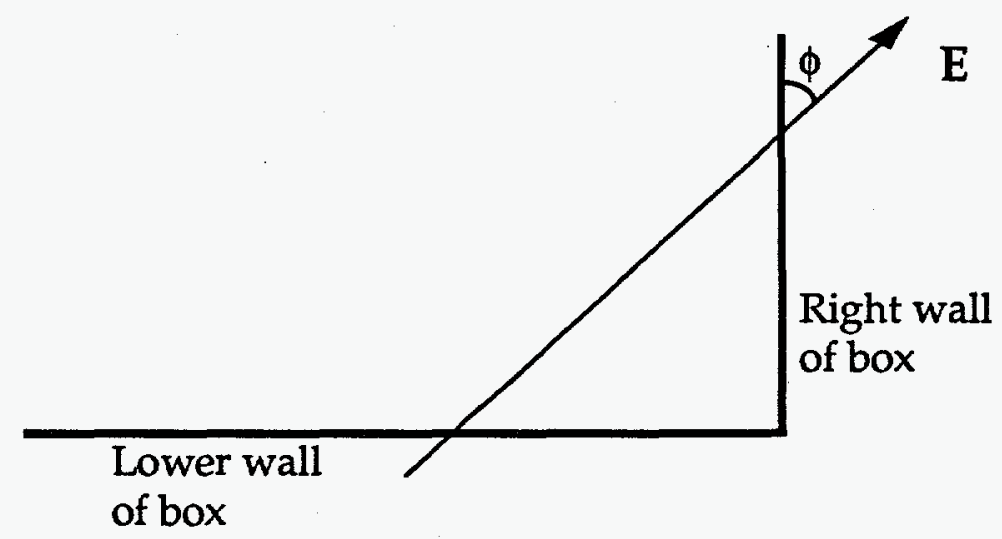

Figure 5-9. The lower right edge of the imaginary box surrounding the target. The force per unit area exerted on the walls of this box depend on the strength and direction of the electric field.

Once the electric field is known, the horizontal ( $x$ ) and vertical (y) components of the force per unit area can be calculated. Figure 5-9 assumes the field is in the $x-y$ plane. The generalization to three dimensions has been previously accomplished 5 . The $x$ component of the electric field is Esin $\phi$. The $y$ component of the electric field is Ecos $\phi$. On the right side, the tension along the field lines produces a force per unit area of $\varepsilon \mathrm{E}^{2} \sin \phi / 2$. The pressure perpendicular to the field lines exerts a force per unit area of $\varepsilon E^{2} \cos \phi / 2$. The net $x$ component of the force per unit area is then given by

$$
F_{x} / A=T_{x x}=\varepsilon\left(E^{2} \sin ^{2} \phi-E^{2} \cos ^{2} \phi\right) / 2=\varepsilon\left(\left(E_{x}\right)^{2}-\left(E_{y}\right)^{2}\right) / 2,
$$

where $\mathrm{T}$ is the electromagnetic field stress tensor known as Maxwell's stress tensor (the magnetic field terms are assumed to be negligible here).

Similarly,

$$
F_{y} / A=T_{x y}=\varepsilon\left(E^{2} \sin \phi \cos \phi+E^{2} \cos \phi \sin \phi\right) / 2=\varepsilon\left(2 E_{x} E_{y}\right) / 2=\varepsilon E_{x} E_{y} .
$$


With a $\mathrm{z}$ component to the field this result becomes

$$
\begin{gathered}
F_{x} / A=T_{x x}=\varepsilon\left(\left(E_{x}\right)^{2}-\left(E_{y}\right)^{2}-\left(E_{z}\right)^{2}\right) / 2 . \\
F_{y} / A=T_{y x}=\varepsilon E_{x} E_{y} \text { and } \\
F_{z} / A=T_{z x}=\varepsilon E_{x} E_{z} .
\end{gathered}
$$

The Maxwell stress tensor can be succinctly written ${ }^{5}$

$$
T_{\alpha \beta}=\varepsilon\left(E_{\alpha} E_{\beta}-1 / 2(E \cdot E) \delta_{\alpha \beta}\right)
$$

The force per unit area on a surface is the dot product of the stress tensor with the outwardly directed normal to the surface. Explicit results for all six sides assuming that $\mathrm{z}$ values increase from front to back are:

Left $\quad F_{x} / A=\varepsilon\left(-\left(E_{x}\right)^{2}+\left(E_{y}\right)^{2}+\left(E_{z}\right)^{2}\right) / 2, F_{y} / A=-\varepsilon E_{x} E_{y}$ and $F_{z} / A=-\varepsilon E_{x} E_{z}$

Right $\quad F_{x} / A=\varepsilon\left(\left(E_{x}\right)^{2}-\left(E_{y}\right)^{2}-\left(E_{z}\right)^{2}\right) / 2, F_{y} / A=\varepsilon E_{x} E_{y}$ and $F_{z} / A=\varepsilon E_{x} E_{z}$

Bottom $F_{y} / A=\varepsilon\left(\left(E_{x}\right)^{2}-\left(E_{y}\right)^{2}+\left(E_{z}\right)^{2}\right) / 2, F_{x} / A=-\varepsilon E_{x} E_{y}$, and $F_{z} / A=-\varepsilon E_{z} E_{y}$

Top $\quad F_{y} / A=\varepsilon\left(-\left(E_{x}\right)^{2}+\left(E_{y}\right)^{2}-\left(E_{z}\right)^{2}\right) / 2, F_{x} / A=\varepsilon E_{x} E_{y}$, and $F_{z} / A=\varepsilon E_{z} E_{y}$.

Back $\quad F_{z} / A=\varepsilon\left(\left(E_{x}\right)^{2}+\left(E_{y}\right)^{2}-\left(E_{z}\right)^{2}\right) / 2, F_{y} / A=-\varepsilon E_{z} E_{y}$, and $F_{x} / A=-\varepsilon E_{z} E_{x}$

Front $\quad F_{z} / A=\varepsilon\left(-\left(E_{x}\right)^{2}-\left(E_{y}\right)^{2}+\left(E_{z}\right)^{2}\right) / 2, F_{y} / A=\varepsilon E_{z} E_{y}$, and $F_{x} / A=\varepsilon E_{x} E_{z}$.

The surface area of each element is the grid spacing squared. The force per unit area between each node is then multiplied by the surface area to give the $x$, $y$, and $z$ components of the force on each element. The $x, y$, and $z$ forces are then summed all the way around the box. If the target is sufficiently far from the horizontal edges of the steering electrodes, the net horizontal force on the target approaches zero. The voltage may be applied after the target enters between the 
steering electrodes, and removed before the target leaves the steering electrodes to ensure the target is away from the fringing fields. This voltage is a steering voltage and must be changed for each target anyway.

Due to the geometry involved, the $\mathrm{x}$ and $\mathrm{y}$ components of the total torque will be zero. The $\mathrm{z}$ component of the torque due to the force on each element is calculated by multiplying the $y$ component of the force by the $x$ displacement of the element from the target center and subtracting the $x$ component of the force times the $y$ displacement of the element from the target center. If the center of the element is at point $(x, y, z)$ and the center of the target is located at point $\left(x_{0}, y_{0}, z_{0}\right)$, then the torque $T_{e l}$ due to that element is given by

$$
T_{e l}=F_{y}\left(x-x_{0}\right)-F_{x}\left(y-y_{0}\right)
$$

The torque is then summed around the imaginary box to yield the total torque on the target.

It is also possible to compute how much charge is on the model target. We use Gauss's law that the electric flux leaving the box is proportional to the charge enclosed. We simply multiply the normal component of the electric field passing through each element by the area of the element to obtain the flux leaving each element. We then sum these flux values multiplied by the permittivity to obtain the charge enclosed.

A series of computations was performed assuming the target was centered between electrodes spaced $16 \mathrm{~mm}$ apart with a rotation angle of 0.1 radians $\left(5.7^{\circ}\right)$. The target charge, target torque, target force, and maximum electric field at the cathode (for use in Kilpatrick's criterion) were calculated under various conditions. The conditions included cases with 1. target potential of $10,000 \mathrm{~V}$ with electrode potential $0 \mathrm{~V}$, 
2. target potential $0 \mathrm{~V}$ with electrode potential $\pm 10,000 \mathrm{~V}$, and

3. target potential $10,000 \mathrm{~V}$ with electrode potential $\pm 10,000 \mathrm{~V}$.

The computations were done with a three different target radii $r_{1}$ of $2.1 \mathrm{~mm}, 2.9$ $\mathrm{mm}$, and $4.4 \mathrm{~mm}$. Thirteen values were used for the cylinder half length's $\mathrm{r}_{0}$ ranging from $0.3 \mathrm{~mm}$ to $5.7 \mathrm{~mm}$. Additional computations were done to verify code accuracy and determine scaling laws which will be described later.

The results of these computations will now be provided in graphic form. Figures 5-10 and 5-11 show the maximum cathode field and the torque which result from an electrode potential of $\pm 10,000 \mathrm{~V}$ and a target potential of $0 \mathrm{~V}$. The target charge and net force on the target in this case are both zero.

Figures 5-12, 5-13, and 5-14 show the maximum cathode field, the torque, and the net charge which result from an electrode potential of $0 \mathrm{~V}$ and a target potential of $10,000 \mathrm{~V}$. The net force on the target is again zero in this case.

Figure 5-15 shows the net force that results from an electrode potential of $\pm 10,000 \mathrm{~V}$ and a target potential of $10,000 \mathrm{~V}$. The net torque, maximum cathode field and target charge can be obtained from the superposition principle by adding the separate results at $10,000 \mathrm{~V}$ target potential and $\pm 10,000 \mathrm{~V}$ electrode potential.

The following scaling laws apply. If the target length, radius, and electrode separation are all increased by the same factor " $x$ " while leaving the potentials the same, the charge and torque increase by $x$, the net force remains constant, and the electric fields change by $1 / x$. The target charge is directly proportional to the target potential. The net maximum cathode field is the sum of two terms. One $\left(E_{0}\right)$ is proportional to the target potential, the other $\left(E_{1}\right)$ is proportional to the electrode potential $\left(E_{\max }=E_{0}+E_{1}\right)$. The net force on the target is directly proportional to the product of the electrode and target potentials (equivalently the force is proportional to the product of the maximum cathode fields $\mathrm{F}=$ 
$\left.\mathrm{CE}_{0} \mathrm{E}_{1}\right)$. The torque on the target is the sum of two terms. One is proportional to the target potential (or max target induced cathode field) squared, the other is proportional to the electrode potential (or field) squared $\left(\mathrm{T}=\mathrm{A}\left(\mathrm{E}_{0}\right)^{2}+\mathrm{B}\left(\mathrm{E}_{1}\right)^{2}\right)$. For small target rotation angles, the net torque is proportional to the target rotation angle.

Kilpatrick's criterion, given by equation (5-2), limits the maximum cathode electric field $E_{\max }$ to about $10 \mathrm{MV} / \mathrm{m}$ for a potential difference of order 100,000 $V$. The positive potential electrode and the target are both surfaces to which sparking can occur. Therefore, the potential difference used in equation (5-2) is the greater of the potential difference between the electrodes and the potential difference between the cathode and target.

To minimize the amount of target rotation, it is desirable to maximize the ratio of steering force to torque on the target. We maximize

$$
\frac{F}{T}=\frac{C E_{0} E_{1}}{A E_{0}^{2}+B E_{1}^{2}}=\frac{C E_{0}\left(E_{\max }-E_{0}\right)}{A E_{0}^{2}+B\left(E_{\max }-E_{0}\right)^{2}} .
$$

Taking the derivative with respect to $E_{0}$ and setting it equal to zero gives

$$
E_{0}=E_{\max } \frac{B \pm \sqrt{B^{2}-(B-A) B}}{B-A} .
$$

The negative square root is used to give $\mathrm{E}_{0}$ between 0 and $\mathrm{E}_{\max }$. For the target and electrode sizes used in this report, all values for $E_{0}$ and $E_{1}$ were between $40 \%$ and $60 \%$ of $E_{\max }$. So assuming $E_{\max }$ is around $10 \mathrm{MV} / \mathrm{m}$ with $E_{0}$ and $E_{1}$ each 5 $\mathrm{MV} / \mathrm{m}$, we calculated torques and forces for target sizes and electrode spacing equal twice those used in the original computations. The results of these 
computations are shown in figures 5-16 and 5-17. These results are not quite optimized, but they are representative of what can be achieved.

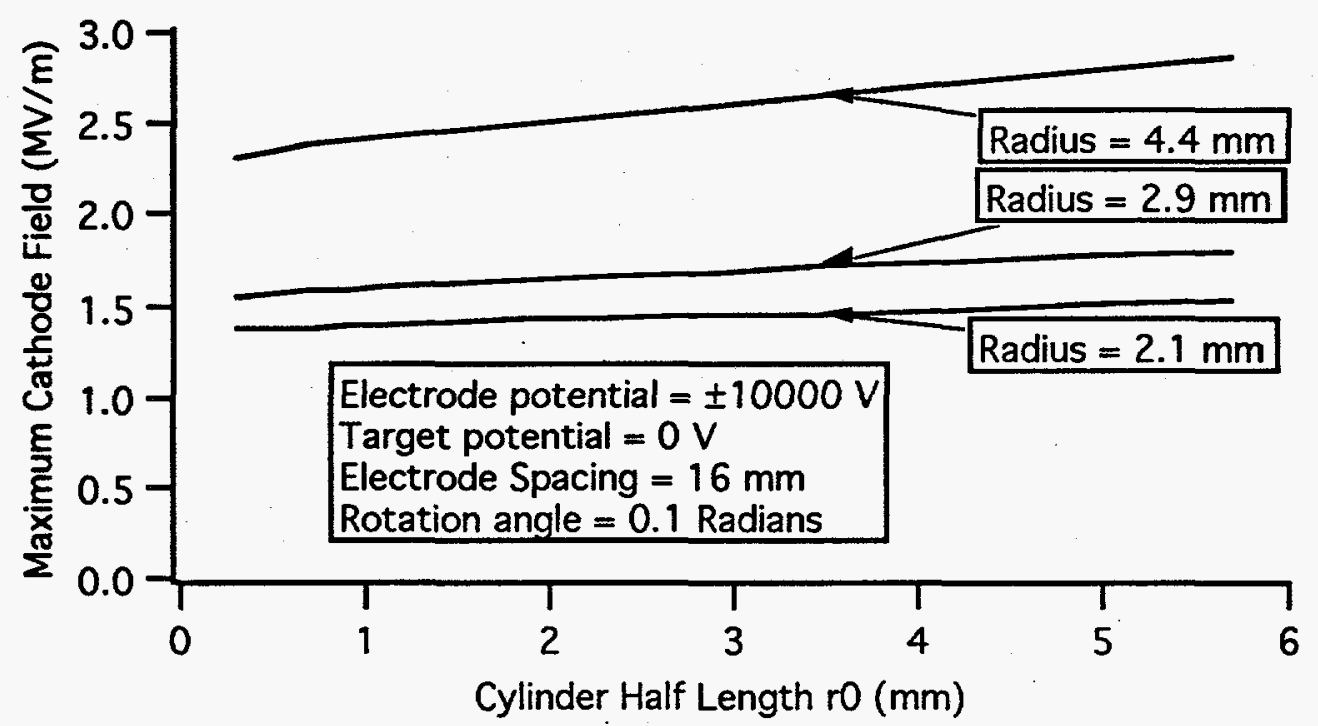

Figure 5-10. The maximum electric field at the cathode is plotted for a range of target lengths and three different target radii. Knowing the maximum cathode electric field is important to ensure vacuum sparking does not occur.

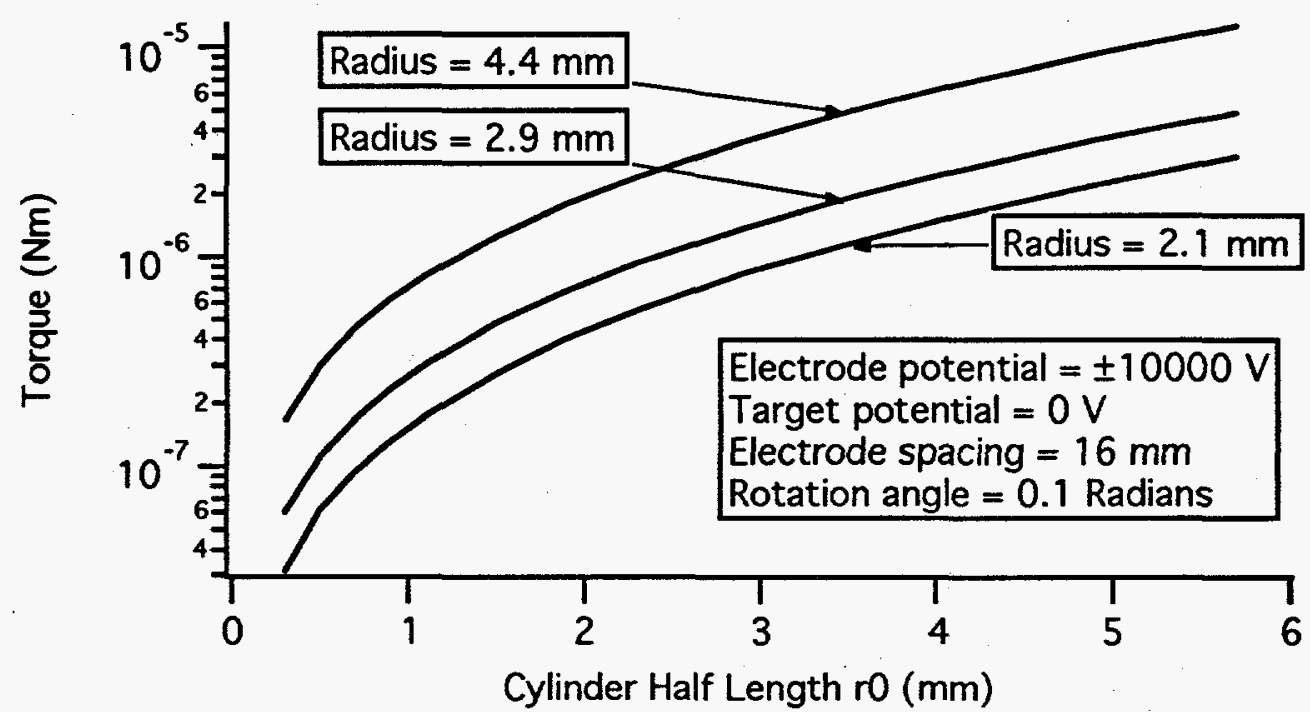

Figure 5-11. Torque on the target is plotted for a range of target lengths and three different target radii. 


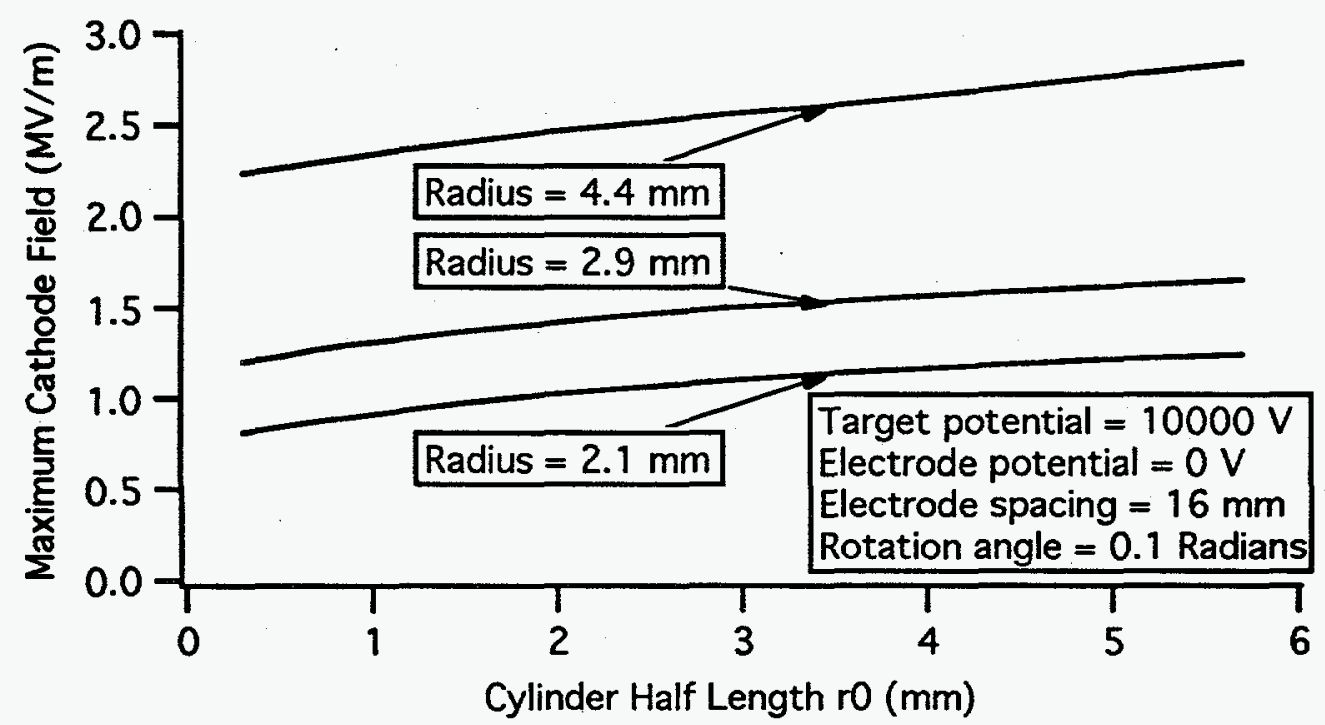

Figure 5-12. The maximum electric field at the cathode is plotted for a range of target lengths and three different target radii.

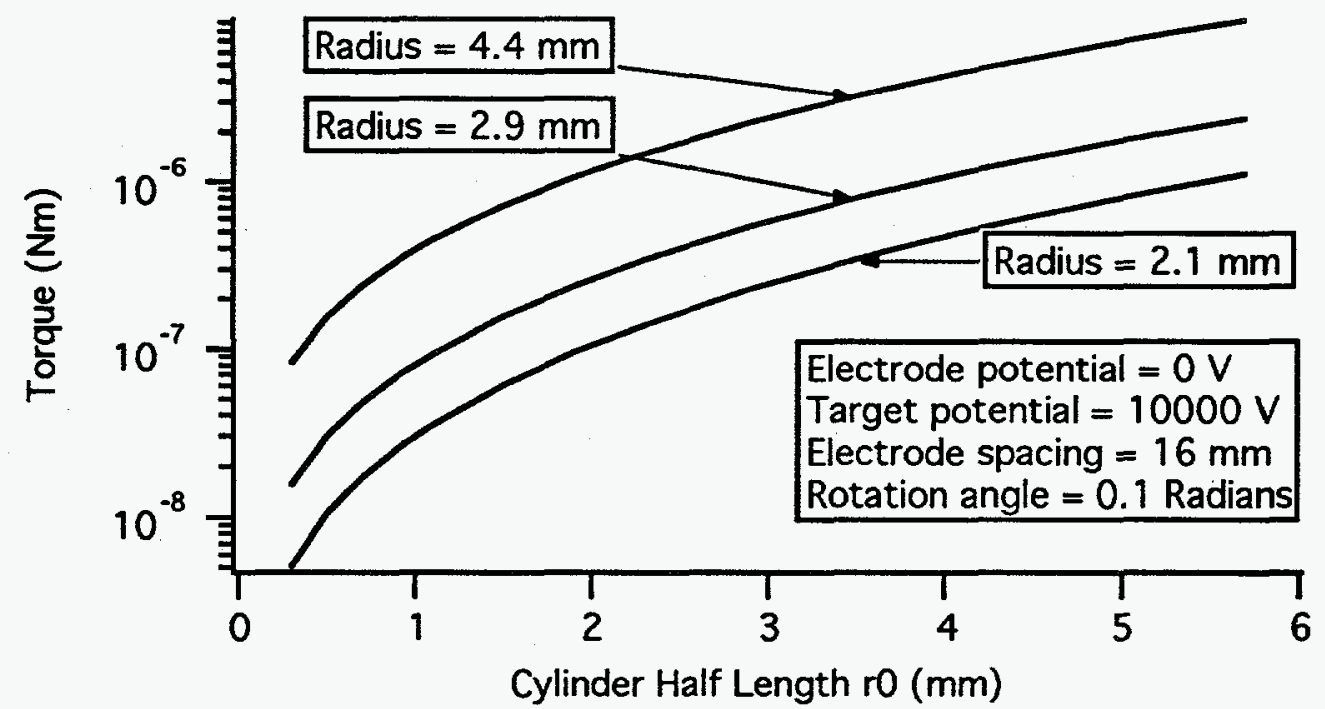

Figure 5-13. Torque on the target is plotted for a range of target lengths and three different target radii. 


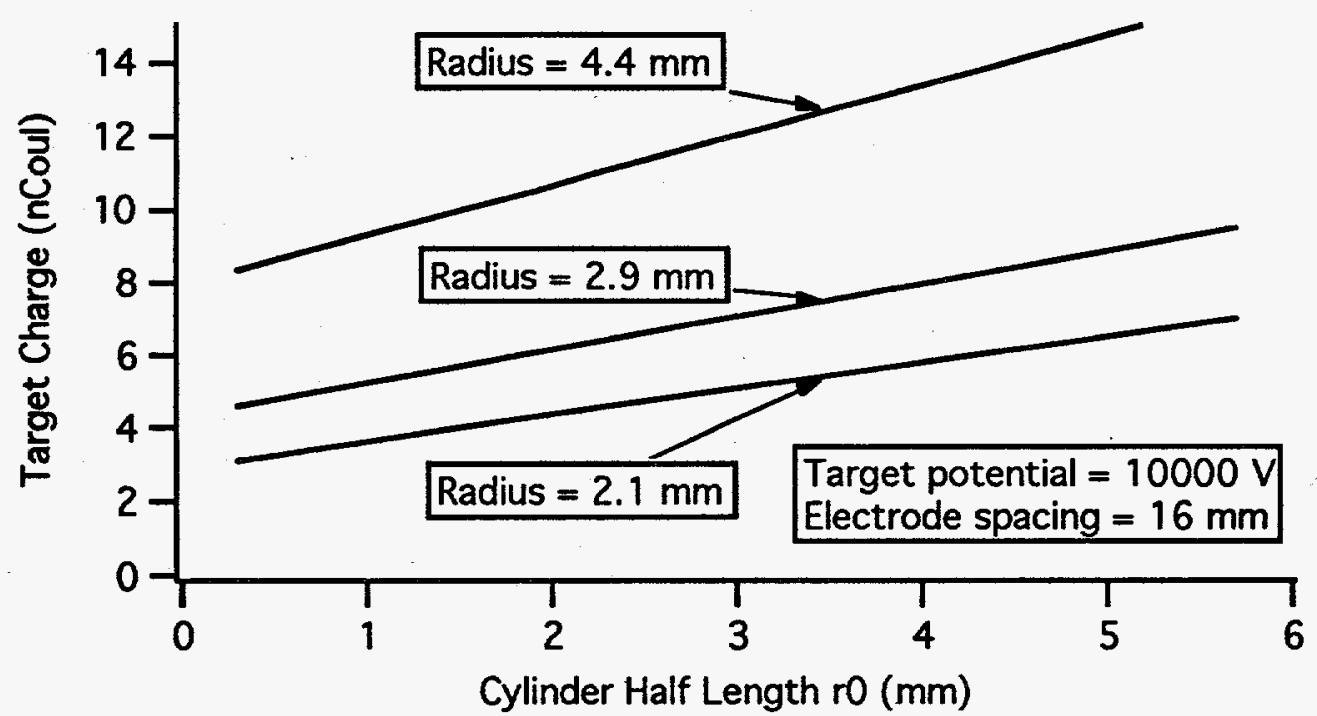

Figure 5-14. Charge on the target is plotted for a range of target lengths and three different target radii.

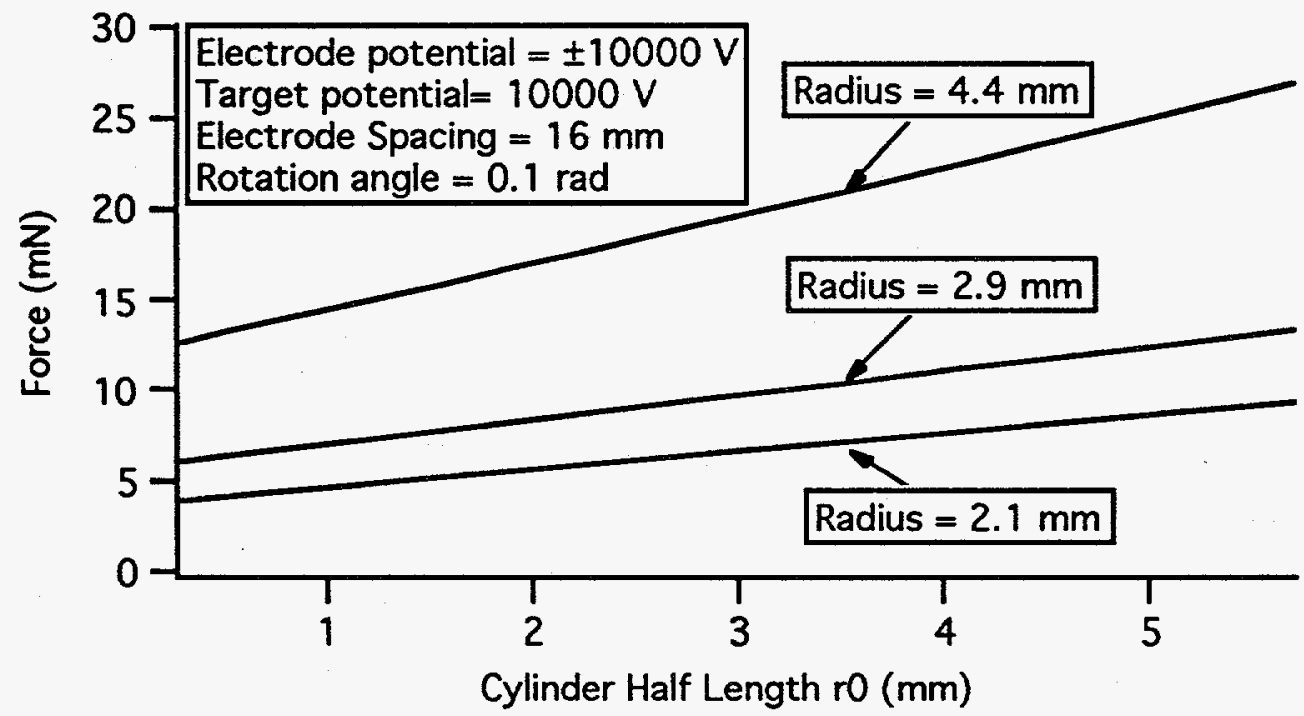

Figure 5-15. Net force on the target is plotted for a range of target lengths and three different target radii. 


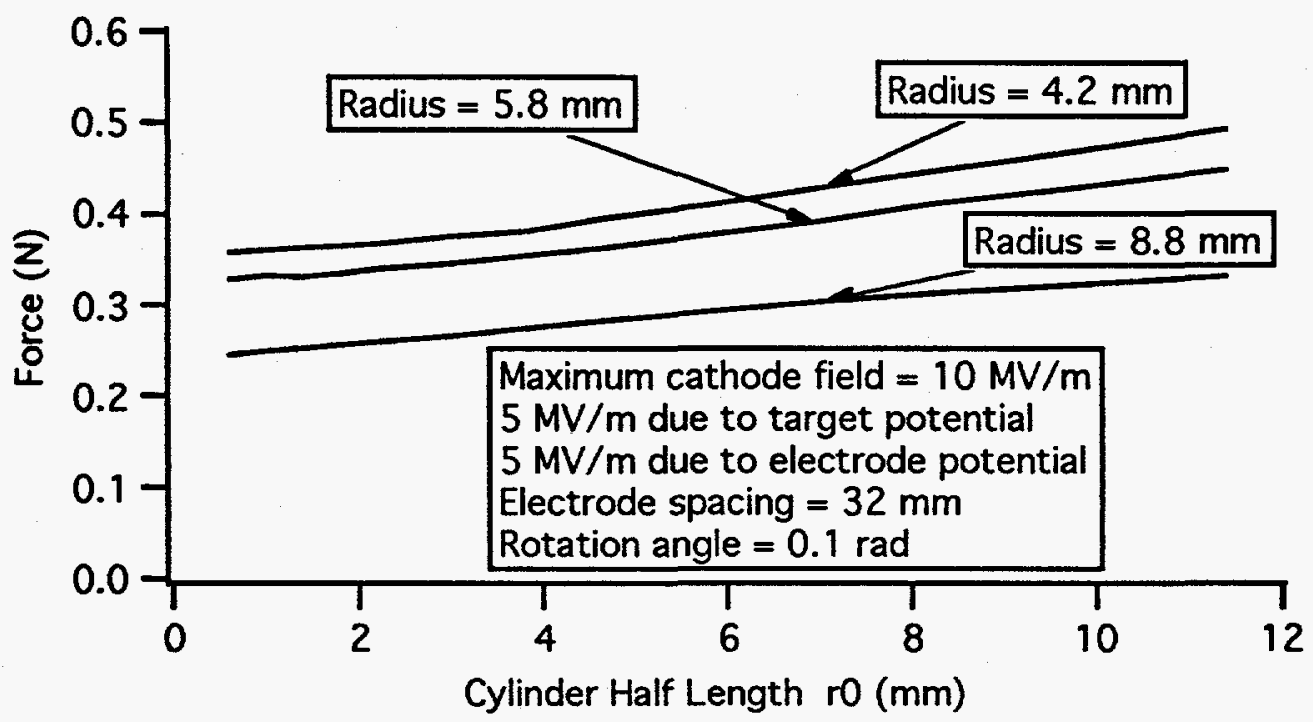

Figure 5-16. Net force on the target is plotted for a range of target lengths and three different target radii.

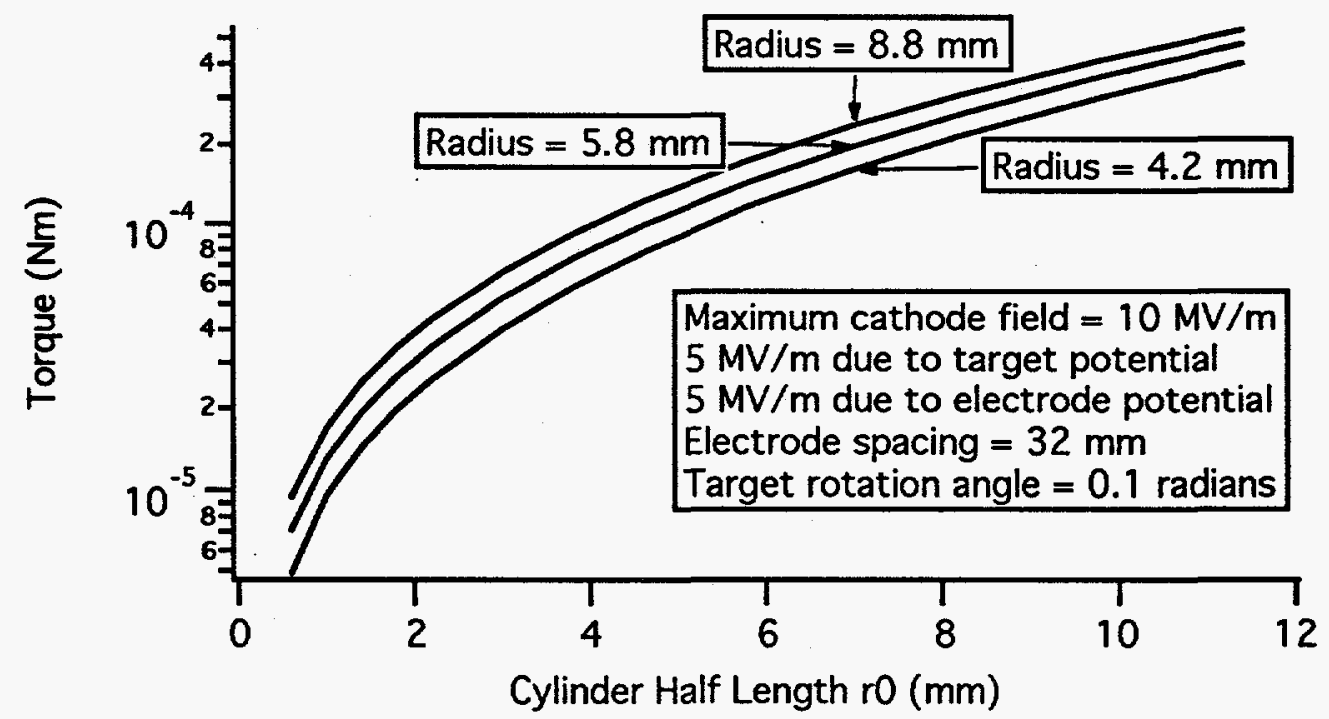

Figure 5-17. Torque on the target is plotted for a range of target lengths and three different target radii.

To calculate the rotation caused by the torque, the target moment of inertia must be known. Assuming the mass is uniformly distributed over the area of the target, the moment of inertia is given by 


$$
\begin{aligned}
& I=\frac{m}{6\left(r_{0}+r_{1}\right)}\left(4 r_{1}^{3}+3 r_{1}^{2} r_{0}+6 r_{1} r_{0}^{2}+2 r_{0}^{3}\right)=m r_{1}^{2} I^{\prime} \text { where } \\
& I^{\prime} \equiv \frac{1}{6\left(r_{0}^{\prime}+1\right)}\left(4+3 r_{0}^{\prime}+6 r_{0}^{\prime 2}+2 r_{0}^{\prime 3}\right) \text { and } r_{0}^{\prime} \equiv \frac{r_{0}}{r_{1}} .
\end{aligned}
$$

The normalized moment of inertia I' vs. the normalized target cylinder half length $r_{0} 0$ is plotted in Fig. 5-18. We see that I' varies from 0.67 to 2.8 over the range of interest of targets that are spherical to targets that are three times as long as they are wide. Of course one could calculate the actual moment of inertia of any specific target of interest.

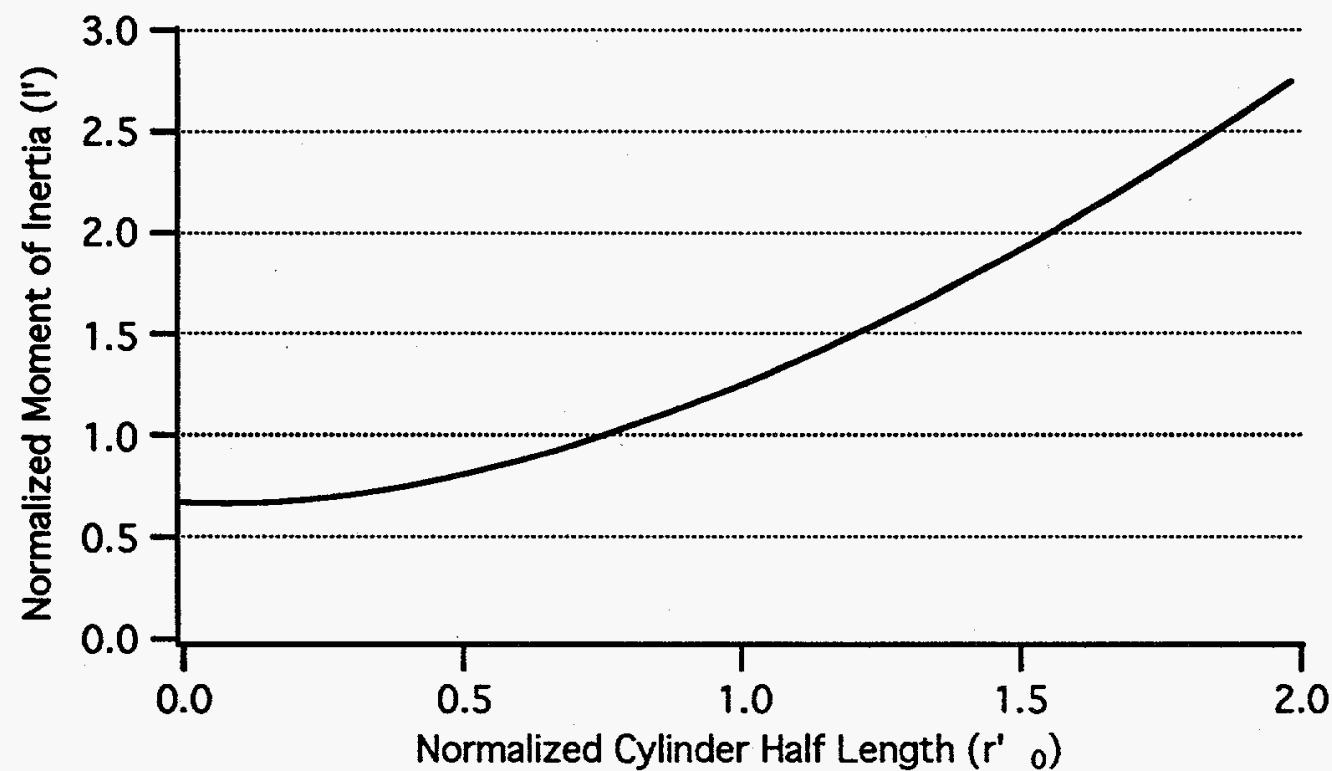

Figure 5-18. Normalized moment of inertia $\left(\mathrm{I}^{\prime}=\mathrm{I} / \mathrm{mr}_{1}{ }^{2}\right)$ vs. normalized target cylinder section half length $\left(\mathrm{r}_{0}=\mathrm{r}_{0} / \mathrm{r}_{1}\right)$.

We now have enough data to calculate how much steering can be achieved with a given amount of target rotation angle. If we assume that the steering electrodes are short compared to the coasting distance after passing through the electrodes (which will probably be at least $3 \mathrm{~m}$ ), then the target rotation angle will change relatively little while passing between the electrodes. In any case, the 
angle $\theta$ used in the computations should be the average target angle as it passes between the electrodes. The total angular change $\Delta \theta$ of the target due to steering is directly proportional to the torque $T$, which is proportional to $\theta$ for small angles.

$$
\Delta \theta=\frac{T}{I}\left(\frac{t_{s}^{2}}{2}+t_{s} t_{c}\right)=\frac{T t_{s}}{m r_{1}^{2} I^{\prime}}\left(\frac{t_{s}+2 t_{c}}{2}\right) \propto \theta
$$

where $t_{s}$ is the steering time and $t_{c}$ is the coasting time. The total linear change in the targets position due to steering is proportional to the force on the target.

$$
\Delta y=\frac{F}{m}\left(\frac{t_{s}^{2}}{2}+t_{s} t_{c}\right)=\frac{F t_{s}}{m}\left(\frac{t_{s}+2 t_{c}}{2}\right)
$$

Both, the linear and the angular change depend on the steering and coasting distance and target mass by the same relationships. Therefore, it is possible to calculate the amount of linear steering that will result in an additional target rotation angle that is equal to the initial target rotation angle, independent of the target mass, initial angle, steering distance, or coasting distance.

$$
\Delta y=r_{1}^{2} I^{\prime} \frac{F}{T} \text { for } \Delta \theta=\theta \text { and } T=T(\theta)
$$

Less linear steering would require less electric field and would result in proportionately less angular steering. 


\section{Example:}

A target has a radius of $5.8 \mathrm{~mm}$ and is twice as long as it is wide (hence $\mathrm{r}_{0}=$ $\left.\mathrm{r}_{1}\right)$. How much linear steering between electrodes placed $32 \mathrm{~mm}$ apart would result in approximately doubling the targets rotation angle? How much steering could be provided if the target mass was $1 \mathrm{~g}$, speed was $100 \mathrm{~m} / \mathrm{s}$, the steering distance was $1 \mathrm{~m}$ and the coasting distance was $5 \mathrm{~m}$ ?

\section{Answer:}

The maximum force, torque, and normalized moment of inertia are read from figures 5-16, 5-17, and 5-18 respectively. From equation (5-19),

$$
\Delta y=r_{1}^{2} I^{\prime} \frac{F}{T}=\left(5.8 \times 10^{-3}\right)^{2}(1.3) \frac{0.35 \mathrm{~N}}{1.5 \times 10^{-4} \mathrm{Nm}}=0.1 \mathrm{~m}
$$

From equation (5-18), the steering that would result from $1 \mathrm{~m}$ steering distance and $5 \mathrm{~m}$ coasting distance is

$$
\Delta y=\frac{F t_{s}}{m}\left(\frac{t_{s}+2 t_{c}}{2}\right)=\frac{0.35 \mathrm{~N}(0.01 \mathrm{~s})}{0.001 \mathrm{~kg}}\left(\frac{0.01 \mathrm{~s}+2(0.05 \mathrm{~s})}{2}\right)=0.2 \mathrm{~m}
$$

We are reminded here that greater steering is possible with larger steering and coasting distances at the expense of greater angular displacement. However, we do not expect to have to steer targets more than about $1 \mathrm{~mm}$, so these results are quite favorable. We would actually use a much lower electric field and a shorter steering distance appropriate to the amount of steering required. This would result in much less target rotation. 
This example assumed values for target size and electrode separation that required no use of scaling laws or interpolation. The following example demonstrates the use of these methods.

\section{Example:}

A target has a radius of $10 \mathrm{~mm}$ and cylinder half length of $5 \mathrm{~mm}$. How much linear steering between electrodes placed $40 \mathrm{~mm}$ apart would result in approximately doubling the targets rotation angle?

\section{Answer:}

We first scale the problem down by factor 2.5 to fit computed values in figures 5-10 through 5-15. $\mathrm{r}_{1}{ }^{\prime}=4 \mathrm{~mm}, \mathrm{r}_{0}{ }^{\prime}=2 \mathrm{~mm}$, and electrode spacing $=16 \mathrm{~mm}$. Then from Fig. 5-10; $\mathrm{E}_{1}{ }^{\prime}=2.2 \mathrm{MV} / \mathrm{m}$, from Fig. 5-11; $T_{1}{ }^{\prime}=1.5 \mu \mathrm{Nm}$, from Fig. 5-12; $\mathrm{E}_{0}{ }^{\prime}=2.2 \mathrm{MV} / \mathrm{m}$, from Fig. 5-13; $\mathrm{T}_{0}{ }^{\prime}=0.8 \mu \mathrm{Nm}$, and from Fig. 5-15; $\mathrm{F}^{\prime}=0.015 \mathrm{~N}$.

Scaling these values back up in size according to scaling rules gives $\mathrm{E}_{1}{ }^{\prime}=(2.2 \mathrm{MV} / \mathrm{m}) / 2.5=0.88 \mathrm{MV} / \mathrm{m}$, $\mathrm{T}_{1}{ }^{\prime \prime}=(1.5 \mu \mathrm{Nm}) \times 2.5=(3.75 \mu \mathrm{Nm})$ ， $\mathrm{E}_{0} "=(2.2 \mathrm{MV} / \mathrm{m}) / 2.5=0.84 \mathrm{MV} / \mathrm{m}$, $\mathrm{T}_{0}{ }^{\prime \prime}=(0.8 \mu \mathrm{Nm}) \times 2.5=2.0 \mu \mathrm{Nm}$, and $F^{\prime \prime}=(0.015 N)(1)$.

We now evaluate the constants $A$ and $B$ for use in equation 5-15.

$$
\begin{aligned}
& \mathrm{A}=\mathrm{T}_{0}{ }^{\prime \prime} /\left(\mathrm{E}_{0}{ }^{\prime \prime}\right)^{2}=2.0 \times 10^{-6} \mathrm{Nm} /\left(8.4 \times 10^{5} \mathrm{~V} / \mathrm{m}\right)^{2}=2.8 \times 10^{-18} \\
& B=\mathrm{T}_{1}{ }^{\prime \prime} /\left(\mathrm{E}_{1}{ }^{\prime \prime}\right)^{2}=3.75 \times 10^{-6} \mathrm{Nm} /\left(8.8 \times 10^{5} \mathrm{~V} / \mathrm{m}\right)^{2}=4.8 \times 10^{-18}
\end{aligned}
$$




$$
\begin{aligned}
E_{0}= & E_{\max } \frac{B-\sqrt{B^{2}-(B-A) B}}{B-A} \\
& =10 \mathrm{MV} / \mathrm{m} \frac{4.8-\sqrt{(4.8)^{2}-(4.8-2.8) 4.8}}{4.8-2.8}=5.7 \mathrm{MV} / \mathrm{m} \text { and } \\
E_{1}= & E_{\max }-E_{0}=4.3 \mathrm{MV} / \mathrm{m} . \\
F= & F^{\prime \prime}\left(\frac{E_{0} E_{1}}{E_{0}{ }^{\prime \prime} E_{1}^{\prime \prime}}\right)=0.015 \mathrm{~N} \frac{(4.3)(5.7)}{(0.88)(0.84)}=0.50 \mathrm{~N} \\
T_{0}= & T_{0}{ }^{\prime \prime}\left(\frac{E_{0}}{E_{0}{ }^{\prime \prime}}\right)^{2}=2 \mu \mathrm{Nm}\left(\frac{5.7}{0.84}\right)^{2}=92 \mu \mathrm{Nm}, \\
T_{1} & =T_{1}{ }^{\prime \prime}\left(\frac{E_{1}}{E_{1}{ }^{\prime \prime}}\right)^{2}=3.75 \mu \mathrm{Nm}\left(\frac{4.3}{0.88}\right)^{2}=90 \mu \mathrm{Nm}, \text { and } \\
T & =T_{0}+T_{1}=1.8 \times 10^{-4} \mathrm{Nm} .
\end{aligned}
$$

From Fig. 5-18, $\mathrm{I}^{\prime}=0.8$. And finally using equation (5-19),

$$
\Delta y=r_{1}^{2} I^{\prime} \frac{F}{T}=\left(10^{-2}\right)^{2}(0.8) \frac{0.5 \mathrm{~N}}{1.8 \times 10^{-4} \mathrm{Nm}}=0.2 \mathrm{~m} .
$$

In the previous two examples, the target was steered 100 to $200 \mathrm{~mm}$ to cause a doubling of the targets tumble angle. The increase in tumble angle is proportional to the amount the target is steered. Therefore, targets may be electrostatically steered many millimeters (i. e. $<10 \mathrm{~mm}$ ) without excessively increasing target tumble angle. 


\subsection{References}

1. W. D. Kilpatrick, "A Criterion for Vacuum Sparking Designed to Include Both rf and dc," Review of Scientific Instruments, 28(10), 824-826 (1957).

2. R. Gomer, Field Emission and Field Ionization (Harvard University Press, Cambridge, Massachusetts, 1961), p. 1.

3. S. A. Klein, W. A. Beckman, and G. E. Meyers, FEHT A Finite Element Analysis Program (F-Chart Software, Middleton, WI, 1991).

4. W. R. Smythe, Static and Dynamic Electricity, 3rd ed. (McGraw Hill, New York, 1968), pp. 14-17.

5. J. D. Jackson, Classical Electrodynamics, 2nd ed. (John Wiley \& Sons, New York, 1975), p. 239. 


\section{Chapter 6 - Target Injection Methods}

\subsection{Introduction}

As indicated in Fig. 6-1, the target injector is an important component of an inertial fusion energy power plant. The approximate performance parameters include the following. Target injection rates of several per second ( 6 for HYLIFEII). ${ }^{1}$ The injection speeds must be of order $100 \mathrm{~m} / \mathrm{s}$. The required target acceleration is nominally $2,000 \mathrm{~m} / \mathrm{s}^{2}$. Driver beams must be positioned to within $20 \%$ of beam spot size on heavy-ion targets, and $10 \%$ of target radius for direct laser driven targets. ${ }^{2}$ For a $2 \mathrm{~mm}$ radius ion beam, this gives allowed error of \pm 0.4 $\mathrm{mm}$. Assuming statistically equal error is due to beam pointing, target placement, and target tumble, each could contribute $\pm 0.23 \mathrm{~mm}$ to this error. This tumble error corresponds to about $\pm 1.5^{\circ}$. For a spherical direct-drive target, tumble is not a factor.

There are many methods of target injection that may be effective in delivering targets to meet the above parameters. The top injection candidates are the electrostatic accelerator, gas gun, rail gun, induction accelerator, and ferromagnetic accelerator. The type of injection mechanism chosen may be different for different power plant and target designs. The injection mechanism for a direct-drive target may well be different than the mechanism for an indirect-drive target. References 1, 2, and 3 all recommend gas gun target injection for targets with removable sabots or indirect-drive targets. Reference 4 compares the gas gun and ferromagnetic accelerator both using sabots. Reference 5 recommends gas gun for indirect-drive and the ferromagnetic accelerator with a sabot for direct drive. The ferromagnetic design uses a traveling magnetic field gradient to exert a force on a ferromagnetic cylinder which is placed inside the sabot. This injection method is described in Ref. 4. 


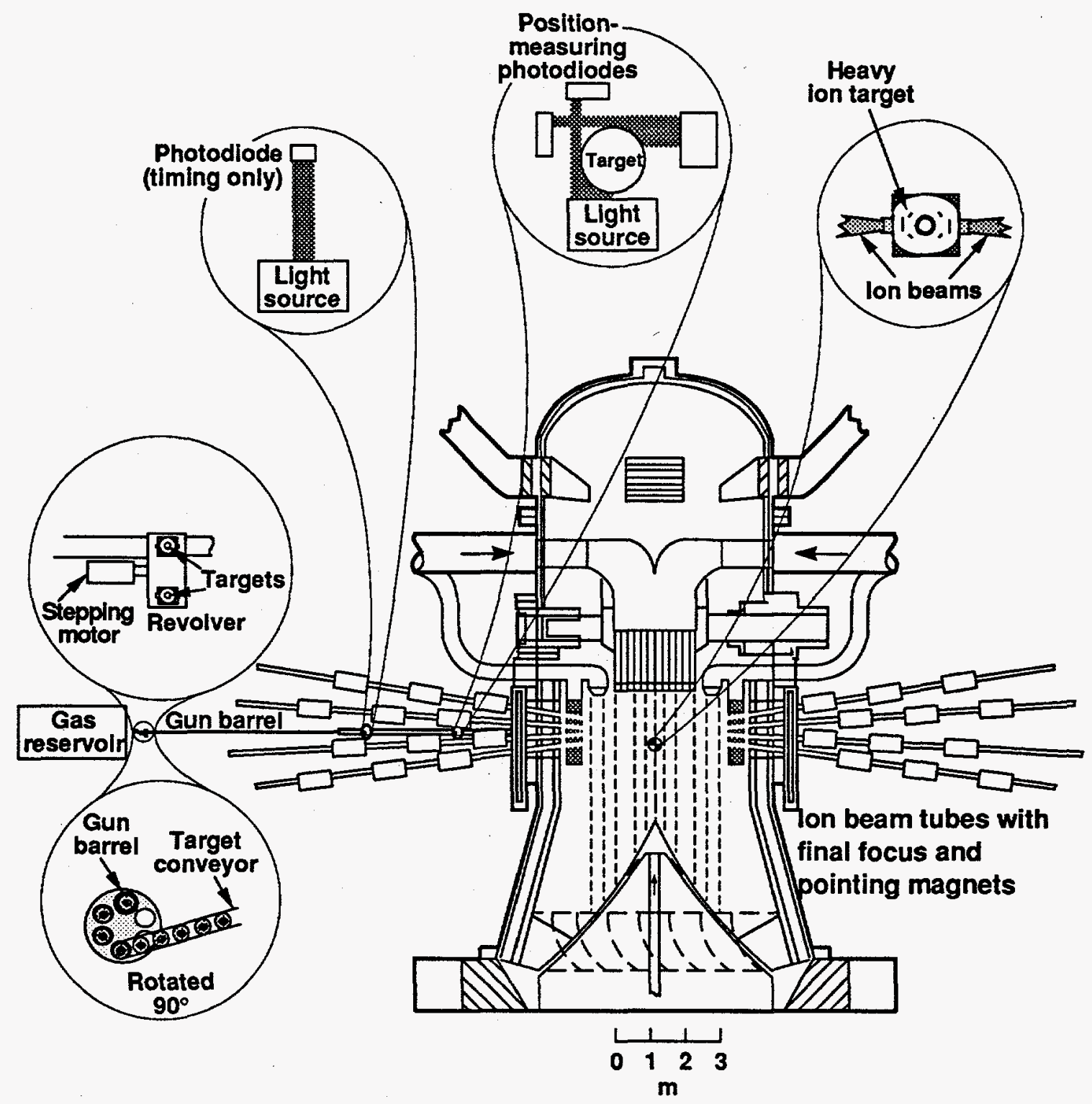

Figure 6-1. Inertial fusion energy reaction chamber with target injector and final part of driver beams.

The performance and cost of target injection systems are not well enough known to make quantitative target injector selection decisions. However, there are substantial qualitative differences that do indicate a preferred injector for various kinds of targets. The leading injection methods (except ferromagnetic 
acceleration) are discussed in the following sections. Table 6-1 summarizes the advantages and disadvantages of these methods.

Table 6-1. Advantages and disadvantages of leading injection methods.

\begin{tabular}{l|l|l}
\hline \hline $\begin{array}{l}\text { Injection } \\
\text { Method }\end{array}$ & Advantages & Disadvantages \\
\hline Gas gun & $\begin{array}{l}\text { - Simple } \\
\text { - Low risk of failure } \\
\text { - Low cost }\end{array}$ & $\begin{array}{l}\text { - Injects gas into vacuum chamber } \\
\text { requiring additional vacuum } \\
\text { pumping }\end{array}$ \\
\hline $\begin{array}{l}\text { Electrostatic } \\
\text { accelerator }\end{array}$ & $\begin{array}{l}\text { - Requires no sabot } \\
\text { injector }\end{array}$ & $\begin{array}{l}\text { - Limited propulsive force } \\
\text { - Difficult to control target position }\end{array}$ \\
\hline Rail gun & $\begin{array}{l}\text { Simple } \\
\text { Probably good speed } \\
\text { control }\end{array}$ & $\begin{array}{l}\text { - Requires electrical contact with rails } \\
\text { Possibly severe wear }\end{array}$ \\
\hline $\begin{array}{l}\text { Induction } \\
\text { accelerator }\end{array}$ & $\bullet$ No electrical contact & $\begin{array}{l}\text { be added to target } \\
\text { Requires many coils }\end{array}$ \\
\hline \hline
\end{tabular}

\subsection{Gas Gun}

\subsubsection{Gas Gun Overview}

A gas gun uses compressed gas (perhaps helium) to propel the target down a cylindrical barrel toward the reaction chamber. Some new concepts for a gas gun are indicated in Fig. 6-2. As described in section 6.2.2, the gas flow is controlled by a rotating cam and poppet valve arrangement allowing rapid (a few $\mathrm{ms}$ ) and reliable operation similar to an internal combustion engine. Reloading of the gun is accomplished via a revolver mechanism. A source of inaccuracy with a gas gun is gas flowing past the target after the target moves out of the barrel. This effect could be minimized by putting openings near the end of the gun barrel through 
which much of the gas can escape. Most of the gas escapes through the vents before it has a chance to disrupt the target trajectory and the gas may be differentially pumped prior to entering the reaction chamber.

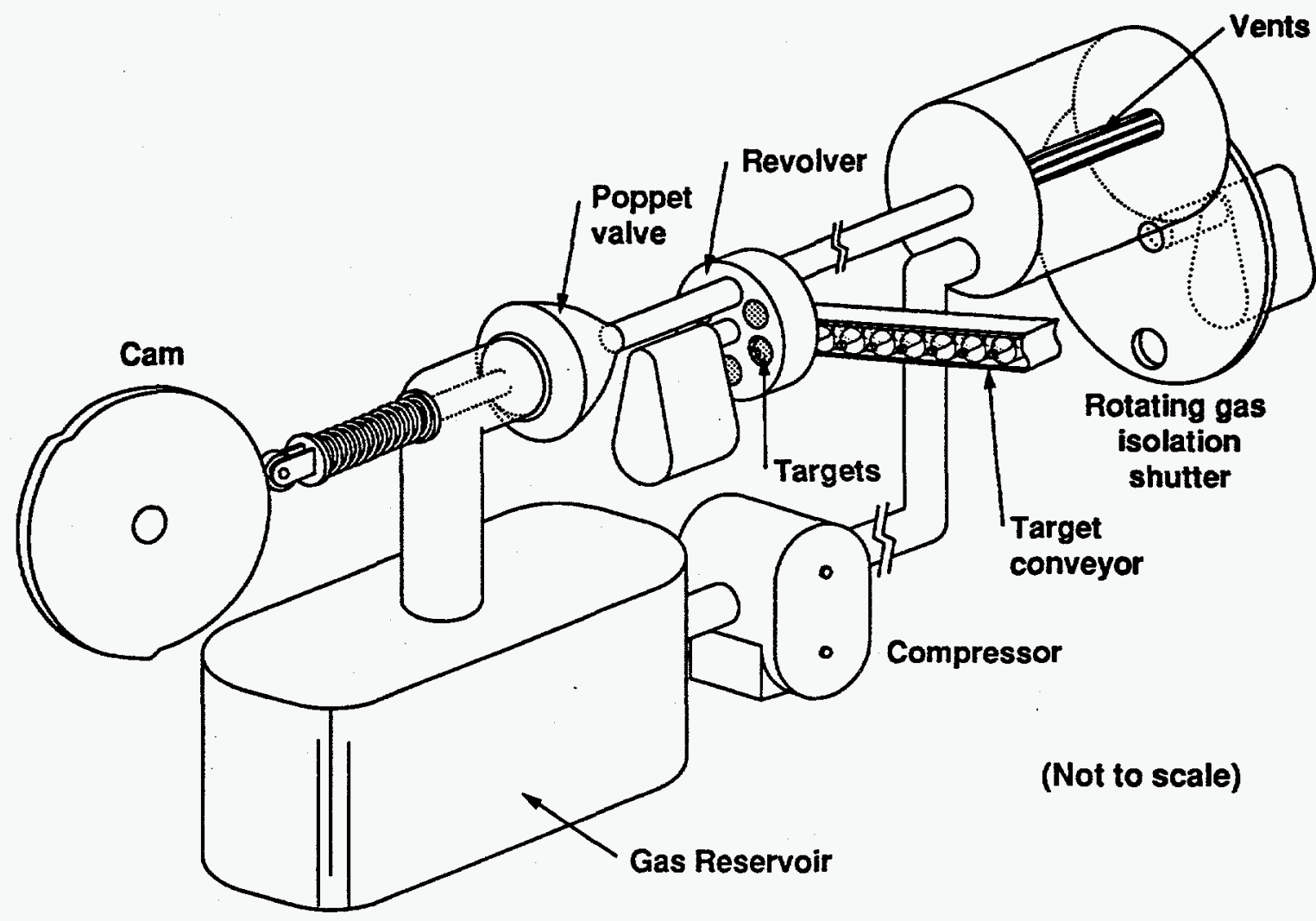

Figure 6-2. Gas gun target injector with vents for the gas to escape.

A target designed by Ho to which we have added a cylindrical target case and support membrane for injection purposes, is shown in Fig. 2-13.6 The mass of the basic target is about $0.5 \mathrm{~g}$, to this we add an additional $2 \mathrm{~g}$ of Flibe ( $\left.\mathrm{Li}_{2} \mathrm{BeF}_{4}\right){ }^{1}$ The Flibe is added to provide structure around a very thin layer of lead. Assume this $2.5 \mathrm{~g}, 7.5 \mathrm{~mm}$ radius target must be accelerated to a speed of $100 \mathrm{~m} / \mathrm{s}$ at a

\footnotetext{
${ }^{1}$ Flibe is derived from an acronym for fluorine, lithium, and beryllium. It is suggested for use here because in its molten form, Flibe is the first wall of the HYLIFE-II reaction chamber. ${ }^{1}$
} 
rate of $1,000 \mathrm{~m} / \mathrm{s}^{2}$ with room temperature helium. Simple calculations will verify that the gas pressure is $14 \mathrm{kPa}(2.1 \mathrm{psi})$ and about $20 \mathrm{mg}$ of gas is required. If the reference case of $2,000 \mathrm{~m} / \mathrm{s}^{2}$ acceleration is required, the gas pressure is doubled, but the mass remains the same. Removal of this gas and gas pressure drop in the barrel is covered in section 6.2.2.

To estimate the accuracy that can be expected, a comparison can be made with match quality air rifles. Air rifles are capable of firing test groups subtending $1 / 2 \mathrm{~min}(0.15 \mathrm{mrad})$ of angle at the standard $10 \mathrm{~m}$ range. ${ }^{7}$ This is a $\pm 0.75 \mathrm{~mm}$ spread at $10 \mathrm{~m}$. This accuracy should be met, or exceeded, firing through vacuum into a reaction chamber. If the accuracy can be improved (to $\pm 0.2 \mathrm{~mm}$ ) active target and beam steering would be unnecessary.

\subsubsection{Gas Flow Control and Pumping}

\subsubsection{Cam and Poppet Valve Flow Control}

When operating a gas gun for target injection purposes it is necessary to open and shut the gas control valve several times each second. The target acceleration time for a target speed of $100 \mathrm{~m} / \mathrm{s}$ would be between $10 \mathrm{~ms}$ (for acceleration of $10,000 \mathrm{~m} / \mathrm{s}^{2}$ ) and $100 \mathrm{~ms}$ (for acceleration of $1,000 \mathrm{~m} / \mathrm{s}^{2}$ ). Gas valve opening and shutting times should be a small fraction of the target acceleration time. To provide consistent target speeds which will improve injection accuracy, the opening and shutting times as well as the total time the valve is open should also be consistent.

Our method for achieving rapid, reliable, consistent gas control is borrowed from the internal combustion engine. In a typical four cycle engine, the valves open or shut in about $60^{\circ}$ of crankshaft revolution. 8 At $10,000 \mathrm{rpm}, 60^{\circ}$ of crank revolution requires just $1 \mathrm{~ms}$. 
An important design consideration for a gas gun is valve sizing. A typical poppet valve has a mean inlet flow coefficient of 0.35 with a lift to diameter ratio (L/D) of $0.25 .{ }^{9}$ While fully open ( $L / D>0.2$ ) a properly designed valve has a flow coefficient about $0.5 .^{9}$ This means that the open valve restricts flow similar to an orifice with half the area. If we design the valve with twice the area (1.4 times the diameter) of the gun barrel, it will not significantly reduce the flow when open. Sufficient flow area should also be provided to ensure the gas flow velocity remains below 0.6 times the speed of sound. Above 0.6 times sound speed valves become less efficient in transmitting gases. ${ }^{8}$ With the valve area twice the barrel area, the effective gas velocity throughout the valve is about the same as through the barrel. For example, a speed of $100 \mathrm{~m} / \mathrm{s}$ is about 0.1 times the speed of sound in room temperature helium. The diameter of typical automotive intake valves is over $4 \mathrm{~cm} .{ }^{8}$ The diameter of a typical IFE target is less than $2 \mathrm{~cm}$. The diameter of the gas gun control valve would be less than $3 \mathrm{~cm}$ diameter with about $0.75 \mathrm{~cm}$ lift.

Since the desired valve is smaller than an automotive valve, it could opened or shut more quickly than an automotive valve (i.e. $<1 \mathrm{~ms}$ ). However, the rotation speed of the cam to operate this gas valve will be much slower than the rotation speed of an automotive cam (e.g. $6 \mathrm{~Hz}$ for IFE vs. $83 \mathrm{~Hz}$ for a 10,000 RPM 4 cycle engine). With lower rotation speed, the cam must cause valve opening over a smaller rotation angle and a larger cam is required. If $3 \mathrm{~ms}$ corresponding to $6^{\circ}$ rotation is an acceptable opening time, then a cam with about a $15 \mathrm{~cm}$ radius could be used as illustrated in Fig. 6-3. 


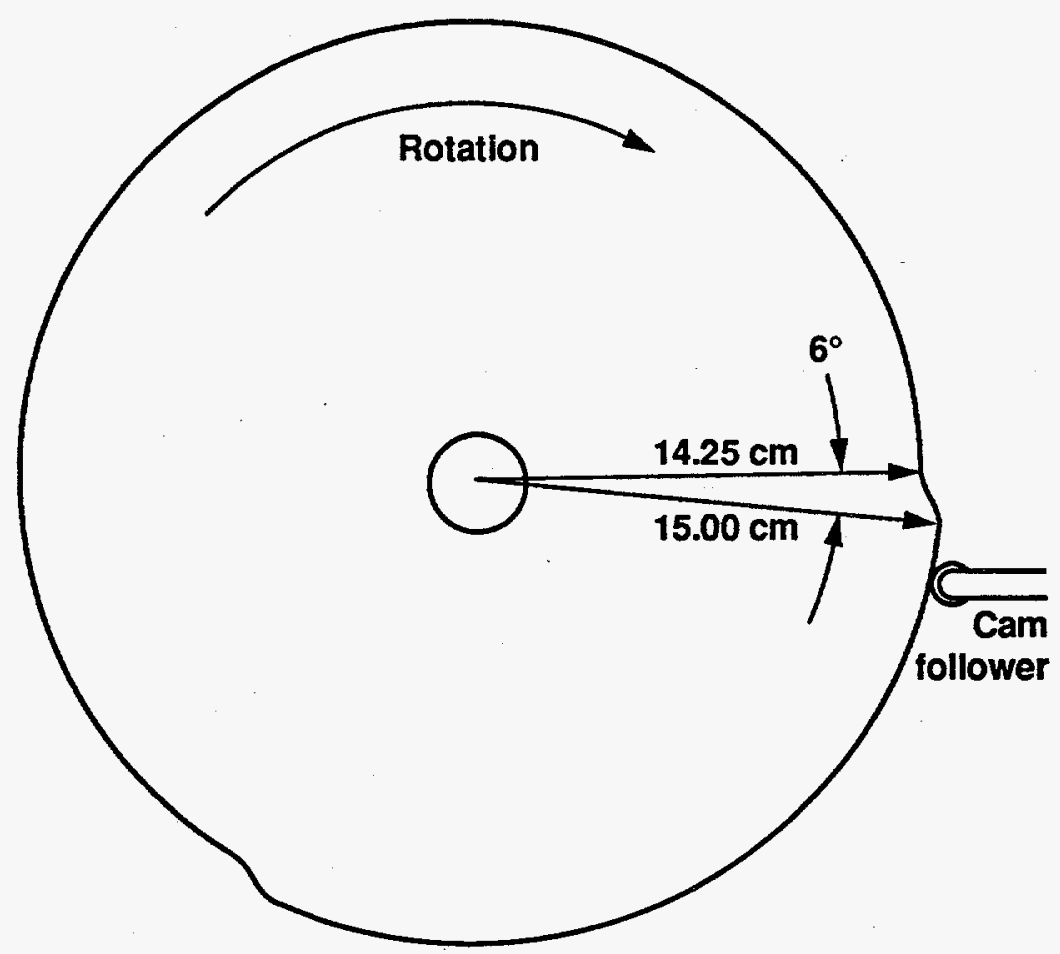

Figure 6-3. A cam for gas gun gas flow control.

\subsubsection{Vacuum Pumping Design}

We now consider removal of the helium gas used for injection. It should be noted that for a $400 \mathrm{MJ}$ fusion micro explosion, about $1 \mathrm{mg}$ of helium is produced and several milligrams of other gases such as deuterium and tritium are released. These gases must be removed from the reaction chamber and reprocessed. Therefore, it should not pose a problem if less than $1 \mathrm{mg}$ of helium from a gas gun enters the reaction chamber. Our gas removal design will be based on the previous example of $20 \mathrm{mg}$ per shot of room temperature helium flowing through a $5 \mathrm{~m}$ long, $7.5 \mathrm{~mm}$ radius gun barrel with a pressure of $14 \mathrm{kPa}$ and initial velocity of $100 \mathrm{~m} / \mathrm{s}$. A longer than reference case gun barrel length of $5 \mathrm{~m}$ is used here as. Shorter barrels will vent more quickly. The shutter and vents at the end of the gun barrel minimize the helium which proceeds past the end of the gun barrel. The helium expands out through the vents into a $0.09 \mathrm{~m}^{3}$ volume (100 times larger than the gun barrel volume) to allow the gas pressure to be reduced 
to $140 \mathrm{~Pa}$ ( 1 Torr). If a vacuum system is designed with capacity to pump $0.09 \mathrm{~m}^{3}$ each shot $\left(0.54 \mathrm{~m}^{3} / \mathrm{s}\right.$ or $1140 \mathrm{cfm}$ for a $6 \mathrm{~Hz}$ pulse rate), the average pressure can be maintained at about $140 \mathrm{~Pa}$. Commercial off the shelf Roots vacuum pumps with oil sealed mechanical backing pumps could handle this load for a purchase cost of about $\$ 40,000.10,11$ Acceptable pipe sizing leading to the Roots blower can readily be calculated. The mean free path $\lambda$ of the helium atoms is given by ${ }^{12}$

$$
\begin{aligned}
\lambda & =\frac{k T}{\pi P \delta^{2} \sqrt{2}} \\
& =\frac{1.38 \times 10^{-23} \mathrm{~J} / \mathrm{K}(293 \mathrm{~K})}{3.14(140 \mathrm{~Pa})\left(2.2 \times 10^{-10} \mathrm{~m}\right)^{2}(1.41)} \\
& =0.13 \mathrm{~mm}
\end{aligned}
$$

where $\delta$ is the gas kinetic diameter of the atoms. This is three orders of magnitude less than the expected piping size, so the flow is viscous rather than molecular. The gas flow $Q$ through the piping is equal to the product of the pressure $P$ and the volumetric flow rate $d V / d t$. $Q$ is also the product of the of the conductance $C$ and the pressure difference $\Delta P$. The viscous conductance of the piping is given by 12

$$
C=\frac{\pi}{128} \frac{D^{4}}{\eta L} \bar{P}
$$

where $\eta$ is the viscosity, $D$ is the pipe diameter, and $L$ is the pipe length. Assuming a $4 \mathrm{~m}$ pipe length (to allow the pump to be placed out of the congested beam area), we can calculate the minimum piping diameter to keep the pressure drop below $10 \%$ for example. 


$$
\begin{aligned}
D & =\sqrt[4]{\frac{128(d V / d t) \eta L}{\pi \Delta P}} \\
& =\sqrt[4]{\frac{128\left(0.54 \mathrm{~m}^{3} / \mathrm{s}\right)\left(1.96 \times 10^{-5} \mathrm{~kg} / \mathrm{s} \cdot \mathrm{m}\right)(4 \mathrm{~m})}{3.14(14 \mathrm{~Pa})}} \\
& =0.11 \mathrm{~m} .
\end{aligned}
$$

$0.11 \mathrm{~m}$ is smaller than the gas expansion volume diameter and is acceptable.

\subsubsection{Gun Barrel Venting}

It is of some interest to calculate how much gas pressure remains in the gun barrel after venting begins. The pressure gradient provides a force to propel the gas out of the gun barrel. This force is countered by a frictional force due to the gas viscosity. The magnitude of the frictional force is depends on whether the flow is laminar or turbulent. Reynolds number $N_{R e}$ for the example we have been considering is given by ${ }^{13}$

$$
\begin{aligned}
N_{R e} & =\frac{v D \rho}{\mu} \\
& =\frac{100 \mathrm{~m} / \mathrm{s}(0.015 \mathrm{~m})\left(\frac{2 \times 10^{-5} \mathrm{~kg}}{\pi(0.0075 \mathrm{~m})^{2}(5 \mathrm{~m})}\right)}{1.96 \times 10^{-5} \mathrm{~kg} / \mathrm{m} \cdot \mathrm{s}} \\
& =1700
\end{aligned}
$$

where $v$ is gas flow velocity. Since Reynolds number is less than 2,500 the flow is probably laminar. However, there will be some turbulence near the end of the gun barrel once venting begins because this gas will be accelerated to speeds much greater than $100 \mathrm{~m} / \mathrm{s}$. The resulting increased friction will reduce the initial venting rate. To calculate gas venting, we need equations for the friction in both 
the laminar and turbulent regimes. A friction coefficient $f$ can be defined as the ratio of the wall sheering stress $\tau_{\mathrm{w}}$ to the dynamic head of the gas ${ }^{13}$

$$
f \equiv \frac{\tau_{w}}{\rho v^{2} / 2}
$$

With this definition, for laminar flow the friction factor is given by 13

$$
f=\frac{16}{N_{R e}}=\frac{16 \mu}{\rho v D} .
$$

For turbulent flow and Reynolds numbers less than around 10,000 the friction factor is larger than is predicted for laminar flow and is roughly $0.01 .{ }^{13}$ There is a discontinuous increase in the friction factor going from laminar to turbulent flow. The cutoff between laminar and turbulent flow was assumed to occur at Reynolds number of 2500.

The frictional force $d F$ exerted by the gun wall on an element of length $d x$ of the gas is

$$
d F=\pi D \tau_{w} d x=\pi f D \rho v^{2} d x / 2
$$

For laminar flow, substituting equation (6-6) into equation (6-7) gives

$$
d F=8 \pi \mu v d x
$$

For laminar flow, the frictional force depends primarily on the gas velocity and is not affected by the gas pressure. As the pressure gradient decreases, the velocity of the gas will decrease causing the rate of pressure decrease to be greatly 
reduced. With turbulent flow, the friction force is pressure (density) dependent. The gas velocity is independent of the pressure gradient to density ratio. To calculate the gun barrel pressure as a function of time and position, I wrote a FORTRAN program in which the gas in the gun barrel is divided into 180 equal length elements. The assumed equal mass of these elements is calculated. These elements are allowed to expand and move due to the forces acting on them including the frictional force from the wall and the pressure forces from adjacent elements. The vents in the end of the gun barrel are assumed to be large enough so that gas flow is not restricted leaving the end of the barrel. The pressure volume relationship of the elements is assumed to be that of an isentropically expanding ideal gas and is given by

$$
P V^{\gamma}=P_{0} V_{0}^{\gamma}
$$

where $\gamma$ is the ratio of specific heat at constant pressure to specific heat at constant volume and is equal to 1.67. The gas in each element stays with the element. Once the center of the element passes out the end of the gun barrel its pressure is assumed to be zero. The position of the center of each element and the pressure of each element is computed vs. time for $100 \mathrm{~ms}$. The gas pressure vs. position is plotted at $10 \mathrm{~ms}$ intervals in Fig. 6-4. There will still be a few hundred $\mathrm{Pa}$ of pressure in the gun barrel when it comes time to accelerate the next target. If the external pressure is assumed to be $140 \mathrm{~Pa}$ instead of zero, the pressure approaches asymptotically to $140 \mathrm{~Pa}$ instead of to zero.

Also of interest is the pressure drop in the gun barrel during target acceleration which is given by 


$$
\Delta P=\frac{F}{A}=\frac{8 \pi \mu v L}{\pi r^{2}}=\frac{8\left(1.99 \times 10^{-5} \mathrm{~kg} / \mathrm{m} \cdot \mathrm{s}\right)(100 \mathrm{~m} / \mathrm{s})(5 \mathrm{~m})}{(0.0075 \mathrm{~m})^{2}}=280 \mathrm{~Pa}
$$

which is just $2 \%$ of the $14 \mathrm{kPa}$ accelerating gas pressure.

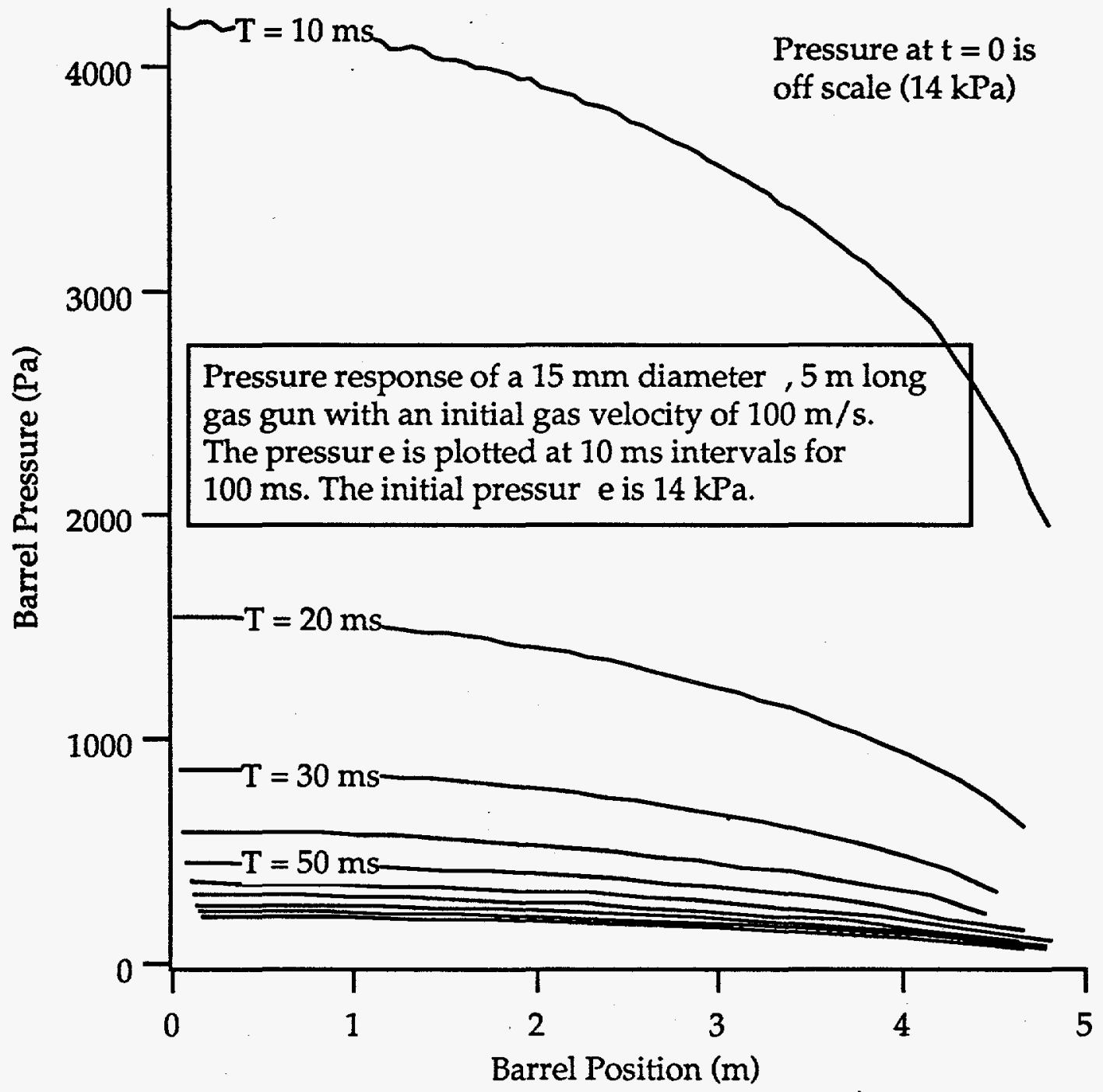

Figure 6-4. Barrel gas pressure vs. position for the first $100 \mathrm{~ms}$ after venting begins. Initial pressure is $14 \mathrm{kPa}$ of helium. The pressure is plotted at $10 \mathrm{~ms}$ intervals.

We now consider the pressure downstream of the gas gun through which the target must travel enroute to the reaction chamber. It is in this region that target 
position measurements are made and that steering takes place if necessary. The vents will be made large enough (i. e. with area much larger than the crosssectional area of the gun barrel) that the gas vents prior to reaching the end of the barrel. To find an upper limit to the quantity of gas that could enter this area, assume the gas in the expansion area around the vents proceeds with the speed of sound through the gun barrel end as it would through an open orifice into a vacuum. In this case the mass flow rate of gas down stream of the gun barrel would be given by

$$
\begin{aligned}
\frac{d m}{d t} & =\frac{\rho d V}{d t}=\frac{P W A c}{R T}=\frac{P W \pi r^{2} \sqrt{\not R T / W}}{R T}=P \pi r^{2} \sqrt{\frac{\gamma W}{R T}} \\
& =140 \mathrm{~Pa}(3.14)(0.0075 \mathrm{~m})^{2} \sqrt{\frac{1.67(0.004 \mathrm{~kg} / \mathrm{mole})}{8.3 \mathrm{~J} / \mathrm{mole} \cdot \mathrm{K}(300 \mathrm{~K})}}=40 \mathrm{mg} / \mathrm{s}
\end{aligned}
$$

where $W$ is the mass of a mole of helium, $A$ is the area of the gun barrel, and $c$ is the speed of sound. $40 \mathrm{mg} / \mathrm{s}$ is a third of the total $120 \mathrm{mg} / \mathrm{s}$ assumed to be used for a $6 \mathrm{~Hz}$ pulse rate. This gas flow can be greatly reduced by the use of a shutter at the end of the gun barrel. For example, it could be reduced by an order of magnitude if the shutter were shut $90 \%$ of the time. A rotating shutter as depicted in Fig. 6-2 could accomplish this task. In this case only about $3 \%$ of the total gas used makes it through the end of the gun barrel. If the expansion volume and pumping system were the same downstream of the barrel as they were around the vents, the pressure would be 30 times less or about $5 \mathrm{~Pa}$. With this low pressure, and a shutter isolating the reaction chamber, the gas entering the reaction chamber will be negligible.

An additional shutter and pumping stage could be provided to reduce

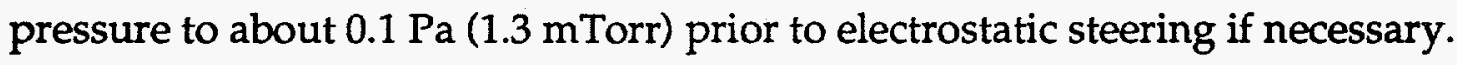
Some multistage Roots blowers have high pumping capacity at this low 
pressure. ${ }^{10}$ The ionization cross section for a single electron on a neutral helium peaks at 0.4 square angstroms. ${ }^{14}$ Therefore, at $0.1 \mathrm{~Pa}$, the mean free path of an electron for ionization of $\mathrm{He}$ is about $10 \mathrm{~m}$ which is much greater than the expected distance between steering electrodes. Therefore the gaseous helium should not increase sparking. However, the steering surfaces and target would have a monolayer of helium adhered. Good outgassing may not be possible.

Because of aerodynamic drag, excessive gas between the first target position detector and the reaction chamber could also affect target position prediction accuracy. However, $5 \mathrm{~Pa}$ is not nearly enough pressure to substantially degrade target accuracy.

The gas gun target injection method is recommended for indirect-drive targets because it is a simple, reliable device and the required gas pumping is manageable.

\subsection{Electrostatic Accelerator}

Electrostatic levitation has been studied at LLNL and other places. ${ }^{15-17}$ The process was called electrostatic levitation because the emphasis was on suspending the targets rather than accelerating them. Small $(800 \mu \mathrm{m}$ diameter) capsules were stably levitated in air for several days and transported in air with speeds of $10^{-4} \mathrm{~m} / \mathrm{s}$ to $0.1 \mathrm{~m} / \mathrm{s} .{ }^{15}$ As shown in Fig. 6-5, the target capsules are given an electrical charge and suspended between quadrupole rails. As described in Ref. 16, the oscillating quadrupole field provides a centering force to dynamically confine the target. As described in the following paragraphs, target position monitoring with active feedback could eliminate the need for an oscillating quadrapole field. A DC field can be added to counteract the effects of gravity. Accelerating electrodes are installed to provide the required electric field for target acceleration. There will be many accelerating electrodes in the target 
injector. The potential of each electrode should be individually controlled to provide corrections to target acceleration.

It is important that target position, velocity, and acceleration be closely monitored during the acceleration process (photodiode detectors are recommended as described in chapter 7). For example, assume the injection velocity is $100 \mathrm{~m} / \mathrm{s}$ with acceleration of $1,000 \mathrm{~m} / \mathrm{s}^{2}$. (Due to charge and electric field limitations to be discussed soon, acceleration may be limited to less than the reference case $2,000 \mathrm{~m} / \mathrm{s}^{2}$ used with other acceleration methods). The acceleration time is then $100 \mathrm{~ms}$. Position detectors could be set up to measure target position where the target is predicted to arrive each ms. Suppose the accuracy of the position measurement is $\pm 10 \mu \mathrm{m}$ in each of the three spatial dimensions and is digitally evaluated in $10 \mu \mathrm{m}$ increments. Velocity could be calculated based on two position measurements divided by the time between them. Acceleration could be calculated based on two subsequent velocity calculations. (Velocity and acceleration calculated in this manner are quite sensitive to position errors, particularly if the measurement time interval is short). Measured position, velocity, and acceleration could be compared with desired values to provide position, velocity, and acceleration errors. Appropriate weighting could be given to each of these errors, and the subsequent accelerating and suspension electric fields could be modified to correct these errors.

As an example of how this correction method could work, suppose a target had $10 \%$ less charge than was desired but electric fields were uniform and correct in the direction of acceleration and in the direction to counteract gravity and the target begins acceleration at rest by the first set of detectors. In this case the acceleration would be $900 \mathrm{~m} / \mathrm{s}^{2}$ instead of $1,000 \mathrm{~m} / \mathrm{s}^{2}$ in the acceleration direction and about $1 \mathrm{~m} / \mathrm{s}^{2}$ instead of zero in the vertical direction. About 1.11 $\mathrm{ms}$ later the target passes the second detector which is $0.5 \mathrm{~mm}$ from the first 
detector. The desired average velocity between these detectors is $0.5 \mathrm{~m} / \mathrm{s}$. The calculated velocity is $0.45 \pm 0.014 \mathrm{~m} / \mathrm{s}$. In the vertical direction the target has fallen $0.6 \mu \mathrm{m}$ which is not measurable to $\pm 10 \mu \mathrm{m}$. One could begin corrections based on the calculated velocity error or wait for another measurement to calculate acceleration error. Choosing to wait, the target passes the third detector located $1.5 \mathrm{~mm}$ from the second detector at time $2.22 \mathrm{~ms}$ instead of $2 \mathrm{~ms}$. The calculated acceleration and velocity are $10 \%$ too low $\left(900 \pm 20 \mathrm{~m} / \mathrm{s}^{2}\right.$ and $1.35 \pm 0.014 \mathrm{~m} / \mathrm{s}$ respectively). The subsequent accelerating electric fields could be increased by $11 \%$ to correct acceleration error and a small additional amount to gradually reduce the velocity error. If computations and corrections are made in the next $\mathrm{ms}$, the subsequent three measurements can be used to get new velocity and acceleration data allowing for corrections to once again be made. With this method, corrections could be made each three ms.

With $1 \mathrm{~m} / \mathrm{s}^{2}$ vertical acceleration, $4.5 \mathrm{~ms}$ is required for a target to fall $10 \mu \mathrm{m}$. So vertical motion should be detected at the fifth or sixth detector. The calculated vertical velocity would then be $0.01 \mathrm{~m} / \mathrm{s}$ and calculated acceleration $10 \mathrm{~m} / \mathrm{s}^{2}$. The actual vertical velocity and acceleration are much less. Our position measurement accuracy has substantially limited the accuracy of the vertical velocity and acceleration calculations. To avoid over correction, several measurements should be averaged before controlling transverse target position. Electric fields should be calculated as a function of position within the injector, so that the effect of target position on the forces that are exerted on the target are known and can be compensated for. With frequent and accurate position measurements, target acceleration, velocity, and position should be controllable. 


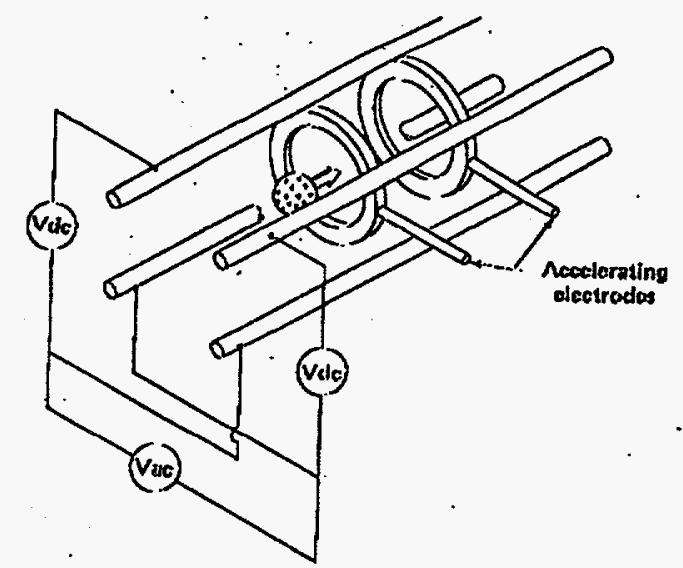

Figure 6-5. The target is dynamically confined between quadrapole rails. Alternatively, active feedback electric field control could be used to control target position and velocity. A DC field is applied to counteract gravity. Accelerating electrodes provide a traveling electric field to accelerate the target (Ref. 15).

The mass of a capsule that can be electrostatically accelerated is limited by the charge on the target and the externally applied electric field, which in turn are limited by vacuum sparking. Vacuum sparking can be initiated by field emission or field ionization. Field emission is due to tunneling of electrons through the potential barrier at the surface of the cathode under the influence of high electrostatic fields. Typically, fields of 3 to $6 \times 10^{9} \mathrm{~V} / \mathrm{m}$ are required for large scale field emission. ${ }^{18}$ Field ionization is caused by electrons tunneling away from adsorbed molecules on the surface of the anode, causing the ions to be released from the anode surface. Large scale field ionization requires even higher fields of 2 to $5 \times 10^{10} \mathrm{~V} / \mathrm{m} .{ }^{18}$ Vacuum sparking can be caused by a cascade effect with electric fields far below those required for large scale field emission. ${ }^{19}$ The cascade process assumes a localized evolution of gas from the cathode surface resulting from a few gas ions impinging on the cathode surface resulting in the release of adsorbed gas molecules and electrons. For a cascade to occur, electrons emitted from a cathode must have sufficient energy to cause ionization of gas particles enroute to the anode which in turn cause further release of electrons 
and gases from the cathode. It has been empirically determined that vacuum sparking normally does not occur if the following condition is satisfied ${ }^{19}$

$$
V E^{2} \exp \left(-\frac{1.7 \times 10^{7}}{E}\right) \leq 1.8 \times 10^{18}
$$

where $V$ is the potential (maximum energy of a charged particle at the electrode surface in $\mathrm{eV}$ prior to the spark) in volts and $E$ is the electric field at the surface of the cathode in volts per meter. Equation (6-12) is applicable for typical materials without special treatment and is known as Kilpatrick's criterion. The potential and electric fields of a charged sphere are given by

$$
E=\frac{q}{4 \pi \varepsilon_{0} r^{2}} \text { and } V=\frac{q}{4 \pi \varepsilon_{0} r}
$$

where $q$ is the charge in coulombs and $r$ is the radius in meters. Equations (6-12) and (6-13) can be combined to estimate the maximum charge that can normally be stored on a sphere of a given radius. For example, based on equations 6-12 and $6-13$, a $3 \mathrm{~mm}$ radius spherical cathode could sustain a charge of about $1.3 \mathrm{x}$ $10^{-8}$ coulombs. Based on equations $6-13$, this charge gives a field of $1.3 \times 10^{7} \mathrm{~V} / \mathrm{m}$ and a potential of $3.9 \times 10^{4} \mathrm{~V}$. Assuming an external field of $10^{6} \mathrm{~V} / \mathrm{m}$ could be applied, the force $F$ on the sphere would be given by

$$
F=E q=\left(10^{6} \mathrm{~V} / \mathrm{m}\right)\left(1.3 \times 10^{-8} \mathrm{Coul}\right)=0.013 \mathrm{~N}
$$

(The calculated electric field of $1.3 \times 10^{7} \mathrm{~V} / \mathrm{m}$ is much greater than the external field of $10^{6} \mathrm{~V} / \mathrm{m}$ so using the electric field of the sphere in Kilpatrick's criterion is approximately correct. One should verify that the electric fields at the surface of 
the external electrodes are not higher than the field on the sphere and consider the effect of the external field on the total potential difference which could cause sparking to occur.) Since $m=F / a$, the maximum mass $m$ of such a sphere would be limited by this force and the required acceleration. If $1,000 \mathrm{~m} / \mathrm{s}^{2}$ acceleration is required (half of our reference case acceleration of $2,000 \mathrm{~m} / \mathrm{s}^{2}$ ), the maximum mass would be $0.013 \mathrm{~g}$, which is a little less than a typical direct-drive target. Since field ionization requires larger fields than field emission, larger masses could be accelerated if the sphere were charged positive rather than negative. ${ }^{18}$

To provide the target charge, Ref. 15 used two plates separated by $1 \mathrm{~cm}$ with a potential difference between them. The small $800 \mu \mathrm{m}$ diameter capsules oscillated between the plates, exchanging charge at each plate. A small hole was placed in one plate through which the capsules would occasionally be propelled down the axis of the accelerator electrodes. The charge $q$ transferred by this method is given by $q=6.6 \pi \varepsilon_{0} r^{2} E$, where $r$ is the capsule radius and $E$ is the electric field between the plates..$^{20}$ For power plant target injection purposes, this procedure should be modified to control the timing of targets entering the injector and to maximize the positive charge placed on the target without vacuum sparking. As shown in Fig. 6-6, the target can be placed on a convex anode which is energized to charge the target and to start its motion into the accelerator. Using a relatively small radius anode should reduce the cathode field allowing greater charge to be placed on the target without vacuum sparking. It remains to be experimentally verified that adequate acceleration of reactor sized targets can be achieved in this manner. 


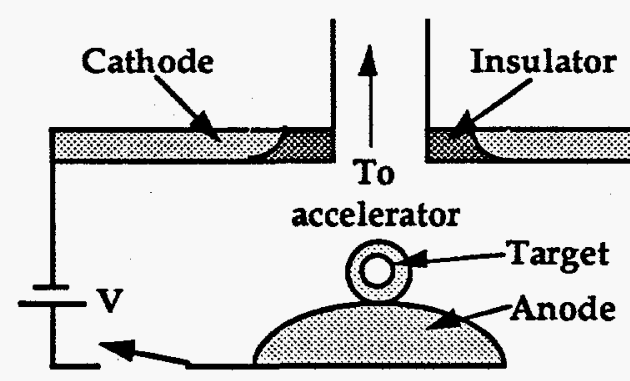

Figure 6-6. The target is placed on the anode which is then energized transferring charge to the conducting target surface. The positively charged target is then electrostatically repelled toward the accelerator.

Electrostatic acceleration has no sliding contact between the target and the accelerator. No contact implies no wear on the injector, and helps maintain the smooth surface finish of spherical direct-drive targets. Vacuum electrical breakdown may be a problem, particularly for more massive or non-spherical targets. Electrostatic acceleration shows promise primarily for accelerating low mass, spherically symmetric targets.

\subsection{Rail Gun}

The simplest electromagnetic device to accelerate a projectile is a rail gun. A rail gun is schematically illustrated in Fig. 6-7. Current flows from the power supply to one rail of the rail gun through conducting material attached to the target and back through the opposite rail to the power supply. (Many rail guns use a plasma arc behind the projectile instead of a solid conducting material. However, plasma armature rail guns have high erosion at low velocities and probably would not be suitable for target injection.) The current flowing through the rails produces a magnetic field between the rails. The interaction between this field and the current flowing through the projectile produces a force that propels the projectile down the rail gun barrel.

The amount of current required can be estimated using a simple model. Assume that the average magnetic field between the rails is equal to the magnetic 
field midway between two long cylindrical conductors. Two views of the assumed conductors together with their assumed currents and fields are shown in Fig. 6-8. The spacing between the conductors is $2 r$. The magnetic field $B$ between the conductors for a current $I$ is given by

$$
B=\frac{\mu_{0} I}{\pi r}
$$

where $\mu_{0}=4 \pi \times 10^{-7} \mathrm{~kg} \mathrm{~m} \mathrm{~s}^{-2} \mathrm{~A}^{-2}$. Assuming that the average distance that the current flows through this field is $r$, the force $F$ exerted on the conductor is given by

$$
F=\operatorname{Ir} B=\frac{\mu_{0} I^{2}}{\pi}=4 \times 10^{-7} I^{2}
$$

Solving for current gives

$$
I=\sqrt{2.5 \times 10^{6} \mathrm{~F}}=\sqrt{2.5 \times 10^{6} \mathrm{ma}}
$$

For example, the target from Fig. 1-2 with Flibe replaced by lithium has a mass of $1 \mathrm{~g}$ and radius of $7.5 \mathrm{~mm}$. To accelerate this target at $2,000 \mathrm{~m} / \mathrm{s}^{2}$ requires around $1.6 \mathrm{kA}$ and produces a field of 0.12 tesla. Since the resulting field is rather low, the required current could be reduced considerably by applying an external magnetic field of 1 tesla. Then for the same acceleration, the current would be about 26 amps. This reduced current requirement would reduce the $R R$ heating of the target and rails by two orders of magnitude. A magnetic field of order 1 tesla can easily be applied using permanent magnets. 
Target acceleration, velocity, and position can be controlled by measuring target position, calculating the target velocity and acceleration, and then varying the current to control target acceleration. This control would be much simpler than in the case of electrostatic acceleration because the gun barrel controls the targets transverse position leaving only one dimension to control with feedback.

Maintaining good electrical contact between the rails and the armature (target) in a vacuum without excessive wear may be difficult. Most conducting materials wear much faster in a vacuum than in normal atmosphere. In a vacuum the oxide layers on metals are removed causing increased friction and rapid wear. ${ }^{21}$ Graphite wears much faster in a vacuum than when 3 Torr of water vapor or atmospheric pressure of oxygen is present. ${ }^{22}$

Lubrication can reduce the wear rate substantially. The $\mathrm{MoS}_{2}$ in electrical brushes made of $88 \% \mathrm{Ag}: 12 \% \mathrm{MoS}_{2}$ provides lubrication in vacuum conditions. ${ }^{23}$ Contact lubrication experiments have typically been done with lower currents and sliding speeds than are required for a target injector. Further study is required to determine the feasibility of providing lubrication for this application. 

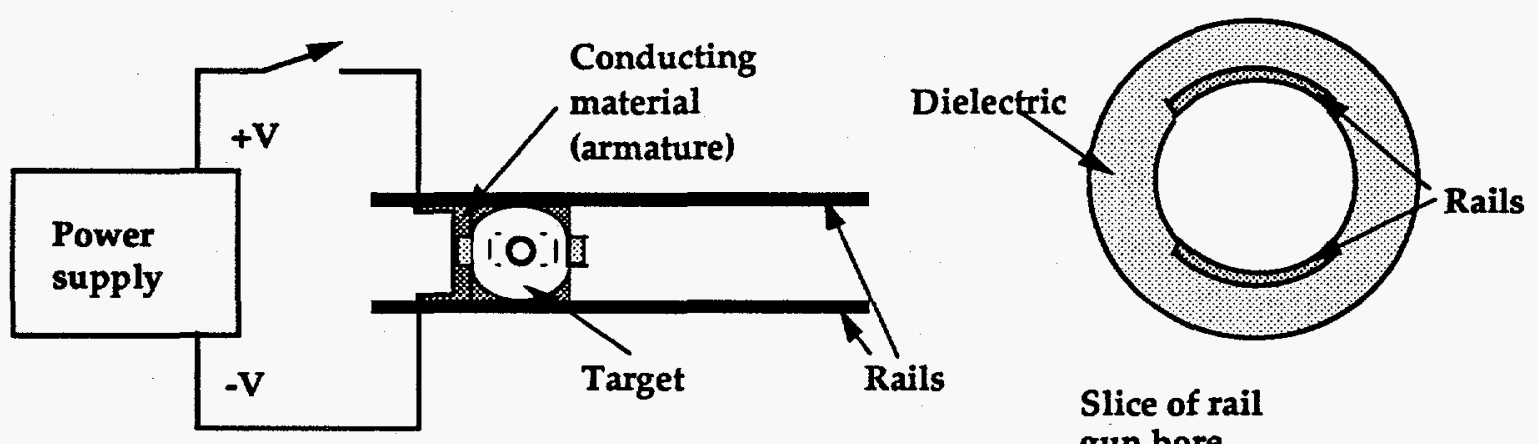

Slice of rail gun bore

Figure 6-7. Schematic of solid armature rail gun for target injection. The cylindrical shape of a typical rail gun bore is shown (other shapes may be used).
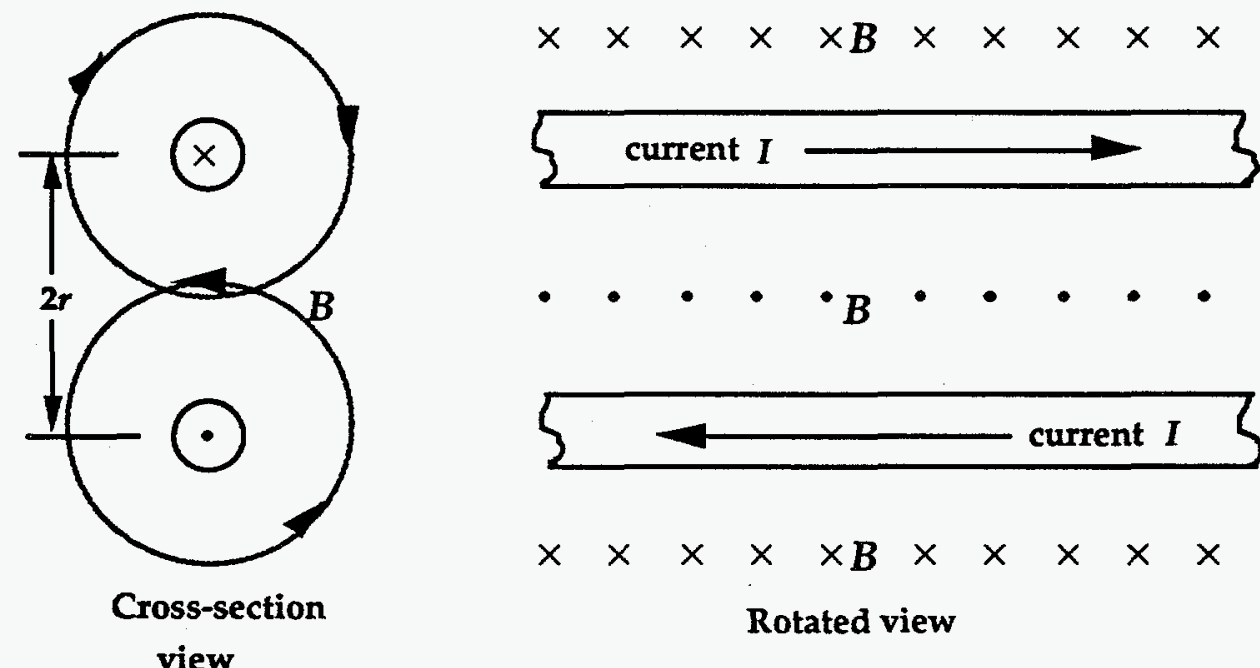

$\times \times \times \times \times B \times \times \times \times \times$

Rotated view

Figure 6-8. Simplified model to estimate the currents and fields associated with a rail gun target injector. The rails are modeled as cylindrical conductors, and the magnetic field is calculated for a point midway between the conductors. (An $X$ indicates into the page. $\mathrm{A} \bullet$ indicates out of the page.)

The decision must be made whether to send the armature into the reaction chamber with the rest of the target. For direct-drive targets, the capsule would have to be separated from the armature. For indirect-drive targets the separation may be optional. The armature could be slowed down prior to leaving the gun barrel by applying a current from the muzzle end of the injector. The target, if not 
held to the armature, could separate from the armature and proceed toward the reaction chamber. This separation of the target from the armature may adversely effect target injection accuracy. The armatures could be collected for reuse. If the conducting armatures do enter the reaction chamber, compatible materials must be used for the armature. For example, power plants using Flibe ( $\left.\mathrm{Li}_{2} \mathrm{BeF}_{4}\right)$ could use a few hundred milligrams of $\mathrm{Li}$ for the armature.

\subsection{Induction Accelerator}

Induction accelerators are more complicated than rail guns. However, they have the significant advantage of not requiring any electrical contact between the injector and the target.

D. G. Elliott provides a good introduction to traveling wave induction launchers. ${ }^{24}$ The injector consists of many closely spaced circular coils. The armature (target) contains a cylindrical conducting ring. The coils are sequentially energized to produce a magnetic field with a radial component that travels down the injector with speed $U_{S}$. Figure 6-9 shows the geometry with the coils.

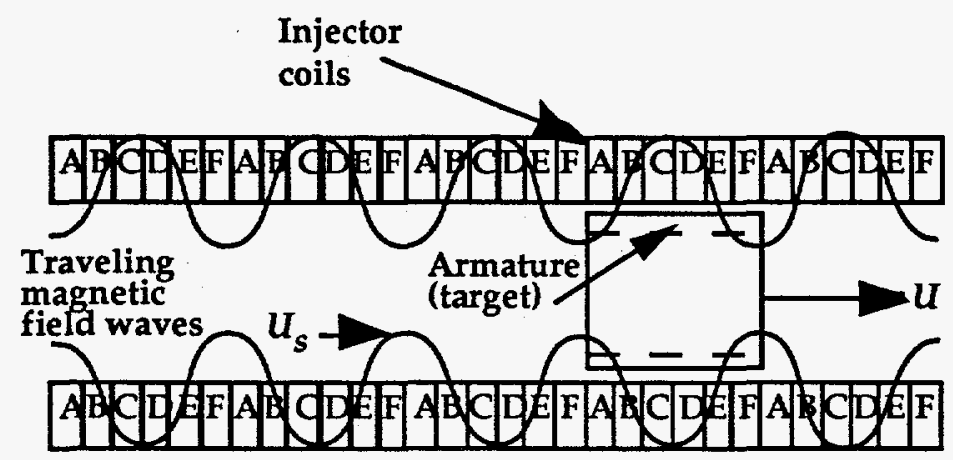

Figure 6-9. A 6-phase induction accelerator.

The traveling magnetic field passing through the armature causes a potential difference and current flow in the armature. This field can be difficult to 
calculate. It is due to the currents flowing in the coils and the induced current in the armature. The calculations can be simplified using the current sheet approximation. ${ }^{25}$ The current in the coils is modeled by a surface current $K_{b}$ at effective radius $r b(A / m) . K b$ is assumed to be a traveling sinusoidal wave of the form (in phasor notation)

$$
K_{b}=K_{b} \cos (\omega t-\beta z) \theta_{0}=\operatorname{Re}\left[\underline{K}_{b} e^{j(\omega t-\beta z)}\right] \theta_{0}
$$

where $\underline{K} b$ is the complex surface current density, $\omega$ is the angular frequency, and $\beta$ is the wave number. The induced sleeve current $\underline{K}_{S}$ is then given by ${ }^{25}$

$$
\underline{K}_{s}=\frac{-s}{\sqrt{s_{c}^{2}+s^{2}}}\left[\frac{r_{b} K_{1}\left(\beta r_{b}\right)}{r_{s} K_{1}\left(\beta r_{s}\right)}\right] \underline{K}_{b}<\phi
$$

where $s$ is the slip between the wave velocity $U_{S}$ and the sleeve speed $U, s=\left(U_{S}\right.$ $U) / U_{S} . K_{1}$ is the first order modified bessel function of the second kind, $\phi$ is the phase shift between the sleeve current sheet and the barrel current sheet and is given by

$$
\phi=\tan ^{-1} \frac{s_{c}}{s}
$$

$s_{C}$ is the critical slip at which the force is maximized for a given barrel current.

$$
S_{C}=\frac{1}{\mu_{0} \sigma_{s} a_{s} U_{s} \beta r_{s} K_{1}\left(\beta r_{s}\right) I_{1}\left(\beta r_{s}\right)}
$$

where $\mu_{0}$ is permeability of air, $a_{S}$ is the sleeve thickness, and $I_{1}$ is the first order modified bessel function of the first kind.

The average force per unit area on the sleeve $F_{z}$ is given by ${ }^{25}$ 


$$
F_{z}=\frac{s s_{c}}{s_{c}^{2}+s^{2}} \frac{\mu_{0} \beta r_{b}^{2} K_{1}^{2}\left(\beta r_{b}\right) I_{1}\left(\beta r_{s}\right)}{4 r_{s} K_{1}\left(\beta r_{s}\right)} K_{b}^{2}
$$

The electric field in the portion of the coil that is coupled to the sleeve (neglecting coil resistance) is given by ${ }^{25}$

$$
\underline{E}_{\theta}=\mu_{0} U_{s} \beta r_{b} K_{1}\left(\beta r_{b}\right) I_{1}\left(\beta r_{b}\right) K_{b}\left[\frac{s s_{c}}{s_{c}^{2}+s^{2}} \Omega+j\left(1-\frac{s^{2}}{s_{c}^{2}+s^{2}} \Omega\right)\right]
$$

where $\Omega=\frac{K_{1}\left(\beta r_{b}\right) I_{1}\left(\beta r_{s}\right)}{K_{1}\left(\beta r_{s}\right) I_{1}\left(\beta r_{b}\right)} \cdot \Omega$ is a measure of the amount of coupling between the coil and the sleeve. The field for the uncoupled portion of the coil can be obtained by setting $\Omega$ equal to zero.

Reference 25 did not specifically cover the effects of skin depth or coil resistance. Skin depth gives the effective field and current penetration distance into the surface of a conductor. Since current can flow on both sides of a sheet conductor, the effective conductor thickness for calculating resistance is roughly the lesser of the actual thickness and twice the skin depth. For good conductors the skin depth $\delta=(2 / \mu \omega \sigma)^{1 / 2} .26$ We can use this method to determine the effective thickness and resistance of both the coil and the sleeve. The resistive electric field of the coil is given by

$$
E_{\text {res }, \theta}=\frac{J}{\sigma_{b}}=\frac{K_{b}}{a_{b} \sigma_{b}}
$$

where $J$ is the current density and $a_{b}$ is the effective coil thickness. This resistive field is added to the field determined previously above.

Each coil can be composed of many turns. The current per turn is inversely proportional to the number of turns but the voltage drop is proportional to the 
number of turns. The skin effect should be mitigated by more turns but this has not yet been incorporated into the model.

We used a spreadsheet program to estimate the performance of induction launchers based on the current sheet model just presented. As an example, consider the target of Fig. 2-13 with $0.5 \mathrm{~g}$ of $\mathrm{Li}$ for sleeve material in place of the Flibe. This lithium sleeve does not contact the capsule, which should not be made of an electrical conductor to avoid direct capsule heating. Heat produced in the sleeve is insulated from the fuel by helium gas and the capsule walls. The computations are based on a uniform sleeve thickness so are only approximately correct for this target. The $1 \mathrm{~g}$ target has an outer sleeve radius of $7.5 \mathrm{~mm}$ and a length of $15 \mathrm{~mm}$. The barrel is $2.5 \mathrm{~m}$ long with a $8.4 \mathrm{~mm}$ effective radius copper coil, 6 phases, 10 turns per coil, and 4 coils per centimeter. We used room temperature material constants and assumed a wave speed of $140 \mathrm{~m} / \mathrm{s}$ requiring a frequency of $9,300 \mathrm{~Hz}$. With a current amplitude of $84 \mathrm{~A}$ and target speed of 50 $\mathrm{m} / \mathrm{s}$, the target acceleration is $2,013 \mathrm{~m} / \mathrm{s}^{2}$. The acceleration averages about 1,000 $\mathrm{m} / \mathrm{s}^{2}$ throughout the length of the barrel allowing a final target velocity of about $100 \mathrm{~m} / \mathrm{s}$. The heating of the sleeve is $194^{\circ}$ per second which for $50 \mathrm{~ms}$ of acceleration would result in an acceptable $10^{\circ}$ temperature rise. The phase voltage is $3.2 \mathrm{kV}$. The electrical power delivered to the barrel, due mostly to ohmic heating of the barrel, is $79 \mathrm{~kW}$. This is about $58 \mathrm{~W} / \mathrm{cm}^{2}$ of external barrel surface which could be removed by a cooling water jacket. The power and voltage requirements could reduced about two orders of magnitude by energizing only a small portion of the barrel that the target is in at a given time.

Induction accelerators are more complicated than rail guns but offer a significant advantage of requiring no electrical contact between the barrel and the coils. The fields even provide a centering force for the projectile within the barrel so no physical contact is necessary. A significant mass of conducting 
material must be included in the sleeve for induction acceleration to be effective. If this sleeve is to enter the reaction chamber with the capsule, it should be made of compatible materials. For example, if lithium is used for the sleeve material for a power plant containing molten Flibe, it will be necessary to provide additional fluorine to combine with the lithium.

\subsection{References}

1. R. W. Moir et al., "HYLIFE-II: A Molten-Salt Inertial Fusion Energy Power Plant Design - Final Report," Fusion Technology, 25(1), 5-25 (1994).

2. W. R. Meier et al., OSIRIS and SOMBRERO Inertial Confinement Fusion Power Plant Designs, W. J. Schafer Associates, Livermore, CA, DOE/ER/54100-1 (1992), App. A, See also R. F. Borque, W. R. Meier, and M. J. Monsler, "Overview of the OSIRIS IFE Reactor Conceptual Design", Fusion Technology, 21, 1465-1469 (1992).

3. M. J. Monsler, "Laser Fusion: An Assessment of Pellet Injection, Tracking, and Beam Pointing," Proc. Third Topical Meeting on the Technology of Controlled Nuclear Fusion, Santa Fe, NM, May 9-11, 1978, (NTIS, Springfield, VA, 1978), pp. 1186-1197.

4. R. Kreutz, "Pellet Delivery for the Conceptual Inertial Confinement Fusion Reactor HIBALL," Fusion Technology, 8, 2708 (1985).

5. L. M. Waganer et al., Inertial Fusion Energy Reactor Design Studies Final Report, McDonnell Douglas Aerospace, DOE/ER-54101 (1992), Vol. I, pp. 2-18, 2-32.

6. D. D. Ho, J. A. Harte, and M. Tabak, "Radiation-Driven Targets for Heavy Ion Fusion," Presented at the Fifteenth International Conference on Plasma Physics and Controlled Nuclear Fusion Research, Seville, Spain, Sep. 26 - Oct. 2, 1994, IAEA-CN-60/B-P-13, to be published in Nuclear Fusion.

7. R. Beeman, Air Gun Digest (DBI Books, Northfield, IL, 1977), p. 238. 
8. C. F. Taylor, The Internal Combustion Engine in Theory and Practice, 2nd ed. (M. I. T. Press, Massachusetts Institute of Technology, Boston, Massachusetts, 1979), vol. 1, pp. 173-176.

9. Ibid., vol. 2, pp. 527-529.

10. Product and Vacuum Technology Reference Book, Leybold-Heraeus Vacuum Products Inc., Export, Pa (1984), Ch. 3.

11. Sales Representative, Leybold-Heraeus Vacuum Products Inc., Export, Pa, private communication (1994).

12. G. Lewin, Fundamentals of Vacuum Science and Technology (MoGraw Hill, New York, 1965), pp. 1-20.

13. A. H. Shapiro, The Dynamics and Thermodynamics of Compressible Fluid Flow (Ronald Press, New York, 1953), vol. 1, pp. 163, 184.

14. D. J. Rose and M. Clark, Jr., Plasmas and Controlled Fusion (MIT Press and John Wiley and Sons, New York, 1961), p. 39.

15. W. L. Johnson and C. D. Hendricks, Electrostatic Levitation and Transport of Laser Fusion Targets, Lawrence Livermore National Laboratory, Livermore, CA, UCRL-83981 (1980).

16. C. D. Hendricks, Levitation, Coating , and Transport of Particulate Materials, Lawrence Livermore National Laboratory, Livermore, CA, UCRL-86117 (1981).

17. R. F. Wuerker, H. Shelton, and R. V. Langmuir, "Electrodynamic Containment of Charged Particles," Journal of Applied Physics, 30, 342 (1959).

18. R. Gomer, Field Emission and Field Ionization (Harvard University Press, Cambridge, Mass., 1961), p. 1.

19. W. D. Kilpatrick, "Criterion for Vacuum Sparking Designed to Include Both rf and dc," Review of Scientific Instruments, 28(10), 824-826 (1957). 
20. A. Y. H. Cho, "Contact Charging of Micron-Sized Particles in Intense Electric Fields," Journal of Applied Physics (USA), 35(9), 2561-4 (1964).

21. A. D. Sarkar, Wear of Metals, (Pergamon Press, New York, 1976), p. 39.

22. Ibid., p. 137.

23. F. J. Clauss, "Lubrication of Ball Bearings and Slip Rings Under High Vacuum," Proc. Symposium on Lubrication in Space, Cambridge, Mass., February 7-9, 1962 (N64-19952).

24. D. G. Elliott, "Traveling-Wave Induction Launchers," IEEE Transactions on Magnetics, 25(1), 159 (1989).

25. J. L. He, E. Levi, Z. Zabar, L. Birenbaum, and Y. Naot, "Analysis of InductionType Coilgun Performance Based on Cylindrical Current Sheet Model," IEEE Transactions on Magnetics, 27(1), 579 (1991).

26. J. D. Jackson, Classical Electrodynamics, 2nd ed. (John Wiley \& Sons, New York, 1975), p. 298. 


\section{Chapter 7 - Target Tracking and Beam Pointing}

\subsection{Target Tracking}

\subsubsection{Photodiode Timing Detectors}

Once the target leaves the injector, target tracking can begin. As illustrated in Fig. 7-1, the targets position can be detected with photodiodes. As the target passes between the light source and the photodiode, the photodiode is placed in the shadow, thereby reducing the electrical output and detecting the targets presence.
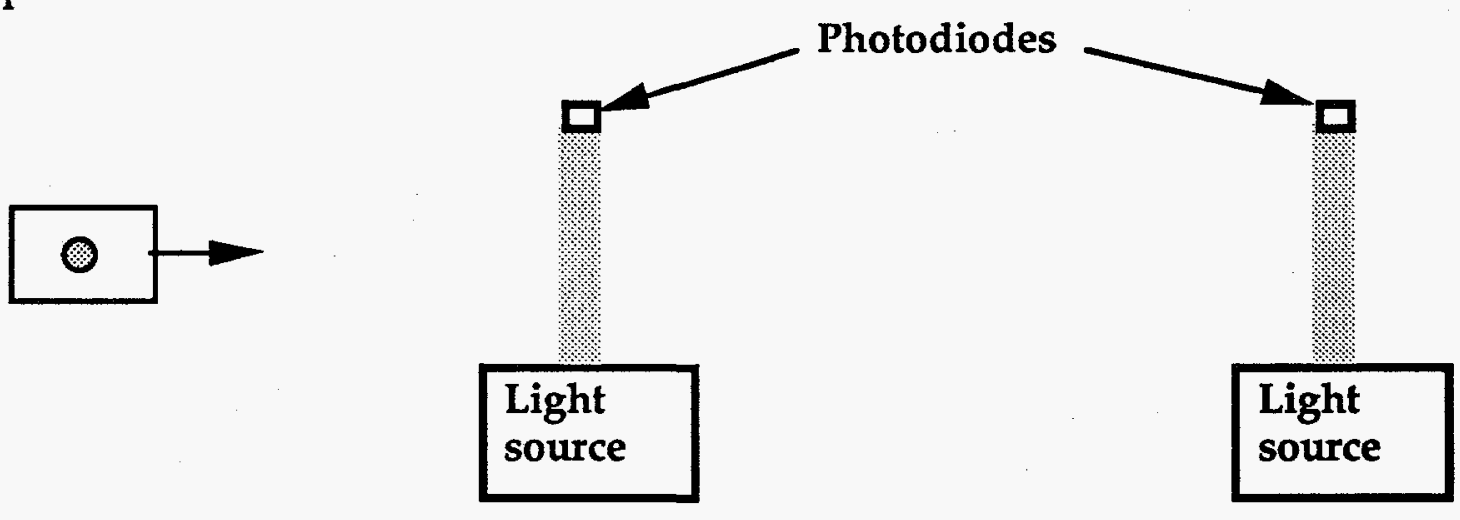

Figure 7-1. The targets position can be detected with photodiodes and light sources. Single photodiodes can be used for timing target passage by a fixed point.

\subsubsection{Detector Shielding}

The photodetectors must be well shielded from neutrons and gammas. Some types of photodiodes are more susceptible to radiation than others. For example, direct bandgap AlGaAs/GaAs photodiodes continue to perform well $^{1}$ after exposure to a high energy (in excess of $10 \mathrm{keV}$ ) neutron fluence of $10^{15} \mathrm{n} / \mathrm{cm}^{2}$ and gamma dose of $10^{6} \mathrm{~Gy}\left(10^{8} \mathrm{rad}\right)$. Their leakage current increased by less than a factor of 10, compared to typical silicon photodiodes factor of 1000 increase in leakage current. The AlGaAs/GaAs photodiodes showed a negligible decrease in responsivity, whereas silicon photodiodes 
showed a $10 \%$ to $50 \%$ drop at these doses. Since we can use rather intense light sources to illuminate our photodiode detectors, substantial degradation in photodiode performance can occur before we are no longer able to detect the target. The $10^{6} \mathrm{~Gy}$ and $10^{15} \mathrm{n} / \mathrm{cm}^{2}$ doses have been shown to be acceptable. Probably much higher (about 1 order of magnitude higher) doses could be tolerated with the AlGaAs/GaAs diodes in this application.

We now estimate the unshielded neutron dose that would be absorbed by these devices and compare it to the acceptable neutron dose noted above so that the amount of neutron shielding required can be estimated. Assume a fusion power of $3000 \mathrm{MW}$ with the detectors located 5 meters from the source. About $80 \%$ of the energy is composed of $14 \mathrm{MeV}$ neutrons. At $5 \mathrm{~m}$ distance, the neutron flux $\Phi$ is

$$
\Phi=\frac{0.8 P_{f}}{E_{n} A}=\frac{0.8\left(3 \times 10^{9} \text { Watts }\right)}{14 \mathrm{MeV} / \mathrm{n}\left(\frac{1.6 \times 10^{-13} \mathrm{~J}}{\mathrm{MeV}}\right) 4 \pi(5 \mathrm{~m})^{2}\left(\frac{100 \mathrm{~cm}}{\mathrm{~m}}\right)^{2}}=3.4 \times 10^{14} \mathrm{n} / \mathrm{cm}^{2} \mathrm{~s}
$$

The $10^{15} \mathrm{n} / \mathrm{cm}^{2}$ dose would be reached in 3 seconds. If the detectors are replaced about once a year during scheduled shutdowns, they would have to be shielded so that only about 1 in $10^{7}$ high energy neutrons gets through to the detectors. The number of high energy neutrons $n$ are reduced according to the equation

$$
\frac{n}{n_{0}}=e^{-\frac{l}{\lambda}}
$$

where $l$ is the material thickness and $\lambda$ is the transport mean free path. The natural $\log$ of $10^{7}$ is 16.1 . So roughly 16 transport mean free paths of shielding is required to reduce the neutron flux adequately. The transport mean free path for $14 \mathrm{MeV}$ neutrons in iron is $7.1 \mathrm{~cm}$ and this number tends to 
decrease with decreasing neutron energy. Using this simple model indicates that about $1.15 \mathrm{~m}$ of iron would provide adequate shielding. Actually some low $\mathrm{Z}$ shielding and neutron absorber would be more appropriate near the detectors to better attenuate lower energy neutrons. The detectors should be located in a position recessed behind shielding. The detectors can be located deep within a shield by the use of mirrors that are more radiation tolerant than the detector material. To properly calculate the shielding requirements in this problem requires computer calculations using methods such as Monte Carlo. These calculations will require significant computational time to complete since many particles must be followed, the vast majority of which do not reach the detector. This analysis is recommended for future study.

There are very few gammas produced directly in fusion reactions. Most of the gammas will be produced as a result of inelastic scattering and neutron absorption reactions. The gammas will tend to be produced throughout the shielding in decreasing numbers farther from the neutron source. As an example $3,2 \mathrm{~m}$ of ordinary concrete is required to reduce the neutron dose equivalent of $14 \mathrm{MeV}$ neutrons by 6.5 orders of magnitude (almost enough shielding to attenuate the neutrons the required 7 orders of magnitude). The capture gamma dose after passing through this much concrete is about $1.5 \times 10^{-}$ $14 \mathrm{rem} /\left(\right.$ neutron $/ \mathrm{cm}^{2}$ ). Multiplying this by the previously calculated $3.4 \times 10^{14}$ neutrons $/ \mathrm{cm}^{2} \mathrm{~s}$ unshielded at the detector gives a gamma dose of $5 \mathrm{rem} / \mathrm{s}$ or $1.6 \times 10^{6} \mathrm{~Gy} /$ year (less than twice the allowed dose rate). Since $2 \mathrm{~m}$ of concrete is almost enough shielding for both neutrons and gammas, this calculation indicates that roughly the same amount of shielding that is required for neutron shielding may also be adequate for gamma shielding. Again the detailed computations involved must are recommended for future study using transport codes. 
Due to a neutron and electromagnetic pulse, the photodiode detector readings would be unreliable immediately after the fusion micro-explosion. They could be turned off except when the targets are predicted to pass if this would help prevent detector damage.

If radiation damage or degradation due to thin film buildups on optical detectors becomes too troublesome, capacitive position detection as described in section 7.1 .5 may be used.

\subsubsection{Comparator Circuits}

The size of most small photodiodes is large compared to the required position measurement accuracy of the target. To improve the measurement precision of this method, one could mask a portion of the photodiodes surface. A better method would be to use a comparator circuit that would switch on when the photodiode amplifier voltage output drops below a preset reference value. This concept is illustrated in Fig. 7-2.

The relatively long time period over which the amplifier voltage is shown to drop is not due to the response time of the photodiode. It is simply the time it takes for the leading edge of the target to pass in front of the active area of the photodiode. Some high speed silicon photodiodes, have a response time of less than one nanosecond and an active area of diameter $0.4 \mathrm{~mm}$. At $100 \mathrm{~m} / \mathrm{s}$ a target would require $4 \mu \mathrm{s}$ to pass in front of this area. However, timing accuracy of about $10 \mathrm{~ns}$ (corresponding to target movement of about 1 $\mu \mathrm{m})$ can be achieved with a comparator circuit. 


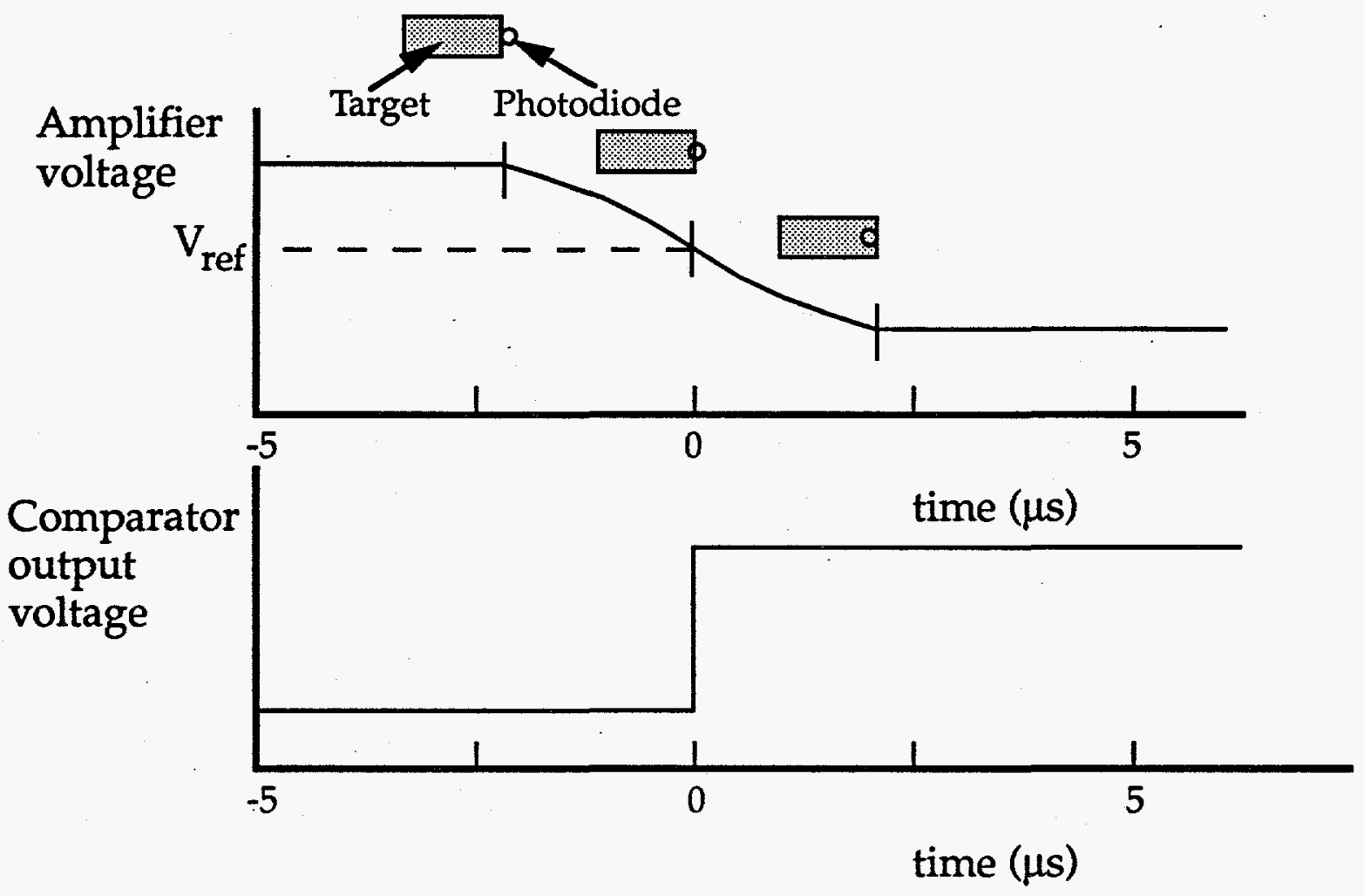

Figure 7-2. A comparator circuit can aid precise timing of a targets passage by a photodiode detector.

\subsubsection{Up/down Counters}

It is essential to predict when the target will pass through the center of the reaction chamber for timing of the driver beams to hit the target. It is possible to determine when a target will pass through the chamber center without calculations or precise knowledge of the target speed. The method requires that the detector positions and chamber center remain fixed relative to each other, or at least the ratios of the distances between them remains constant. Then, assuming constant horizontal speed, the ratio of the time required for the target to pass between the two detectors, to the time required for the target to pass between the second detector and the chamber center is a constant. With this constant ratio, an up/down counter can be used to predict target 
arrival time. When the target passes the first detector, the up/down counter begins counting up at a constant rate. When the target passes the second detector, the up/down counter begins counting down at a different rate based on the previously described distance ratio: When the counter again reaches zero, the target should be passing by the chamber center. If early warning (to fire the heavy ion driver beams for example) of the crossing is needed, some predetermined count other than zero can be used or the rate at which the counter counts down can be modified.

There are two basic types of up/down counters, analog and digital. As illustrated in Fig. 7-3, an analog up/down counter makes use of a current source and a capacitor. When the target passes the first detector, the output of a comparator circuit (as in Fig. 7-2) triggers a current source to begin charging the capacitor. When the target passes the second detector, the current source begins discharging the capacitor at an appropriate rate. Discharge current $I_{2}$ is calculated according to the following equations.

$$
V_{\max }=\frac{\left(t_{2}-t_{1}\right) I_{1}}{C}=\frac{\left(t_{3}-t_{2}\right) I_{2}}{C} \Rightarrow I_{2}=I_{1} \frac{\left(t_{2}-t_{1}\right)}{\left(t_{3}-t_{2}\right)}=I_{1} \frac{d_{1 \rightarrow 2}}{d_{2 \rightarrow 3}}
$$

When the capacitor voltage is zero, the target should be in the chamber center. A bias voltage could be used to set an appropriate time to start the driver timing sequence.

For this method to provide sufficient timing and positioning accuracy, the ratio of the charging current to the discharge current must be very consistent and the capacitor must be reset to zero voltage between each shot. The targets travel a distance of several meters with required timing repeatability corresponding to about $1 \mathrm{~mm}$ as discussed in section 2.3.2. To provide $\pm 1 \mathrm{~mm}$ 
accuracy over a distance of several meters, the system must be accurate to about \pm 1 part in $10^{4}$ (i.e. $1 \mathrm{~mm} / 10 \mathrm{~m}=10^{-4}$ ) with $\pm 10 \mu$ s timing accuracy for target speed of $100 \mathrm{~m} / \mathrm{s}$. This timing accuracy is expected to be achievable.

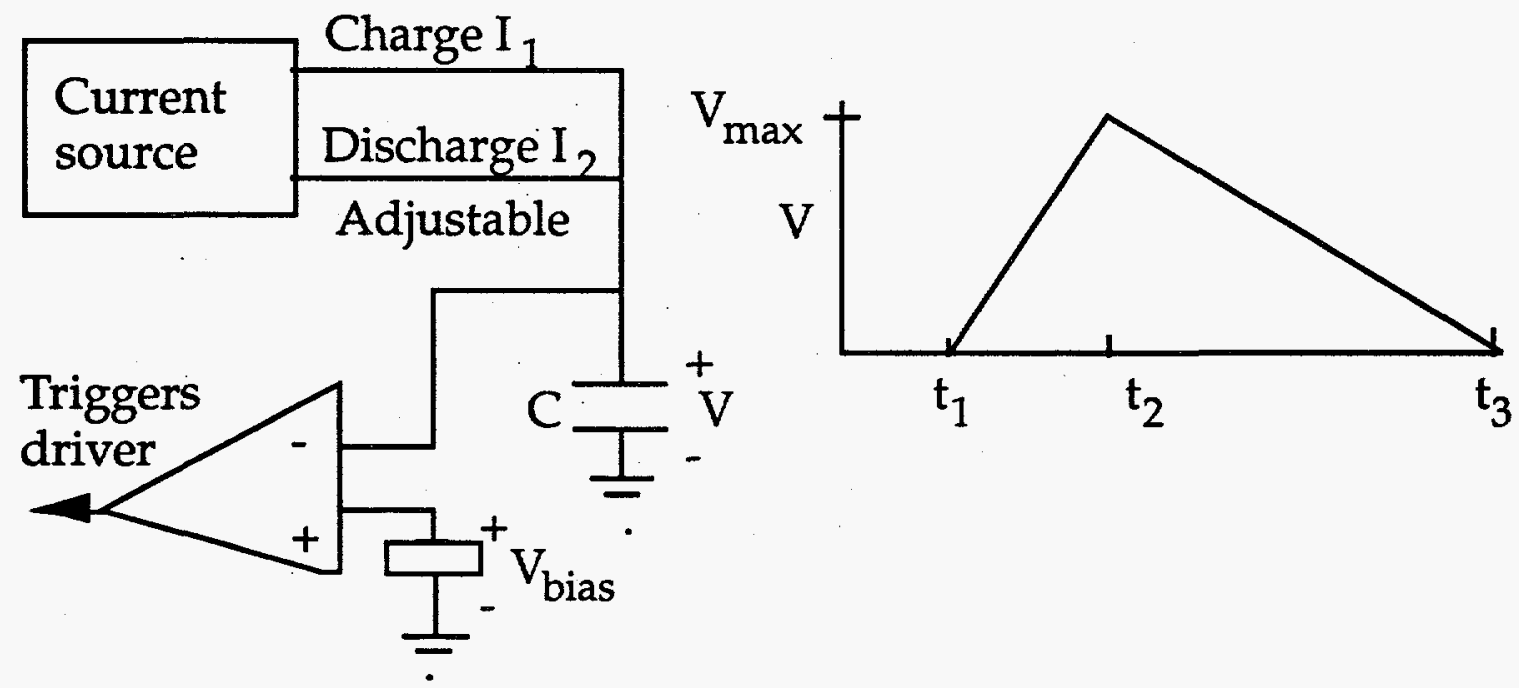

Figure 7-3. An analog up/down counter can be used to determine the firing time for the IFE driver beams.

The concept behind a digital up/down counter is illustrated in Fig. 7-4. As the target passes the first detector, the up/down counter begins counting pulses from clock number 1 operating at a frequency of $10 \mathrm{MHz}$ for example. When the target passes the second detector, the up/down counter begins counting backwards the pulses from clock number 2 operating at an appropriate different frequency determined by

$$
F r e q_{2}=F r e q_{1} \frac{d_{1 \rightarrow 2}}{d_{2 \rightarrow 3}} .
$$

When the counter is back to zero, the target should be centered in the reaction chamber. The driver timing sequence could be started at an appropriate higher number on the counter. As with the analog up/down 
counter, a high degree of repeatability is required. The ratio of the clock speeds must not vary by more than about \pm 1 part in $10^{4}$. This repeatability is expected to be more easily achievable with a digital up/down counter than with an analog up/down counter.

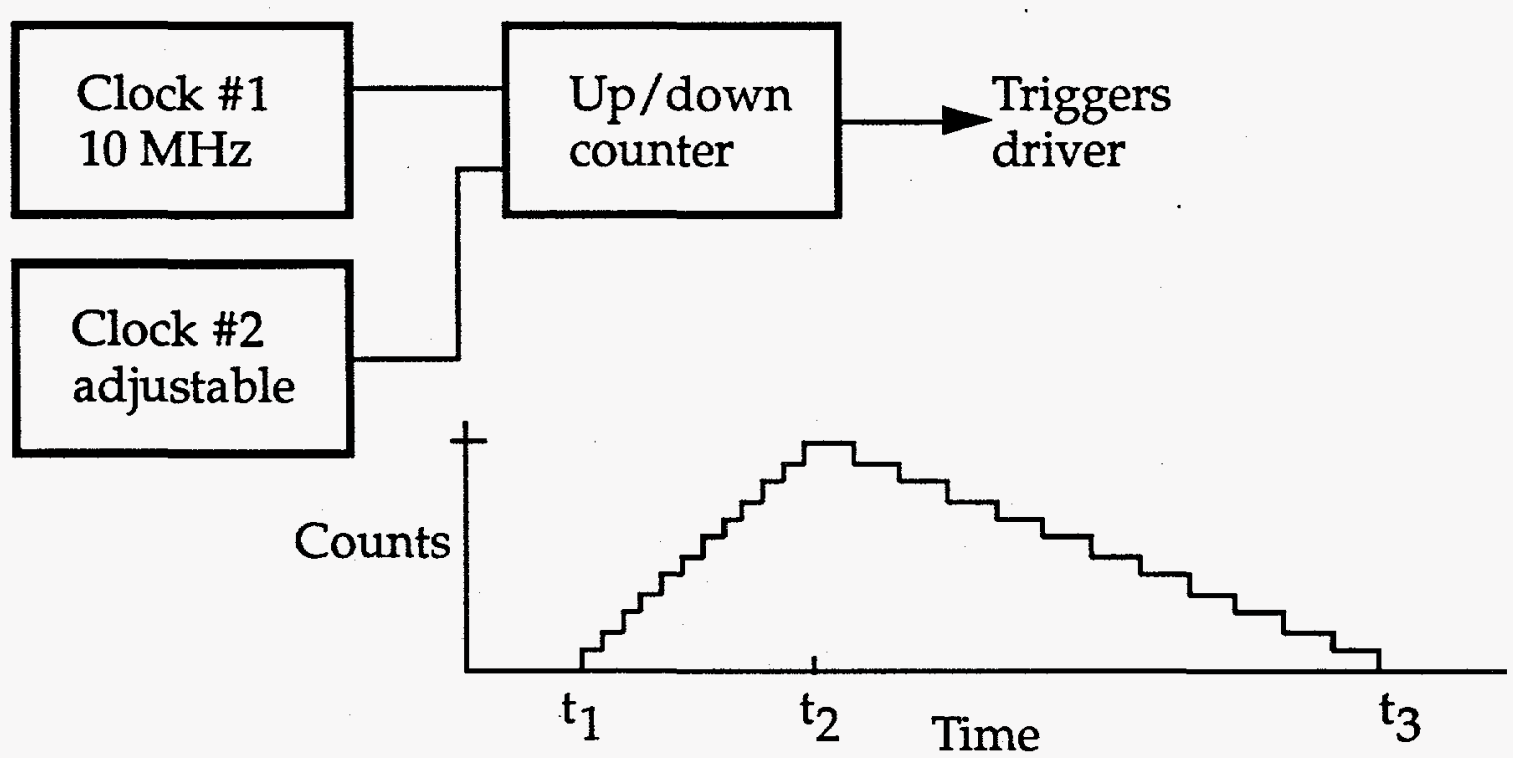

Figure 7-4. A digital up/down counter may be used to help trigger an IFE driver.

\subsubsection{Transverse Position Sensing}

To this point, we have discussed target position and timing along the direction of motion of the target. As indicated in Fig. 7-5, position measurements perpendicular to this motion can also be made with photodiode detectors. The photodiodes shown could be linear arrays of narrow diodes from which a digital output would result. Assuming the array elements were precisely positioned, the target position would be known to \pm 0.5 times the element width.

One set of two lateral position measurement arrays as shown in Fig. 7-5 should be adequate for predicting the target's final lateral position as it crosses the chamber centerline. Assume that the position of the end of the injector, 
detectors, and chamber center are constant relative to each other. Also assume that the target is traveling through a good enough vacuum so that "air drag" is negligible. Then the final deviation in the targets lateral position is directly proportional to the deviation in position at the detectors. The constant of proportionality is simply the ratio of the total flight distance to the distance to the detectors.

\section{Linear}

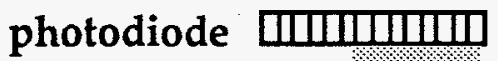
array
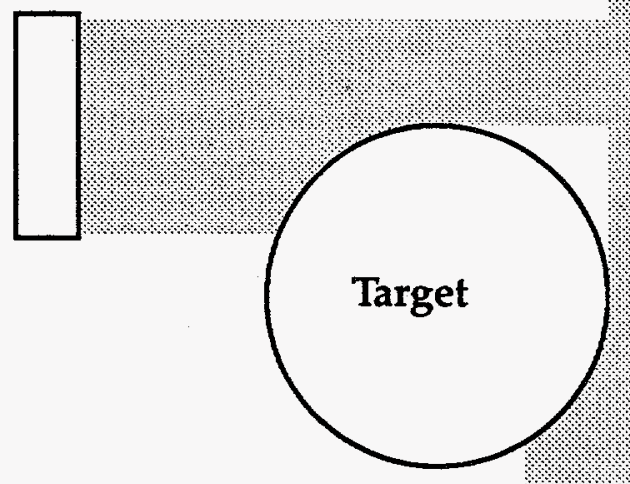

\section{Light source}

Figure 7-5. The targets lateral position is measured with linear photodiode arrays.

As discussed in the detector shielding section, photodiodes are also susceptible to radiation damage. An alternate detector which should be less sensitive to thin films and radiation damage would be a capacitive detector. A capacitive detector would consist of 4 plates with a voltage applied to each of them as shown in Fig. 7-6. When the target passes between the plates, the capacitance would be altered, and currents would flow through the ammeters as indicated in Fig. 7-6. The current flowing to each plate should be related to the target position as it passes between the plates. 


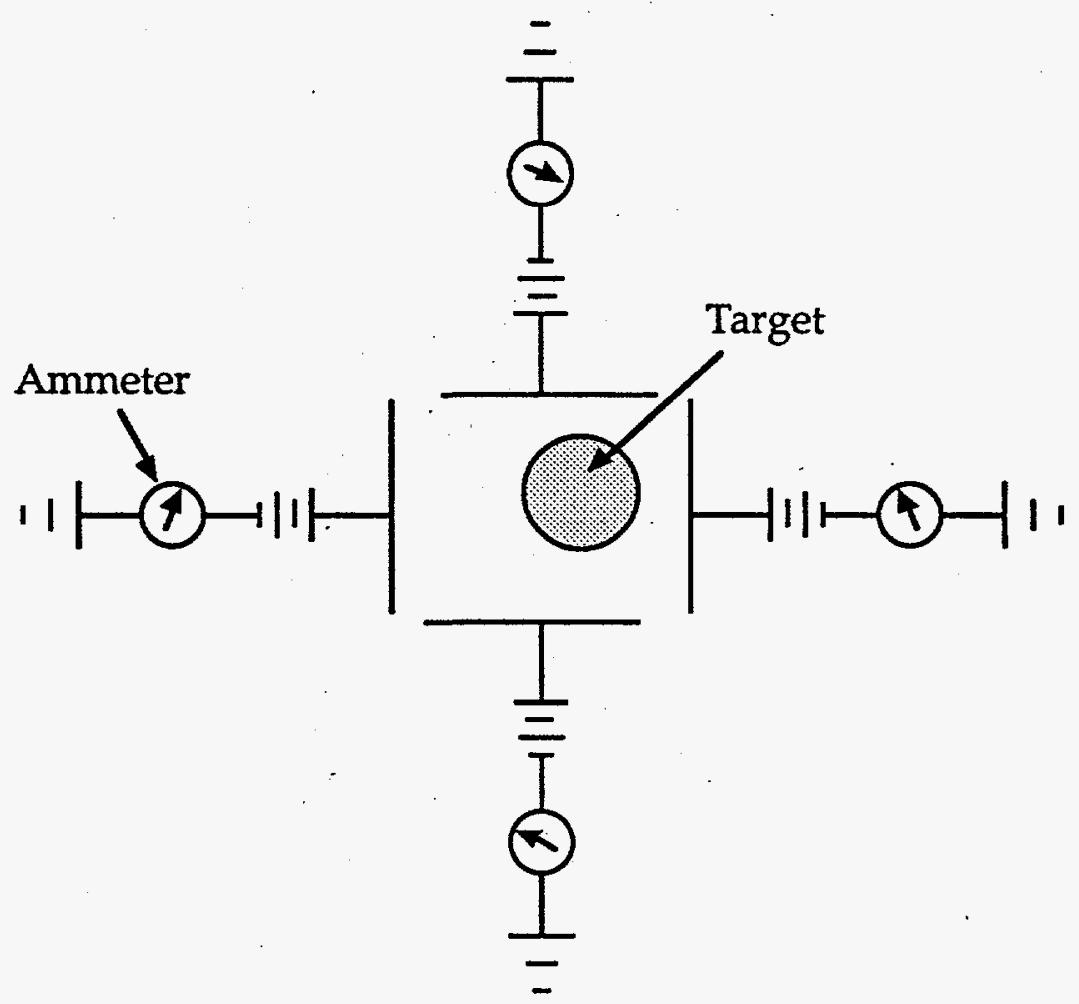

Figure 7-6. A capacitive detector may be used to sense the targets position.

\subsubsection{Target Tracking and Beam Pointing Drift}

There will likely be a gradual drift between the final target positions and the driver beam focal spot due to material expansion from heating variations, from material creep, or other factors. The targets predicted position and actual position will differ. If this drift is excessive, a feedback mechanism will be necessary to align the beams with the target positions. Measurement of this drift and an adequate feedback mechanism is recommended for future study.

\subsection{Beam Pointing}

To ensure that driver beams arrive with sufficient accuracy on target, it may be necessary to either steer the target to meet the beams or actively point the beams so that the focal point intercepts the target trajectory. Target steering is covered in chapter 5 and beam pointing is covered here. For laser beam drivers, the beam pointing can be accomplished with movable mirrors 
as described in reference 4 . For ion beam drivers, steering magnetic fields can be imposed on the driver beams to appropriately deflect the beam path.

The magnitude of the magnetic field required to steer a heavy ion beam and the amount of dispersion that is induced in the beam spot size is estimated in the following example.

\section{Example:}

Suppose that a $10 \mathrm{GeV}, 200 \mathrm{amu}$, charge 1 ion beam with $1 \%$ momentum dispersion must be deflected $10 \mathrm{~mm}$ (if active beam pointing is used, $\pm 10 \mathrm{~mm}$ is the target positioning accuracy requirement from chapter 2 , better target positioning accuracy is expected and desired.) by a $0.25 \mathrm{~m}$ long magnetic field located $5 \mathrm{~m}$ from the focal spot (see Fig. 7-7).

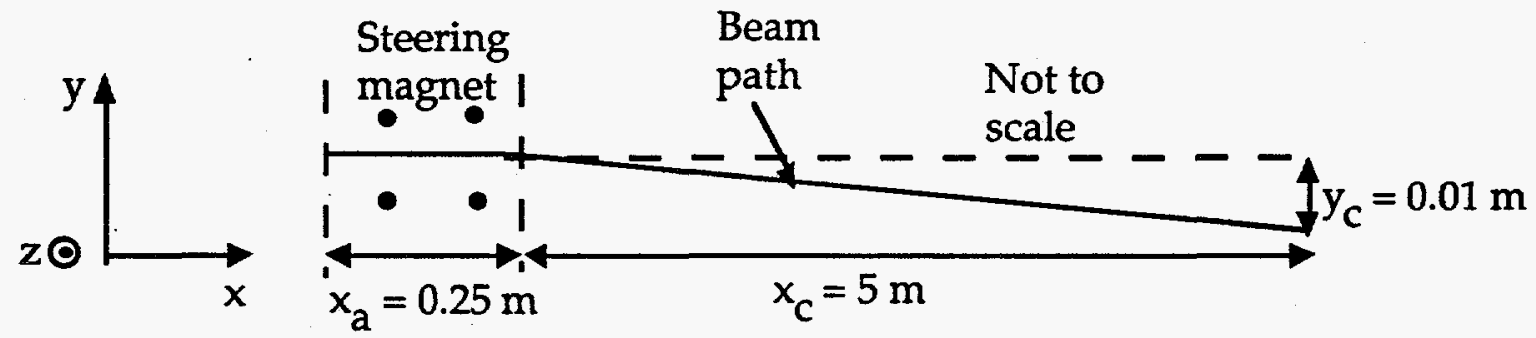

Figure 7-7. The driver beam is deflected by a magnetic field to the target position.

The speed of a $200 \mathrm{amu}, 10 \mathrm{GeV}$ particle is $1.0 \times 10^{8} \mathrm{~m} / \mathrm{s}$. To calculate the required magnetic field $B_{z}$, we make use of 2 equations for the induced velocity $v_{y}$.

$$
v_{y}=a_{y} t_{a}=\frac{q v_{x} B_{z}}{m} \frac{x_{a}}{v_{x}} \text { and } v_{y}=\frac{y_{c}}{t_{c}}=\frac{y_{c} v_{x}}{x_{c}}
$$


where $a_{y}$ is the acceleration of the ions in the magnetic field, $q$ is the ion charge, $m$ is the ion mass, $x_{a}$ is the magnetic field length, $x_{c}$ is the coasting distance past the magnetic field $\left(x_{c}>>x_{a}\right), t_{c}$ is the coasting time, and $y_{c}$ is the deflected distance at the focal point. Solving for the magnetic field gives,

$$
B_{z} \approx \frac{y_{c} v_{x} m}{x_{c} x_{a} q} \approx \frac{0.01 \mathrm{~m}\left(10^{8} \mathrm{~m} / \mathrm{s}\right)(200 \mathrm{amu})\left(\frac{1 \mathrm{~kg}}{6 \times 10^{26} \mathrm{amu}}\right)}{0.25 \mathrm{~m}(5 \mathrm{~m})\left(1.6 \times 10^{-19} \mathrm{Coul}\right)}=1.65 \mathrm{~T} .
$$

The fractional dispersion in $y_{c}$ should be approximately equal to the fractional dispersion in $v_{x}$ assuming a uniform magnetic field. Allowing for beam compression, the expected heavy ion beam speed dispersion is about $1 \% .5$ A $1 \%$ speed dispersion with a $10 \mathrm{~mm}$ steering distance would correspond to $0.1 \mathrm{~mm}$ spot size dispersion which is less than half of the required \pm 0.23 beam spot placement accuracy. Assuming the steering magnetic dipole field is made in a cylinder of radius $100 \mathrm{~mm}$, the energy stored in such a steering field is about $9 \mathrm{~kJ}$ which can be delivered to each of many driver beams in the approximately $50 \mathrm{~ms}(5 \mathrm{~m} / 100 \mathrm{~m} / \mathrm{s})$ available as the target passes from the detectors to the reaction chamber center. If most of this energy is lost, when multiplied by 6 shots per second and 12 beams for example, the average power requirement for this steering would be $648 \mathrm{~kW}$ which is significant. Assuming that target accuracy can match modern air gun accuracy (as discussed more fully in section 6.2.1), less than $1 \mathrm{~mm}$ steering should be required. Then steering would require only $0.16 \mathrm{~T}$, cause only $0.01 \mathrm{~mm}$ spot dispersion, and the field energy would only be about $90 \mathrm{~J}$.

\subsection{References}

1. J. J. Wiczer, C. E. Barnes, T. A. Fischer, L. R. Dawson, and T. E. Zipperian, 
"AlGaAs/ GaAs Radiation Hardened Photodiodes," Proceedings of SPIE (International Society of Optical Engineering), Volume 506, Fiber Optics in Adverse Environments II (1984).

2. E. F. Plechaty, D. E. Cullen, R. J. Howerton, and J. R. Kimlinger, Tabular and Graphical Presentation of 175 Neutron Group Constants Derived from the LLL Evaluated Neutron Data Library (ENDL), Lawrence Livermore National Laboratory, Livermore, CA, UCRL-50400 (1976), Vol.16 .

3. A. E. Profio, Radiation Shielding and Dosimetry, (John Wiley \& Sons, New York, 1979), p. 440.

4. M. J. Monsler, "Laser Fusion: An Assessment of Pellet Injection, Tracking, and Beam Pointing," Proc. Third Topical Meeting on the Technology of Controlled Nuclear Fusion, Santa Fe, NM, May 9-11, 1978, (NTIS, Springfield, VA, 1978), pp. 1186-1197.

5. R. O. Bangerter, Lawrence Berkeley Laboratory, Berkeley, CA, private communication (1992). 


\section{Chapter 8 - Summary, Conclusions, and Recommendations}

\subsection{General}

Inertial fusion energy (IFE) is a potential source of nearly unlimited, environmentally benign electrical power. A significant effort is underway to demonstrate the scientific feasibility of producing high gain in the laboratory which will be necessary for IFE power production. To make use of this energy, assuming these efforts are successful, IFE power plants must be designed and constructed which economically transform this pulsing explosive energy into electrical power. Target injection, tracking, and beam pointing are essential to delivering and igniting the fuel in an IFE power plant. Many interesting and challenging problems must be overcome. This dissertation has identified and provided solutions to many of the problems in the areas of target injection, tracking, and beam pointing.

\subsection{Requirements}

The interdependent relationships between target acceleration $\left(1,800 \mathrm{~m} / \mathrm{s}^{2}\right.$ for a $0.1 \mu \mathrm{m}$ thick membrane), target speed $(100 \mathrm{~m} / \mathrm{s})$, reaction chamber size ( 3 $m$ radius), target injector (acceleration) length $(3 \mathrm{~m})$, target coasting distance (5 $\mathrm{m})$, and interpulse time ( $0.17 \mathrm{~s})$ were described analytically and graphically (nominal values are given in parentheses).

The minimum target injection speed consistent with power plant pulse rate, chamber conditions, and target heating should normally be chosen for minimum injector length and ease of target tracking. Even if one target injector can provide targets at the desired target speed and pulse rate, two target injectors, each operating at one half capacity should be used to provide redundancy and therefore improve plant availability. 
Accurate placement $(\sim \pm 0.4 \mathrm{~mm})$ of driver beams on target is required to achieve high gain. This requires precise target tracking and beam pointing and limits allowed target tumble.

Prior to injection, the targets are cooled in cryogenic helium. During injection the target fuel temperature must be kept well below the triple point of $19.7 \mathrm{~K}$ (perhaps about $17.4 \mathrm{~K}$ for adequate mechanical strength). We assumed as a reference case that fuel temperature could increase only from 17.3 to $17.5 \mathrm{~K}$. Conduction target heating was found to be sufficiently slow for indirect-drive targets.

Target injection requirements are pretty similar for direct-drive and indirect-drive targets. Some differences between the two follow. Direct-drive targets heat much more rapidly and may require higher injection speed so that less time is spent in the hot reaction chamber. It may be useful to put a thin reflective outer surface on the capsule to minimize radiative heat flux on direct-drive targets. Direct drive targets also require very smooth surface finishes which must be protected during the injection process. These and other differences have caused us to recommend a different injection method for direct-drive than for indirect-drive targets.

Target design work is still occurring. Minor changes in target design will certainly occur. The methods used in this dissertation should be applicable to minor changes in target design. For example, many of the methods presented here are applicable to different size and mass targets. Major design changes are also possible and we will have to consider the implications of these changes as they occur.

Table 2-1 summarizes typical target injection goals and requirements. Attainment of these requirements may be achievable but substantial further study and development is necessary. 


\subsection{Structural Limits on Acceleration}

Large target acceleration is desirable, since it allows shorter target injectors and higher pulse rates, but will probably be limited by target structural considerations. The structural acceleration limits were investigated in chapter 3 and 4. A dual membrane support of IFE capsules within a hohlraum is proposed. $1840 \mathrm{~m} / \mathrm{s}^{2}$ acceleration of a typical $2.4 \mathrm{~mm}$ radius $30 \mathrm{mg}$ capsule is achievable with 0.1 micron thick membranes.

Expected capsule oscillation frequency in the hohlraum is in the kilohertz range and approximate values can be easily calculated. Potential problems arising from vibrations can be minimized in two ways. The vibration amplitude can be reduced by factor of 2 by prestressing membranes to one half of the design stress. The amplitude may be reduced by an additional order of magnitude (to order of $10 \mu \mathrm{m}$ ) by increasing and decreasing the target acceleration gradually over a few target oscillation periods.

Membrane support of IFE capsules may be useful if the membrane does not excessively absorb driver energy and affect capsule implosion symmetry. If membrane support is used, the dual membrane design is recommended. Substantially greater target acceleration and reduced capsule displacement result from this geometry.

Additional study is required to quantify the effects of the membrane on target implosion symmetry and hence on target energy gain. The question of how thick the membrane can be should be investigated. However, a $0.1 \mu \mathrm{m}$ thick membrane is expected to have little effect on target gain. The distance that a capsule may be off center in a hohlraum and retain high gain must be quantified but will probably not be difficult to meet. A method for manufacturing the targets with properly prestressed membranes should be developed. 
Target fuel strength is adequate for accelerations of order $10,000 \mathrm{~m} / \mathrm{s}^{2}$ if temperature is held below about $17.4 \mathrm{~K}$.

\subsection{Target Steering}

We noted that target steering may be necessary to provide sufficient target injection accuracy. One method of steering is to provide a target with a positive charge and alter the course with an electric field applied between steering electrodes. This method should be effective for steering light weight, spherical, direct-drive targets.

We also investigated, using computational methods, the possibility of steering non spherical indirect-drive targets with electric fields. When a nonspherical target passes between the steering electrodes with a small rotation angle, a torque results on the target. We found that for typically sized targets, and reasonably accurate injectors requiring small steering distances that this torque would not cause excessive target rotation.

\subsection{Target Injection Methods}

Different target injection methods are probably appropriate for different target and power plant designs.

A gas gun is recommended for targets (such as typical indirect drive targets) that can support a gas pressure load on one end and can slide along the gun barrel without damage. For other types of targets, a sabot would be necessary. The detachment of the sabot from the target may exert an impulse to the target. This impulse could degrade target accuracy. The amount of gas required for each target (about 10 to $100 \mathrm{mg}$ ) is acceptable.

An electrostatic accelerator injection system is recommended for use with low mass spherical direct drive targets. No additional materials must be added to the target for propulsion. Since there is no physical contact between 
the target and the injector there will be no wear of either component during the injection process. However, this lack of physical contact requires feedback control of the target position which must be developed and demonstrated.

An induction accelerator has an advantage of no electrical contact between the target and the injector. Physical contact is not even necessary, so the wear should be minimal. It requires conductive material for the target sleeve ( $0.5 \mathrm{~g}$ in the example) which would be a disadvantage for some power plant designs.

A rail gun may be useful for target injection and is a simpler device than an electrostatic accelerator or induction accelerator. It requires electrical contact between the target and the rails and may have a significant wear rate.

Each of these injection methods have advantages and disadvantages. They all have the possibility of working well with certain types of targets. However, the gas gun is the simplest of the devices and appears to have the lowest risk of failure. The gas gun will probably work well with indirect drive heavy ion beam targets as used in HYLIFE-II. The ability of a target injection system to reliably meet target injection requirements should be experimentally verified.

\subsection{Target Tracking and Beam Pointing}

Target tracking can be achieved with photodiodes or capacitive detectors. Up/down counters can be used to help time the delivery of driver energy. Target steering or active beam pointing will be necessary if injected target position is not adequately repeatable.

\subsection{Recommendations for Future Work}

An experimental injector (such as described in the next section) should be built to demonstrate accurate target injection placement and tracking with 
required target speed, acceleration, and pulse rate. A small scale (low energy) driver should then be incorporated to verify the feasibility of repeatedly hitting the targets accurately on the fly. A method of providing feedback on the accuracy of hitting targets must be developed so that gradual drift in beam placement accuracy can be corrected. Cryogenic target handling methods for target injection will also have to be developed. The effect of membrane thickness capsule position error on capsule implosion symmetry should be investigated. Convection heating of indirect-drive targets requires improved modeling and computation. Methods of reducing direct drive capsule heating should be further investigated.

\subsubsection{Proposed Target Injection and Tracking Experiment}

An experiment to use a helium gas gun to accelerate non-cryogenic "targets" at pulse rates, distances, and speeds comparable to those found in an operating power plant is recommended to be carried out. The experiment would measure target positions using photodiode detectors, then predict and verify subsequent target positions. Such an experiment could remove doubt as to the accuracy of injected targets and predicted arrival times and positions.

We proposed such an experiment to the U.S. Department of Energy, Office of Fusion Energy. ${ }^{1}$ We proposed to build a helium gas gun with a revolver loading mechanism. The surrogate "targets" will be shot into an $8 \mathrm{~m}$ long, 0.4 m diameter vacuum chamber with the gas flow controlled by fast opening and shutting solenoid operated valves. 6 shots will be fired with their positions tracked and predicted in one second. The amount requested from DOE for the entire project is $\$ 212,603$. 


\subsection{References}

1. P. F. Peterson and V. E. Schrock, Experimental Investigation and Demonstration of IFE Target Injection and Tracking (Research Proposal written by R. W. Petzoldt), University of California, Berkeley, CA (1993). 


\section{Selected Bibliography}

Literature referenced in this dissertation are indicated with an asterisk.

Afanas'ev, Y. V. et al., "Acceleration and Compression of Matter Due to Laser Heating and Ablation," Laser and Particle Beams, 6(1), 1-23 (1988).

Afanas'ev, Y. V. et al., "Stationary Model of the "Corona" of Spherical Laser Targets," Soviet Physics JETP, 44(2), 311-316 (1976).

Alston, L. L., ed., High Voltage Technology, (Oxford University Press, London, 1968).

Barber, J. P., and C. L. McDonald, "The Mechanical Design of Armatures,"

IEEE Transactions on Magnetics, 25(1), 79-82 (1989).

Barnes, C. E., and J. J. Wiczer, Radiation Effects in Optoelectronic Devices,

Sandia National Laboratory, Albuquerque, NM, Sand 84-0771 (1984).

Beeman, R., Air Gun Digest (DBI Books, Northfield, IL, 1977).*

Borque, R. F., W. R. Meier, and M. J. Monsler, "Overview of the Osiris IFE

Reactor Conceptual Design", Fusion Technology, 21, 1465-1469 (1992).

Cho, A. Y. H., "Contact Charging of Micron-Sized Particles in Intense Electric Fields," Journal of Applied Physics (USA), 35(9), 2561-2564 (1964).*

Clauss, F. J., "Lubrication of Ball Bearings and Slip Rings Under High

Vacuum," Proc. Symposium on Lubrication in Space, Cambridge, Mass.,

February 7-9, 1962 (N64-19952).*

Damm, C. C. et al., Target-Plasma Production by Laser Irradiation of a Pellet in

the Baseball II-T Experiment, Lawrence Livermore National Laboratory,

Livermore, CA, UCRL-52363 (1977).

Devaney, J. J., Injection of Laser Fusion Pellets, Part 1, Accuracy Required, Acceleration, and Residual Gas Deflections, Los Alamos Scientific Laboratory, Los Alamos, NM, LA-7477-MS (1978). 
Driga, M. D., W. F. Weldon, and H. H. Woodson, "Electromagnetic Induction Launchers," IEEE Transactions on Magnetics, 22(6), 1453-1458 (1986).

Driga, M. D., and W. F. Weldon, "Induction Launcher Design

Considerations," IEEE Transactions on Magnetics, 25(1), 153-158 (1989).

Elliott, D. G., "Traveling-Wave Induction Launchers," IEEE Transactions on Magnetics, 25(1), 159-163 (1989).*

Englemann, B., NIKE2D A Nonlinear, Implicit, Two-Dimensional Finite

Element Code for Solid Mechanics - User Manual, Lawrence Livermore National Laboratory, Livermore, CA, UCRL-MA-105413 (1991).

Erin, T., and C. D. Hendricks, "Uniform Charged Solid Particle Production," Review of Scientific Instruments, 39(9), 1269-1271 (1968).

Evans, B. L., and P. A. Young, "Optical Absorption and Dispersion in Molybdenum Disulfide," Proceedings of the Royal Society, 284, 415 (1965). Ferrentino, G. L., and W. J. Kolkert, "On the Design of an Integrated Metal Armature and Sabot for Railguns," IEEE Transactions on Magnetics, 22(6) (1986).

Franklin, G. F., J. D. Powell, and M. L. Workman, Digital Control of Dynamic Systems, 2nd. ed. (Addison-Wesley, New York, 1992).

Franklin, G. F., J. D. Powell, and A. Emami-Naeini, Feedback Control of Dynamic Systems (Addison-Wesley, New York, 1986).

Friebele, E. J. et al., "Inter Laboratory Comparison of Radiation-Induced Attenuation in Optical Fibers. Part I: Steady State Exposures," Journal of Lightwave Technology, 6(2) (1988).

Friebele, E. J. et al., "Optical Fiber Waveguides in Radiation Environments, II," Nuclear Instruments and Methods in Physics Research, B1, 355-369 (1984). 
Fuller, R. L., J. R. Kitzmiller, and M. W. Ingram, "Design and Testing of a 0.60 Caliber, Augmented Railgun," IEEE Transactions on Magnetics, 27(1), 45-49 (1991).

Glossbrenner, E. W., and B. K. Witherspoon, "Brushes Containing Molybdenum Disulfide for Slip Rings in Spacecraft Application," Proc.

Thirty Third Meeting of the IEEE Holm Conference on Electrical Contacts, Chicago, IL, Sep. 21-23, 1987 (The Palmer House, New York, 1987).

Glumb, R. J., and H. Krier, "Concepts and Status of Laser-Supported Rocket Propulsion," Journal of Spacecraft, 21(1), 70-79 (1984).

Gomer, R., Field Emission and Field Ionization, (Harvard University Press, Cambridge, Mass., 1961).*

Green, A. E., and J. E. Adkins, Large Elastic Deformations, 2nd ed. (Oxford Univ. Press, London, England (1970).*

Haas, R. A., General Principals of Inertial Confinement Fusion, Lawrence Livermore National Laboratory, Livermore, CA, UCRL-88709 (1983).* Hallquist, J. O., MAZE - An Input Generator for DYNA2D and NIKE 2D, Lawrence Livermore National Laboratory, Livermore, CA, UCID-19029, Rev. 2 (1983).

Hallquist, J. O., and J. L. Levatin, ORION: An Interactive Color Post- Processor for Two-Dimensional Finite Element Codes, Lawrence Livermore National Laboratory, Livermore, CA, UCID-19310, Rev. 2 (1985).

Hawke, R. S., "Fusion Fuel Pellet Injection with a Railgun," Journal of Vacuum Science Technology, 1(2), 969-973 (1983).

Hawley, R., "Vacuum as an Insulator," Vacuum, 10, 310-317 (1960).

He, J. L. et al., "Analysis of Induction-Type Coilgun Performance Based on Cylindrical Current Sheet Model," IEEE Transactions on Magnetics, 27(1), 579 (1991).* 
Hendricks, C. D., and W. L. Johnson, Electrostatic Levitation, Control and Transport in High Rate, Low Cost Production of Inertial Confinement

Fusion Targets, Lawrence Livermore National Laboratory, Livermore, CA, UCRL-82752 (1979).

Hendricks, C. D., Levitation, Coating, and Transport of Particulate Materials, Lawrence Livermore National Laboratory, Livermore, CA, UCRL-86117 (1981).*

Hermann, A. M., "Electrical Resistivity of Intercalated Molybdenum

Disulfide," Solid State Communications, 13, 1065-1068, (1973).

Hibbs, S. M. et al., "A Fuel Pellet Injector for the Microwave Tokamak

Experiment," Journal of Vacuum Science and Technology, 9(3), 835-841 (1991).

Ho, D. D., J. A. Harte, and M. Tabak, "Radiation-Driven Targets for Heavy-Ion Fusion," Presented at the Fifteenth International Conference on Plasma Physics and Controlled Nuclear Fusion Research, Seville, Spain, Sep. 26 Oct. 1, 1994, IAEA-CN-60/ B-P-13.*

Holm, R., and E. Holm, Electric Contacts Handbook, 3rd ed. (Springer, Berlin, 1958).

Incropera, F. P., and D. P. DeWitt, Fundamentals of Heat Transfer, (John Wiley \& Sons, New York, 1991).

Inertial Confinement Fusion Central Station Electric Power Generating Plant, Final Report, U. S. Department of Energy, DOE/DP/40086-1 (1981).* Jackson, J. D., Classical Electrodynamics, 2nd ed. (John Wiley \& Sons, New York, 1975).*

Jakob, M., Heat Transfer (John Wiley \& Sons, New York, 1941).* 
Johnson, W. L., and C. D. Hendricks, Electrostatic Levitation and Transport of

Laser Fusion Targets, Lawrence Livermore National Laboratory,

Livermore, CA, UCRL-83981 (1980).*

Kilpatrick, W. D., "Criterion for Vacuum Sparking Designed to Include Both rf and dc," Review of Scientific Instruments, 28(10), 824-826 (1957).*

Kitzmiller, J. R. et al., "Final Design of an Air Core, Compulsator Driven, 60

Caliber Railgun System," IEEE Transactions on Magnetics, 27(1), 50-55 (1991).

Klein, S. A., W. A. Beckman, and G. E. Meyers, FEHT A Finite Element Analysis Program (F-Chart Software, Middleton, WI, 1991).*

Korde, R. et al., "The Effect of Neutron Irradiation on Silicon Photodiodes," IEEE Transactions on Nuclear Science, 36(6), 2169-2175 (1989).

Koresheva, E. R. et al., "The Peculiarities of Laser Cryogenic Target Destruction, and their Injection into a Powerful Laser Focus," Laser and Particle Beams, 6(2), 245-253 (1988).

Kovarik, V. et al., "The Modernization and Improvement of the BNL Short Separators," Nuclear Instruments and Methods, 158, 371-376 (1979).

Kreutz, R., "Pellet Delivery for the Conceptual Inertial Confinement Fusion Reactor HIBALL," Fusion Technology, 8, 2708 (1985).*

Lamb, H., Hydrodynamics, 6th ed. (Dover Publications, New York, 1932), p. 590.

Landau, L. D., and E. M. Lifshitz, Mechanics, 3rd ed. (Pergamon Press, Oxford, 1976).*

Lewin, G., Fundamentals of Vacuum Science and Technology (McGraw Hill, New York, 1965).*

Lindl, J. D. et al., Review of Target Studies for Heavy Ion Fusion, Lawrence Livermore National Laboratory, Livermore, CA, UCRL-94382 (1986). 
Lischka, H. et al., "Radiation Sensitivity of Light Emitting Diodes (LED), Laser Diodes (LD), and Photodiodes (PD)," IEEE Transactions on Nuclear Science, 39(3), 423-427 (1992).

McCarville, T. J. et al., SAFIRE-A Systems Analysis Code for ICF Reactor Economics, Lawrence Livermore National Laboratory, Livermore, CA, UCRL-15872 (1987).*

McCracken, D. D., and W. I. Salmon, Computing for Engineers and Scientists with FORTRAN 77, 2nd ed. (John Wiley \& Sons, New York, 1988).

Meier, W. R. et al., OSIRIS and SOMBRERO Inertial Confinement Fusion Power Plant Designs, W. J. Schafer Associates, Livermore, CA, DOE/ER/54100-1 (1992).*

Moir, R. W. et al., "HYLIFE-II: A Molten-Salt Inertial Fusion Energy Power Plant Design - Final Report," Fusion Technology, 25(1), 5-25 (1994).*

Monsler, M. J., "Laser Fusion: An Assessment of Pellet Injection, Tracking, and Beam Pointing," Proc. Third Topical Meeting on the Technology of Controlled Nuclear Fusion, Santa Fe, NM, May 9-11, 1978 (NTIS, Springfield, VA, 1978), pp. 1186-1197.*

Mortenson, W. K., Microcomputer-based Pellet Trajectory Guidance System for the Baseball II-T Experiment, Lawrence Livermore National Laboratory, Livermore, CA, UCRL-79685 (1977).

Nuckolls, J. et al., "Laser Compression of Matter to Super-High Densities: Thermonuclear (CTR) Applications," Nature, 239, 139-142 (1972).

Onuki, T., and E. R. Laithwaite, "Optimized Design of Linear-InductionMotor Accelerators," Proc. IEE, 118, 349-355 (1971).

O'Shea, D. C., and D. C. Peckham, "Resource Letter L-1: Lasers," American Journal of Physics, 49(10), 915-925 (1981).

Peterson, P. F., and V. E. Schrock, Experimental Investigation and 
Demonstration of IFE Target Injection and Tracking (Research Proposal written by R. W. Petzoldt), University of California, Berkeley, CA (1993).* Petzoldt, R. W., "Oscillating Liquid Flow ICF Reactor," Fusion Technology, 19(3), 758-762 (1991).

Petzoldt, R. W., and R. W. Moir, Target Injection Methods for Inertial Fusion Energy, Lawrence Livermore National Laboratory, Livermore, CA, UCRLJC-116030 (1994) (To be published in the Proceedings of the Eleventh Topical Meeting on the Technology of Fusion Energy held in New Orleans LA, June 19-23, 1994).

Petzoldt, R. W., and R. W. Moir, Membrane Support of Accelerated Fuel Capsules for Inertial Fusion Energy Reactors, Lawrence Livermore National Laboratory, Livermore, CA, UCRL-ID-114020 (1993).*

Pitts, J. H. et al., The Cascade Inertial Confinement Fusion Reactor Concept, Lawrence Livermore National Laboratory, Livermore, CA, UCRL-LR104546 (1990).*

Pitts, J. H., Heat Limitations on Pellets Used in ICF Reaction Chambers, Lawrence Livermore National Laboratory, Livermore, CA, UCRL-85975 (1981).*

Plechaty, E. F. et al., Tabular and Graphical Presentation of 175 Neutron Group Constants Derived from the LLL Evaluated Neutron Data Library (ENDL), Lawrence Livermore National Laboratory, Livermore, CA, UCRL50400 (1976), Vol.16.*

Product and Vacuum Technology Reference Book, Leybold-Heraeus Vacuum Products Inc., Export, $\mathrm{Pa}(1984) . *$

Profio, A. E., Radiation Shielding and Dosimetry, (John Wiley \& Sons, New York, 1979).*

Reed, R. P. and A. F. Clark, Materials at Low Temperatures (American 
Society for Metals, Metals Park, OH, 1983).*

Reichner, P., "Pressure-Wear Theory for Sliding Electrical Contacts," IEEE Transactions on Components, Hybrids, and Manufacturing Technology, 4(1), 45-51 (1981).

Reif, F., Fundamentals of Statistical and Thermal Physics, (McGraw-Hill, New York, 1965)

Ripin, B. H. et al., "Laser-Plasma Interaction and Ablative Acceleration of Thin Foils at $10^{12}-10^{15} \mathrm{~W} / \mathrm{cm}^{2}$," Physics of Fluids, 23(5), 1012-1030 (1980).

Roberts, E. W., "Thin Solid Lubricant Films in Space," Tribology International, 23(2), 95-104 (1990).

Rose, D. J. and M. Clark, Jr., Plasmas and Controlled Fusion (MIT Press and John Wiley and Sons, New York, 1961).

Saka, S. et al., "Development of Hydrogen Pellet Acceleration System Using a Two-Stage Light Gas Gun" (Kobe Steel, Ltd. Nishi-ku, Kobe, 651-22 Japan, 1991).

Salman, W. A., and H. Scammell eds., Proc. Symposium on Lubrication in Space, Baird Atomic, Inc. and Arthur D. Little, Inc., Cambridge, MA, NTIS N64-19952 (1963).

Sarkar, A. D., Wear of Metals, (Pergamon Press, New York, 1976).*

Schneider, J. M., and C. D. Hendricks, "Source of Uniform Size Liquid

Droplets," Review of Scientific Instruments, 35(10), 1349-1350 (1964).

Schramm, R. E., A. F. Clark, and R. P. Reed, A Compilation and Evaluation of Mechanical, Thermal, and Electrical Properties of Selected Polymers, NBS Monograph 132 (National Bureau of Standards, Boulder, Colorado, 1973).*

Seigel, A. E., "The Theory of High Speed Guns," AGARDograph91 (1965). Shapiro, A. B., TOPAZ2D -- A Two-Dimensional Finite Element Code for 
Heat Transfer Analysis, Electrostatic, and Magnetostatic Problems,

Lawrence Livermore National Laboratory, Livermore, CA, UCID-20824, (1986).

Shapiro, A. H., The Dynamics and Thermodynamics of Compressible Fluid Flow (Ronald Press, New York, 1953).*

Smythe, W. R., Static and Dynamic Electricity, 3rd ed. (McGraw Hill, New York, 1968).*

Souers, P. C., Hydrogen Properties for Fusion Energy (University of California Press, Berkeley, CA, 1986).*

Stupp, B. C., "Synergistic Effects of Metals Co-Sputtered with $\mathrm{MoS}_{2}$, "Thin Solid Films, 84, 257-266 (1981).

Taylor, C. F., The Internal Combustion Engine in Theory and Practice, 2nd ed.

(M. I. T. Press, Massachusetts Institute of Technology, Boston, Massachusetts, 1979).*

Timoshenko, S. and S. Woinowski-Krieger, Theory of Plates and Shells, 2nd ed. (McGraw Hill, New York, 1959).*

VanSant, J. H., Conduction Heat Transfer Solutions, Lawrence Livermore

National Laboratory, Livermore, CA, UCRL-52863 Rev. 1 (1983).*

Wiczer, J. J. et al., "AlGaAs/ GaAs Radiation Hardened Photodiodes,"

Proceedings of SPIE, Volume 506, Fiber Optics in Adverse Environments II (1984).*

Waganer, L. M. et al., Inertial Fusion Energy Reactor Design Studies, McDonnell Douglas Aerospace, Saint Louis, MO, DOE/ER-54101 (1992).*

Weast, R. C. ed., Handbook of Chemistry and Physics, 52nd ed. (The Chemical Rubber Co., Cleveland, $\mathrm{OH}, 1971)$ * $^{*}$ 
Whirley, R. G., and B. E. Englemann, DYNA2D A Nonlinear, Explicit, TwoDimensional Finite Element Code for Solid Mechanics User Manual, Lawrence Livermore National Laboratory, Livermore, CA, UCRL-MA110630 (1992).

Wuerker, R. F., H. Shelton, and R. V. Langmuir, "Electrodynamic

Containment of Charged Particles," Journal of Applied Physics, 30, 342 (1959).*

Yang, W. H., and W. W. Feng, "On Axisymmetrical Deformations of Nonlinear Membranes," Transactions of the ASME, Series E, Journal of Applied Mechanics, 37, 1002 (1970).*

Zabar, Z. et al., "Test Results for Three Prototype Models of a Linear Induction Launcher," IEEE Transactions on Magnetics, 27, 558-562 (1991).

Zuckerman, D. S. et al., "An Induction Linac Driven Heavy-Ion Fusion Systems Model," Fusion Technology, 13(2), 217-254 (1988).* 